\title{
Arabidopsis basic leucine Zipper transcription factors function as quantitative modulators of auxin mediated transcription
}

\author{
Dissertation \\ zur Erlangung des Doktorgrades \\ „Doctor rerum naturalium“ \\ der Mathematisch-Naturwissenschaftlichen Fakultäten \\ der Georg-August-Universität zu Göttingen
}

\author{
vorgelegt von \\ Christoph Weiste \\ aus Koblenz
}

Göttingen 2011 
Mitglieder des Betreuungsausschusses: Prof. Dr. Wolfgang Dröge-Laser Prof. Dr. Christiane Gatz

Referent:

Prof. Dr. Wolfgang Dröge-Laser

Korreferentin:

Prof. Dr. Christiane Gatz

Tag der mündlichen Prüfung:

26.04.2011 



\section{Table of Contents}

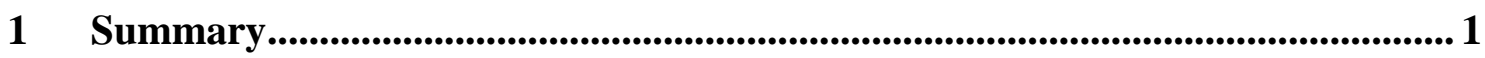

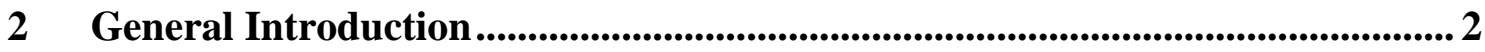

2.1 Phytohormones control diverse plant developmental and environmental

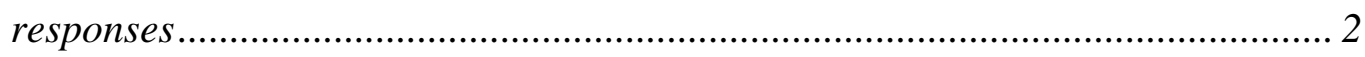

2.2 Auxin is an essential plant hormone that controls a wide range of developmental and growth-related processes in the course of plant life.............. 3

2.3 Auxin metabolism and homeostasis is controlled by multiple coordinated

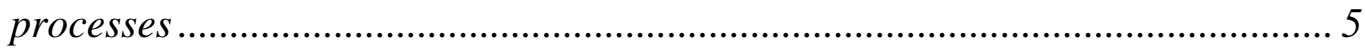

2.4 Differential auxin distribution mediated by directional auxin transport initiates and controls auxin related developmental and environmental processes

2.5 Auxin gradients are primarily converted to local differentiation events via auxin mediated transcription

2.6 Auxin responsive genes are presumably regulated by antagonistic histone acetylation and deacetylation

2.7 Members of the bZIP-and MYB-TF families have been implicated in modulating auxin mediated responses

2.8 The C/S1 network of AtbZIP-TFs is involved in the plant's energy homeostasis and has been found to modulate plant growth responses

2.9 Outline and objectives of the thesis

3 Results

3.1 Chapter 1: Bioinformatic cis-element analysis performed in Arabidopsis and rice reveals bZIP- and MYB-related binding sites as coupling elements in AuxRE mediated auxin responsive transcription....

3.2 Chapter 2: G-box related cis-elements and their cognate bZIP transcription factors function as quantitative modulators of auxin-mediated growth responses.

3.3 Chapter 3: Arabidopsis AtbZIP11-related transcription factors modulate auxinmediated transcription by recruiting the histone acetylation machinery....

3.4 Chapter 4: Heterodimers of the Arabidopsis transcription factors bZIP1 and bZIP53 reprogram amino acid metabolism during low energy stress...... 138 
4.1 The G-box related element (GRE) and its cognate bZIP transcription factors constitute a novel regulatory unit which quantitatively modulates auxinregulated transcription

4.2 AtbZIP11 related transcription factors interfere with the AUX/IAA - GH3 feed-back mechanism which modulates auxin mediated growth responses .... 171

4.3 AtbZIP11-related transcriptional activators modulate auxin-mediated gene expression by recruiting the histone acetylation machinery...

4.4 BZIP factors of the C/S1 network are proposed to balance plant growth responses and adaptive metabolic reprogramming under energy deprived conditions.

5 Appendix. 185

5.1 Abbreviations 185

6 References. 


\section{Summary}

The essential plant growth hormone auxin orchestrates a wide range of developmental and environmental processes in the course of plant life. In general, these responses are predominately implemented by the encoded activity of auxin responsive genes, which are corporately regulated by the family of Auxin Response Factors (ARFs) and the class of AUX/IAA proteins. Whereas ARF transcription factors (TFs) exert their transactivating properties upon direct binding to their cognate Auxin Response Elements (AuxREs), the AUX/IAA transcriptional repressors contact the ARF proteins to modulate their activity. In order to sustain optimal plant growth, auxin mediated responses have to be adjusted according to the prevailing endogenous and environmental conditions. Thus an integration of the corresponding stimuli into auxinrelated transcriptional patterns is required. Bioinformatic promoter cis-element analyses revealed that promoters of auxin responsive genes are not only significantly enriched for AuxREs, but also for the G-BOX RELATED ELEMENTS (GREs) and MYB RESPONSIVE ELEMENTS (MREs). Using the Arabidopsis AtGH3.3 promoter as an auxin responsive model system, a combinatorial control of auxin-mediated transcription by a complex arrangement of these, in part redundantly acting, cis-elements has been demonstrated. Whereas AuxREs function as auxin-dependent switches, GREs and MREs act as quantitative modulators. Applying a trans-activation screening approach in protoplasts, members of the C/S1 network of basic leucine zipper (bZIP) TFs have been identified, which enhance and sensitize auxin-mediated transcription via binding the GRE cis-element. Complementary, gain- and loss-of-function approaches in transgenic plants confirm that the closely related group S1 AtbZIP2, -11 and -44 TFs modulate auxin-induced transcription and are capable to alter typical auxin-related growthresponses, such as primary root growth, lateral root formation, root hair density and gravitropism. Histochemical expression analysis of the auxin responsive DR5:GFP reporter suggests bZIP-dependent alterations in auxin distribution and/or signalling. Ensuing studies on the mechanistical action of the group S1 bZIPs on auxin mediated transcription revealed that particularly AtbZIP11-related TFs are able to recruit the 
SAGA-like acetylation machinery via their $\mathrm{N}$-terminal activation domain. Pharmacological and reverse genetic approaches clearly define the impact of histone acetylation in auxin-induced transcription. In fact, Chromatin-Immunoprecipitation (ChIP) analyses confirm bZIP-dependent recruitment of the histone acetylation machinery and RNA-Polymerase II. Altogether, these data suggest a novel bZIPmediated mechanism to fine-tune chromatin accessibility during auxin-induced gene activation. As C/S1 bZIP-TFs are reprogramming the primary metabolism in response to energy stress, the GRE/bZIP module might function as a "rheostat" which provides means to balance auxin-mediated growth responses on the energy status of the plant.

\section{General Introduction}

\subsection{Phytohormones control diverse plant developmental and environmental responses}

Plant hormones, also designated as phytohormones are essential molecules that regulate many aspects of plant metabolism to ensure coordinated plant growth, development, reproduction and/or plant defence (Wang and Irving, 2011; Pieterse et al., 2009). By definition, they are signalling molecules that are released by specific cells or tissues and often affect the metabolism of distant responsive cells or tissues, already at low amounts (Taiz and Zeiger, 2006). The responsiveness of the corresponding recipient cells and tissues varies and is dependent on their individual sensitivity to the hormone, which in turn can be affected by the tissues current developmental stage and the prevailing environmental conditions (Vanneste and Friml, 2009). This enables the plant to address adaptive, metabolic reprogramming in selected tissues, by locally altering the hormone concentration or the hormone sensitivity of the respective receiver cells.

Classically five types of phytohormones are known, which fit the prevailing definition of hormones. These are abscisic acid (ABA), auxin, cytokinins, ethylene and gibberellins (Wang and Irving, 2011). Besides these, further signalling molecules, such as brassinosteroids, jasmonates, salicylic acid (SA), systemin and strigolactones have 
been described to fulfil phytohormone functions (Pieterse et al., 2009). New insights into hormone action and the extensive crosstalk between them, led to a detailed picture of how hormones regulate diverse plant processes (reviewed in Davies, 2004). The essential plant hormone auxin is one of the best-characterised hormones as it is involved in several plant developmental and growth-related responses. In the following section an overview of auxin action and homeostasis is presented.

\subsection{Auxin is an essential plant hormone that controls a wide range of developmental and growth-related processes in the course of plant life}

The long lasting history of plant biology is clearly intertwined with the conception and discovery of the plant hormone auxin. In numerous decades of plant science the contribution of diverse, newly emerging disciplines such as molecular-, structural- and cellular biology helped to unravel many, but certainly not all, of the hormones' secrets leading to a comprehensive picture of auxin action in plant development and growth (Abel and Theologis, 2010). The structural nature of the growth promoting substance auxin, named after the greek verb auxano, which means "to grow or expand", was first discovered in 1936 and was found to be a small and simple structured molecule, comprised of a planar hydrophobic indole ring system linked to acetic acid (Went et al., 1937). Soon after its identification it became apparent that Indole-3-Acetic $\underline{\text { Acid }}$ (IAA) is the principal auxin in all land plant species (Haagen-Smit et al., 1946; Rensing et al., 2008; De Smet et al., 2011; Abel and Theologis, 2010). During their life cycle the hormone controls a diversity of processes. The best-characterised auxin-mediated responses (Figure 1) are the enhancement of cambial activity and cell division (Snow et al., 1935), cell elongation which is mediated by the auxin-associated cell wall relaxation (Hoson et al., 1991) and cell differentiation of parenchyma cells to xylem vessels or phloem cells during the formation of vascular tissue (Thimann et al., 1977). On more systemic level, several developmental processes are controlled by auxin, such as the determination of embryo polarity (Möller and Weijers, 2009), the shoot (apical dominance and shoot elongation) (Vernoux et al., 2010) and root meristem outgrowth (primary root elongation, initiation of lateral and adventitious roots and root hairs) (Pitts et al. 1998; Rahman et al. 2002; Ishida et al. 2008; Peret et al. 2009), the initiation and 
formation of female flowers (Nole-Wilson et al., 2010) and the inhibition of leaf and fruit abscission (Shoji et al., 1951; Ellis et al., 2005). In addition, environmental stimuli, like gravity (gravitropism) or light (phototropism) are integrated into directed plant growth by the plant hormone (Muday, 2001). In most tissues auxin responses are concentration dependent and different tissues respond in a distinct manner to varying amounts of auxin (Thimann, 1938; Ludwig-Müller, 2011). While adequate concentrations of the hormone can stimulate specific responses, higher levels can act inhibitory (Thimann, 1938). This fact is also utilized in the agronomic field to control undesired plant growth by applying synthetic, highly stable auxin analogs which operate as herbicides (Grossmann, 2007).

Besides its role in plant development and growth, auxin has been reported to be involved in the establishment of plant - pathogen interactions. It was postulated to act in an antagonistic crosstalk with the signalling molecule salicylic acid (SA), which is known to be a crucial key promoter of plant pathogen defence responses (Pieterse et al., 2009). Indeed, specific auxin mediated responses are suppressed after pathogen attack and/or SA accumulation (Wang et al., 2007; Park et al., 2007), which presumably can be ascribed to a plants' derived mechanism to impair auxin signalling (Navarro et al., 2006). In contrast to this, various pathogens have evolved strategies to bypass this adaptation of auxin signalling by actively producing large amount of auxins (Glickmann et al., 1998) or titrating repressors of auxin mediated expression (Padmanabhan et al., 2008). These findings indicate that auxin can act as a susceptibility factor to enhance pathogen virulence. 


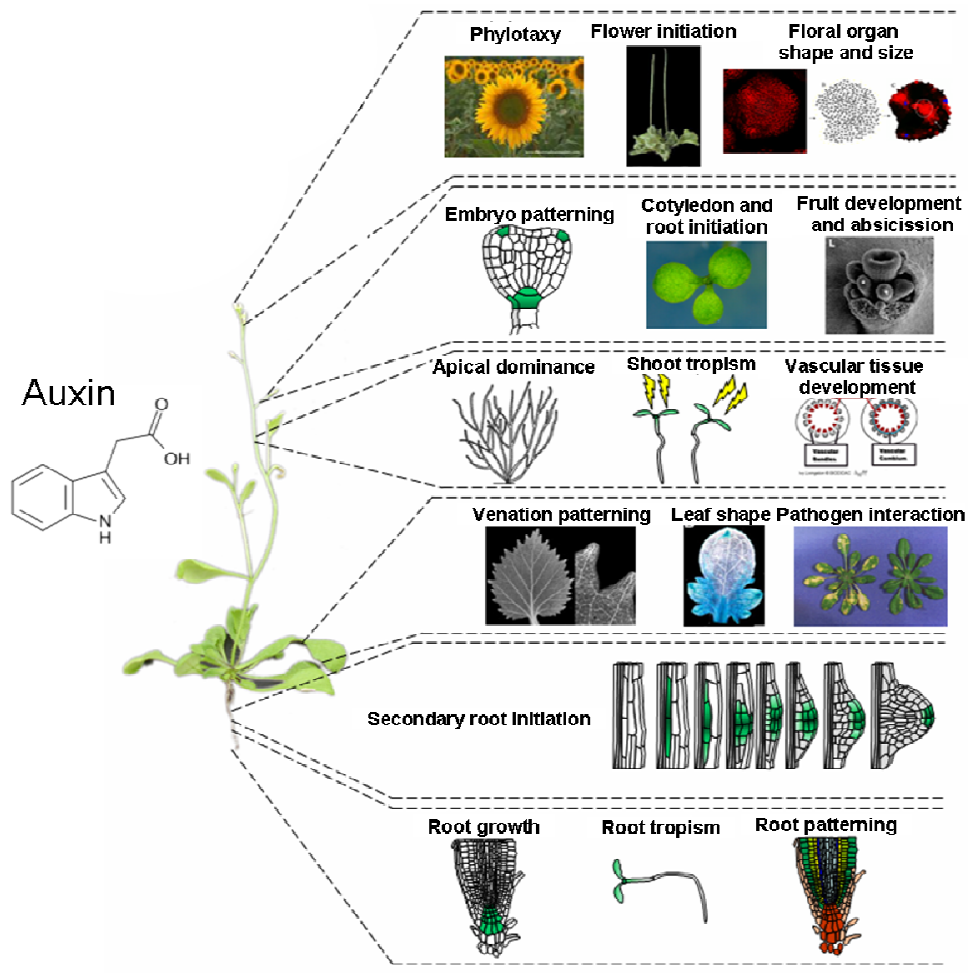

Figure 1: Overview of typical auxin mediated plant responses (adapted from the Thierry Gaude lab homepage at http://www.ens-lyon.fr/RDP/SiCE/english/index-uk.html).

In conclusion, these multi-facetted functions of auxin visualise that the endogenous auxin levels and auxin-mediated plant responses have to be strictly and corporately regulated, to ensure proper plant growth, development and survival.

\subsection{Auxin metabolism and homeostasis is controlled by multiple coordinated processes}

The regulation of endogenous auxin concentrations and auxin-mediated responses is thought to be predominantly executed by a trinity of co-ordinated processes, including auxin metabolism, auxin distribution and the individual, cellular auxin response (reviewed in Abel and Theologis, 2010). Auxin metabolism involves both, the spatiotemporal regulation of auxin biosynthesis and degradation on the one hand and the modulation of the existing, bioactive pool of free auxin by reversible or irreversible conjugation to high or low molecular weight molecules, on the other hand (Chandler, 2009). Until today several auxin and auxin precursor (e.g IPA) conjugates with low 
molecular weight moieties such as amino acids, sugars, myo-inositol or methyl groups and high molecular weight moieties such as peptides and proteins have been described (reviewed in Bajguz and Piotrowska, 2009; Chandler, 2009). As only free auxin is established to be the biological active substance, temporary or constant hormone conjugation is postulated to adjust auxin homeostasis by partitioning the hormone to storage compounds or commit them to degradation (Woodward et al., 2005). In this respect, only the relevance and occurrence of the auxin - amino acid conjugates has been studied in greater detail and in diverse plant species. In accordance to this, repressively acting auxin conjugation to diverse amino acids is catalysed by the encoded activity of the early auxin responsive GH3 genes (Staswick et al., 2005). Based on their rapid auxin triggered expression and catalytic activity they constitute a negative feedback mechanism to adjust auxin homeostasis. Homologs of these genes, which encode for IAA-amido synthetases, are classified as group II GH3s and are found in all higher land plants (Staswick et al., 2005; Ludwig-Müller, 2011). Depending on the specificity of the corresponding enzyme, a broad range of amino acids can serve as substrates. Thus far, GH3 formed conjugates with aspartate (Asp), glutamate (Glu), phenylalanine (Phe), alanine (Ala), leucine (Leu), glycine, isoleucine, methionine, proline, tyrosine, glutamine, valine and tryptophan (Trp) have been identified (Staswick et al., 2005). As IAA-Ala, IAA-Leu and IAA-Phe conjugates are described to be substrates of the, so far characterised IAA-amido hdyrolases, which rapidly convert the conjugates to their initial molecules, they apparently serve as temporary, inactive IAA storage reserves (Davies et al., 1999; Rampey et al., 2004; Savic et al., 2009). In contrast to this, IAA conjugation to Asp and Glu was found to be irreversible and leads to oxidative catabolism of IAA (Östin et al., 1998; Yee Tam et al., 2000). Since it has been presumed that conjugated IAA is principally biological inactive, it was remarkable that auxin conjugation to Trp reversed the hormone's activity, now suppressing some auxin-related responses (Staswick, 2009).

Besides this rapid modulation of auxin homeostasis by regulating the existing auxin pool via repressive conjugation, the hormone concentration can also be adjusted by controlled spatio-temporal auxin biosynthesis. Although many enzymatic steps of the proposed auxin biosynthetic pathways are yet undisclosed and the contribution of each of them is, due to the extensive functional redundancy between them, difficult to assess, 
an outline of the most discussed IAA biosynthetic pathways can be made (Figure 2). To date one Trp-independent and four Trp-dependent routes for IAA biosynthesis have been postulated, each of them cytoplasmically localised and designated to an intermediate that is a hallmark of it (reviewed in Vanneste and Friml, 2009). These are, with respect to the Trp-dependent routes, the indole-3-acetamide (IAM) pathway, the indole-3-acetaldoxime (IAOx) pathway, the tryptamine (TAM) pathway and the indole3-pyruvic acid (IPA) pathway (reviewed in Woodward and Bartel, 2005; Chandler et al., 2009) and related to the Trp-independent route, the indole-3-glycerol phosphate (IGP) pathway (Ouyang et al., 2000).

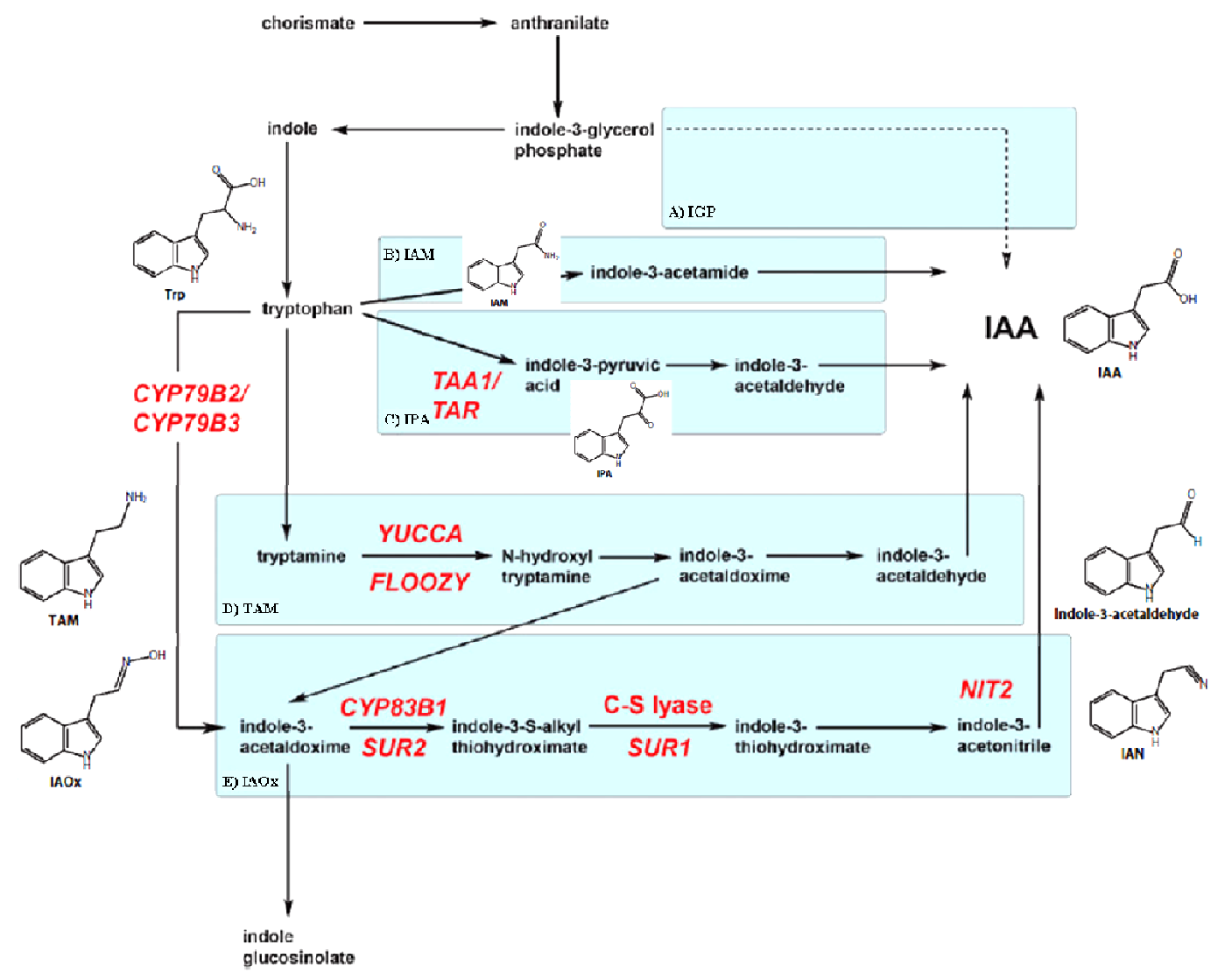

Figure 2: Overview of auxin biosynthesis pathways. A) Postulated Tryptophan (Trp) independent biosynthetic pathway, IGP. B - E) Trp-dependent biosynthetic pathways such as the B) IAM- C) IPA- D) TAM- and E) IAOx-pathway. Figure was adapted from Chandler et al., 2009 and complemented with figures of IAA intermediates from Zhao, 2010.

Thus far, only the TAM and IPA biosynthetic pathways have been highlighted to notably contribute to auxin dependent developmental processes in planta (Vanneste and 
Friml, 2009). Referring to the TAM pathway the initial rate-limiting step is catalysed by flavin monooxygenase-like enzymes of the YUCCA family (Zhao et al., 2001). They convert the Trp derivative TAM to N-hydroxyltryptamine, a precursor of IAOx that can be subsequently used in the biosynthesis of IAA (Zhao et al., 2001). Applying reverse genetic approaches it could be demonstrated that mutations in multiple, co-expressed YUCCA genes lead to local auxin imbalances to an extend that it resulted in auxinrelated developmental defects such as abnormal leaf venation, root pole specification and floral organ patterning (Cheng et al., 2006; Cheng et al., 2007), indicating that the YUCCA-mediated TAM pathway is at least one of the major IAA biosynthetic routes in Arabidopsis.

The relevance of the IPA pathway in IAA biosynthesis has only recently been shown with the identification of a gene family encoding Arabidopsis Trp aminotransferases (TAAs). They catalyse the transamination of Trp to IPA, which afterwards can be further decarboxylated to IAA. Mutants in TAA genes show reduced free IAA levels suggesting that also the TAA-dependent IPA pathway significantly contributes to total IAA production (Stepanova et al., 2008; Tao et al., 2008). Accordingly, the TAA mutants show an attenuated differential auxin distribution that coincides with pronounced auxin-related defects in gravitropism, embryogenesis and vascular tissue differentiation (Stepanova et al., 2008; Vanneste and Friml, 2009). Due to the fact that mutants in the key enzymes of the TAM and IPA pathways exhibit in part similar auxinrelated phenotypes and likely share at least a few enzymatic steps, it has to be shown whether these pathways indeed represent independent or at least partially redundant routes for IAA production (Vanneste and Friml, 2009). In terms of regulation of these auxin biosynthesis pathways, only rudimentary knowledge has been accumulated. Nevertheless, it becomes apparent that specific environmental signals such as light quality (Tao et al., 2008) or developmental signals which are integrated by the plant hormone ethylene (Stepanova et al., 2008) can regulate TAA transcription and therefore TAA-dependent IAA biosynthesis. This exemplarily demonstrates that auxin biosynthesis pathways are likely co-ordinately regulated to provide auxin during plant development and in response to environmental cues and thereby integrate internal and external stimuli in auxin mediated responses. 
Auxin biosynthesis is constantly and primarily implemented in the meristems of the shoot apex (Vernoux et al., 2010), but also to a minor degree in roots (Ljung et al., 2005; Ikeda et al., 2009; Petersson et al., 2009) and temporarily also in the actively growing tissues like the developing embryo (Möller and Weijers, 2009), young leaves (Ljung et al., 2001) and fruits (Epstein et al., 2002). In order to enable proper auxin mediated responses in these and more source distant plant tissues, which completely or partially depend on auxin import, the hormone has to be directionally translocated from the main sources to the corresponding sink tissues (Vernoux, 2010).

\subsection{Differential auxin distribution mediated by directional auxin transport initiates and controls auxin related developmental and environmental processes}

The understanding of auxin action was substantially shaped by the finding that auxin is differentially distributed throughout the plant and its tissues and that this feature is universally associated with the correct manifestation of diverse auxin related responses (reviewed in Tanaka et al., 2006). For instance the auxin mediated tropic responses are established by the formation of local auxin maxima and minima, also referred to as auxin gradients. As mentioned earlier, varying tissues can respond differently to distinct auxin concentrations. With respect to auxin mediated cell elongation, a local auxin accumulation in root cells inhibits their elongation, whereas shoot cells respond with an enhanced elongation (Thimann, 1938; Tanaka et al., 2006). Based on this principle, roots and shoots redirect their growth during tropic responses in opposite ways (Vanneste and Friml, 2009). For example gravity stimulation leads to a differential auxin distribution across the root and stem organs which induces differential organ growth and therefore organ bending. Vividly this means that whenever a change in gravity stimulus occurs auxin accumulates at the lower site of the root- and shoot organs, which locally inhibits cell elongation in the lower root parts, which therefore bend downwards and locally stimulate cell growth within the lower shoot parts, which then bend upwards (Tanaka et al., 2006). A similar mechanism of stimulus induced differential auxin distribution and therefore directional growth is the plants' phototropic response. To enable optimal plant growth, the plant needs to grow towards the light to ensure saturated photosynthetic activity. The requisite plant growth adjustment is 
mediated by a local auxin accumulation at the more shaded site of the shoot, leading to locally enhanced auxin mediated cell elongation and thereby to light directed growth (reviewed in Whippo and Hangarter, 2006). Besides the role of auxin gradients in regulating growth in response to environmental stimuli, it also has multiple functions in developmental processes such as embryogenesis and organogenesis (Vanneste and Friml, 2009). For example, in the early embryonic stages, dynamic patterns of auxin maxima are established, which initially determine the embryo polarity by specifying the apical cell and later in development assign the root pole and the locus of cotelydon formation (Friml et al., 2003). During post-embryonic plant growth auxin gradients set developmental marks of de novo leaf, flower and lateral root organogenesis (Benkova et al., 2003). In general these developmental processes are initiated by a local auxin accumulation at the site of prospective organ growth (Dubrovsky et al., 2008; Heisler et al., 2005) and are subsequently maintained with the establishment of an auxin gradient along the growth axis of the developing primordium with the auxin maximum at its tip (Benkova et al., 2003). Transferred to the initiation of primary and lateral roots, this means that once the root meristem is set and functional, a stable auxin gradient is constituted with its maximum in the quiescent center and young columella cells (Sabatini et al., 1999; Friml et al., 2002), which is required to maintain the pattern and activity of the root meristem (Blilou et al., 2005). A similar mechanism is operating in the early leaf development, in which local auxin maxima are not only essential to initiate the formation of the leaf primordia, but also control the development of internal structures like the vascular tissue during leaf venation (Mattsson et al., 2003; Scarpella et al., 2006). Notably, not only local auxin maxima but also minima control diverse developmental processes. For instance it had been demonstrated that local auxin depletion is crucial for seed dispersal, as an auxin minimum specifies the valve margin separation layer, which is the restricted opening zone of Arabidopsis siliques (Sorefan et al., 2009). Altogether these examples illustrate the importance of differential auxin allocation within the plant and plant tissues to enable coordinated plant development in response to intrinsic and external stimuli.

To establish and dynamically regulate these stimuli-induced auxin gradients, the hormone has to be directionally transported from the biosynthetic active organs to the sites of auxin action. The bulk long distance auxin transport from the main source 
tissues, such as the shoot apex to the main sink tissues, like the root is mediated by the rapid (up to $7 \mathrm{~cm} / \mathrm{h}$ ) transport pathway of the membraneless phloem sieve elements (Marchant et al., 2002; Tsurumi and Wada, 1980), which also carries carbohydrates, proteins and mRNAs but also the phytohormones $\mathrm{ABA}$ and cytokinins to their corresponding sink tissues (Robert and Friml, 2009). Besides this rapid, but rather undirected auxin bulk transport, which distributes auxin throughout the plant, auxin can much slower $(10 \mathrm{~mm} / \mathrm{h})$, but in a plant hormone unique fashion also move in a directional manner between cells and tissues (reviewed in Vanneste and Friml, 2009; Overvoorde et al., 2010). This directed short distance movement is thought to be based on both, passive diffusion and active translocation. Whereas the passive diffusion of the polar auxin molecule through unpolar membranes into the cell can be explained by the so-called chemiosmotic hypothesis (Rubery and Sheldrake, 1974; Raven et al., 1975), the directed cellular auxin in- and efflux is ascribed to energy demanding auxin transport facilitators. A combined view of both systems gives a comprehensive picture of how a diffuse auxin flow can be dynamically navigated to constitute local auxin gradients (reviewed in Vanneste and Friml, 2009). Referred to the chemiosmotic hypothesis, the apoplastic space, which surrounds all cells has, due to the activity of membrane associated $\mathrm{H}^{+}$-ATPases, a relatively low $\mathrm{pH}$ of around 5.5. In this environment a portion of the weak acid auxin (IAA ${ }^{-}$) becomes protonated (IAAH) and therefore more lipophilic, which enables the now unpolar molecule to freely diffuse through the plasma membrane into the cell. This passive auxin uptake is further actively supported by $\mathrm{H}^{+} / \mathrm{IAA}^{-}$symporters, which belong to a small gene family of AUX1/LAX influx carriers (Bennett et al., 1996; Swarup et al., 2001). Once inside the neutral cytosol ( $\mathrm{pH} \sim 7.0$ ), the auxin molecules become again deprotonated and are therefore trapped inside the cell. The only possibility for auxin to leave the intracellular space is to be actively transported through the plasma membrane by specific efflux carriers. Thus far, two types of auxin efflux carriers have been described, namely the gene family members of the PIN-formed (PIN) proteins (Petrasek et al., 2006) and the Pglycoproteins of the ATP-Binding Cassette B (ABCB) transporters (Geisler and Murphy, 2006). Based on the activity of the described in- and efflux transport machinery, the intracellular auxin concentration can be dynamically and effectively controlled. As it has been found that specific PIN efflux protein family members are 
individually expressed in distinct root tissues and exhibit a polar distribution within the cellular plasma membrane, the auxin flow can be directionally translocated among cells and within tissues (reviewed in Friml, 2010). An impressive example of concerted auxin transport, mediated by the directed auxin efflux controlled by several PIN proteins, can be observed in the root apex. At this, auxin is actively transported from the auxin maximum, which is located in the meristematic zone of the root tip, through the epidermal and cortex cells to the distal basal meristem and then back towards the root tip, by the collaborated work of AUX1/LAX mediated influx and PIN protein directed efflux. This, PIN protein conducted, self-contained auxin reflux system (Figure 3), which is also known as the inverted fountain of auxin root movement, enables coordinated root tissue development and root meristem maintenance (Blilou et al., 2005).

Figure 3: Schematic view of PIN mediated polar auxin transport in the post embryonic root apex. Auxin flow is directed by the family of PIN auxin efflux carriers, which maintain the stem cell niche by controlling cell division in the meristematic division zone (DIV) and cell elongation and -differentiation in the root`s elongation zone (EL). Figure was adapted from Blilou et al., 2005.

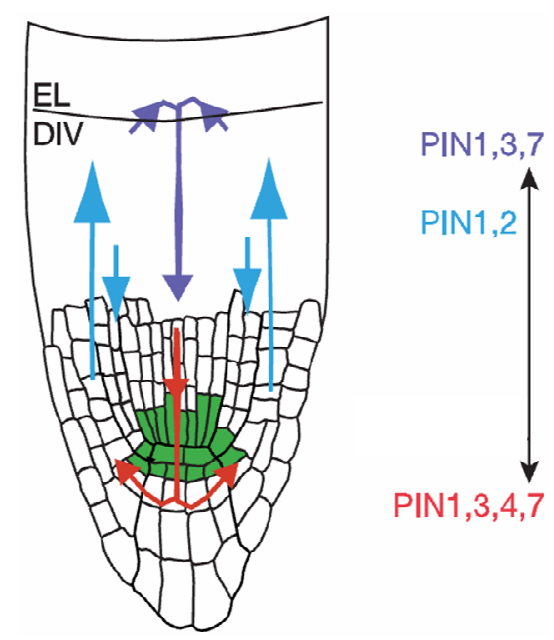

As PIN proteins are constantly internalized by a clathrin coated vesicle dependent pathway, recycled in intracellular endosomal compartments and transported back to the plasma-membrane, they can be rapidly relocated to different sites of the cell (Friml, 2010). This PIN transcytosis enables a rapid and adaptive redirection of the auxin flow to establish and manage auxin gradients in response to changing developmental and environmental conditions (Friml et al., 2002; Grunewald and Friml, 2010).

At a glance this illustrates that besides local auxin biosynthesis and auxin homeostasis, especially the directed auxin transport substantially contributes to the formation of auxin gradients and their related local auxin responses. 


\subsection{Auxin gradients are primarily converted to local differentiation events via auxin mediated transcription}

It has been demonstrated that the formation of local auxin gradients initiates and controls diverse auxin mediated responses. However the question arises how single cells and tissues are able to individually interpret this simple auxin signal and convert it into the various auxin-related developmental and environmental responses. In the recent years, tremendous progress has been made to unravel this mystery. These days it becomes more and more apparent that auxin mediated responses are predominantly executed by the encoded activity of auxin responsive genes, which are presumably regulated in a cell or tissue specific manner by a sophisticated, combinatorial transcriptional control mechanism. The most upstream element of this intracellular auxin signal transduction pathway, the auxin receptor complex, was encountered during the 1990s. In initial genetic screens for partially or completely auxin insensitive mutants several components of the protein ubiquitination machinery were identified, such as

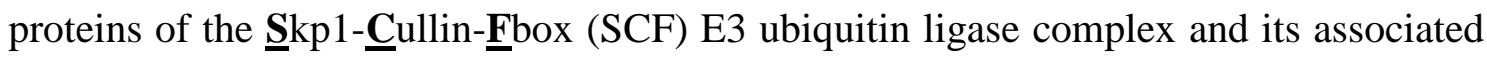
auxin binding F-box protein, Transport Inhibitor Response 1 (TIR1) (Leyser et al., 1993; Ruegger et al., 1998; Pozo et al., 1998; Tan et al., 2007). In particular for the crucial TIR1 auxin receptor component, three additional closely related Auxin signalling $\underline{\mathbf{F}}-\underline{B}$ ox proteins (AFB1-3) were found, which are also able to bind auxin and to trigger auxin related responses via the SCF complex. However, based on the observations that 1.) single mutations in TIR1 and specific AFBs already lead to various auxin insensitive phenotypes, 2.) their individual expression is in part differentially regulated and 3.) they belong to distinct clades which are evolutionary conserved among all land plants, it can be assumed that they are only partially functional redundant and likely also implement defined auxin responses in tissue or development specific contexts (Parry et al., 2009; Rensing et al., 2008).

SCF ubiquitin ligase complexes are known to specifically polyubiquitinate target proteins, which are thereby labelled for degradation by the $26 \mathrm{~S}$ proteasome. This anticipated that regulation of protein stability might be an important part of the auxin signalling pathway. Indeed, it could recently been demonstrated that an auxin dependent degradation of specific repressors of auxin mediated transcription, referred to as 
AUX/IAA repressors, is mediated by the SCF- ${ }^{\text {TIR1/AFB }}$ complex (Dharmasiri et al., 2005; Tan et al., 2007). In this process the auxin receptor component TIR1 or AFB initially binds auxin at physiological relevant concentrations in a polar cavity of the protein. Within this binding pocket, the planar unsaturated ring system of auxin or an auxin analog is positioned and stabilized via salt bridges between the auxins' carboxyl group and the receptors' internal inositol hexakisphosphate $\left(\mathrm{IP}_{6}\right)$ cofactor. By this means the unpolar ring system covers up the polar bottom of the TIR1 or AFB auxin binding pocket and forms a continuous, hydrophobic interaction surface, which now enables binding of a GWPPV amino acid core motif within the transcriptional AUX/IAA repressors (reviewed in Abel and Theologis, 2010; Dharmasiri et al., 2005; Kepinski and Leyser, 2005; Tan et al., 2007).

Downstream of the auxin perception by the SCF- ${ }^{\mathrm{TIR} 1 / \mathrm{AFB}}$ receptor complex, an interplay between two plant specific classes of transcriptional regulators constitutes the pivotal regulation unit of auxin mediated transcription. These are on the one hand the already mentioned AUX/IAA repressor proteins and on the other hand the class of $\underline{\text { Auxin }}$ $\underline{\text { Response }}$ Factors (ARFs), which can either activate or repress transcription (reviewed in Vanneste and Friml, 2009).

Members of the $A U X / I A A$ gene family were originally identified in a screen for genes that exhibit a rapid induction kinetic after exogenous auxin application (Theologis et al., 1985; Walker and Key 1982), however several representatives rather show a moderate to slow (Abel et al., 1995; Tatematsu et al., 2004) or even no auxin triggered induction (Rogg et al., 2001; Tian et al., 2002). In addition to the varying induction kinetics, several $A U X / I A A$ genes are differentially expressed in distinct parts of the plant, which suggests that specific family members might have redundant and others individual functions in tissue- or developmental-specific auxin mediated responses (Weijers et al., 2005; Tatematsu et al., 2004). In the Arabidopsis genome 29 genes encode for putative AUX/IAAs (Liscum and Reed, 2002; Remington et al., 2004). Most of them contain four highly conserved domains designated I to IV, which are separated by short variable regions (Abel et al., 1994, 1995; Reed et al., 2001). Each domain contributes to the functional properties of the AUX/IAA repressors (Overvoorde et al., 2005). The Nterminally located first domain (I) mediates the repressive activity of the protein, as it contains an ERF-associated amphiphilic repression (EAR) domain (Tiwari et al., 2004) 
which is required for the recruitment of the Groucho/Tup type transcriptional corepressor TOPLESS (TPL), that in turn is thought to mediate the interaction with histone deactetylases (HDACs) (Szemenyei et al., 2008; Liu and Karmarkar, 2008). The ensuing second domain (II) is necessary for the interaction with the TIR1 receptor and therefore confers auxin dependent proteolysis to the protein (Gray et al., 2001; Worley et al., 2000; Ouellet et al., 2001). Indeed, specific amino acid substitutions in the conserved GWPPV core motif of domain II, render the AUX/IAA repressor proteins insensitive to auxin mediated degradation and therefore strongly increase the half-lives of these proteins (Dreher et al., 2006). The last two conserved domains (III and IV) of the AUX/IAA proteins are again essential to confer transcriptional repression, as they not only serve for homo- and heterodimerisation with other AUX/IAA gene family members but also for heterodimerisation with ARF transcription factors (TFs). Based on the latter interaction the AUX/IAA proteins, which do not exhibit any DNA-binding properties on their own, are able to repress the activity of the promoter-associated ARF transcriptional activators (Kim et al., 1997; Hardtke et al., 2004; Ulamsov et al., 1997; Tiwari et al., 2003).

In contrast to the $A U X / I A A$ gene family, most of the $A R F-T F$ genes are largely constitutively expressed and encode for both, transcriptional activators and repressors (Ulmasov et al., 1999). In Arabidopsis 23 genes encode for putative ARF-TF, which are in general composed of a N-terminal B3-like DNA binding domain, a variable middle domain, which determines the activating or repressing activity of the protein and the $\mathrm{C}$ terminally located domains III and IV, which are similar to those present in AUX/IAA proteins (Kim et al., 1997; Ulmasov et al., 1997; Guilfoyle and Hagen, 2007; Remington et al., 2004; Okushima et al., 2005). ARF-TFs execute their expression

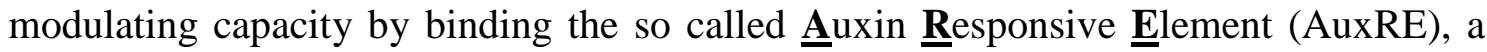
minimal consensus cis-sequence, which is necessary and sufficient to confer auxin responsiveness and which can be found in the majority of promoters from auxin inducible genes (Ulmasov et al., 1995, 1997). As already mentioned ARF-TFs, similar to the AUX/IAAs, also contain the conserved dimerisation domains (III and IV), which enable both the homo- and heterodimerisation with further ARF-TFs but also with AUX/IAA proteins (Ulmasov et al., 1997; Tiwari et al., 2003; Abel et al., 1995). By this means the ARF-TFs can either potentiate the transcriptional response, in case of specific 
ARF dimerisations, but also realise the transcriptional repression of genes in case of AUX/IAA binding (Abel and Theologis, 2010). In overview (Figure 4) the previously described SCF- ${ }^{\text {TIR } 1 / \mathrm{AFB}}$ receptor complex, the AUX/IAA proteins and the ARF-TFs are the core auxin signalling pathway components, which constitute a simple but effective regulatory circuit to control auxin responsive gene expression: Upon initial auxin perception by the SCF- ${ }^{\mathrm{TIR} 1 / \mathrm{AFB}}$ receptor complex, the repressive AUX/IAA proteins are bound by TIR1/AFBs, become polyubiquitinated by the E3 ligase subunit of the SCF complex and are subsequently degraded by the $26 \mathrm{~S}$ proteasome. This leads to a derepression of the DNA-associated ARF-TFs which presumably dimerise with other ARF-TFs to promote the expression of their auxin responsive target genes. As the expression of the AUX/IAA proteins are, in part, them self induced by auxin, they represent a primed negative feedback loop, which resets the system to the initial repressed state once the auxin concentration declines (Santner et al., 2009).

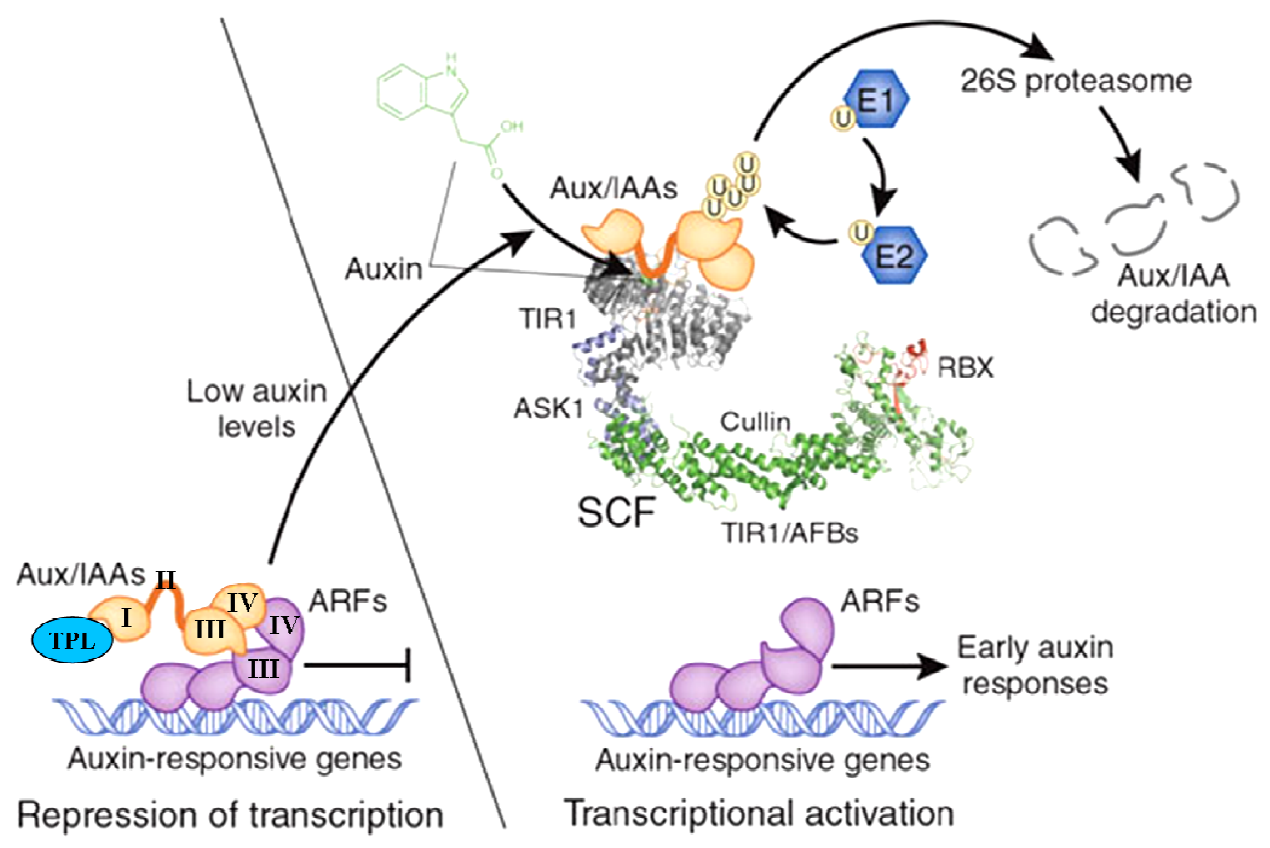

Figure 4: Model describing the auxin mediated transcriptional regulation via ARF and AUX/IAA TFs (adapted from Santner et al., 2009)

This simple model does not take into account the intrinsic complexity of the potential combinations of putative ARF and AUX/IAA homo- and heterodimers, which may differentially regulate auxin mediated gene expression in a cell- and tissue-specific 
manner or in diverse developmental contexts (Abel et al., 1994; Kim et al., 1997; Kepinski and Leyser, 2002, Guilfoyle and Hagen, 2007). Thus far the function and relevance of specific ARF-ARF and ARF-AUX/IAA pairings is yet still largely undefined. Nevertheless recent publications indicate that specific sets of co-expressed ARF and AUX/IAA proteins might indeed corporately regulate mutual sets of auxin responsive target genes and auxin mediated responses (Weijers et al., 2005; Weijers and Jürgens, 2004; Muto et al., 2007).

While the vast majority of auxin mediated responses, in particular the developmental and growth-related responses, can certainly be attributed to the SCF- ${ }^{\mathrm{TIR} 1 / \mathrm{AFB}}$ mediated transcriptional regulation of auxin responsive genes, it should be taken in consideration that some very rapid auxin triggered responses are presumably exerted by additional transcriptional independent mechanisms. Auxin for example triggers an extremely rapid (within 5 seconds) increase in cytosolic $\mathrm{Ca}^{2+}$ (Shishova and Lindberg, 2004) and a rapid induction of apoplastic proton secretion, which is accompanied by hyperpolarization of the plasma-membrane potential (Senn and Goldsmith, 1988). Moreover, a rapid but transient auxin triggered activation of a Mitogen- $\underline{A}$ ctivating Protein $\underline{\text { Kinase (MAPK) }}$ cascade has been demonstrated, which is likely involved in a negative feedback mechanism, as it suppresses the expression of specific auxin responsive genes (Mockaitis and Howell, 2000; Lee et al., 2009; Kovtun et al., 1998). Finally, also a high

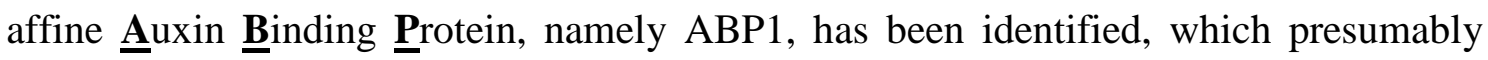
embodies an additional auxin receptor as it has been implicated in auxin mediated responses, like a rapid auxin induced hyperpolarisation of the plasma-membrane (Leblanc et al., 1999), the auxin mediated cell elongation (Chen et al., 2001; Jones et al., 1998) and also the regulation of cell cycle progression (Braun et al., 2008). However a putative downstream signalling pathway has not been identified, yet. In summary it can be assumed that the majority of auxin mediated responses is in fact exerted by the SCFTIR $1 / \mathrm{AFB}$ dependent transcriptional regulation of auxin responsive genes, whereas some rapidly auxin induced processes may rely on additional, likely transcriptional independent mechanisms (Vanneste and Friml, 2009). 


\subsection{Auxin responsive genes are presumably regulated by antagonistic histone acetylation and deacetylation}

The dynamic and reversible process of histone acetylation and deacetylation of the Nterminal tails of the nucleosomal core histones controls nucleosome positioning and chromatin condensation and therefore plays an essential role in chromatin remodelling and gene regulation (Millar and Grunstein, 2006; Kouzarides, 2007; Servet et al., 2010).

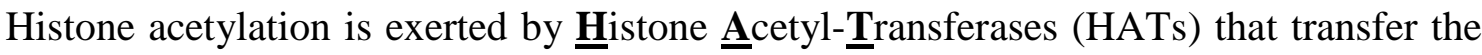
acetyl group of acetyl-CoA to specific lysine residues within the N-terminal histone domains (Servet et al., 2010). In Arabidopsis, the lysine residues of histone H3 (K9, $\mathrm{K} 14, \mathrm{~K} 18, \mathrm{~K} 23$ and $\mathrm{K} 27)$ and $\mathrm{H} 4(\mathrm{~K} 5, \mathrm{~K} 8, \mathrm{~K} 12, \mathrm{~K} 16$ and $\mathrm{K} 20)$ are found to be acetylation or deacetylation targets, respectively (Zhang et al., 2007; Earley et al., 2007). Upon acetylation the positive charge of the histone tails is neutralized, which decreases their affinity for the negatively charged DNA, thereby loosening the chromatin package, which facilitates binding of transcriptional activators to their

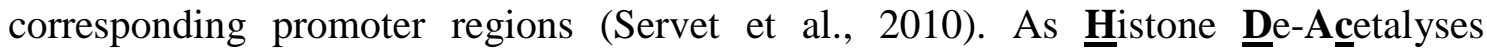
(HDACs) can reverse this process by histone deacetylation, which leads to chromatin condensation and thereby to repression of transcription, this system provides a rapid and highly dynamic switch for inter-conversion between permissive and repressive states of chromatin (Servet et al., 2010).

Quite recently a number of findings suggested that auxin responsive genes might be dynamically regulated by such a counteracting histone acetylation/deacetylation machinery. In this respect, it has been demonstrated that the repressive activity of the Arabidopsis AUX/IAA transcriptional regulator IAA12/ㅁOENLOS (IAA12/BDL) is dependent on the transcriptional co-repressor TPL, which is directly bound by the AUX/IAA protein via its EAR-domain (Szemenyei et al., 2008). A mutation in TPL leads to abnormalities in auxin mediated pre-embryonic apical-basal pole specification (Szemenyei et al., 2008). As a recessive second site mutation in the Arabidopsis histone deacetylase 19 (HDA19), intensifies the tpl mutant phenotype and one in the HAT AtGCN5 suppresses it, it had been assumed that HDA19 is located in the same repressive pathway as TPL and IAA12/BDL, whereas the transcriptional co-activator GCN5 is likely positioned in a TPL counteracting system (Long et al., 2006). Besides 
these genetic interactions it could additionally be shown that the adapter component AtADA2b which is associated with AtGCN5 in an Arabidopsis SAGA-like HAT complex (Vlachonasios et al., 2003) is important for accurate, auxin induced histone acetylation of auxin responsive promoters (Anzola et al., 2010). In line with this, mutants of AtGCN5 and AtADA2b display, in part similar pleiotropic defects in auxin related phenotypes, including dwarfism, loss of apical dominance, aberrant meristem function, abnormal root and leaf development, short petals and stamens and alterations in floral organ identity (Vlachonasios et al., 2003; Bertrand et al., 2003; Long et al., 2006; Kornet and Scheres, 2009; Servet et al., 2010). Besides AtGCN5 two further related HATs (AtHAG4 and AtHAG5), which belong to the GNAT/MYST superfamily of Arabidopsis HATs (Pandey et al., 2002) might be involved in auxin mediated processes, as it could be demonstrated that they are redundantly required for proper cell division during gametogenesis (Latrasse et al., 2008).

HATs are frequently associated in large multi-protein complexes (Servet et al., 2010). These complexes are thought to be recruited to their specific target promoters through binding of DNA-associated TFs (Servet et al., 2010). With respect to the HAT AtGCN5 and its maize homologue ZmGCN5 it has been reported that these enzymes are consulted by different TFs via an interaction with the GCN5 associated ADA2, complex adapter component (Locatelli et al., 2009). In Arabidopsis AtADA2b, for example, was found to interact with the AP2 domain containing $\underline{\mathbf{C}}$-repeat/DRE $\underline{B}$ inding $\underline{\text { Factor } 1}$ (AtCBF1) TF, which synergistically with AtADA2b and AtGCN5 promotes expression of several cold responsive genes (Stockinger et al., 2001; Mao et al., 2006). The ZmGCN5/ZmADA2 complex is recruited by the maize bZIP-TF ㅇpaque-2 (O2), which is involved in the regulation of seed storage genes, during early maize endosperm development (Bhat et al., 2004). In this respect it was recently demonstrated that $\mathrm{O} 2$ is indeed able to recruit the maize HAT complex via ZmADA2 interaction to its target promoters and that this coincides with an increased GCN5 specific H3 promoter acetylation, enhanced $\underline{\text { RNA }}$ Polymerase II (RNP II) promoter binding and increased target gene expression (Locatelli et al., 2009; Bhat et al., 2004).

Despite these findings, still very limited information about gene regulation mediated by the plant's histone acetylation/deacetylation system in general and in response to hormonal signals is available. Therefore it is yet unclear, if histone acetylation and 
deacetylation processes play a significant role in auxin mediated transcription and if so, which transcriptional regulators and/or additional molecular components might be involved in this mechanism.

\subsection{Members of the bZIP- and MYB-TF families have been implicated in modulating auxin mediated responses}

Only recently it has been reported that besides the well-established ARF- and AUX/IAA-TF families also members from stress-related TF classes are able to modulate auxin mediated transcription, which further extends the combinatorial capabilities for auxin mediated gene regulation. In this respect it has been demonstrated that for instance the R2R3 MYB-TF, AtMYB77 is able to directly bind the ARF-TF family specific C-terminus of the ARF7 protein and thereby contributes to the expression promoting capacity of the ARF7 protein (Shin et al., 2007). The relevance of this synergistic action was supported by complementary gain- and loss-of function approaches in transgenic plants. By this means it could be shown that the expression of several auxin responsive genes, which exhibited multiple $\underline{\text { MYB }} \underline{\text { Responsive Elements }}$ (MREs) in their corresponding promoters, was dependent on adequate MYB77 expression and could be enhanced by MYB77 over-expression (Shin et al., 2007). Along with this, the auxin associated lateral root formation was significantly altered in the transgenic plants (Shin et al., 2007). In vitro studies revealed that MYB77 was also capable to contact the ARF7 related transcriptional activators ARF5 and ARF6 and the transcriptional repressors ARF1 and ARF2, suggesting that MYB77 and likely further homologous R2R3 MYB-TFs might be of general importance in modulating ARF mediated auxin responsive gene expression (Shin et al., 2007). As the endogenous expression of MYB77 was found to be affected by nutrient deficiency, it has been postulated that MYB-TFs might be potential stress signal integrators to adaptively modulate auxin mediated responses (Shin et al., 2007).

Besides MYB-TFs also members of the basic leucine zipper (bZIP) TF familiy have been implicated in promoting auxin related responses. Already in the early 1990, promoter cis-element analysis of the auxin responsive soybean $G H 3$ promoter revealed that the well-characterised Auxin Responsive Elements (AuxREs) were frequently 
associated with typical bZIP-TF binding sites, such as the $\underline{\mathbf{G}}$-box $\underline{\text { related }}$ cis-elements (GREs) which had been found to contribute to the promoter's auxin-mediated induction (Liu et al., 1994; Ulmasov et al., 1995). Although direct binding of a recombinant soybean bZIP-TF has been demonstrated in vitro (Liu et al., 1997), the genuinely operating transcriptional regulators haven`t been defined, yet. Quite recently Heinekamp and co-workers (2004) identified two tobacco bZIP-TFs, namely NtBZI-1 and NtBZI-2, which can promote the expression of the soybean $G H 3$ homologous tobacco GH3 gene and bind to its associated promoter in vivo. As expression of a presumably dominant-negative acting N-terminally truncated NtBZI-1 protein furthermore resulted in diverse auxin insensitive growth phenotypes, it has been suggested that bZIP-TFs might be involved in regulating auxin mediated responses (Heinekamp et al., 2004). However, due to the limited molecular tools which are available for Nicotiana tabaccum, the functional and mechanistical characterisation of bZIP-TF action on auxin mediated transcription remained largely elusive. In the wellestablished dicot model plant Arabidopsis thaliana the closest homologs of NtBZI-1 or NtBZI-2 are classified in group $\mathrm{C}$ or group $\mathrm{S} 1$ of the Arabidopsis bZIP-TF family, respectively (Strathmann et al., 2001; Jakoby et al., 2002). Recent findings indeed indicate that these homologous proteins might also be involved in auxin related growth responses.

\subsection{The C/S1 network of AtbZIP-TFs is involved in the plant's energy homeostasis and has been found to modulate plant growth responses}

In Arabidopsis, 75 genes have been identified, which putatively encode for bZIP-TF proteins (Jakoby et al., 2002). Based on their sequence and domain homology, they were classified in 10 presumably functional groups, designated A to I and S (Jakoby et al., 2002). In general AtbZIP-TFs are characterised by a basic DNA-binding domain, which frequently harbours a nuclear localisation sequence (NLS), and an adjoining leucine zipper dimerisation domain of variable length (Jakoby et al., 2002). The leucine zipper, which builds an $\alpha$-helix, (Hurst et al., 1995) is composed of heptad repeats of leucine or similar bulky, hydrophobic amino acids such as phenylalanine, isoleucine, methionine or valine (Baxevanis and Vinson, 1993; Landschulz et al., 1988). Due to the 
domains' coiled structure these hydrophobic amino acids are unilaterally disposed, thereby constituting a hydrophobic interaction surface, which enables homo - or heterodimerisation with other bZIP proteins (Landschulz et al., 1988; O'Shea et al., 1989; Archarya et al., 2002). Although some bZIP-TFs are able to bind DNA as a monomer (Metallo and Schepartz, 1997), the majority of AtbZIPs is thought to bind DNA as homo- or heterodimer, respectively (Landschulz et al., 1988). The dimerisation of bZIP proteins is highly specific and is determined by the electrostatic attraction or repulsion of polar amino acid residues which flank the hydrophobic interaction platforms of the respective leucine zipper helices (Jakoby et al., 2002; Siberil et al., 2001; Archarya et al., 2002). Based on the high number of bZIP proteins encoded in the genome of Arabidopsis and other eukaryotic species, the bZIP's homo- and heterodimerisation properties provide an immense combinatorial flexibility to this cisregulatory system (Siberil et al., 2001; Vinson et al., 2002).

The DNA-binding domain of AtbZIP-TFs preferentially binds to promoter cis-elements with an ACGT core motif (Jakoby et al., 2002; Schindler et al., 1992; Siberil et al., 2001). However, the respective binding efficiency is dependent on additional flanking nucleotides surrounding the core sequence (Foster et al., 1994; Izawa et al., 1993). In general high affine bZIP binding sequences constitute palindromic motifs, such as the so-called A-Box (TACGTA), C-Box (GACGTC) or G-Box (CACGTG) (Izawa et al., 1993). Nevertheless, efficient bZIP binding to non-palindromic ACGT core motifs such as the ABA responsive ABRE cis-elements or the as-1 element in SA inducible promoters has also been demonstrated (Choi et al., 2000; Fukazawa et al., 2000; Schindler et al., 1992). Besides the ACGT core derived motifs it had been shown that in particular group $\mathrm{S}$ bZIP proteins also recognize additional cis-elements, like the ACTCAT motif in the promoter of the prolin-dehydrogenase (ProDH) gene (Satoh et al., 2004; de Pater et al., 1994).

In Arabidopsis the group S AtbZIP-TFs represents the largest group of homologous proteins within the AtbZIP TF family (Jakoby et al., 2002). It consists of 17 small (S) TF proteins, from which each is encoded by an intronless gene. In general, group $\mathrm{S}$ bZIPs exhibit a molecular weight of 15 to $20 \mathrm{kDA}$ and are composed of a short $\mathrm{N}$ terminus, a central basic domain, an unusual long leucine zipper dimerisation domain of eight to nine heptad repeats and a short C-terminal extension (Jakoby et al., 2002). 
Members of this group can be, based on sequence homology of their DNA-binding domain and additional conserved motifs, further classified into 3 subgroups, denoted S1 to S3 (Jakoby et al., 2002). The subgroup S1 consists of five AtbZIP-TFs, namely AtbZIP1, 2, 11, 44 and 53. Besides their high protein homology, they are characterised by an exceptionally long $\underline{\mathbf{5}}$ untranslated region ( $5^{\prime}$ UTR) which harbours an upstream open reading frame (uORF) (Rook et al., 1998). This uORF was found to mediate a post-transcriptional repression mechanism operating in all S1 homologous bZIP proteins (reviewed in Smeekens et al., 2010). As this regulatory system has been demonstrated to repress translation of the main bZIP ORF in a sucrose concentration dependent manner, by a yet uncharacterised ribosome stalling step, it was designated as “sucrose induced repression of translation" (SIRT) mechanism (Rook et al., 1998; Wiese et al., 2004; Weltmeier et al., 2009).

In several plant species, homologs of group S AtbZIPs have been found to specifically and preferentially form heterodimers with group C bZIP-TFs, which suggests that these two groups are functionally interlinked (Alonso et al., 2009, Weltmeier et al., 2006; Strathmann et al., 2001; Pysh et al., 1993; Rugner et al., 2001). The group C of Arabidopsis bZIP-TFs is composed of four related proteins, namely AtbZIP9, 10, 25 and 63, which similarly to group S AtbZIPs, possess an extended leucine zipper dimerisation domain of eight heptad repeats. In contrast to group $\mathrm{S}$, group $\mathrm{C}$ AtbZIPs exhibit a roughly twice as high molecular weight of about 30 to $40 \mathrm{kDA}$ (Jakoby et al., 2002) and harbour multiple putative phosphorylation sites in their N-terminal region. On genomic level it is apparent, that all group C AtbZIPs display a conserved intronexon structure (Heinekamp et al., 2002).

Recent findings indicated that specific AtbZIP-TFs of both groups constitute a corporately acting C/S1 TF network which is involved in translating the prevailing energy status of the plant into adaptive metabolic reprogramming (reviewed in Smeekens et al., 2010). In this respect it has been demonstrated that specific group C and S1 AtbZIPs are crucial regulators of the co-ordinated adaptation of the amino acid and carbohydrate metabolism under energy deprived conditions (Dietrich et al., 2011; Hanson et al., 2008; Weltmeier et al., 2006; Smeekens, 2010). Complementary, results obtained from transient protoplast transfection assays revealed that the expression promoting activity of specific group C and S1 AtbZIPs on the promoter of the 
asparagine synthase 1 (ASN1) gene, which is a key player in adaptive amino acid metabolism during energy starvation, could be substantially enhanced by co-expression of the Arabidopsis SnRK1 kinases, AtKIN10 or AtKIN11 (Baena-Gonzalez et al., 2007). These kinases, which are activated upon nutrient starvation, are known to be central integrators of stress and energy signalling and have been implicated in extensive, adaptive reprogramming of gene transcription and plant growth control (Smeekens et al., 2010; Thelander et al., 2004; Radchuk et al., 2006; Baena-Gonzalez et al., 2007, 2008). Moreover it has been presented that constitutive expression of the group S1 AtbZIP-TFs, in particular AtbZIP2, 11, 44 and 53 results, depending on the bZIP expressed, in a moderate to strong impairment of plant growth, indicating that these bZIPs might have a growth regulatory function. As the endogenous expression of these AtbZIPs is in addition closely intertwined with the plants energy status via the sucrose concentration dependent SIRT mechanism, it has been postulated that specific group S1 AtbZIPs might adapt plant growth to fluctuating energy conditions (Rahmani et al., 2009; Weltmeier et al., 2009).

As many growth-related responses could be ascribed to the plant hormone auxin and tobacco homologs of group C and group S1 AtbZIP-TF, have been implicated in altering auxin mediated responses (Heinekamp et al., 2004) it is of great interest to define if and how these energy-stress related AtbZIP-TFs might constitute a regulatory framework to intervene in auxin- and energy-signalling. This would shed light on the issue how plants are able to adapt their growth and primary metabolism to the prevailing energy status of the plant.

\subsection{Outline and objectives of the thesis}

In order to enable optimal plant development under continuously changing environmental conditions, the plant has to adjust its metabolism and growth to the prevailing energy status. As a wide range of growth and developmental processes, but also responses to environmental cues are controlled by the plant hormone auxin, it has been postulated that internal and external stimuli need to be integrated into auxin mediated processes. Auxin predominantly exerts its function through auxin mediated gene expression, which is suggested to be orchestrated by a combinatorial 
transcriptional control by ARF and AUX/IAA proteins. Recent findings indicate that besides these two established classes of auxin related TFs, also members of other TF families, such as specific MYB-TFs might be capable to considerably modulate auxin responsive gene expression and their related responses. Previous work from our group has indicated that two tobacco bZIP-TFs are also involved in altering auxin mediated expression and plant growth. However a detailed functional and mechanistical characterisation of bZIP-TF action on auxin mediated transcription hasn`t been addressed, yet. In the well-established dicot model plant Arabidopsis thaliana the closest homologs of these tobacco bZIPs belong to the C/S1 network of AtbZIP-TFs. Making use of the molecular tools available for this model plant various approaches have been applied to further characterise the relevance of bZIP transcription factors on auxin mediated plant responses.

Chapter 1: Comprehensive bioinformatic promoter cis-element analyses were applied to determine the distribution and abundance of specific AtbZIP- and MYB-TF related binding sites in auxin responsive promoters from the dicot and monocot model plants Arabidopsis and rice, respectively. Most noticeable, both evolutionary long separated species exhibited a conserved enrichment of single and composite modules of bZIP- and ARF-TF related binding sites in especially auxin inducible promoters.

Chapter 2: The functional relevance of the most enriched bZIP-TF cis-element in auxin responsive promoters was defined by mutational promoter analyses in transient protoplast transfection assays, by utilising the early auxin responsive AtGH3.3 promoter as a model system. By this means it could be presented that the bZIP-TF associated Gbox related cis-element (GRE) constitutes a quantitative coupling motif of the auxin responsive element (AuxRE). In transient trans-activation screening approaches, specific group S1 AtbZIP-TFs were identified, which were capable to strongly induce various auxin responsive promoters. Complementary gain- and loss-of-function approaches in transgenic plants demonstrated that, in particular AtbZIP11-related TFs are able to modulate both, auxin responsive gene expression and auxin related growth responses. 
Chapter 3: In order to define the underlying mechanism of the expression promoting property of group S1 AtbZIP-TFs on auxin responsive genes, the ability and relevance of AtbZIP co-factor recruitment was analysed. In fact, it could be demonstrated that AtbZIP11-related TFs are capable to bind the adapter components of an Arabidopsis SAGA-like HAT complex. Pharmacological, reverse genetic and immunological approaches were applied to demonstrate that the activation potential of AtbZIP-TFs on auxin mediated transcription can be ascribed to the recruitment of HAT complexes to the auxin responsive bZIP target promoters. Accordingly it can be postulated that these AtbZIPs establish a counteracting system to the AUX/IAA repressor proteins, by HAT mediated de-condensation of the chromatin, which facilitates RNA Polymerase II binding and hence transcription.

Chapter 4: The expression of all group S1 AtbZIP-TFs is transcriptionally and posttranscriptionally controlled by intracellular sucrose levels and is thus directly intertwinded to the prevailing energy status of the plant. It has been demonstrated that specific group S1 AtbZIPs are, besides their role in modulating auxin mediated gene expression and auxin-related growth responses, also involved in adaptive reprogramming of the amino acid metabolism under energy deprived conditions. This suggests that group S1 AtbZIPs might constitute a regulatory system to adjust plant metabolism and growth according to the energy supplies of the plant. 


\section{$3 \quad$ Results}

3.1 Chapter 1: Bioinformatic cis-element analysis performed in Arabidopsis and rice reveals bZIP- and MYB-related binding sites as coupling elements in AuxRE mediated auxin responsive transcription*

* Parts of this manuscript are prepared for publication

Kenneth W. Berendzen*a ${ }^{\mathrm{a}}$, Christoph Weiste ${ }^{\mathrm{b}^{\mathrm{c}} \mathrm{c}}$, Dierk Wanke ${ }^{\mathrm{a}}$, Joachim Kilian ${ }^{\mathrm{a}}$, Klaus Harter ${ }^{\dagger a}$ and Wolfgang Dröge-Laser ${ }^{\dagger b, c}$

${ }^{\mathrm{a}}$ Zentrum für Molekularbiologie der Pflanzen, Pflanzenphysiologie, Universität Tübigen, Auf der Morgenstelle 1, 72076 Tübingen, Germany

b Albrecht-von-Haller Institut, Universität Göttingen, Untere Karspüle 2, 37073 Göttingen, Germany

c Julius-von-Sachs-Institut, Pharmazeutische Biologie, Universität Würzburg, Julius-von-Sachs-Platz 2, 97082 Würzburg, Germany

* joint first-authors

$\dagger$ joint corresponding authors, correspondence should be addressed to:

Wolfgang Dröge-Laser, e-mail: wolfgang.droege-laser@uni-wuerzburg.de Klaus Harter, e-mail: klaus.harter@zmbp.uni-tuebingen.de 


\section{Abstract}

\section{Background:}

In higher plants, a diverse array of developmental and growth-related processes is regulated by the plant hormone auxin. Recent publications revealed that besides the well-characterized $\underline{\text { Auxin }}$ Response Factors (ARFs) which bind auxin response elements (AuxREs), also members of the bZIP- and MYB-transcription factor (TF) families are able to modulate the expression of auxin-regulated genes via bZIP related elements (BREs) or Myb related elements (MREs), respectively. To examine whether a combinatorial regulation of auxin mediated transcription by these TF-classes is of general importance, genome wide cis-element analyses have been performed to determine the frequency and distribution of ARF-, bZIP- and MYB-binding sites and composite modules of these motifs in monocot (Oryza sativa) and dicot (Arabidopsis thaliana) model plants.

\section{Results:}

Applying a novel bioinformatic algorithm, we could demonstrate that singular and composite modules of AuxREs, BREs and MREs are significantly enriched in promoters of auxin-inducible genes, suggesting that these motifs act in a co-operative manner. Furthermore, an enrichment of defined composite modules has been observed in selected auxin-related gene families. Although their species specific genome structure, in terms of the GC content is considerably different, this enrichment is generally conserved in both angiosperm plant species tested. Consistently, a bipartite GRE-AuxRE module, which encompasses the bZIP-TF associated $\underline{\text { G-box }}$ related element (GRE) and an AuxRE-motif, has been found to be highly enriched. Making use of transient reporter studies in protoplast, this module was confirmed to mediate auxininduced transcription, whereas the quantitative level of induction was strongly diminished in reporter constructs lacking the functional GRE-motifs.

\section{Conclusions:}

These observations strongly suggest that auxin-induced, AuxRE-mediated transcription is further fine-tuned by cooperation with specific bZIP- and MYB-binding sites. Furthermore, an evolutionary conserved regulatory mechanism is proposed using ciselement modules to establish specific auxin- induced expression patterns. 


\section{Background:}

Auxin is a major plant hormone that regulates controlled plant growth as it is involved in diverse plant developmental processes (Moller and Weijers, 2009), such as apical dominance (Leyser, 2005), root formation (Bennett and Scheres, 2010) and growthrelated tropisms, like phototropism and gravitropism (Muday, 2001). In general, the manifestation of auxin mediated responses is ascribed to the encoded activity of auxin responsive genes (Vanneste and Friml, 2009). Although comprehensive microarray analyses have enabled the identification and classification of auxin responsive genes according to their individual auxin mediated induction kinetics (Goda et al., 2004, Jain and Khurana, 2009) it is still largely unknown which regulatory elements could provide means to modulate their individual expression patterns in particular to internal and external stimuli. The transcriptional response to auxin is primarily mediated through cis-regulatory Auxin Response Elements (AuxREs) (Ulmasov et al., 1999c). These elements are bound by $\underline{\text { Auxin }}$ Response Factors (ARF) (Ulmasov et al., 1997a) that act with Aux/IAA proteins to regulate auxin dependent gene transcription, whereby Aux/IAA proteins repress ARF activity at low cellular auxin concentrations (Guilfoyle et al., 1998a, Guilfoyle et al., 1998b, Gray et al., 2001). As auxin mediates the interaction of AUX/IAA repressor proteins with the $\mathrm{SCF}^{\mathrm{TIR} 1}$ auxin receptor complex, that marks the repressor proteins for degradation by the $26 \mathrm{~S}$ proteasome, increasing auxin concentrations lead to a de-repression of ARF-TF target genes (Dharmasiri et al., 2005b, Kepinski and Leyser, 2005b).

AuxREs were first discovered and characterized through foot-printing analysis within the PSIAA4/5 promoter of Pisum sativum (Ballas et al., 1993). Later, they were shown to function in a soybean GH3 promoter (Liu et al., 1994, Ulmasov et al., 1995). Although it could be demonstrated that AuxREs are sufficient to provide auxinresponsiveness (Ulmasov et al., 1995, Ulmasov et al., 1997a, Ulmasov et al., 1997b), they frequently occur and function as composite elements in a genuine promoter context (Guilfoyle et al., 1998a, Hagen and Guilfoyle, 2002). Therefore it has been postulated that AuxRE coupling elements might have a modulating effect in the transcriptional response to auxin (Ulmasov et al., 1995).

Indeed, quite recently it was pointed out, that a member of the Arabidopsis R2R3 MYB transcription factor (TF) family, namely AtMYB77, interacts with AtARF7 and 
synergistically promotes the expression of an auxin-responsive reporter construct (Shin et al., 2007). In line with these findings Arabidopsis myb77 plants exhibit a downregulation of several auxin-responsive genes (IAA1, IAA19, PIN1, GH3.2, GH3.3,

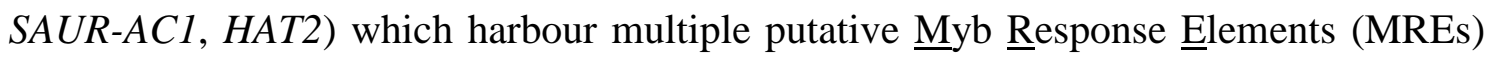
in their promoters. In comparison to wild-type (wt) plants, myb77 and the auxin receptor mutant tirl-1 exhibit a similar decrease in auxin controlled lateral root density under potassium-deprived conditions. Hence, the authors concluded that AtMYB77 plays a role in altering auxin responses during transition from nutrient-sufficient to -deficient conditions by presumably modulating the plant's sensitivity to auxin (Shin et al., 2007).

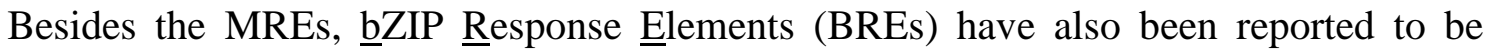
potential quantitative elements in auxin mediated transcription. The well-characterised auxin-responsive soybean $G m G H 3$ promoter for instance, contains three composite units, encompassing AuxREs and adjacent or partially overlapping G-box Related Elements (GRE). EMSA studies confirmed that a recombinant G-box specific basic leucine zipper (bZIP) TF can bind these GREs (Ulmasov et al., 1995, Liu et al., 1997a). A similar promoter organisation can also be found for the auxin-responsive GmAux28 gene in which the GREs are bound by two soybean G-box binding factors, SGBF-1 and SGBF-2 (Nagao et al., 1993, Hong et al., 1995), which however are not functionally characterized, yet. In tobacco, AuxRE and GRE composed modules were located in the promoter of the $N t G H 3$ gene and at least two GREs were identified that were recognised by the NtBZI-1 TF which promotes NtGH3 transcription in an auxin dependent manner (Heinekamp et al., 2004).

Despite these observations it is yet unknown, whether modules of AuxRE, BRE and MRE cis-elements frequently occur in auxin-responsive promoters and thus participate in a common regulatory mechanism in auxin mediated transcription. To address this question, we conducted a genome-wide bioinformatic analysis of auxin responsive promoters in a dicot (Arabidopsis thaliana) and monocot (Oryza sativa) model plant. These analyses confirmed that specific singular and composed modules, consisting of AuxREs, BREs and MREs are significantly enriched in the promoters of auxin inducible genes and specifically in some auxin-regulated gene families. In particular, enrichment of a GRE-AuxRE module was found. Using protoplast transactivation 
assays, we experimentally demonstrated the relevance of GREs as quantitative modulators of auxin-induced, AuxRE-mediated transcription.

\section{Results}

\section{Promoters of $\mathrm{GmGH3}$ homologs from diverse monocot and dicot plant species exhibit several BRE and MRE motifs in the proximity of AuxREs}

Previous studies have suggested that BREs and MREs might play a considerable role in the regulation of some auxin inducible GH3 promoters (Hagen et al., 1991, Liu et al., 1994, Heinekamp et al., 2004, Shin et al., 2007). Especially GRE and TGA motifs (see Table 1), which are bound by G-box binding factors (Schindler et al., 1992, Menkens and Cashmore, 1994), were frequently found to reside near AuxREs. Similar observations were made for MREs with respect to the Arabidopsis AtGH3.2 and AtGH3.3 genes. The promoters of these genes contain several MRE motifs and their expression is significantly decreased in myb77 plants (Shin et al., 2007).

In order to elucidate whether this phenomenon is specific for these genes or might be a general feature of early auxin-responsive $G H 3$ promoters we identified homologs of the soybean $G m G H 3$ in several monocot and dicot plant species and analysed the distribution of AuxREs, BREs and MREs in their corresponding promoters. Except of soybean, homologs were found in seven other angiosperm plant species: the monocots (Oryza sativa, Sorghum bicolour, Zea mays) and the dicots (Arabidopsis thaliana, Glycine max, Lotus japonicus, Medicago truncatula, Vitis vinifera). For the homology search, considerably low BLAST scores $\left(\mathrm{p} \leq 1 \times 10^{-5}\right)$ were chosen to keep the number of putative homologs as limited as possible and to restrict the dataset to likely ortholog and paralog candidates. On the basis of the GH3 protein sequences, a neighbour-joining phylogram was created using GmGH3 as outgroup. Their corresponding promoters (1000 to $-1 \mathrm{bp}$ ) were then scanned on both strands for the consensus AuxRE motif (TGTCTC core sequence) (Ulmasov et al., 1999b) which we call AUX1 and its less stringent variant AUX2 (TGWCTS core sequence) (Ballas et al., 1993, Abel et al., 1996), three different BREs: GRE-, TGA- and the AC-motif (ACTCAT core sequence) which was found to be bound by AtbZIP-TFs (Schindler et al., 1992, Menkens and Cashmore, 1994, Satoh et al., 2004), and two MREs: MRE1 (AMCWAMC core 
sequence) and MRE2 (GGWTW core sequence) (Abe et al., 1997, Martin and Paz-Ares, 1997, Romero et al., 1998, Chen et al., 2002) (see Table 1).

Table 1: Overview of TF-binding sites used in the promoter cis-element analyses. Cis-elements with envisaged role in auxin mediated transcription were complied and organized into three classes: bZIP-TF related elements (BREs), B3-type TF related elements (AUXs/RY) and MYB/MYC-related elements (MREs/MYC2).

\begin{tabular}{|l|c|c|l|}
\hline & Abbr. & Sequence & Putative trans-acting factors \\
\hline bZIP related binding sites & & & \\
\hline G-box related & GRE & BACGTV & bZIPs \\
\hline TGA element & TGA & TGACG & bZIPs (group D) \\
\hline ACTCAT element & AC & ACTCAT & bZIPs (some group S) \\
\hline & & & \\
\hline B3-type related binding sites & & & \\
\hline AuxRE & AUX1 & TGTCTC & B3-type (ARFs) \\
\hline AuxRE-related & AUX2 & TGWCTS & B3-type (ARFs) \\
\hline Sph/RY & RY & CATGCATG & B3-type (e. g. ABI3) \\
\hline & & & \\
\hline Myb/Myc binding sites & & & \\
\hline Myb-related element 1 & MRE1 & GGWTW & MYBs \\
\hline Myb-related element 2 & MRE1 & AMCWAMC & MYBs \\
\hline Myc-related element & MYC2 & CACATG & MYCs \\
\hline
\end{tabular}

The resulting phylogram revealed that the analysed MRE motifs were frequently distributed throughout the $G H 3$ promoters tested. Especially, the MRE2 motif occured at a very high frequency and at least once in every promoter, whereas the MRE1, which was still present in a relevant proportion of them $(\sim 75 \%)$, showed a much lower abundance (Figure 1). Concerning the distribution of the BREs it could be observed that the majority of the promoters of the GmGH3 homologs contained one or more BRE and only a small number $(\sim 13 \%)$ did not contain any of the BREs tested. Furthermore, it was quite remarkable that a correlation between protein homology and promoter organisation with respect to BRE and AuxRE motif abundance could be observed. More precisely, closely related $G H 3$ proteins could be separated in two more or less distinct clades according to the occurrence of BREs in their corresponding promoter sequences. The only exception to this were promoters from two GH3 homologs from Lotus japonicus (LjCM0124) and Vitis vinifera (Vv0006220001) that were found from protein 
homology within the envisaged BRE rich clade (clade II) but should be located in the BRE poor clade (clade I) as their corresponding promoters did not contain any BRE (Figure 1). Moreover, it is mentionable that GH3 homologs from each analysed plant species had representatives within both clades, demonstrating that the corresponding genomes encode for $\mathrm{GH} 3 \mathrm{~s}$ which associated promoters are either rich or poor of BRE motifs. A closer inspection of BRE abundance showed that GRE- and TGA-motifs are the most prominent BREs, whereas the AC-motif occured relatively rare in the promoters but also with a higher frequency in the BRE rich clade. Finally it should be considered that all BREs were often found near AuxRE motifs (Figure 1).

In summary the results from this analysis demonstrate that within conserved GH3 protein subclasses, a conservation of distribution and abundance of specifc AuxRE, BRE and MRE cis-elements can be observed. This indicates that in particular, GRE, TGA and MRE1/2 motifs might co-operate with AuxREs in the regulation of auxinrelated group II GH3 genes. In order to examine if this clustering of AuxRE, BRE and MRE motifs is in general evolutionary conserved in auxin-responsive promoters we analysed their abundance and distribution on a genome-wide basis. As a prerequisite, a real randomization algorithm for the Motif Mapper software was designed to create randomized control promoter datasets, for comparison and subsequent statistical analysis, to identify truly enriched motifs and motif combinations in large promoter datasets. 


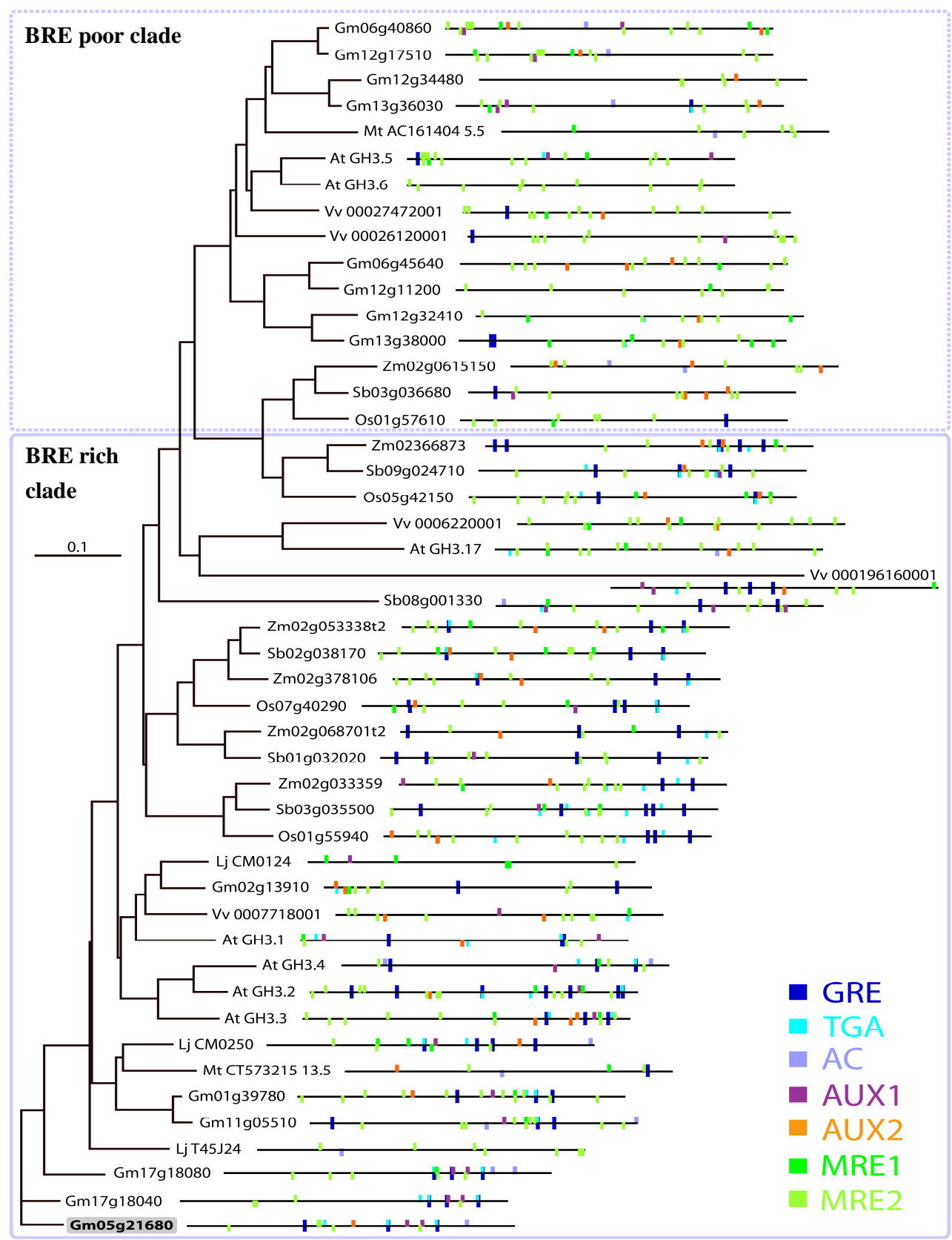

Figure 1: Phylogram of group II GH3 homologs to the soybean GH3 Gm05g21680. The closest homologs to the well-characterized soybean GH3 (Gm05g21680) (Staswick et al., 2005) from several plant species were compiled and the corresponding predicted protein sequences were rooted to Gm05g21680 (at the bottom; grey highlighted) to create a neighbour-joining phylogram. The $1000 \mathrm{bp}$ promoter sequences of the corresponding $G H 3$ genes were plotted, in 5' to 3' orientation, onto the phyolgram presenting specific BRE, MRE and AuxRE cis-elements (see Table 1) detected in their promoters. The bordering boxes divide the phylogram into the BRE-rich (lower section; continous line) and BRE-poor (upper section; dashed line) clades. The scale reflects the number of amino acid substitutions per site. 
A real randomization algorithm was designed to detect significantly enriched and depleted single and combined cis-element modules in specific promoter datasets

The determination of significance for an enrichment or depletion of certain cis-elements or cis-element module combinations in a given dataset is quite challenging. The difficulty arises in how to define the number of cis-elements and modules as accurate as possible. Many algorithms are available, capable of searching de novo or using user defined modules (Van Loo and Marynen, 2009). Some function reliably with metazoans as algorithm, e.g. TOUCAN2 (Aerts et al., 2005) while others require clusters of coexpressed genes. However, we headed for a simple, but effective method for testing the significant occurrence of various motifs and modules at the same time in unprocessed lists of genes without prior clustering. Therefore, we decided to test for motif and module enrichment in comparison to a real randomized, genomic promoter dataset for each individual species without superimposed modelling. The features of this algorithm are presented below and are integrated into the graphical interface version of Motif Mapper (see methods).

Module description - We used word matching while allowing alternative bases to be represented by International Union of Pure and Applied Chemistry (IUPAC) letters (Cornish-Bowden, 1985). Modules can be of any number of motifs, with any defined or flexible spacing between them. Previous work has suggested that some cis-motifs have a 5' to 3' bias with respect to the transcriptional start site of a gene (Berendzen et al., 2006, Brown et al., 2007) but the full significance for TF recognition is still unknown. In order to explore if composite modules could also have a 5' to 3 ' bias, cis-element modules were analysed in both orientations, while allowing the single embedded cismotifs to be identified on both strands as Watson or Crick words. In contrast to other bioinformatic approaches, we do not have to extrapolate the relationships between multiple motifs a priori. For any set of genes of size $n$, the algorithm extracts a random cluster of size $\mathrm{n}$ for any number of repetitions. We found that 1000 random extractions yielded reliable results in a reasonable amount of time. Using this approach it is possible to calculate the significance of four parameters simultaneously: (I) "the number of promoters with a motif" (Figure 2A); (II) "number of motifs per promoter" (Figure 2B); (III) "the total number of motifs" and (IV) "the motif variance of promoters with a 
motif", which indicates whether or not a specific motif is equally abundant in the promoter dataset. The algorithm output includes three dataset values: the actual input dataset, the average of the random datasets, and the p-values for the four parameters mentioned above. The random sampling is sufficient to yield Gaussian background distributions from which p-values can be effectively calculated using a Z-score. Since a complete genomic population distribution is present, this method can be used to calculate for both enrichment and depletion. For illustration, some dataset parameters for the GRE motif are shown in Figure 2 A, $\mathbf{B}$ with respect to its random background distribution.

False-positive error rate - To evaluate the quality of the randomization process, the algorithm enables the user to return both, the randomization indices (Figure 2A, B, C) and the parameters (random background distribution values, z-comparison values) used for calculating the p-values. The randomization algorithm was able to deliver Gaussian distributions for the analysed motifs from the background (Figure 2A, B) and overall, most individual promoters were called only 1 to 3 times (Figure 2C), indicating that the sampling of the genomic dataset was sufficient. The false-positive error rate was calculated by screening 1000 random times, randomly composed datasets of various sample sizes (50, 200 and 1000 promoters) for various motifs and calculating the probability that a given motif is termed enriched by mistake. Figure 2D shows the false-positive error rate for two p-value cut-offs (0.1 and 0.01) for the GRE, AuxRE and two MRE motifs in the Arabidopsis genomic promoter dataset. Using this method, a pvalue cut-off of 0.1 is too generous as it has a false-positive error rate around 0.3. Reducing the p-value call for significance to 0.01 however, reduced the false-positive error rate to 0.05 or below for most motifs. The nature of the motif and the sample size can lead to larger false-positive error rates. For example, the MRE2 motif exhibits a higher false-positive error rate for larger dataset sizes when scoring the parameter "promoters with a motif", as it is a very common cis-element that occurs in nearly every promoter in Arabidopsis. 
A

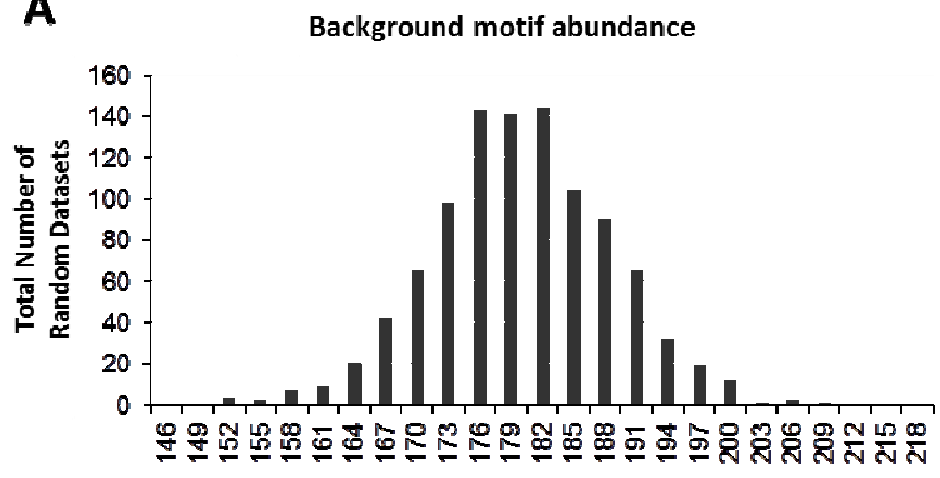

Number of Promoters with Motif

\section{C}

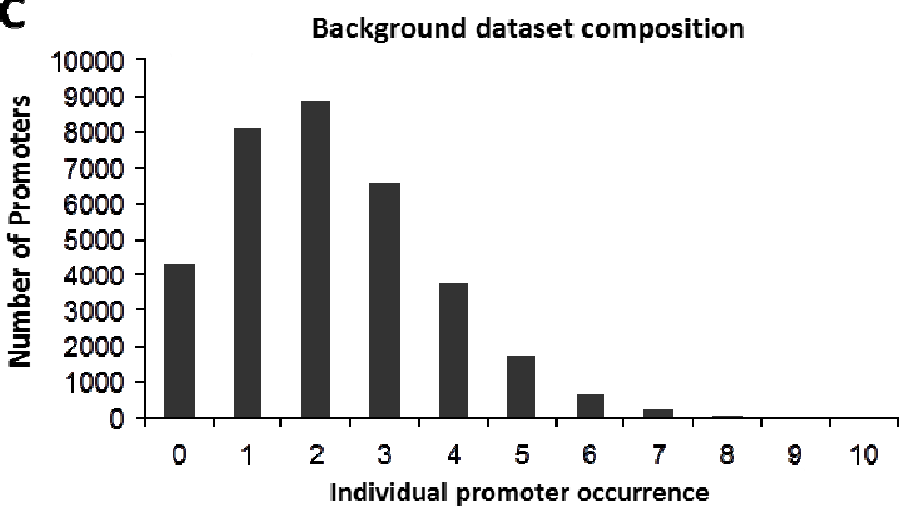

B

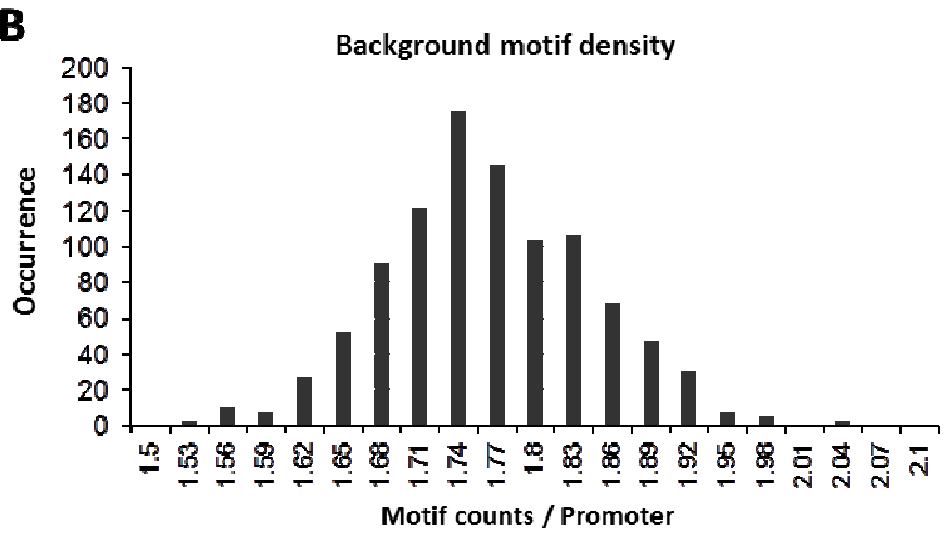

D

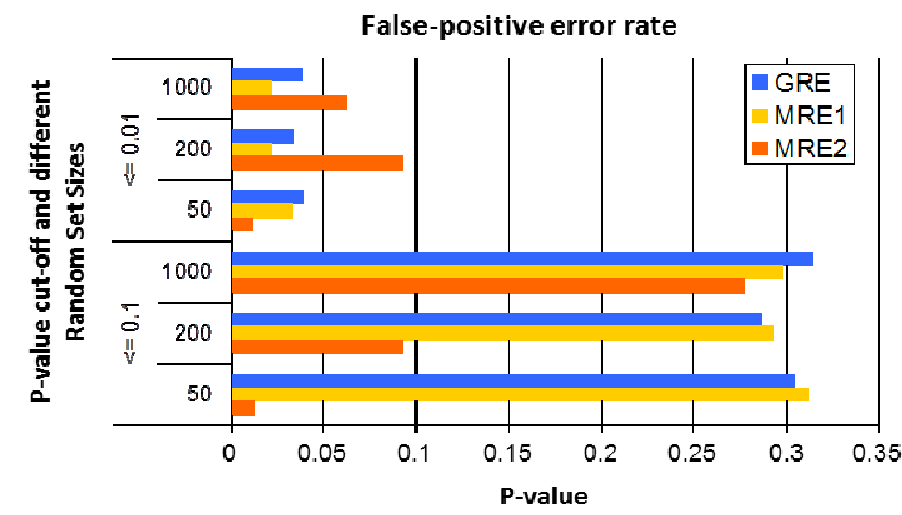

Figure 2: Typical output of the applied randomization algorithm. A) The parameter "number of promoters with a motif" was exemplarily determined for the GRE motif in several randomized promoter datasets (1000 random sets of 304 genes) and its distribution is visualised in the given histogram. Experimental datasets which exhibit a significant enrichment or depletion for e.g. the GRE motif should display a respective substantially shifted distribution pattern to that of the presented background distribution. B) The parameter "motif counts per promoter" was exemplarily determined for the GRE motif in several randomized promoter datasets (1000 random sets of 304 genes) and the average number of motif counts per promoter is visualised in the given histogram. Experimental datasets which show an enriched or depleted motif density in the promoters should display a respective shifted motif density pattern to that of the presented background density. C) Illustration of the number of times a promoter was randomly pulled to participate in a random dataset. The algorithm pulls individual promoters from the genomic dataset only once or twice, indicating that only very limited redundancy is present in the background dataset modelling. D) Excerpt of the false-positive error rate calculations for the parameter "number of promoters with a motif". Exemplarily the error-rates for genomic frequent and infrequent motifs used in this study are given. As the false positive error-rate is dependent on the dataset size, it was calculated for each motif in differently sized random datasets (50, 200, 1000). The given false-positive error rate is an average value from 1000 calculation repetitions. Reducing the p-value call for significance to 0.01 , reduced the false-positive error rate for most analysed motifs to 0.05 or below. 
The number of promoters with a motif is in general the most reliable significance parameter - It is in principle assumed that a set of genes which are corporately regulated by the same type of TF, requires that their promoters posses the corresponding TF-binding sites. Furthermore we assume that those cis-elements should be in either all or a significant proportion of the TF regulated promoters. In accordance with this, we observed that the parameter "number of promoters with a motif" carries the most significant relation for cis-element enrichment or depletion in a specific dataset. In the exceptional case that a motif is highly abundant in the analysed genomic promoter dataset (e. g. MRE2 as described in the previous section) the parameter "number of motifs per promoter" could be used additionally for a particular dataset as it can be more meaningful regarding an enrichment or depletion of such a cis-element. For that reason we drew our attention in the majority of cis-element distribution analyses to the parameter "number of promoters with a motif" as the most relevant observation parameter with a significance call of $\leq 0.02$ and a false-positive error rate of $\leq 0.06$, but if necessary also presented the parameter "number of motifs per promoter" as positive or negative motif density for highly abundant motifs.

\section{Bioinformatic cis-element analysis of auxin-responsive promoters from Arabidopsis reveals that auxin inducible, but not repressible promoters are enriched for specific composite cis-element modules}

To analyse the distribution of AuxRE, BRE and MRE cis-elements in auxin-responsive promoters on a genome-wide scale we made use of the publicly available AtGenExpress Arabidopsis microarray data (see methods), to initially identify auxin-regulated genes. The microarray data applied, was part of an auxin time course experiment with 7-daysold Arabidopsis Col-0 wt seedlings. Samples from mock and $1 \mu \mathrm{M}$ IAA treated plants were taken at $0.5,1$ and $3 \mathrm{~h}$ after treatment onset. After normalization, genes 2-fold induced or repressed compared to the controls were identified for each time point and for nearly every regulated gene call the corresponding promoter sequences were assignable, giving the obtained promoter dataset good coverage. Subsequently we grouped the promoter sequences into 6 classes, taking into account if their corresponding genes were up- or down-regulated after the different induction timepoints. The resulting groups were then scanned for the presence of specific AuxREs, 
BREs and MREs (Table 1) and their significant enrichment or depletion was determined using the previously described real randomization algorithm. The list of ciselements which was already used in the promoter cis-element analysis of the GH3 promoters was expanded for the genome-wide analysis by the RY motif (CATGCATG sequence) which is bound by ARF-like B3-type TFs (Dickinson et al., 1988) and a MYC2 TF binding site (CACA-TG) which strongly resembles a consensus G-box (CACGTG) which is bound by bZIP-TFs (Menkens and Cashmore, 1994, Abe et al., 1997). Concerning module compositions, all possible combinations between members of the AuxRE-, BRE- and MRE/MYC-motif classes were tested and the distance between each individual cis-motif was restricted to a maximum of $100 \mathrm{bps}$, since the majority of composite elements that have been described so far were either overlapping or very close to each other (Ulmasov et al., 1995, Hagen and Guilfoyle, 2002).

Figure 3A - C illustrates the results from the cis-element analysis. Most noticeable was that the promoters from the auxin up-regulated genes were disproportionably more enriched for single motifs and bipartite modules of AuxRE-, GRE- and MYC2-motifs compared to the promoters of the down-regulated genes (Figure 3A, B). Particularly, the GRE-motif was found to be significantly enriched in the promoters of the auxin upregulated genes, 1 and $3 \mathrm{~h}$ post-treatment and moreover occured to a significant higher degree in association with the AUX1 and the less stringent AUX2 AuxRE motif in the early and late auxin inducible promoters (Figure 3A, B). Although the bipartite motif modules showed in general no orientation specificity to 5' or 3' positioning, the GREAuxRE module seemed to be preferential compared to the AuxRE-GRE module combination (Figure 3B). Considering that other BREs, like the TGA- and AC motif were per se and in combination with other motifs not enriched or even depleted at specific time-points (Figure 3A, B), it indicates that among all auxin up-regulated Arabidopsis promoters, the GRE motif seems to be the most preferred bZIP binding site.

Examining the distribution of the MREs, it became apparent that both single MRE ciselements; MRE1 and MRE2 were not significantly overrepresented in the auxinregulated promoters, whereas they occured with significant high frequency in combination with the GRE motif at 1 and $3 \mathrm{~h}$ post-treatment or all time points, respectively (Figure 3B). Moreover it was eye-catching that the MYC2 motif 
(CACATG) which is only present in $37 \%$ of all Arabidopsis promoters, was strongly enriched as single and as coupling element with both tested AuxRE motifs (Figure 3B).

As this site closely resembles a GRE, it remains to be disclosed which kind of TF binds in the context of auxin- induced transcription.

A Element

GRE
TGA
AC
AUX1
AUX2
RY
MRE1
MRE2
MYC2

B

Element
GRE-MRE1
GRE-MYC2
GRE-RY
GRE-MRE2
GRE-AUX1
GRE-AUX2
TGA-MRE1
TGA-MYC2
TGA-RY
TGA-MRE2
TGA-AUX1
TGA-AUX2
AC-MRE1
AC-MYC2
AC-RY
AC-MRE2
AC-AUX1
AC-AUX2
AUX1-AC
AUX1-MRE1
AUX1-GRE
AUX1-MYC2
AUX1-MRE2
AUX1-TGA
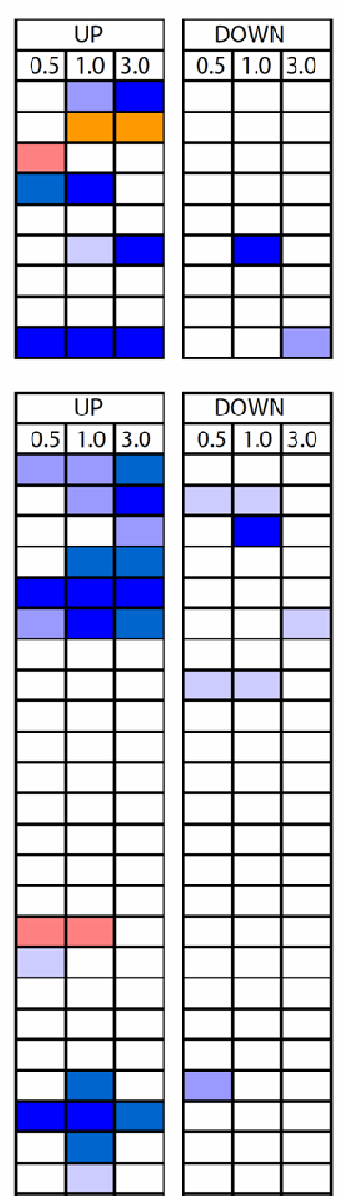
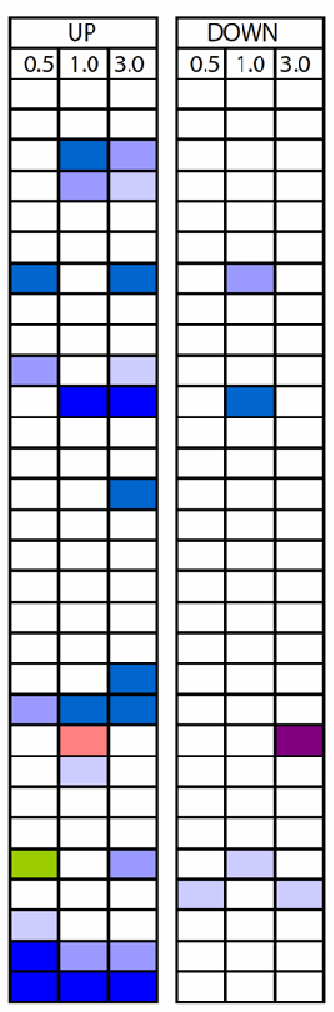

$B$

C

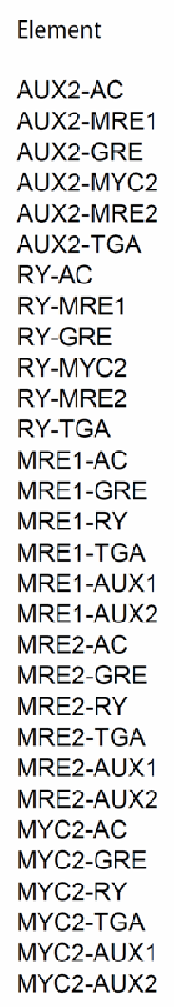
B Element
AUX2-MRE
AUX2-GRE
AUX2-MRE2
AUX2-TGA
RY-MYC2
RY-MRE2
RY-TGA
MRE1-RY
MRE2-AC
MRE2-GRE
MRE2-RY
MRE2-AUX2
MYC2-AC
MYC2-GRE
MYC2-TGA
MYC2-AUX2

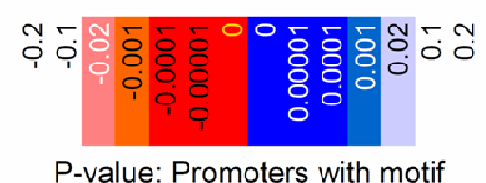


While Figure 3A, B illustrates which motif or module is enriched it does not determine which of the tested motifs is most abundant in the analysed dataset. Therefore, all significantly enriched motifs $(\mathrm{p} \leq 0.02$ ) were additionally ranked with respect to the quantity of promoters in which they occured. As MREs and GREs were both found to reside near AuxREs (Ulmasov et al., 1995, Shin et al., 2007) and might therefore synergistically contribute to auxin-mediated transcription we expanded the analysis and included triple motif modules of these three classes. The results from this evaluation are presented as a top 10 ranking list of single motifs or double and triple motif module combinations at each time point (Table 2) and are briefly summarized in the following remarks.

Table 2: Top 10 promoter-occurrence ranking list of the significantly enriched motifs and modules, within the early $(0.5-1 \mathrm{~h}$ post auxin treatment) and late ( $3 \mathrm{~h}$ post-treatment) auxin inducible promoters from Arabidopsis. Enrichment of cis-elements was determined by the parameter "number of promoters with a motif", whereas asterisks indicate an enriched with respect to "motif counts per promoter". Presented modules exhibit a variable but maximal spacing of 100 bps between each embedded motif.

\begin{tabular}{|c|c|c|c|c|c|c|}
\hline combination & RANK & $0.5 \mathrm{~h}$ UP & & $1 \mathrm{~h}$ UP & & 3 hours UP \\
\hline single & $\begin{array}{l}1 \\
2 \\
3 \\
4\end{array}$ & $\begin{array}{l}\text { AUX1 } \\
\text { MYC2 }\end{array}$ & $\begin{array}{l}1 \\
2 \\
3 \\
4\end{array}$ & $\begin{array}{l}\text { GRE } \\
\text { MYC2 } \\
\text { AUX1 } \\
\text { RY }\end{array}$ & $\begin{array}{l}1 \\
2 \\
3 \\
4\end{array}$ & $\begin{array}{l}\text { GRE } \\
\text { MYC2 } \\
\text { RY }\end{array}$ \\
\hline bipartite & $\begin{array}{c}1 \\
2 \\
3 \\
4 \\
5 \\
6 \\
7 \\
8 \\
9 \\
10 \\
\end{array}$ & $\begin{array}{l}\text { GRE-AUX2 } \\
\text { MYC2-AUX2 } \\
\text { GRE-MRE1 } \\
\text { GRE-AUX1 } \\
\text { AUX1-MYC2 } \\
\text { MYC2-GRE* } \\
\text { MYC2-AUX1 } \\
\text { MYC2-TGA } \\
\text { AC-AUX1 } \\
\text { MRE2-RY } \\
\end{array}$ & $\begin{array}{c}1 \\
2 \\
3 \\
4 \\
5 \\
6 \\
7 \\
8 \\
9 \\
10 \\
\end{array}$ & $\begin{array}{l}\text { GRE-MRE2 } \\
\text { AUX1-MRE2 } \\
\text { MRE2-AUX1 } \\
\text { GRE-AUX2 } \\
\text { AUX2-GRE } \\
\text { GRE-MRE1 } \\
\text { MYC2-AUX2 } \\
\text { GRE-AUX1 } \\
\text { AUX2-MYC2 } \\
\text { AUX1-GRE } \\
\end{array}$ & $\begin{array}{c}1 \\
2 \\
3 \\
4 \\
5 \\
6 \\
7 \\
8 \\
9 \\
10 \\
\end{array}$ & $\begin{array}{l}\text { MRE2-GRE } \\
\text { GRE-MRE2 } \\
\text { GRE-AUX2 } \\
\text { AUX2-GRE } \\
\text { MRE1-GRE } \\
\text { GRE-MRE1 } \\
\text { MYC2-AUX2 } \\
\text { GRE-MYC2 } \\
\text { AUX2-MYC2 } \\
\text { GRE-AUX1 } \\
\end{array}$ \\
\hline tripartite & $\begin{array}{c}1 \\
2 \\
3 \\
4 \\
5 \\
6 \\
7 \\
8 \\
9 \\
10 \\
\end{array}$ & $\begin{array}{l}\text { GRE-AUX2-MRE2 } \\
\text { MRE2-GRE-AUX2 } \\
\text { GRE-AUX2-MRE1 } \\
\text { AUX2-GRE-MRE1 } \\
\text { MYC2-AUX2-GRE } \\
\text { MYC2-TGA-AUX2 } \\
\text { GRE-MRE2-RY } \\
\text { RY-MRE2-GRE* } \\
\text { GRE-RY-MRE2* }^{*}\end{array}$ & $\begin{array}{c}1 \\
2 \\
3 \\
4 \\
5 \\
6 \\
7 \\
8 \\
9 \\
10 \\
\end{array}$ & $\begin{array}{l}\text { GRE-AUX2-MRE2 } \\
\text { GRE-MRE2-AUX2 } \\
\text { MRE2-AUX2-GRE } \\
\text { AUX2-GRE-MRE2 } \\
\text { AUX2-GRE-MRE1 } \\
\text { GRE-MYC2-AUX2 } \\
\text { MYC2-AUX2-GRE } \\
\text { MYC2-AUX2-TGA } \\
\text { AC-MYC2-AUX2 } \\
\text { TGA-MYC2-AUX2 }\end{array}$ & $\begin{array}{c}1 \\
2 \\
3 \\
4 \\
5 \\
6 \\
7 \\
8 \\
9 \\
10 \\
\end{array}$ & $\begin{array}{l}\text { GRE-AUX2-MRE2 } \\
\text { MRE2-AUX2-GRE } \\
\text { GRE-AUX2-MRE1 } \\
\text { GRE-MYC2-AUX2 } \\
\text { MYC2-AUX2-GRE } \\
\text { TGA-MYC2-AUX2 } \\
\text { GRE-MRE2-RY } \\
\text { RY-AC-MRE2 }\end{array}$ \\
\hline
\end{tabular}


Single motifs - Besides the well-described auxin response element (AUX1) and the relatively rare RY motif (only $3 \%$ in Arabidopsis promoters) that is bound by ARF like B3-type TFs, the GRE motif and its related MYC2 binding site are ranked within the top 4 most abundant cis-elements. The TGA motif which was shown to be frequently present in the promoters of the GmGH3 homologs was slightly depleted as a single motif within the set of all Arabidopsis auxin inducible promoters in this analysis. However it should be taken into account that this depletion was calculated on the basis of the parameter "number of promoters with a motif" compared to a randomized background dataset. Still a relevant number of promoters in the dataset of auxin inducible genes harboured this motif.

Bipartite motifs - Within the group of early auxin-responsive promoters $(0.5 \mathrm{~h}$ after IAA exposure) the GRE-AUX2 module was the best represented cis-regulatory unit as it ranked at position 1 and as GRE-AUX1 variant also on $4^{\text {th }}$ position. At later induction time points, it also ranked at high positions; $4^{\text {th }}$ and $8^{\text {th }}$ at $1 \mathrm{hr}$ and $4^{\text {th }}$ and $10^{\text {th }}$ at $3 \mathrm{hrs}$ post treatment but clearly behind the GRE-MRE2 module which was the most predominant module in the group of late induced promoters ( 1 and $3 \mathrm{~h}$ post-treatment). The GRE-MRE1 module should also be considered, as it remained among the top 10 list at all time-points $\left(3^{\text {rd }}\right.$ at $0.5 \mathrm{~h}$ and $6^{\text {th }}$ at 1 and $3 \mathrm{~h}$ post-treatment). The consensus AuxRE-motif AUX1 (TGTCTC) was the preferred binding site in the bipartite modules, within the promoters of the early auxin up-regulated genes $(0.5 \mathrm{~h}$ and $1 \mathrm{~h})$, although even more individual module combinations were identified using the relaxed AUX2motif. In agreement with this, recent publications suggest that the TGTCTC sequence is the preferred AuxRE even though variants are known (Hagen and Guilfoyle, 2002, Chapman and Estelle, 2009).

Tripartite motifs - Examining the distribution of modules of all three TF binding site classes, defined element combinations were identified that could be traced over the entire time series. The top ranking tripartite module was the GRE-AUX2-MRE2 at all time points. Thereafter, the GRE-AUX2-MRE1 as second best ranking module, followed by the GRE-AUX2-MYC2 combination. 
By summing up the results from this cis-element analysis of auxin-regulated promoters from Arabidopsis, it can be pointed out that single GRE motifs and their respective double and triple combinations with the analysed AuxRE and/or MRE motifs are significantly enriched within the group of the auxin-inducible promoters (Figure 3A, B;

Table 2). The observation that this enrichment is based on a frequent abundance of the GRE, GRE-AUX1/2, GRE-MRE1/2 as well as the GRE-AUX2-MRE1/2 containing modules in the analysed promoter sets, additionally affirms the relevance of these motifs in the regulation of auxin-inducible promoters.

\section{Cis-elements and modules of AuxREs, BREs and/or MREs are also enriched in auxin-inducible promoters from rice}

To assess whether the cis-element distribution, observed in the Arabidopsis auxin inducible promoters, is evolutionary conserved, we analysed a publicly available auxin induction microarray experiment from the monocot plant Oryza sativa. In the corresponding work (Jain and Khurana, 2009) transcript preparations from 7-days-old rice seedlings after an auxin treatment of 1 and $3 \mathrm{~h}$ were pooled and compared to the transcript sample of mock treated plants. Since the experiment thus contained no induction time series, only up- or down-regulated genes could be obtained. After normalization, these genes were classified with respect to a 2-fold expression difference due to the auxin treatment compared to the controls. Promoter sequences could be obtained for 223 up- and 110 down-regulated genes. The created promoter lists were then analyzed for the single, double and triple motif combinations as it had been done for the Arabidopsis promoters (Figure 4A - C; Table 3).

These analysis revealed that, although less of the analysed motifs and modules were found to be significantly enriched compared to Arabidopsis, the trend was preserved, that the promoters from the up-regulated genes harboured more enriched BRE-, MREor AuxRE-motifs and composite modules (22 in rice) compared to the down-regulated genes ( 3 in rice). Regarding the single motifs, the MRE2 and RY as well as GRE and TGA motifs were strongly enriched. For bipartite modules, the GRE-MRE2 or MRE1GRE as well as the AuxRE1/2-GRE were frequently abundant. These results are also reflected in the top ranking list in which the enriched motifs and modules were ranked 
according to the number of promoters they were present in (Table 3). In this list, the single MRE2-motif outranked all other motifs and was followed by the GRE- and TGAmotif on ranking position 2 and 3, respectively. Concerning the double motif modules, the GRE occured in 7 of the 22 possible bipartite modules and 5 of those were ranked in the top 10 list. Referring to this, the GRE-MRE2 and the TGA-MRE2 module on $1^{\text {st }}$ and $2^{\text {nd }}$ position together with the AuxRE-GRE module on $4^{\text {th }}$ position were the most prominent cis-elements. Within the group of the tripartite element modules the GREAUX2-MRE2 module was most preferred.

$\begin{array}{ll}\text { A } & \text { Element } \\ & \text { GRE } \\ \text { TGA } \\ \text { AC } \\ \text { AUX1 } \\ \text { AUX2 } \\ \text { RY } \\ \text { MRE1 } \\ \text { MRE2 } \\ \text { MYC2 } \\ \text { B Element } \\ \\ \text { GRE-AUX1 } \\ \text { GRE-AUX2 } \\ \text { GRE-RY } \\ \text { GRE-MRE1 } \\ \text { GRE-MRE2 } \\ \text { GRE-MYC2 } \\ \text { TGA-AUX1 } \\ \text { TGA-AUX2 } \\ \text { TGA-RY } \\ \text { TGA-MRE1 } \\ \text { TGA-MRE2 } \\ \text { TGA-MYC2 } \\ \text { AC-AUX1 } \\ \text { AC-AUX2 } \\ \text { AC-RY } \\ \text { AC-MRE1 } \\ \text { AC-MRE2 } \\ \text { AC-MYC2 } \\ \text { AUX1-MRE1 } \\ \text { AUX1-MRE2 } \\ \text { AUX1-MYC2 } \\ \text { AUX1-GRE } \\ \text { AUX1-TGA } \\ \text { AUX1-AC } \\ \text { AUX2-MRE1 } \\ \text { AUX2-MRE2 } \\ \end{array}$
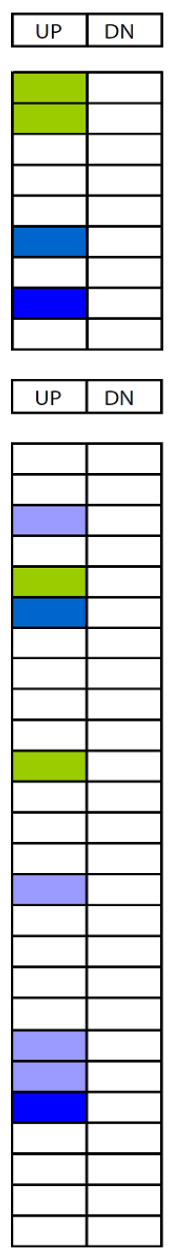

B

$$
\begin{aligned}
& \text { AUX2-MYC2 } \\
& \text { AUX2-GRE } \\
& \text { AUX2-TGA } \\
& \text { AUX2-AC } \\
& \text { RY-MRE1 } \\
& \text { RY-MRE2 } \\
& \text { RY-MYC2 } \\
& \text { RY-GRE } \\
& \text { RY-TGA } \\
& \text { RY-AC } \\
& \text { MRE1-AUX1 } \\
& \text { MRE1-AUX2 } \\
& \text { MRE1-RY } \\
& \text { MRE1-GRE } \\
& \text { MRE1-TGA } \\
& \text { MRE1-AC } \\
& \text { MRE2-AUX1 } \\
& \text { MRE2-AUX2 } \\
& \text { MRE2-RY } \\
& \text { MRE2-GRE } \\
& \text { MRE2-TGA } \\
& \text { MRE2-AC } \\
& \text { MYC2-AUX1 } \\
& \text { MYC2-AUX2 } \\
& \text { MYC2-RY } \\
& \text { MYC2-GRE } \\
& \text { MYC2-TGA } \\
& \text { MYC2-AC }
\end{aligned}
$$$$
\text { Element }
$$

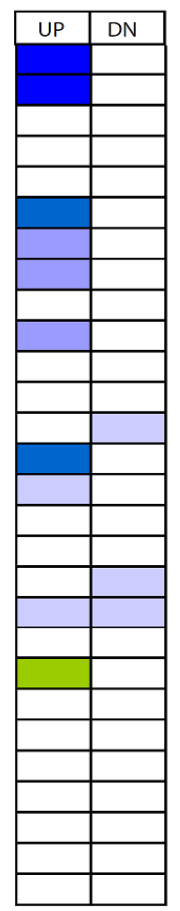

C

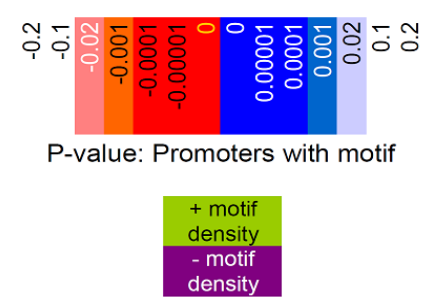

Figure 4: Statistical significance of motif enrichment or depletion for specific BRE, MRE and AuxRE related cis-elements in auxin responsive promoters from Oryza sativa. A) Motif enrichment or depletion for individual motifs in promoters of auxin-regulated genes. B) Significant enrichment or depletion of bipartite motif modules, of which embedded individual motifs have a variable, but maximal spacing of $100 \mathrm{bps}$, in promoters of auxin-regulated genes. C) The significance level scale for the parameters "number of promoters with a motif" and "number of motifs per promoter" is adapted from Figure 3. 
Table 3: Top 10 promoter-occurrence ranking list of the significantly enriched motifs and modules, within the auxin inducible promoters from $O$. sativa. Enrichment of cis-elements was determined by the parameter "number of promoters with a motif", whereas asterisks indicate an enriched with respect to “motif counts per promoter". Presented modules exhibit a variable, but maximal spacing of 100 bps between each embedded motif.

\begin{tabular}{|c|c|c|}
\hline combination & RANK & UP \\
\hline \multirow[t]{4}{*}{ single } & 1 & MRE2 \\
\hline & 2 & GRE* $^{*}$ \\
\hline & 3 & $\mathrm{TGA}^{*}$ \\
\hline & 4 & RY \\
\hline \multirow[t]{10}{*}{ bipartite } & 1 & GRE-MRE2 \\
\hline & 2 & TGA-MRE2* \\
\hline & 3 & MRE2-TGA* \\
\hline & 4 & AUX2-GRE \\
\hline & 5 & AUX1-MRE2 \\
\hline & 6 & MRE1-GRE \\
\hline & 7 & MRE1-TGA \\
\hline & 8 & GRE-MYC2 \\
\hline & 9 & AUX2-MYC2 \\
\hline & 10 & AUX1-GRE \\
\hline \multirow[t]{10}{*}{ tripartite } & 1 & MRE2-AUX2-GRE \\
\hline & 2 & AUX2-GRE-MRE2 \\
\hline & 3 & TGA-MRE2-AUX2 \\
\hline & 4 & MRE2-AUX2-TGA \\
\hline & 5 & MRE2-AC-AUX2 \\
\hline & 6 & MRE1-TGA-AUX2 \\
\hline & 7 & AUX2-GRE-MYC2 \\
\hline & 8 & MRE1-AUX2-GRE \\
\hline & 9 & AUX2-MYC2-GRE \\
\hline & 10 & TGA-AUX2-MYC2 \\
\hline
\end{tabular}

\section{Comparative analysis of ranked cis-elements and modules from Arabidopsis and} rice displays a conservation of specific cis-regulatory elements

In order to examine whether specific motifs or composite modules are conserved in auxin-induced promoters of Arabidopsis and rice, we performed a comparative analysis of the ranking positions of each cis-regulatory element. Unfortunately the available microarray data from the auxin-induction experiments from Arabidopsis and rice were differently designed so that comparable datasets had to be determined. The microarray data from the rice experiment were aimed at determining gene expression changes due 
to an 1 and 3 hour auxin treatment, wherefore both time-point transcript samples were pooled. Actually, analysing the Arabidopsis datasets only by comparing the 1 to 3 hour time-points results in a severe reduction of genes with altered auxin mediated expression, as it captured only the few genes that have much more dynamic expression changes at 3 hours compared to 1 hour post-treatment. As thus, the number of genes which were explicitly induced after $3 \mathrm{hrs}$ of auxin application was in the Arabidopsis dataset rather small, we decided to compare the dataset of the 1 hour up-regulated genes from Arabidopsis to that of the combined 1 to $3 \mathrm{hr}$ dataset from rice (Table 2; Table 3). Although we cannot exclude that rice plants react differently to the auxin application, a comparison of these datasets in fact resulted in the most concurrent list of enriched motifs in both species.

By this means it became apparent that only two single cis-elements were commonly enriched in the promoters of auxin up-regulated genes, - the GRE- and the RY-motif.

Concerning the bipartite motif combinations, all modules which were highly enriched in the Arabidopsis dataset were also present in the top 10 ranking list of rice. Most noticeable were the GRE-MRE2 module which ranked at $1^{\text {st }}$ position and the runner-up GRE-AUX1/2. Moreover the GRE-MRE1 and GRE-MYC2 as well as the AUX1/2MRE2 were further preferred modules as they ranked at various positions in the ranking lists of both angiosperm plant species. Thus, the 5 most enriched bipartite modules from Arabidopsis were also present in the top 10 ranking list of rice.

With respect to the top ranking triple cis-element combinations the GRE-AUX2-MRE2 and its related GRE-AUX2-MRE1 were the best conserved modules in Arabidopsis and rice. However the GRE-AUX2-MYC2 module was also highly abundant in the datasets of both species.

The most striking difference related to the TGA motif. It was found to be frequently abundant in the promoters of GmGH3 homologs (Figure 1) and occasionally enriched as single motif and in composite bi- and tripartite modules in the promoters of auxin upregulated genes from rice. In contrast to this, it was not overrepresented in the auxinresponsive promoters from Arabidopsis.

To sum up, the detailed comparison of the motif ranking lists of Arabidopsis and Rice (Table 2; Table 3) revealed that according to their ranking positions or occurrence, specific motifs and modules are conserved in the promoters of the auxin up-regulated 
genes from both species. These are mainly the GRE motif; all possible bipartite module combinations between GRE, AUX1/2 and MRE1/2 and the tripartite modules of GREAUX2-MRE1/2. The enrichment of the GRE and MRE motifs in association with the well-established auxin-responsive element (AuxRE) within the auxin inducible promoters from the tested monocot and dicot model plants strongly affirms their envisaged role in cooperatively regulating auxin responsive genes.

\section{Distinct motifs and composite cis-regulatory modules are enriched within promoters of auxin-related gene families}

To create a more detailed profile which auxin-responsive genes might actually be regulated by which specific type of motif or module we analysed the promoters of wellcharacterised auxin-regulated gene families such as the GH3s, SAURs and AUX/IAAs, which are described to be early auxin-responsive (Hagen and Guilfoyle, 2002, Liscum and Reed, 2002). However, all of them also include some late responsive members (Goda et al., 2004). In addition, also the family of the auxin-related ARF genes was studied, as an example of an auxin-related but largely constitutively expressed gene group (Ulmasov et al., 1999c). As the selected gene families have homologs or orthologs in Arabidopsis and rice, we intended to detect putatively conserved cisregulatory elements in the promoters of the specific auxin-regulated gene classes. Therefore, the available, respective promoter sequences from the genes from each gene family were compiled and tested for motif enrichment. In the following section an overview of the results from this gene class specific cis-element analysis is given (Figure 5). 


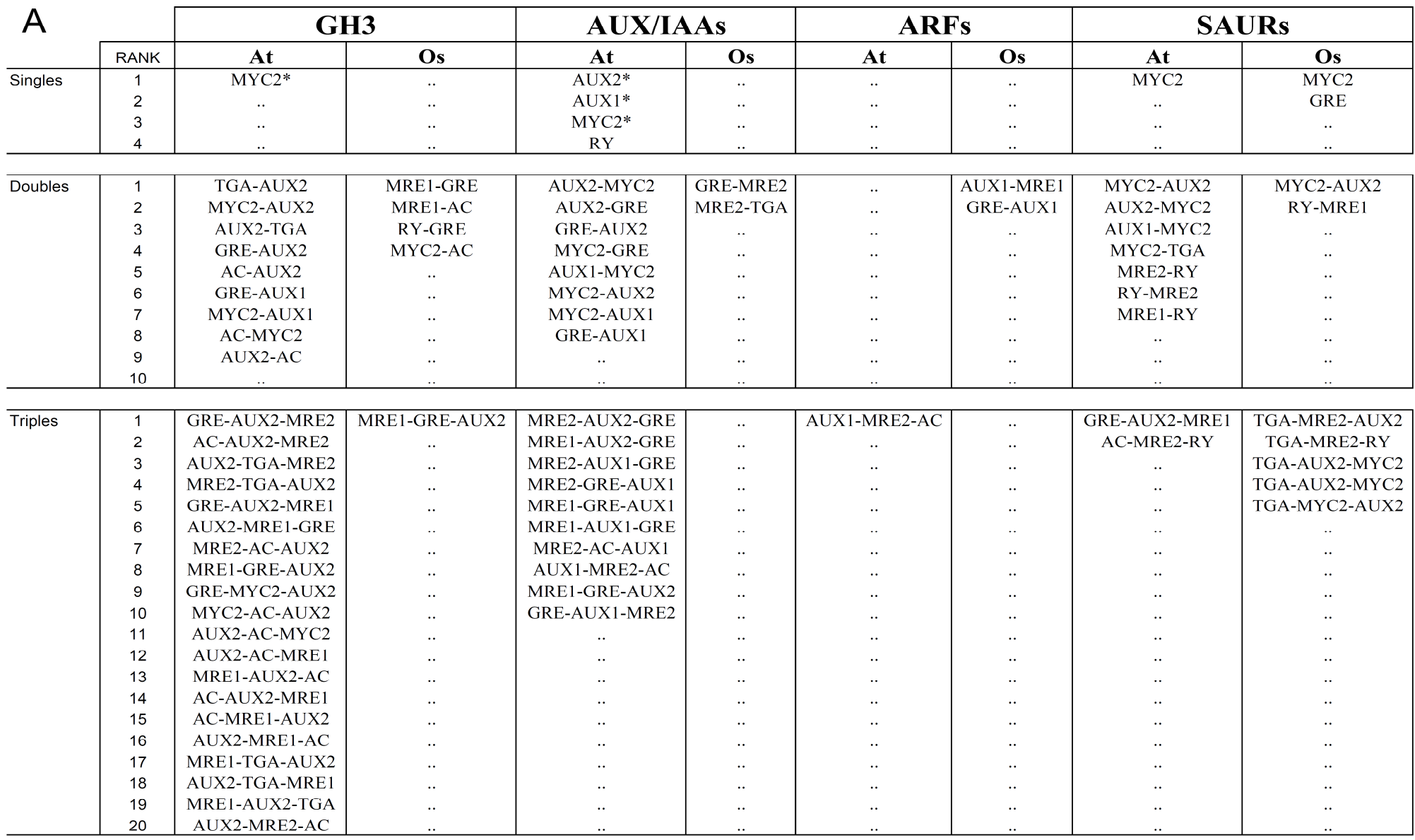

Figure 5: Promoter-occurrence ranking list of cis-elements and modules that are significantly enriched in specifc auxin responsive gene families from Arabidopsis and rice. Given is the significant motif and module enrichment or depletion regarding the "number of promoters with a motif", whereas asterisks indicate an enriched with respect to "motif counts per promoter". Presented modules exhibit a variable, but maximal spacing of 100 bps between each embedded motif. 
$G H 3 s$ - The promoters of the AtGH3 gene family are enriched for the single MYC2 motif, which also frequently occured in association with the AC cis-element in AtGH3 and $O s G H 3$ promoters. A more detailed evaluation of the bipartite modules revealed that the promoters of the $A t G H 3 s$ exhibit an enrichment of MYC2-AUX1/2 or BRE (GRE, TGA, AC)-AUX1/2 modules. In contrast to this the GRE and AC motifs frequently resided near a MRE1 motif in the $O s G H 3$ promoters. In the group of tripartite modules of the analysed TF-binding site classes, the module MRE1-GREAUX2 was enriched in both species. However, the frequently mentioned variant of it, the GRE-AUX2-MRE2 module was more preferred in Arabidopsis.

AUX/IAAs - The single AUX1/2, MYC2 and RY motifs were enriched in the promoters of the Arabidopsis AUX/IAA gene family, although the rice promoters did not share this. Concerning the bipartite modules the OsAUX/IAAs promoters exhibited an enrichment of GRE and TGA associations with MRE2, whereas the AtAUX/IAAs were strongly enriched for GRE-AUX1/2 and MYC2-AUX1/2 modules. The most prominent single GRE, MRE2 and AUX1/2 motifs in AtAUX/IAAs were present in different constellations in multiple GRE-AUX1/2-MRE2 composed tripartite modules in the AtAUX/IAA promoters.

$A R F s$ - The promoters of the AtARF and OSARF gene family displayed almost no enrichment for the cis-elements and modules, which were tested. Only the rice ARF promoters showed a higher abundance of the GRE-AUX1 and the MRE1-AUX1 bipartite modules and the Arabidopsis ARFs for the AC-MRE2-AUX1 tripartite module.

SAURs - In Arabidopsis and rice, the promoters of the SAUR gene family were significantly enriched for the single MYC2 motif. This conservation also persisted in the modules in which a combination of the MYC2 and AUX1/2 occured in almost each enriched bipartite module of both species. Only the RY-MRE1 module which was also conserved in both species differed in this respect. In rice, the MYC2 motif also remained relevant in tripartite modules, in a TGA motif extended version of the MYC2AUX2. Nevertheless the most abundant tripartite modules in both species did not 
contain a MYC2 motif as these were the GRE-AUX2-MRE1 module in AtSAUR and the TGA-MRE2-AUX2 module in OSSAUR promoters. Finally, it has to be mentioned that OSSAUR promoters were in general enriched for tripartite modules containing a TGA motif.

All in all, it can be concluded that the homologous auxin-regulated gene families from Arabidopsis and rice showed only little overlap for specific cis-regulatory elements (Figure 5). However some distinct modules can be highlighted which might play a role in species- and family-specific induction kinetics. Noticeable in this respect is the observation that the primarily early auxin-responsive gene families such as the AtGH3s and AtAuxIAAs exhibit an enrichment of the GRE-AUX1/2 module, which is missing in the promoters of the mainly constitutively expressed AtARF genes. Moreover it has to be pointed out that the families of the Arabidopsis and rice SAUR genes show a strong enrichment for the single MYC2 and MYC2-AUX1/2 modules.

Surprisingly, neither the single AUX1/2, nor the single GRE-motif is frequently enriched in the analysed auxin-regulated gene classes, although this had been shown for the set of all auxin-responsive promoters from Arabidopsis (Figure 3; Table 2). These observations suggest a putative dependency of specific cis-element combinations in the regulation of auxin-responsive gene classes.

Mutations in the GRE cis-element within a GRE-AuxRE module of the Arabidopsis GH3.3 promoter lead to a significant reduction of its auxin-responsiveness

To validate the bioinformatic based assumption that GRE and AuxRE cis-elements cooperate in auxin mediated transcription, we inspected the promoters of the early auxinresponsive Arabidopsis GH3 genes for the presence of GREs. AtGH3.3 is a close homolog of the well- characterised soybean GmGH3 and harbours several GREs in its promoter in close vicinity to AuxRE- and MRE cis-elements and to the transcriptional start site (TSS) (Figure 6A; Figure 1). In fact, some of them also form the GREAuxRE-MRE tripartite-module, which has been shown to be frequently enriched in $A t G H 3$ gene promoters (Figure 6A).

In order to analyse the effect of the GRE motif on auxin mediated transcription, a short synthetic AtGH3.3 derived promoter region containing one GRE, one consensus AuxRE 
and an adjacent inversely oriented AuxRE cis-element, was constructed, which we

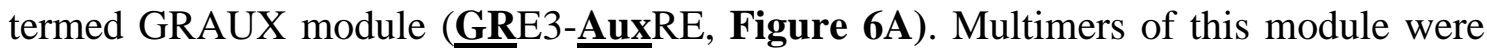
fused to a minimal promoter and a GUS reporter gene, allowing expression analysis via transient protoplast transfection assays. Whereas a single GRAUX-module construct exhibited a slightly, but not significantly higher reporter gene expression in the presence of low exogenous auxin concentrations, multimerisation strongly enhanced auxin responsiveness (Figure 6B). Notably all constructs showed a similar background expression under non-inductive conditions (Figure 6B).

To assign the influence of the GRE motif within the GRAUX-module, cis-element specific mutational derivatives of the 4-times multimerised construct were analysed. By this means, it could be demonstrated that mutations in the core sequence of the GREmotif resulted in a $\sim 54 \%$ reduction of inducibility, while mutations in the AuxREs alone or in combination with a mutated GRE, lead to complete auxin insensitivity (Figure 6C).

In order to validate the results obtained from the synthetic GRAUX-module promoter and to define the impact of the GREs in the genuine AtGH3.3 promoter context, a corresponding full-length promoter:GUS construct was generated. Applying site directed mutagenesis, a promoter construct was generated that lacked all 4 GREs located near the TSS (Figure 6D). Whereas the native AtGH3.3 promoter-construct revealed a strong and significant auxin mediated expression, the mutated promoterconstruct displayed a $51 \%$ reduction in its auxin-triggered induction (Figure 6D). As we have demonstrated that auxin-inducible promoters are in general enriched for GREAuxRE (GRAUX) modules they are certainly potential quantitative elements in auxinregulated promoters.

\section{Discussion}

In this work, we have presented a genome wide cis-element analysis of bZIP- (BRE), MYB/MYC- (MRE/MYC2) and ARF-TF (AuxRE/RY) related binding sites in auxinresponsive promoters from Arabidopsis and rice. We could demonstrate that specific cis-elements and composite modules, which encompass typical binding sites for these 
A

\begin{tabular}{|c|}
\hline 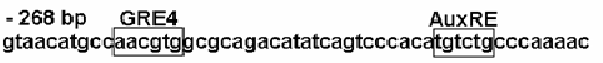 \\
\hline 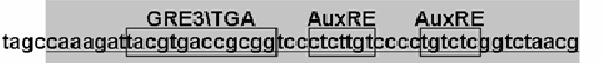 \\
\hline $\begin{array}{l}\text { MREEMRE } \\
\text { ataacaaaccgagcccactttatgtcgacgtggaattggctgacg aAMRE }\end{array}$ \\
\hline ctccttcttgccactataaatacaaccccatactcgtcgagtttcaatatctcctc \\
\hline atcatcaaacacaaagtctaatattatcacttacaaataccatttaatcccATG \\
\hline GRAUX-module promoter construct \\
\hline GRE AuxRE AuxRE \\
\hline
\end{tabular}

C

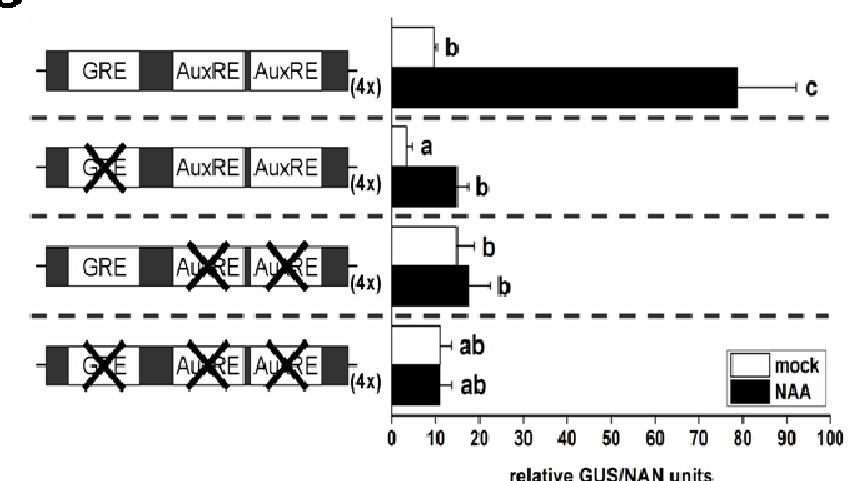

B

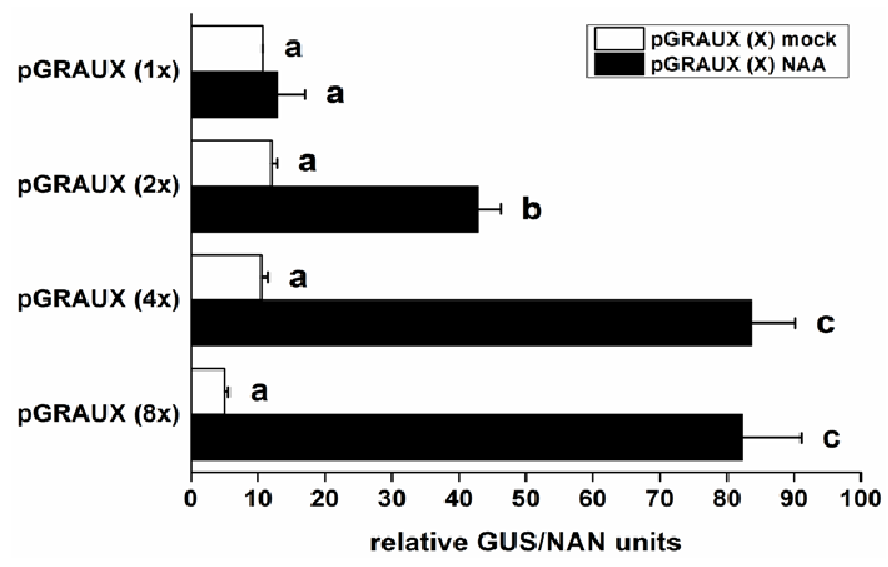

D

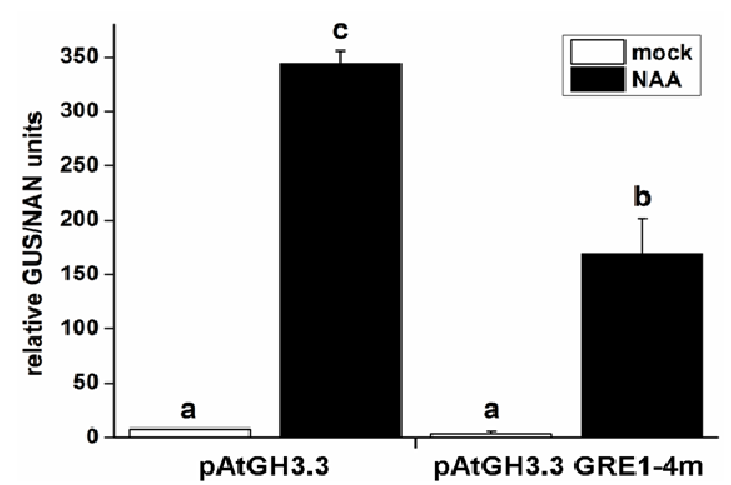

Figure 6: Molecular characterisation of the GRAUX-module. A) GRE, MRE and AuxRE cis-elements within the -268bp AtGH3.3 promoter region. White boxes indicate the positions of the GRE-, MRE- and AuxRE motifs within the AtGH3.3 promoter sequence close to the transcriptional start site (TTS). The present GREs are serially numbered (1-4). The grey highlighted sequence represents the AtGH3.3 promoter-region used as synthetic auxin-responsive GRAUX-module promoter construct. B) Expression profile of synthetic pGRAUX:GUS reporter constructs. Numbers of multimers are indicated. C) Auxin inducibility of the pAtGH3.3 derived pGRAUX(4x)-module reporter construct and its mutational derivates. A schematic view of the transfected reporter constructs is given. Mutated cis-elements are indicated by X. D) Auxin-responsiveness of the AtGH3.3 promoter:GUS construct and a GRE motif mutated version. If not stated elsewhere white coloured bars generally represent transfected, mock treated (DMSO) and black coloured bars NAA treated $(0.25 \mu \mathrm{M}, 16 \mathrm{~h})$ samples. All presented results were obtained from transient protoplast transfection assays. Given are the mean GUS/NAN values $( \pm$ SD) from 3 independent experiments. Different letters ( $\mathrm{p} \leq 0,05$; one-way ANOVA followed by Fisher post-hoc test) or asterisks (Students T-Test; $\mathrm{p} \leq 0,05=* ; \mathrm{p} \leq 0,01=* * ; \mathrm{p} \leq 0,001=* * *)$ denote significant differences between the used constructs and treatments. 
TFs, are in general significantly enriched in auxin inducible promoters in both angiosperm plant species tested. Most prominent and concurrent in this respect, is the enrichment of the single GRE, MRE2 and AUX1/2 motifs as well as their related biand tripartite-module organisations. Moreover a substantial enrichment of RY and MYC2 cis-elements was observed. The evolutionary conservation of these cis-acting elements in the analysed monocot and dicot model plants, that exhibit considerable differences in their species specific genome structure in terms of the GC content, affirms that they might be part of a common regulatory mechanism in auxin-responsive transcription. Gene family specific cis-element analysis revealed that especially promoters from early auxin-responsive gene classes are enriched for GRE-AuxRE modules. In fact, we can demonstrate that mutations in the GRE motifs within the native early auxin-responsive $A t G H 3.3$ promoter result in a severe reduction of its auxin triggered inducibility.

\section{Enrichment is a relevant criterion for predicting the function of a promoter cis- element however the importance of some cis-elements might be underrated}

In the presented cis-element analysis we focused on the enrichment and clustering of specific cis-elements in auxin-responsive promoters to predict which motif is important in this functional context. However, the validity of this cis-element analysis is based on and limited by the main assumption that sets of putative target genes, which are regulated by the same type of $\mathrm{TF}$, should contain its cognate binding sites in all or in a relevant proportion of their promoters. This principle makes it reasonable to propose single genes or whole co-regulated gene groups as $\mathrm{TF}$ targets by the presence or enrichment of its according binding site. However this is not mandatory. For example it has been reported that some TFs co-operatively regulate their target genes by interacting with other proteins that have DNA-binding properties. These adaptors mediate the association with the DNA and thereby recruit specific TFs independent on their own promoter binding and thus independent on the TFs' related cis-element (Alonso et al., 2009a).

Besides this, it is conceivable that specific cis-elements, which frequently occur throughout the genome, might not be considered relevant as they are not significantly enriched in specific promoter subsets. This indicates that the role of some motifs and 
their cognate TFs might thus be underrated with this approach. An example for this is the AuxRE motif itself. It is well-documented that it is present in most, but not all auxin-responsive promoters, and in those in which it is present, it does not always occur in overabundant numbers (Pufky et al., 2003, Nemhauser et al., 2004). In agreement with this we could not find an enrichment of the single AuxRE in the auxin responsive promoters from rice or any auxin regulated gene class we have tested, however it is enriched in the promoter set of all auxin inducible genes from Arabidopsis. Anyhow, it is known as the most important qualitative element which confers auxin sensitivity to auxin responsive promoters (Ulmasov et al., 1995).

Nevertheless, it can conversely still be assumed that specific motifs that are found to be significantly enriched are potential binding sites for essential TFs regulating the corresponding promoters.

Concerning the Motif Mapper cis-element analysis software used in this study, the applied randomization algorithm proved to be straightforward and effective for accessing the significance of cis-elements and composite cis-element modules of any unclustered gene group. In contrast to other more complex cis-regulatory module scanners that require clusters of tightly co-expressed genes and/or sets of orthologous genes (Van Loo and Marynen, 2009, Wrzodek et al., 2010), our algorithm performed well in noisy, unclustered datasets, as it just counts all defined motifs and/or combinations without seeking for optimal concurrent motifs and/or combinations to explain a given dataset clustering. The program in fact, applies the determined number of distinct motifs and modules in the randomized dataset and the finite number of ciselements in the experimental dataset to immediately validate enrichment of motifs or modules. Therefore, any association of genes can be chosen and analyzed (e.g. gene families, GO annotations, etc.) and tested for $c i s$-element enrichment and/or depletion. Due to the simplicity of the algorithm, it also requires less bioinformatic knowledge compared to the majority of other CRM programs, making it more accessible to nonexpert user without compromising the analysis quality. The user only needs to provide a complete genomic set of promoters, of which several are now available, a specific promoter subset of interest and a list of cis-elements and/or combinations which should be tested. 
BRE and MRE cis-elements are potential quantitative coupling elements for AuxREs

As mentioned earlier diverse BRE, MRE/MYC2 and AuxRE related motifs and composite modules were found to be significantly enriched in the auxin-responsive promoters from Arabidopsis and rice (Figure 2; Figure 3; Table 2; Table 3) and particularly in early auxin-responsive gene classes, like the GH3s, AUX/IAAs and SAURs, whereas they were not enriched in the class of the largely constitutively expressed $A R F$ genes (Figure 5). The most outstanding in this respect are the single GRE- and RY-motifs which are strongly enriched in the promoters from all auxin upregulated genes from Arabidopsis and rice, followed by the AUX1 and MYC2 which are enriched in the Arabidopsis auxin up-regulated promoters and MRE2 which is most frequent in those from rice. However, the relevance of some of these motifs become more pronounced and of others only concrete in association with additional motifs. Particularly the MRE motifs are frequently enriched in a modular structure with GREs or AuxREs in auxin-inducible promoters from both analysed species (Figure 2; Figure 3; Table 2; Table 3). Thereby the MRE2 seems to be more preferred compared to the MRE1, whereas this could be partially explained by the fact that the MRE1 motif is slightly more rare than the MRE2. The enrichment of the MRE-AuxRE associations suggests that MREs are a relevant integration platform for MYB-TF activities which is also supported by experimental data provided by Shin et al. (2007). Consistent with our observation that MREs frequently reside near AuxREs the authors were able to show that AtMYB77 effectively interacts with AtARF7 to synergistically promote target gene expression. All in all, this indicates that MREs function as coupling elements for AuxRE mediated transcription, in both, monocot and dicot plant species.

Similar observations can be made for the BRE motifs. They also occur in high frequency in context with adjacent AuxRE elements. The GRE-AuxRE module is in this respect the by far most predominant combination in all auxin-inducible promoters and specifically in those of early auxin-responsive genes from both species. Although, it is present in only $6 \%$ of all Arabidopsis and $2 \%$ of the rice promoters, it clusters in the auxin-responsive promoters with $41 \%$ among the up-regulated and $21 \%$ of the downregulated genes in Arabidopsis, and with around $32 \%$ among the up- and downregulated gene promoters from rice. Referring to these percentages, it has to be 
considered that in general more genes are rather up- than down-regulated by the auxin treatment and that these frequencies are much higher than the expected random distribution which is around $6 \%$.

These data are supported by publications that report that G-box like cis-elements are coupling elements of the AuxREs in the early auxin-responsive promoters of the soybean $G H 3$ and $A U X 28$ genes and that they synergistically promote their transcription (Nagao et al., 1993, Hong et al., 1995, Ulmasov et al., 1995, Liu et al., 1997a). In line with this, the functional relevance of the GRE as quantitative coupling element was further confirmed experimentally in transient protoplast transfection assays, by analysing the synthetic GRAUX module or the native AtGH3.3 promoter (Figure 6A D). Furthermore, it was demonstrated that a tobacco $N t G H 3$ gene harbours multiple GRE-AuxRE repetitions in its promoter and that NtBZI-1 binds to them. This TF promotes the expression of the $N t G H 3$ gene in an auxin dependent manner, whereas a dominant-negative derivative of it diminishes the expression (Heinekamp et al., 2004). As homologs of this tobacco bZIP have been characterized to be involved in reprogramming transcription in response to low energy stress (Baena-Gonzalez et al., 2007, Dietrich et al., 2011), these data propose a model in which information about the cellular energy homeostasis is integrated into auxin-specific gene expression.

Taken together, our observations along with these, suggest that GRE-AuxRE modules are conserved quantitative cis-elements in monocot and dicot plant species that at least contribute to the expression of some early auxin-responsive genes in multiple plant species.

Besides the described GRE-AuxRE and MRE-AuxRE modules, GRE-AuxRE-MRE tripartite modules are substantially enriched in the promoters of auxin inducible genes from Arabidopsis and rice (Table 2; Table 3) and in those from the Arabidopsis early auxin-regulated gene classes (Figure 5). This establishes the possibility of complex, combinatorial transcriptional regulation. However, as genes are characterized by specific differences in their expression patterns, differences in the cis-element structure can be envisaged. MYB- and bZIP-TFs are known to be involved in regulating diverse developmental and stress-related processes in plants (Stracke et al., 2001, BaenaGonzalez et al., 2007, Alonso et al., 2009a, Dietrich et al., 2011). They might therefore 
singularly or co-operatively integrate changing developmental or stress-related inputs in auxin mediated responses.

\section{RY and MYC2 binding sites are strongly enriched cis-elements in auxin-responsive promoters with yet undefined relevance}

Based on the cis-element analysis of auxin-responsive promoters from Arabidopsis and rice we moreover detected a significant enrichment of RY and MYC2 cis-elements in auxin inducible promoters (Figure 2; Figure 3). Concerning the single MYC2 motif we observed a strong enrichment in the auxin inducible promoters from Arabidopsis and more precisely, also as single and in association with AuxREs in the promoters of the auxin-responsive SAUR gene families from Arabidopsis and rice (Figure 5). Consistent with that, Pufky and co-workers (Pufky et al., 2003) revealed in an unbiased approach that MYC2 motifs were more frequently found in small clusters of auxin inducible promoters from Arabidopsis compared to the expected genomic frequency and Nemhauser and co-workers (Nemhauser et al., 2004) that MYC2 cis-elements are overrepresented in promoters of auxin and brassinosteroid responsive genes. Even though these findings suggest that the MYC2 motif might be involved in modulating the expression of auxin-responsive genes it is yet unclear which function it exerts. Quite recently it was reported that the MYC2-motif is enriched in promoters from diurnally regulated genes and that it is in fact sufficient to confer the observed circadian expression pattern in vivo (Michael et al., 2008). As many growth related processes, which are mainly mediated by auxin, are also interconnected with the circadian clock (Robertson et al., 2009), the MYC2-element might provide a molecular link between these systems.

Regarding the single RY motif we have demonstrated that it is enriched in auxininducible promoters from Arabidopsis and rice, even though it was only present in 3\% of all Arabidopsis and 7\% of all rice promoters. The RY motif or Sph/Ry-box (Suzuki et al., 1997) was reported to be involved in abscisic acid (ABA) signalling (Finkelstein and Gibson, 2002). It is known to be bound by members of the B3-type TFs superfamily, which also includes ARF TFs. One of these members, which directly binds the RY cis-element is the $\mathrm{ABI} 3 \mathrm{TF}$, which contains three basic domains, originally designated B1, B2 and B3 (Suzuki et al., 1997). Whereas, the B3 domain was 
demonstrated to be necessary for RY binding (Suzuki et al., 1997), the B2 domain appears to be responsible for recruiting bZIP TFs (Hill et al., 1996, Ezcurra et al., 2000, Nakamura et al., 2001). An example of such interplay of ABI3 and bZIP TFs was described by Alonso and co-workers (Alonso et al., 2009a), which reported that these TFs synergistically promote the expression of seed maturation genes, including seed storage and dehydration-responsive genes. Based on these observations it is conceivable that the RY motif might be involved in altering auxin-responsive gene expression in response to dehydration or other $\mathrm{ABA}$ regulated processes, especially during seed development.

\section{Conclusion}

In order to adjust auxin controlled growth-related and developmental processes in plants in response to the diversity of changing environmental cues, it can be strongly assumed that besides the well-characterised qualitative AuxRE-elements, additional quantitative motifs are needed to accordingly alter the expression of auxin-responsive genes. Indeed, modular organisations of GRE- and MRE-motifs in association with AuxREs are highly enriched and evolutionary conserved and appear to synergistically contribute to auxininducible expression. The finding that RY and MYC2 motifs are highly enriched in auxin-responsive promoters, further increases the combinatorial, integrative opportunities in auxin mediated transcription. Furthermore, these data demonstrate the potential of bioinformatic approaches to establish working hypotheses on novel ciselements and to design experiments to characterize their predicted functions.

\section{Material and Methods}

\section{Phylogenetic and cis-element analysis of $G H 3$ promoters}

GH3 promoter sequences were sourced from Plant Genome Database (see references). All available plant genomes were queried and scanned for putative GmGH3 (NCIB: accession X60033; (Hagen et al., 1991)) homologs using tBLASTX. Sequence matches with BLAST scores $\leq 0.00001$ were retained to identify putative orthologs. From plant genomes with sufficient sequence coverage, -1000 bp promoter sequences were 
extracted. AuxREs, BREs and MREs were mapped and illustrated using TOUCAN 2 (Aerts et al., 2005). The GH3 protein sequences which were obtained from their corresponding cDNA sequences were used for subsequent phylogenetic analysis applying the ClustalW2 software (see references). The phylogenetic tree was designed with TreeView software (Page, 2002).

\section{Microarray Analysis}

Auxin-responsive genes from A. thaliana and O. sativa were identified by analysing the microarray data from auxin treatment experiments. For Arabidopsis, wt seedling experiment with an induction time course of $1 \mu \mathrm{M}$ IAA for $30 \mathrm{~min}, 1 \mathrm{~h}$ and $3 \mathrm{~h}$ (TAIR ExpressionSet: 1007965859) was used and for rice (Oryza sativa variety IR64) a wt seedling experiment with an auxin induction from 1 to $3 \mathrm{~h}$ (NCBI Gene Expression Omnibus GSE5167). The according data files were imported into GenSpring GX 7.3.1 (Agilent) and the datasets were adjusted to remove the background of optical noise using the GC-RMA software (Han et al., 2004) and finally normalized by quantile normalization. For further analysis only 2-fold up- or down-regulated genes after auxin treatment compared to controls were used.

\section{Promoter sequence retrieval}

The Arabidopsis promoter dataset used to create the control randomized dataset was obtained from Arabidopsis TAIR 9 release (Lamesch et al., 2010) and cleaned from promoters of plastidial and alternative transcribed genes. For cis-element mapping promoters from A. thaliana and $O$. sativa were extracted from GenBank files provided by NCBI (see references). Arabidopsis promoters were extracted according to their TAIR 9 annotations or rice promoters using the GenBank extraction script aGBSQL of the Motif Mapper for Python v1.2 software (see references), respectively. For all promoters $-1000 \mathrm{bp}$ upstream sequences were extracted beginning at the most 5' annotation point for each gene [option TSS or ATG], excluding the base pair at the 5' position. The $O$. sativa promoter dataset was extracted from NCBI, analogously. 


\section{Gene Descriptions}

Functional gene descriptions for $O$. sativa were taken from RAP3_locus GFF3 file provided by Rice Annotation Project Database (see references). Redundant entries, due to alternative transcripts were manually removed. Functional gene descriptions for $A$. thaliana were taken from TAIR9_functional_descriptions dump file. This list was cross referenced using the TAIR Gene Descriptions web based query tool. Redundant entries due to alternative transcripts were manually removed.

\section{Real Randomization algorithm}

The Cluster Analysis Real Randomization algorithm (Motif Mapper v5.1.1.39) was written to facilitate sequence extraction from input FASTA sequence files and to define significant motif enrichments compared to a created randomized dataset. Therefore, initially a list of given motifs is entered manually or per text file and they are mapped for the entire dataset. Motifs and composite modules can be analyzed on Watson strands only or include their Crick strand in the calculation ("auto-antisense" or "dyadic(auto)", respectively). Lists of genes are read automatically from any number of text files present in any number of folders; such lists are automatically extracted from any tab-delimited text file that has a common header. Thereafter, the algorithm extracts random gene sets equal in size to the number of sequence matches actually found in the FASTA database and records the match values. This is then repeated for the number of iterations the user defined to build the background population statistics. The actual gene set is gathered and a Z-score calculation is made based on the randomized background population distribution. The results from the Z-score calculation are returned to the user per input gene set with an option to also have the randomization values as well. To calculate the false-positive error rate; a script was written to reiteratively call the Cluster Analysis Real Randomization algorithm and put the raw data to text files from each pass for calculating the false-positive error rates.

\section{Plant cultivation, protoplast transformation and GUS-Assays}

4 to 5 weeks old Arabidopsis Col-0 plants (grown on soil under long day conditions at $23^{\circ} \mathrm{C}$ and a relative humidity of $60 \%$ ) were used for protoplast preparation. Protoplast 
isolation and transformation was performed according to Ehlert and co-workers (Ehlert et al., 2006). For promoter activation assays $14 \mu \mathrm{g}$ of the promoter:GUS reporter plasmid was co-transfected with $3 \mu \mathrm{g}$ of a normalization plasmid (Pro35S:NAN). After transformation the protoplasts were incubated for $16 \mathrm{~h}$ in an incubation buffer supplemented with varying concentrations of NAA (Sigma-Aldrich, Germany) or DMSO (mock). GUS and NAN enzyme assays were performed according to (Kirby and Kavanagh, 2002). The ratio of GUS to NAN activities are calculated as relative GUS/NAN values. Statistical analysis was performed using the software OriginPro 8.1G (OriginLabs).

\section{Molecular biological techniques}

Standard DNA techniques have been described by Sambrook and co-workers (Sambrook, 1989). DNA mutagenesis was performed applying the Quick Change site directed mutagenesis kit (Stratagene, Amsterdam, Netherlands) following the manufacturer`s manual. DNA sequence analysis was performed using an ABI310 sequencer (Applied Biosystems, Darmstadt, Germany) with an ABI PRISM BigDye terminator cycle sequencing reaction kit (Applied Biosystems, Darmstadt, Germany). Plasmid construction has been described in Weiste et al., (Chapter 2).

\section{List of Abbreviations}

TFs - Transcription Factors, BRE - bZIP Response Element, GRE - G-box related Element, TGA - TGA Response Element, AC - ACTCAT motif of S1-group bZIP-TFs, MRE - MYB Response Element, AuxRE - Auxin Response Element, ARFs - Auxin Response Factors, AUX/IAAs - AUX/IAA repressor proteins, WT - wildtype.

\section{Authors' contributions}

$\mathrm{CW}$ and $\mathrm{KB}$ wrote the manuscript and interpreted the results. $\mathrm{KB}$ wrote the algorithm and $\mathrm{KB}$ and DW performed the cis-element analysis, JK did the microarray analysis, CW generated reporter constructs, performed and analysed transient protoplast 
transfection experiments. CW, KB and WDL designed the study. WDL and KH revised the manuscript.

\section{References}

Abe H, Yamaguchi-Shinozaki K, Urao T, Iwasaki T, Hosokawa D, Shinozaki K. 1997. Role of arabidopsis MYC and MYB homologs in drought- and abscisic acid-regulated gene expression. Plant Cell, 9: 1859 .

Abel S, Ballas N, Wong LM, Theologis A. 1996. DNA elements responsive to auxin. Bioessays, 18: 647.

Aerts S, Van Loo P, Thijs G, Mayer H, de Martin R, Moreau Y, De Moor B. 2005. TOUCAN 2: the all-inclusive open source workbench for regulatory sequence analysis. Nucleic Acids Res, 33: W393.

Alonso R, Onate-Sanchez L, Weltmeier F, Ehlert A, Diaz I, Dietrich K, Vicente-Carbajosa J, Droge-Laser W. 2009. A pivotal role of the basic leucine zipper transcription factor bZIP53 in the regulation of Arabidopsis seed maturation gene expression based on heterodimerization and protein complex formation. Plant Cell, 21: 1747.

Baena-Gonzalez E, Rolland F, Thevelein JM, Sheen J. 2007. A central integrator of transcription networks in plant stress and energy signalling. Nature, 448: 938.

Ballas N, Wong LM, Theologis A. 1993. Identification of the auxin-responsive element, AuxRE, in the primary indoleacetic acid-inducible gene, PS-IAA4/5, of pea (Pisum sativum). J Mol Biol, 233: 580.

Bennett T, Scheres B. 2010. Root development-two meristems for the price of one? Curr Top Dev Biol, 91: 67.

Berendzen KW, Stuber K, Harter K, Wanke D. 2006. Cis-motifs upstream of the transcription and translation initiation sites are effectively revealed by their positional disequilibrium in eukaryote genomes using frequency distribution curves. BMC Bioinformatics, 7: 522.

Brown CD, Johnson DS, Sidow A. 2007. Functional architecture and evolution of transcriptional elements that drive gene coexpression. Science, 317: 1557.

Chapman EJ, Estelle M. 2009. Mechanism of auxin-regulated gene expression in plants. Апnи Rev Genet, 43: 265.

Chen W, Provart NJ, Glazebrook J, Katagiri F, Chang HS, Eulgem T, Mauch F, Luan S, Zou G, Whitham SA, Budworth PR, Tao Y, Xie Z, Chen X, Lam S, Kreps JA, Harper JF, Si-Ammour A, Mauch-Mani B, Heinlein M, Kobayashi K, Hohn T, Dangl JL, Wang X, Zhu T. 2002. Expression profile matrix of Arabidopsis transcription factor genes suggests their putative functions in response to environmental stresses. Plant Cell, 14: 559.

Cornish-Bowden A. 1985. Nomenclature for incompletely specified bases in nucleic acid sequences: recommendations 1984. Nucleic Acids Res, 13: 3021.

Dharmasiri N, Dharmasiri S, Estelle M. 2005. The F-box protein TIR1 is an auxin receptor. Nature, 435: 441.

Dickinson CD, Evans RP, Nielsen NC. 1988. RY repeats are conserved in the 5'-flanking regions of legume seed-protein genes. Nucleic Acids Res, 16: 371.

Dietrich K WF, Ehlert A, Weiste C, Stahl M, Harter K, Dröge-Laser W. 2011. Heterodimers of the Arabidopsis Transcription Factors bZIP1 and bZIP53 are Reprogramming Amino Acid Metabolism during Low Energy Stress. Plant Cell, in press.

Ehlert A, Weltmeier F, Wang X, Mayer CS, Smeekens S, Vicente-Carbajosa J, Droge-Laser W. 2006. Two-hybrid protein-protein interaction analysis in Arabidopsis protoplasts: establishment of a heterodimerization map of group C and group S bZIP transcription factors. Plant J, 46: 890.

Ezcurra I, Wycliffe P, Nehlin L, Ellerstrom M, Rask L. 2000. Transactivation of the Brassica napus napin promoter by $\mathrm{ABI} 3$ requires interaction of the conserved $\mathrm{B} 2$ and $\mathrm{B} 3$ domains of $\mathrm{ABI} 3$ with different cis-elements: B2 mediates activation through an ABRE, whereas B3 interacts with an RY/G-box. Plant J, 24: 57. 
Finkelstein RR, Gibson SI. 2002. ABA and sugar interactions regulating development: cross-talk or voices in a crowd? Curr Opin Plant Biol, 5: 26.

Goda H, Sawa S, Asami T, Fujioka S, Shimada Y, Yoshida S. 2004. Comprehensive comparison of auxin-regulated and brassinosteroid-regulated genes in Arabidopsis. Plant Physiol, 134: 1555.

Gray WM, Kepinski S, Rouse D, Leyser O, Estelle M. 2001. Auxin regulates SCF(TIR1)-dependent degradation of AUX/IAA proteins. Nature, 414: 271.

Guilfoyle T, Hagen G, Ulmasov T, Murfett J. 1998a. How does auxin turn on genes? Plant Physiol, 118: 341 .

Guilfoyle TJ, Ulmasov T, Hagen G. 1998b. The ARF family of transcription factors and their role in plant hormone-responsive transcription. Cell Mol Life Sci, 54: 619.

Hagen G, Guilfoyle T. 2002. Auxin-responsive gene expression: genes, promoters and regulatory factors. Plant Mol Biol, 49: 373.

Hagen G, Martin G, Li Y, Guilfoyle TJ. 1991. Auxin-induced expression of the soybean GH3 promoter in transgenic tobacco plants. Plant Mol Biol, 17: 567.

Han ES, Wu Y, McCarter R, Nelson JF, Richardson A, Hilsenbeck SG. 2004. Reproducibility, sources of variability, pooling, and sample size: important considerations for the design of highdensity oligonucleotide array experiments. J Gerontol A Biol Sci Med Sci, 59: 306.

Heinekamp T, Strathmann A, Kuhlmann M, Froissard M, Muller A, Perrot-Rechenmann C, Droge-Laser W. 2004. The tobacco bZIP transcription factor BZI-1 binds the GH3 promoter in vivo and modulates auxin-induced transcription. Plant J, 38: 298.

Hill A, Nantel A, Rock CD, Quatrano RS. 1996. A conserved domain of the viviparous-1 gene product enhances the DNA binding activity of the bZIP protein EmBP-1 and other transcription factors. $J$ Biol Chem, 271: 3366.

Hong JC, Cheong YH, Nagao RT, Bahk JD, Key JL, Cho MJ. 1995. Isolation of two soybean G-box binding factors which interact with a G-box sequence of an auxin-responsive gene. Plant J, 8: 199.

Jain M, Khurana JP. 2009. Transcript profiling reveals diverse roles of auxin-responsive genes during reproductive development and abiotic stress in rice. FEBS J, 276: 3148.

Kepinski S, Leyser O. 2005. The Arabidopsis F-box protein TIR1 is an auxin receptor. Nature, 435: 446.

Kirby J, Kavanagh TA. 2002. NAN fusions: a synthetic sialidase reporter gene as a sensitive and versatile partner for GUS. Plant J, 32: 391.

Lamesch P, Dreher K, Swarbreck D, Sasidharan R, Reiser L, Huala E. 2010. Using the Arabidopsis information resource (TAIR) to find information about Arabidopsis genes. Curr Protoc Bioinformatics, Chapter 1: Unit1 11.

Leyser O. 2005. The fall and rise of apical dominance. Curr Opin Genet Dev, 15: 468.

Liscum E, Reed JW. 2002. Genetics of Aux/IAA and ARF action in plant growth and development. Plant Mol Biol, 49: 387.

Liu ZB, Hagen G, Guilfoyle TJ. 1997. A G-Box-Binding Protein from Soybean Binds to the E1 AuxinResponse Element in the Soybean GH3 Promoter and Contains a Proline-Rich Repression Domain. Plant Physiol, 115: 397.

Liu ZB, Ulmasov T, Shi X, Hagen G, Guilfoyle TJ. 1994. Soybean GH3 promoter contains multiple auxin-inducible elements. Plant Cell, 6: 645.

Martin C, Paz-Ares J. 1997. MYB transcription factors in plants. Trends Genet, 13: 67.

Menkens AE, Cashmore AR. 1994. Isolation and characterization of a fourth Arabidopsis thaliana Gbox-binding factor, which has similarities to Fos oncoprotein. Proc Natl Acad Sci U S A, 91: 2522.

Michael TP, Breton G, Hazen SP, Priest H, Mockler TC, Kay SA, Chory J. 2008. A morning-specific phytohormone gene expression program underlying rhythmic plant growth. PLoS Biol, 6: e225.

Moller B, Weijers D. 2009. Auxin control of embryo patterning. Cold Spring Harb Perspect Biol, 1: a001545.

MotifMapper software [http://www.mnf.uni-tuebingen.de/fachbereiche/zentren/zmbp/plant-physiology /research-groups/harter/berendzen/motif-mapper-for-python.html

Muday GK. 2001. Auxins and tropisms. J Plant Growth Regul, 20: 226. 
Nagao RT, Goekjian VH, Hong JC, Key JL. 1993. Identification of protein-binding DNA sequences in an auxin-regulated gene of soybean. Plant Mol Biol, 21: 1147.

Nakamura S, Lynch TJ, Finkelstein RR. 2001. Physical interactions between ABA response loci of Arabidopsis. Plant J, 26: 627.

NCBI, National Center for Biotechnology Information [http://www.ncbi.nlm.nih.gov/]

Nemhauser JL, Mockler TC, Chory J. 2004. Interdependency of brassinosteroid and auxin signaling in Arabidopsis. PLoS Biol, 2: E258.

Page RD. 2002. Visualizing phylogenetic trees using TreeView. Curr Protoc Bioinformatics, Chapter 6: Unit 62.

Phylogenetic analysis tool, Clustal W [http://www.ebi.ac.uk/Tools/clustalw2/index.html]

Plant Genome Database [http://www.plantgdb.org/]

Pufky J, Qiu Y, Rao MV, Hurban P, Jones AM. 2003. The auxin-induced transcriptome for etiolated Arabidopsis seedlings using a structure/function approach. Funct Integr Genomics, 3: 135.

Robertson FC, Skeffington AW, Gardner MJ, Webb AA. 2009. Interactions between circadian and hormonal signalling in plants. Plant Mol Biol, 69: 419.

Romero I, Fuertes A, Benito MJ, Malpica JM, Leyva A, Paz-Ares J. 1998. More than 80R2R3-MYB regulatory genes in the genome of Arabidopsis thaliana. Plant J, 14: 273.

Sambrook J, Fritsch, E.F. and Maniatis, T. 1989. Molecular Cloning. A Laboratory Manual., Cold Spring Harbor, USA: Cold Spring Harbor Laboratory Press.

Satoh R, Fujita Y, Nakashima K, Shinozaki K, Yamaguchi-Shinozaki K. 2004. A novel subgroup of bZIP proteins functions as transcriptional activators in hypoosmolarity-responsive expression of the ProDH gene in Arabidopsis. Plant Cell Physiol, 45: 309.

Schindler U, Beckmann H, Cashmore AR. 1992. TGA1 and G-box binding factors: two distinct classes of Arabidopsis leucine zipper proteins compete for the G-box-like element TGACGTGG. Plant Cell, 4: 1309.

Shin R, Burch AY, Huppert KA, Tiwari SB, Murphy AS, Guilfoyle TJ, Schachtman DP. 2007. The Arabidopsis transcription factor MYB77 modulates auxin signal transduction. Plant Cell, 19: 2440.

Staswick PE, Serban B, Rowe M, Tiryaki I, Maldonado MT, Maldonado MC, Suza W. 2005. Characterization of an Arabidopsis enzyme family that conjugates amino acids to indole-3-acetic acid. Plant Cell, 17: 616.

Stracke R, Werber M, Weisshaar B. 2001. The R2R3-MYB gene family in Arabidopsis thaliana. Curr Opin Plant Biol, 4: 447.

Suzuki M, Kao CY, McCarty DR. 1997. The conserved B3 domain of VIVIPAROUS1 has a cooperative DNA binding activity. Plant Cell, 9: 799.

Ulmasov T, Hagen G, Guilfoyle TJ. 1997a. ARF1, a transcription factor that binds to auxin response elements. Science, 276: 1865.

Ulmasov T, Hagen G, Guilfoyle TJ. 1999a. Activation and repression of transcription by auxinresponse factors. Proc Natl Acad Sci U S A, 96: 5844.

Ulmasov T, Hagen G, Guilfoyle TJ. 1999b. Dimerization and DNA binding of auxin response factors. Plant J, 19: 309.

Ulmasov T, Liu ZB, Hagen G, Guilfoyle TJ. 1995. Composite structure of auxin response elements. Plant Cell, 7: 1611.

Ulmasov T, Murfett J, Hagen G, Guilfoyle TJ. 1997b. Aux/IAA proteins repress expression of reporter genes containing natural and highly active synthetic auxin response elements. Plant Cell, 9: 1963.

Van Loo P, Marynen P. 2009. Computational methods for the detection of cis-regulatory modules. Brief Bioinform, 10: 509.

Vanneste S, Friml J. 2009. Auxin: a trigger for change in plant development. Cell, 136: 1005.

Weiste C, Dröge-Laser W. submitted. G-box related cis-elements and their cognate bZIP transcription factors function as quantitative modulators of auxin-mediated growth responses

Wrzodek C, Schroder A, Drager A, Wanke D, Berendzen KW, Kronfeld M, Harter K, Zell A. 2010. ModuleMaster: a new tool to decipher transcriptional regulatory networks. Biosystems, 99: 79. 


\subsection{Chapter 2: G-box related cis-elements and their cognate bZIP transcription factors function as quantitative modulators of auxin-mediated growth responses*}

* This manuscript has been submitted for publication

$$
\text { Christoph Weiste }{ }^{1,2} \text { and Wolfgang Dröge-Laser }{ }^{1,2 \#}
$$

${ }^{1}$ Julius-von-Sachs-Institut, Pharmazeutische Biologie, Julius-Maximilians-Universität Würzburg, Julius-von-Sachs-Platz 2, D-97082 Würzburg, Germany

${ }^{2}$ Albrecht-von-Haller-Institut, Universität Göttingen, Untere Karspüle 2, D-37073 Göttingen, Germany

\# To whom correspondence should be addressed:

Email: wolfgang.droege-laser@uni-wuerzburg.de

Tel +49-(0)931-31-80955; Fax: +49-(0)931-31-86182

Running title: bZIP factors in auxin-mediated transcription 


\section{Abstract}

The essential plant growth hormone auxin regulates transcription via Auxin Response Elements (AuxREs) which are bound by Auxin Response Factors (ARFs). In order to sustain optimal plant growth, endogenous and environmental stimuli have to be integrated into auxin-specific transcriptional patterns. Using the Arabidopsis AtGH3.3 promoter as a model, we defined combinatorial control of auxin-mediated transcription by a complex arrangement of in part redundantly acting cis-elements consisting of AuxREs, G-BOX RELATED ELEMENTS (GREs) and MYB RESPONSE ELEMENTS (MREs). Whereas AuxREs function as auxin-dependent switches, GREs and MREs act as quantitative modulators of transcription. Importantly, GREs have been found to enhance sensitivity to low auxin concentrations. Members of the C/S1-network of basic leucine zipper (bZIP) transcription factors were identified to modulate auxinresponses via GRE elements. Gain- and loss-of-function approaches in transgenic plants demonstrate that group S1 AtbZIP2, -11 and -44 fine-tune auxin-induced transcription and alter typical auxin responses, such as root growth, lateral root formation, root hair density and gravitropism. As C/S1 bZIPs are reprogramming the primary metabolism in response to energy stress, the GRE/bZIP module is supposed to function as a "rheostat" which provides means to balance auxin-mediated growth responses depending on the energy status of the cell.

\section{Introduction}

The plant hormone auxin regulates various aspects of plant growth and development, such as embryogenesis, root and shoot architecture, organ patterning or vascular development (Abel and Theologis, 2010, Zhao, 2010). In addition, responses to environmental signals such as tropic growth or responses to pathogen attack involve auxin signalling (Müller et al., 1998, Navarro et al., 2006, Robert-Seilaniantz et al., 2007).

Auxin regulated transcription is mediated by AUXIN RESPONSE ELEMENTS (Ulmasov et al., 1995). These cis-sequences are recognised by AUXIN RESPONSE FACTORS (ARFs) (Ulmasov et al., 1997a, 1999a, d, Tiwari et al., 2003, Guilfoyle and 
Hagen, 2007), which are members of the B3-type transcription factor (TF) family (Swaminathan et al., 2008). In the absence of auxin, the ARF-mediated transcription is repressed by AUX/IAA proteins via protein-protein interaction (Gray and Estelle, 2000). AUX/IAA proteins function by interaction with the Groucho/Tup type corepressor TOPLESS (TPL) which is proposed to recruit histone deacetylases (HDACs) to the respective promoters (Liu and Karmarkar, 2008; Szemenyei et al., 2008). As histone deacetylation is correlated with transcriptional inactive, tightly packed chromatin, transcription is repressed in the absence of auxin. Auxin is recognised by binding to the F-box protein TIR1 which in turn leads to ubiquitination of Aux/IAA repressor proteins by the $\mathrm{SCF}^{\mathrm{TIR} 1}$ complex (Dharmasiri et al., 2005a, Kepinski and Leyser, 2005a). In response to auxin, Aux/IAA proteins are degraded by the $26 \mathrm{~S}$ proteasome and auxin responsive genes are transcriptionally activated by the ARFs. This regulatory mechanism based on de-repression enables rapid gene activation and has been found to be a characteristic of several hormone signalling pathways (Pauwels et al., 2010).

A general feature of transcriptional control is combinatorial regulation by several ciselements which allows the integration of diverse stimuli into transcriptional patterns. Recently, protein interaction between ARF7 and MYB77, a R2R3 MYB factor involved in regulating lateral root growth in response to environmental and nutritional cues has been demonstrated (Shin et al., 2007). MYB factors bind to MREs (MYB RESPONSIVE ELEMENTS), although no simple consensus has yet been defined. All animal MYBs and some plant MYBs bind to the MYB binding site I (MBS I; CNGTTA), whereas MBS II (AGTTAGTTA) has been defined to be recognized by many plant MYBs (Dubos et al., 2010).

Auxin induced transcription has been studied in the context of several rapidly responding groups of genes, like SAURs (SMALL AUXIN-UPREGULATED RNAs), Aux/IAAs and GH3s (Abel et al., 1995, Gil and Green, 1996). GH3 genes encode for enzymes which modify the pool of active auxin by conjugation it to amino acids (Staswick et al., 2005). The soybean GH3 gene exhibits the best-studied auxinresponsive promoter (Liu et al., 1994, Liu et al., 1997b, Ulmasov et al., 1997a). Previous findings implicate that several cis-elements are involved in auxin-mediated transcription. GH3 promoters from soybean and tobacco harbour G-BOX RELATED 
ELEMENTS (GREs; consensus: BACGTV) located in close vicinity to AuxREs. GREs are typical binding sites for basic leucine Zipper (bZIP) TFs. Although G-box binding activity has been described with respect to the soybean GH3 promoter, the corresponding TFs have not been assigned yet (Liu et al., 1997a). In our group the tobacco bZIP-factors BZI-1 and BZI-2 were found to regulate auxin-mediated $N t G H 3$ transcription in planta (Heinekamp et al., 2004, Böttner et al., 2009, Iven et al., 2010). Furthermore, conserved organisation of AuxRE and GRE promoter elements has been identified in GH3 genes from soybean and tobacco. Accordingly, a synergistic action of ARF and bZIP TFs in auxin-mediated transcription has been postulated.

Making use of the experimental advantages of the Arabidopsis system, we focussed on GREs and AuxREs with respect to their impact on combinatorial control of auxinmediated transcription. The Arabidopsis homologous of NtBZI-1 and NtBZI-2 have been classified as group C (AtbZIP9, -10, -25, -63) and group S1 (AtbZIP1, -2, -11, -44, -53) bZIPs which form specific heterodimers and therefore are proposed to be functionally interlinked (Jakoby et al., 2002, Ehlert et al., 2006). The so-called C/S1network has been implicated in reprogramming the plant's primary metabolism in response to low energy stress (Baena-Gonzalez et al., 2007, Dietrich et al., 2011) or pathogen response (Kaminaka et al., 2006). Furthermore, it is involved in source-sink control (Hanson et al., 2008) and seed storage gene expression (Alonso et al., 2009b).

Using the Arabidopsis thaliana GH3.3 as a gene model for auxin-induced transcription, we identified a complex and in part redundant network of AuxRE, MRE and GRE motifs functioning as important regulatory elements in auxin-induced transcription. Whereas AuxREs mediate auxin-specific inducibility, GREs and MREs act as quantitative modulators. In a protoplast-based screen, specific members of the C/S1 bZIP network were identified to regulate several auxin-induced promoters via the GRE motif. Using gain- and loss-of-function approaches in transgenic plants, the in planta function of these bZIPs has been demonstrated on the level of auxin-induced gene expression as well as by studying physiological and developmental auxin responses. 


\section{Results}

A 300 bp fragment of the Arabidopsis GH3.3 promoter located upstream of the ATG start is necessary and sufficient to mediate auxin responsiveness

The Arabidopsis GH3 gene family harbours 19 members (Staswick et al., 2005). In particular, subgroup II has been implicated in conjugating auxin to amino acids, resulting in biological inactive derivatives. Based on homology studies between the soybean, tobacco and Arabidopsis GH3 genes (Supplemental Figure 1A) as well as on strong similarities in promoter structure (Supplemental Figure 1B), the AtGH3.3 promoter was chosen as a gene model for further promoter analysis. In order to perform rapid analyses, the activity of $A t G H 3.3$ promoter GUS fusions (ProAtGH3.3:GUS) were tested in an Arabidopsis transient protoplast system. Physiological levels of auxin $(0.25$ $\mu \mathrm{M} \alpha$-Naphthaleneacetic Acid, NAA) were sufficient to strongly and reliably induce AtGH3.3 reporter gene activity. Using the established protoplast system, auxin responsive cis-elements were mapped by a deletion approach (Figure 1A). In order to obtain full-level auxin responsiveness comparable to the $1700 \mathrm{bp}$ full-length AtGH3.3 promoter, a nucleotide region approximately 300 bps upstream the ATG start codon was necessary and sufficient. In a complementary approach, neither the -1700 to -300 promoter fragment fused to a minimal promoter, nor a mutational derivate lacking all discernible GRE, MRE and AuxRE cis-elements transferred any auxin responsiveness to the GUS reporter. These data indicate that the $300 \mathrm{bp}$ sequence proximal to the ATG start codon (AtGH3.3-300) is required for full auxin-induced transcription.

AuxRE cis-elements mediate the auxin responsiveness of the AtGH3.3 promoter, however their contributions differ considerably

Similar to the soybean $G H 3$ promoter (Ulmasov et al., 1995) the AtGH3.3-300 promoter region harbours multiple and partially redundant cis-elements. In particular, three AuxREs were identified which display a TGTCTC (AuxRE1) and TGTCTG (AuxRE3) consensus and a related TGTCCC motif (AuxRE2) (Figure 1B). The AuxRE1 and 2 build up a module organised as tandem inverted repeats. In order to reveal the functional impact of AuxRE elements, a promoter analysis was performed in protoplasts 
making use of the mutations given in Figure 1B. As demonstrated in Figure 1C, mutations in all three AuxREs resulted in a complete loss of auxin responsiveness. Mutations in either the AuxRE1 or the AuxRE2 led to a reduction of the AtGH3.3 auxin inducibility by $20 \%$, whereas both mutations originated in an additive effect. In contrast, the mutation of AuxRE3 alone, led to an almost complete loss of auxininduced AtGH3.3 promoter activity. In summary, although all AuxREs contribute to the promoters auxin responsiveness, AuxRE3 is the most crucial cis-element. Furthermore, the AuxRE1/2 module functioned only in co-operation with the AuxRE3 and was not sufficient to establish auxin-responsive transcription by itself.

GRE and MRE cis-elements contribute to the quantitative level of auxin induced AtGH3.3 transcription

MREs and GREs have been implicated in auxin-regulated transcription (Heinekamp et al., 2004, Shin et al., 2007). Based on bioinformatic analysis, two MREs were

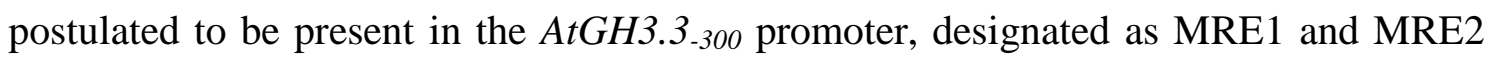
(Figure 1B). Typical plant G-boxes (CACGTG) harbour the ACGT core motif (Jakoby et al., 2002). Two G-box related sequences (BACGTV) have been identified in the AtGH3.3 promoter, named GRE2 and GRE4. Furthermore, GRE1 and GRE3 were found to overlap with TGACG-motifs. Both types of cis-elements are recognised by Gbox binding factors, whereas the latter is known to be the typical binding site for group D bZIPs (Jakoby et al., 2002).

To determine a functional role of the identified MREs and GREs in auxin-mediated gene regulation, a detailed mutational analysis of the $A t G H 3.3$ promoter was carried out in the presence and absence of applied auxin. The mutation of a single MRE (Figure 1D) or GRE (Figure 1E) elements reduced the activity of the AtGH3.3 reporter gene by 20 - 40\%. Slight quantitative differences were observed between the particular elements, e. g. MRE2 appeared to be more important than MRE1. Generally, an increasing number of mutated MREs and GREs reduced the quantitative level of auxininduced $A t G H 3.3$ expression in a non-additive way. Importantly, mutation of multiple GRE and MRE elements did not completely impair responsiveness of the AtGH3.3 promoter to auxin. A threshold induction of approximately $50 \%$ remained even if all GREs and MREs were mutated (Figure 1C). 
A

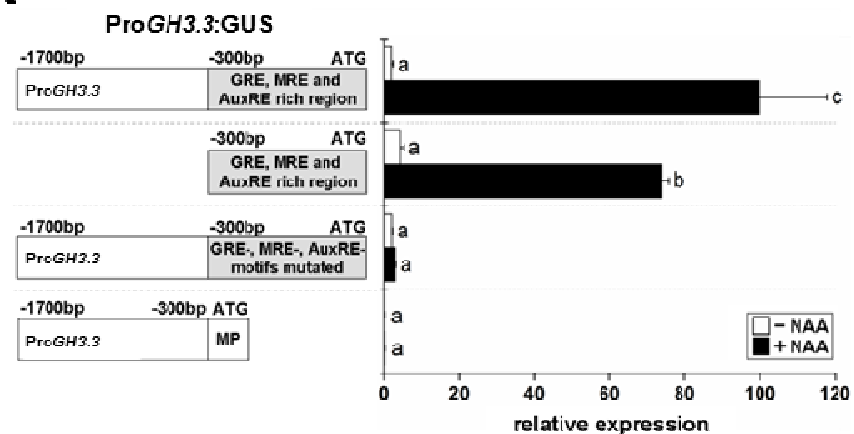

B

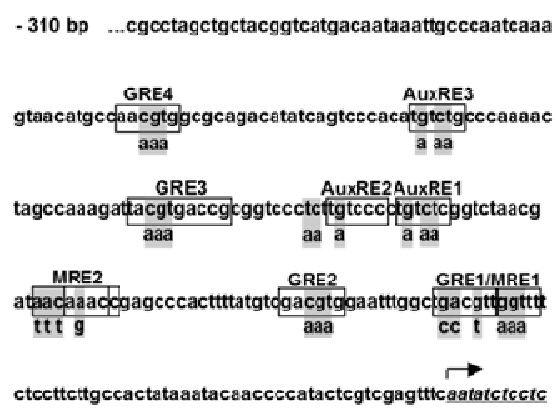

atcatcaaacacaaagtctaatattatcacttacaataccatttaalcccATG

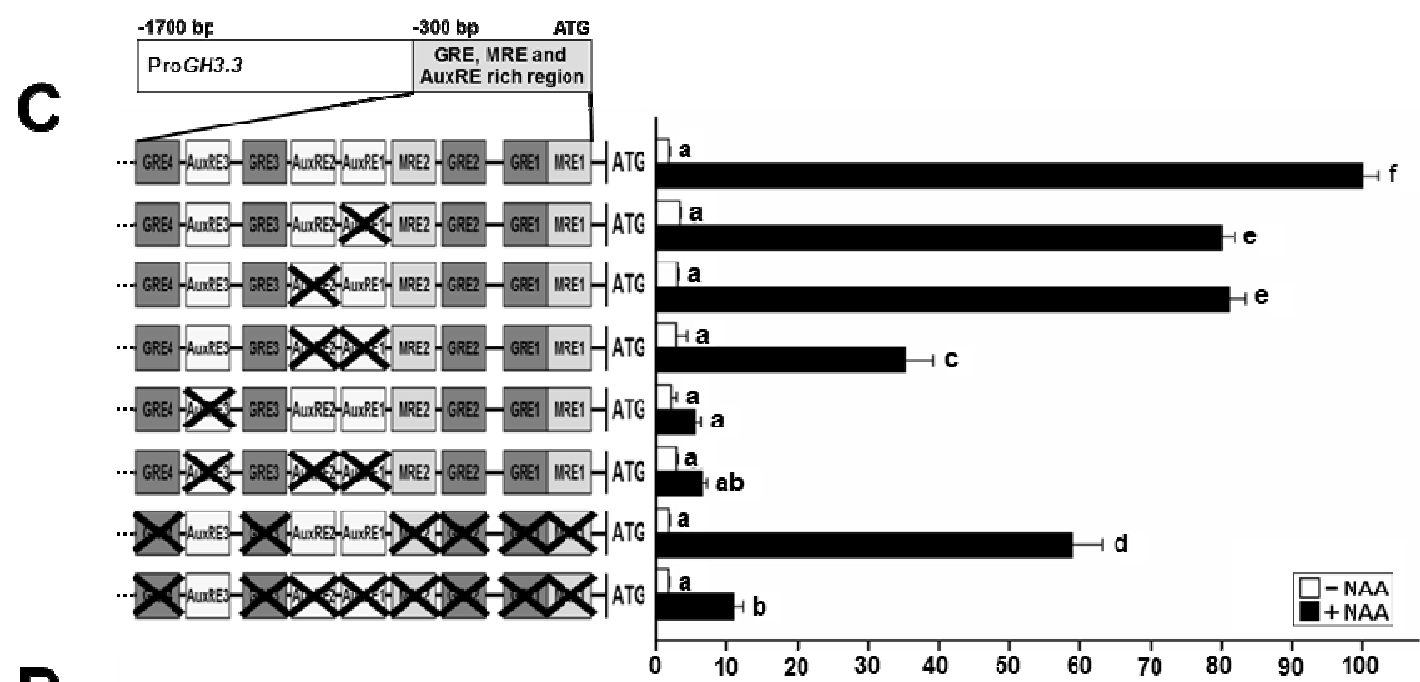

D

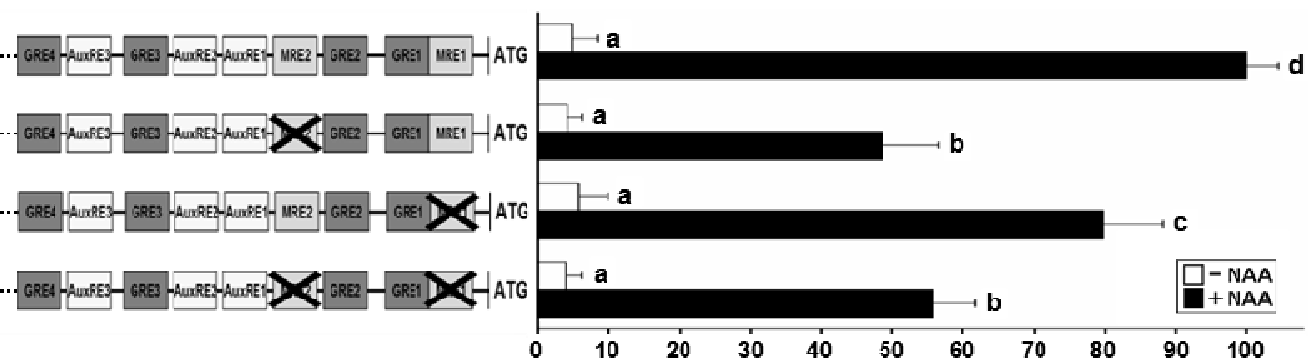

E

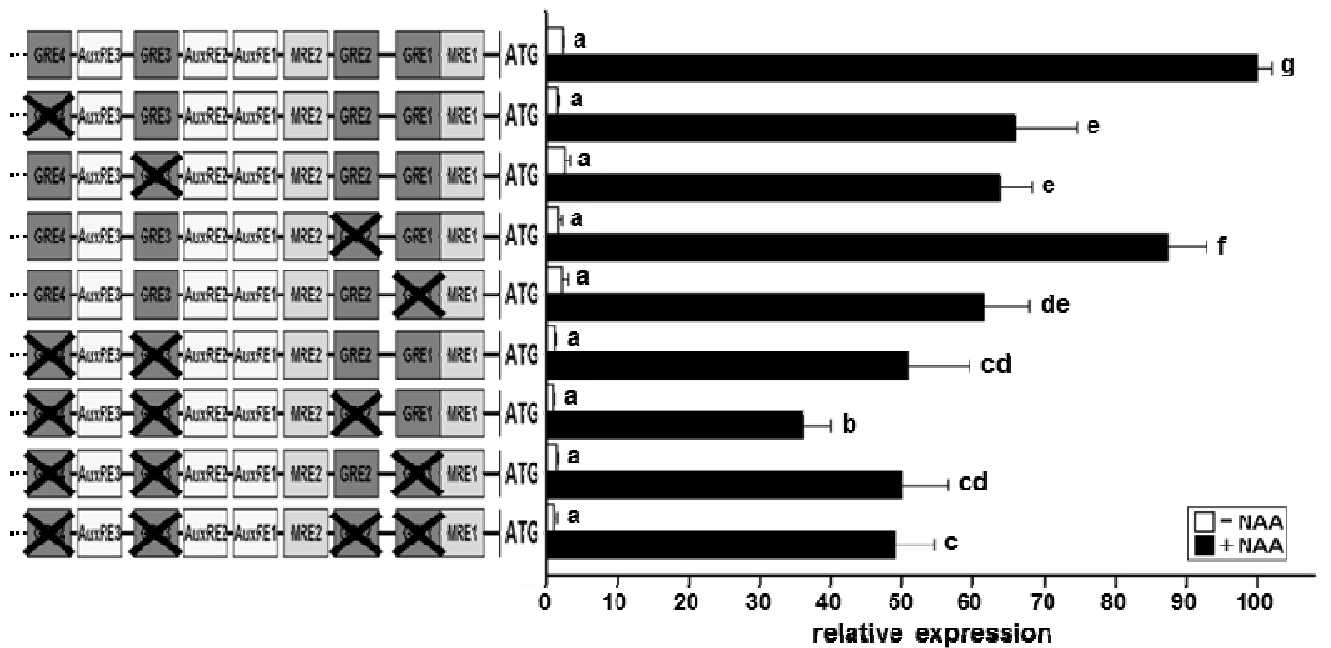


Figure 1: Analysis of the AtGH3.3 promoter in Arabidopsis protoplasts. The indicated promoter fragments have been fused to a GUS reporter gene and expression is quantified in the absence (white bars) or presence (black bars) of exogenously applied NAA $(0.25 \mu \mathrm{M}$ for $16 \mathrm{~h})$. A) Presented are results obtained with the full-length $1700 \mathrm{bp} A t G H 3.3$ promoter (ProAtGH3.3 ${ }_{-1700}$ ), the $300 \mathrm{bp}$, cis-element rich promoter region proximal to the transcriptional start site (TSS) (ProAtGH3.3 300 ), a mutational AtGH3.3. 1700 derivate which lacks all discernible GRE, MRE and AuxRE cis-elements and the remaining TSS

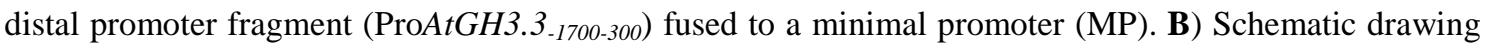
of the AtGH3.3 promoter organisation and the mutations used. Expression of the ProAtGH3.3 ${ }_{-1700}$ :GUS construct and the indicated mutational derivates has been analysed in transiently transformed mesophyll protoplasts. Given are the results obtained with mutations in AuxREs (C), MREs (D) and GREs (E). Relative positions of cis-elements and the respective mutations (X) are depicted (left). Given are mean values $( \pm \mathrm{SD})$ relative to the induction of the $A t G H 3.3_{-1700}$ promoter $(100 \%)$. Different letters denote significant differences $(\mathrm{p} \leq 0.05)$ between constructs and treatments, determined by one-way ANOVA and subsequent Fisher post-hoc test.

These data demonstrate, that although the qualitative function of the AuxREs is apparent, these elements are not sufficient to provide full auxin inducibility on their own, highlighting the impact of GREs and MREs as quantitative cis-elements.

\section{GREs are not sufficient, but functionally interact with AuxREs in auxin induced AtGH3.3 transcription}

The presence of all GREs and MREs in the AuxRE loss-of-function background did not result in any auxin-stimulated transcription of the AtGH3.3 promoter (Figure 1C). Hence, GREs and MREs are not sufficient to mediate auxin responsiveness. In order to analyse, whether there is a functional link between the most relevant AuxRE3 and a particular GRE, we reduced the complexity of the AtGH3.3 promoter by mutating the tandem AuxRE1/2 motif (Figure 2A). An additional single mutation of any GRE led to a similar reduction in auxin responsiveness, indicating that the GREs were functionally equivalent and that all GREs co-operate with AuxRE3 in auxin-mediated transcription of the $A t G H 3.3$ promoter. 
A

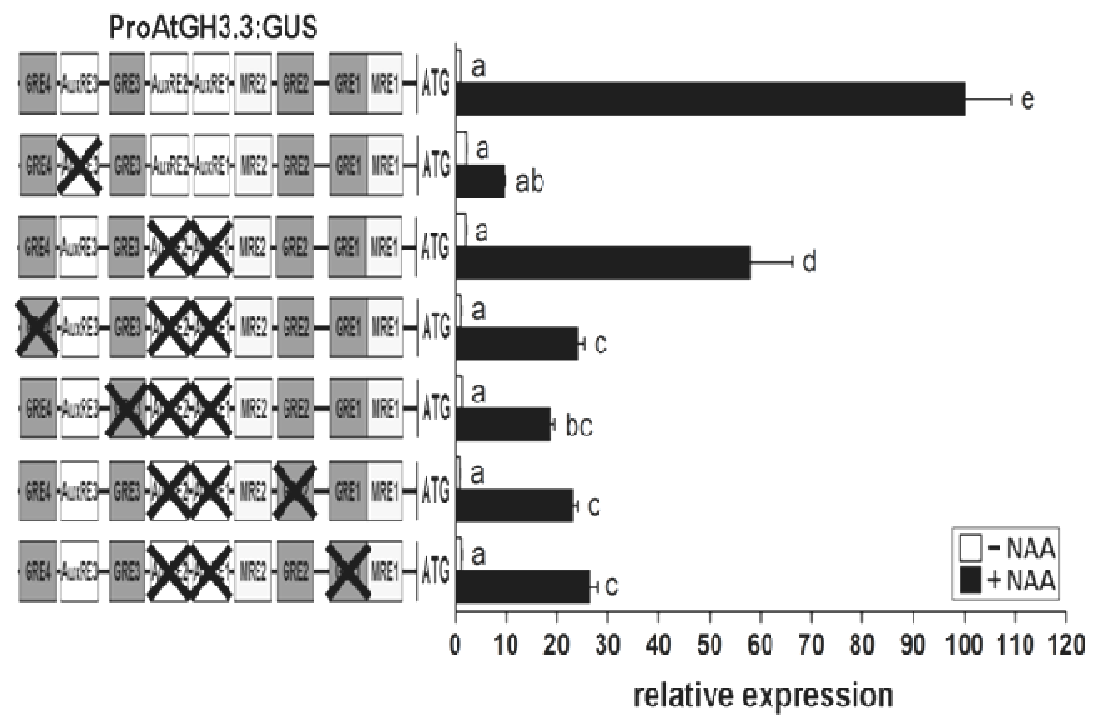

B

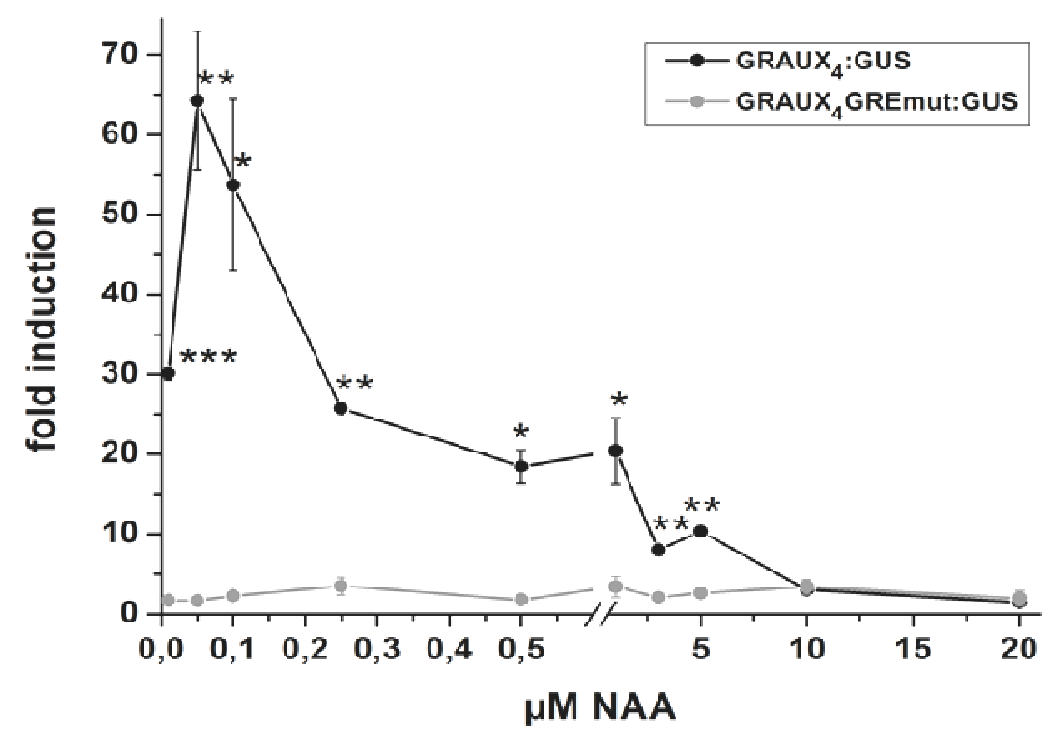

Figure 2: GREs co-operate with AuxREs and sensitize auxin induced transcription. A) AuxRE3 functionally interacts with GREs in auxin-mediated transcription. Making use of an AuxRE1/AuxRE2 mutational AtGH3.3 promoter construct, functional interaction of AuxRE3 and the respective GRE mutants can be studied. All presented GREs co-operate with AuxRE3 in a similar manner. Expression levels are presented relative to the genuine AtGH3.3. 1700 promoter $(100 \%)$. Results from mock (DMSO) and NAA treated samples $(0.25 \mu \mathrm{M}$ for $16 \mathrm{~h})$ are illustrated as white or black bars, respectively. B) GREs enhance auxin responsiveness especially to low hormone concentrations. To analyse the impact of GREs in a simplified promoter context an AtGH3.3 derived, composite GRE-AuxRE unit (GRAUX-module) has been used (Supplemental Figure 2A). Auxin responsive GRAUX tetramers have been fused to a minimal promoter in a GUS reporter construct, resulting in GRAUX $_{4}$ :GUS. Auxin triggered induction is monitored in an auxin concentration dependent manner. A mutational GRAUX construct lacking functional GREs (GRAUX ${ }_{4}$ GREmut:GUS, gray line) shows a rather weak auxin mediated expression which is auxin concentration independent, whereas the intact module (black line) is strongly auxin inducible, particularly at low concentrations. Significant differences between constructs and treatments are labelled with different letters (one-way ANOVA, followed by Fisher posthoc test; $\mathrm{p} \leq 0.05$ ) or asterisks (Students T-Test; $\mathrm{p} \leq 0.05=* ; \mathrm{p} \leq 0.01=* * ; \mathrm{p} \leq 0.001=* * *$ ). 
GREs enhance auxin responsiveness, in particular at low hormone concentrations

To study the quantitative impact of the GRE elements, an auxin concentration-response analysis was performed. Therefore, the AtGH3.3 promoter was simplified to an auxin responsive unit carrying the AuxRE1/2 inverted repeat with the adjacent GRE3, designated as GRAUX-element (GRE/AuxRE) (Supplemental Figure 2A). A multimerized GRAUX-element fused to a minimal promoter (GRAUX4:GUS) and its correspondent construct harbouring a mutated GRE was compared with respect to their auxin inducibility (Figure 2B). Whereas the GRE mutant construct showed a low and dose-independent inducibility (up to 4-fold), the presence of the functional GRE rendered the construct highly sensitive to auxin in particular to low concentrations of the hormone. Accordingly, a maximum induction of 65 fold was obtained at $0.05 \mu \mathrm{M}$ NAA. Altogether, these data suggest a rheostat function of the GREs.

\section{Particular members of the C/S1-bZIP network enhance auxin-induced AtGH3.3 transcription in protoplasts}

GREs are canonical binding sites for bZIP TFs. Based on the observation that tobacco BZI-1 and BZI-2 TFs regulate auxin responses (Heinekamp et al., 2004; Iven et al., 2010), we assayed the nine homologous Arabidopsis bZIPs (Ehlert et al., 2006). In a screening approach in protoplasts (Figure 3A), the group S1 members AtbZIP2, -11, 44, and -53 and the group C factor AtbZIP63 specifically enhanced auxin-mediated ProAtGH3.3:GUS expression, whereas other bZIPs, such as AtbZIP1 or AtbZIP9 had no impact. However, bZIP-enhanced AtGH3.3 reporter activity was not only observed when auxin was applied exogenously, but also seen in the control samples. Similar results were obtained when assaying the GRAUX GUS $_{4}$ construct (Supplemental Figure 2B). However, in contrast to ProAtGH3.3:GUS, no activation of the GRAUX $_{4}$ :GUS construct was obtained with AtbZIP63 or -53. In summary, several, presumably functionally redundant group S1 bZIP factors are involved in modulating AtGH3.3 promoter activity. 
A

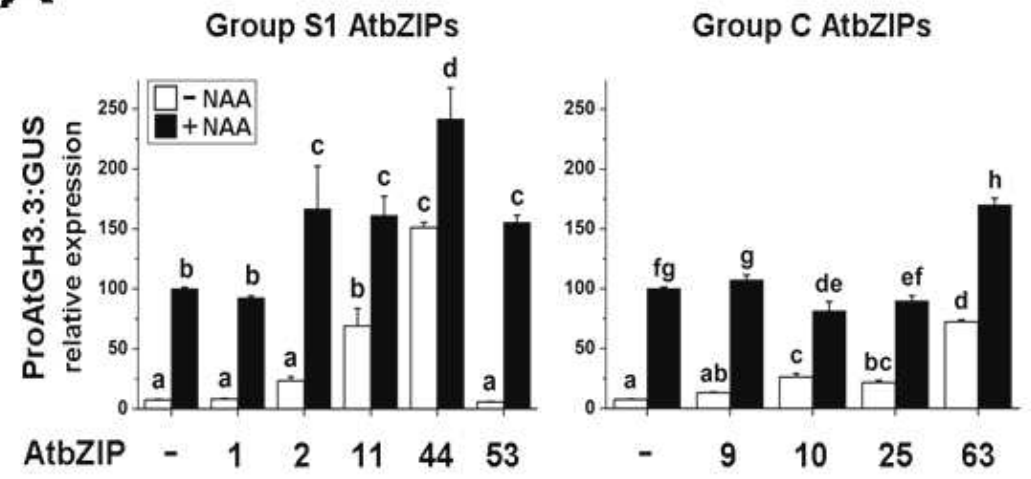

B
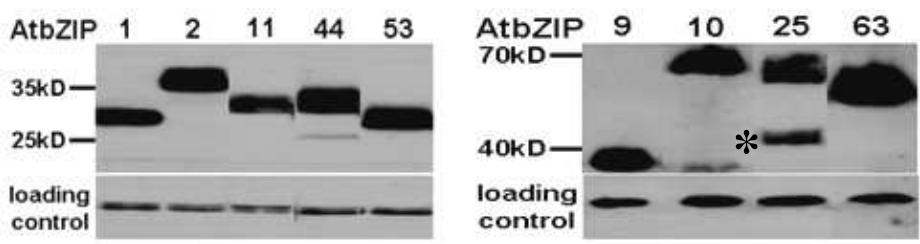

6

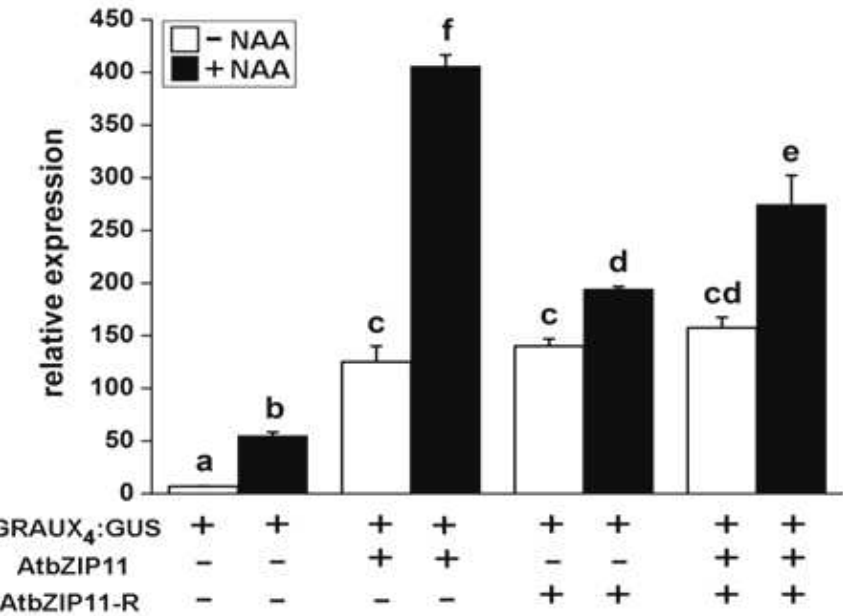

D

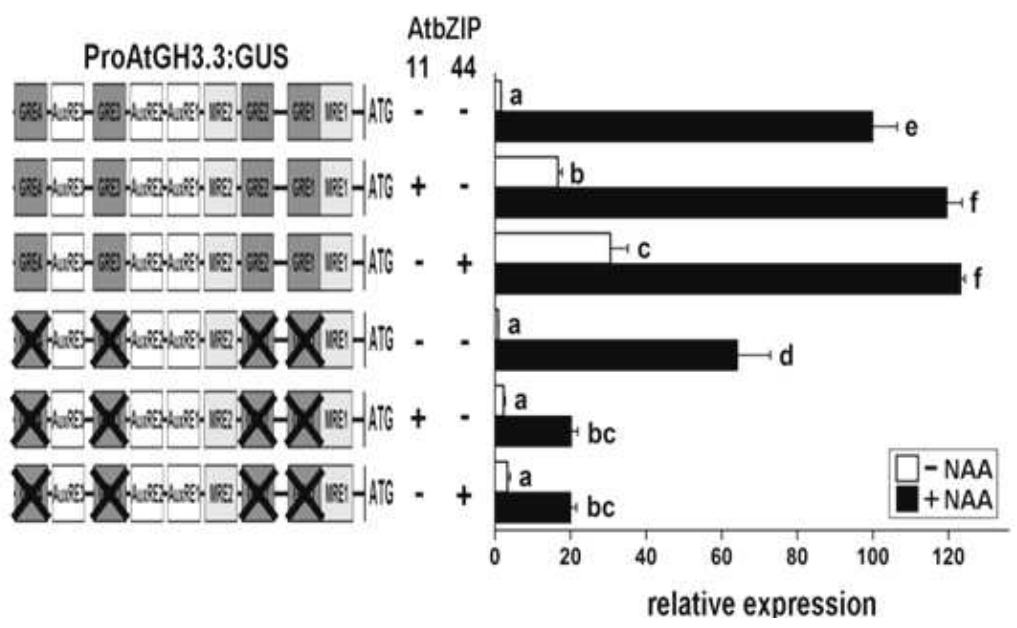


Figure 3: Particular AtbZIP TFs of the C/S1 network regulate the AtGH3.3 promoter via GRE ciselements. A) The AtGH3.3 reporter and individual Pro35S:AtbZIP-TF effector constructs have been cotransfected in protoplasts to define bZIP TF dependent alterations in AtGH3.3 driven reporter gene expression. Predominantly group S1 AtbZIPs strongly enhance reporter gene expression with and without additional auxin induction. Relative expression levels are referred to the induction of the AtGH3.3 promoter $(100 \%)$. B) Equal expression of the HA-tagged AtbZIP effectors has been confirmed by immuno-detection using an $\alpha \mathrm{HA}$-tag antibody (* degradation product). C) Expression of an AtbZIP11repressor domain fusion (AtbZIP11-R) reduces the auxin induced expression of the GRAUX4:GUS reporter. D) The contributory effect of AtbZIP11 and AtbZIP44 on AtGH3.3 expression is dependent on functional GRE cis-elements. Group S1 AtbZIP-TFs induce the AtGH3.3 driven reporter gene expression in the presence and absence of exogenous auxin application. In contrast, the effectors are incapable to activate $A t G H 3.3$ reporter derivatives in which all GRE motifs are mutated. Results from experiments using exogenous auxin $(0.25 \mu \mathrm{M}$ NAA for $16 \mathrm{~h}$ ) or mock (DMSO) treatment are visualised by black or white bars, respectively. Discrete letters designate significant differences $(\mathrm{p} \leq 0.05)$ between constructs and treatments defined by one-way ANOVA followed by Fisher posthoc test.

\section{The function of bZIPs in auxin-mediated transcription is not limited to the AtGH3.3 promoter}

In order to analyse, whether bZIP-mediated modulation of auxin-induced transcription is limited to the AtGH3.3 promoter, further GUS reporter constructs were analysed in the protoplast system using promoters of well-known auxin-induced genes which harbour GRE promoter elements (Supplemental Figure 3A). AtAux/IAA7 and -3 encode repressors of the Aux/IAA family and AtPIN4 an efflux carrier which both show a moderate and kinetically slow positive response to auxin (Abel et al., 1995, Paponov et al., 2008). In comparison to AtGH3.3, auxin-induced activation of the reporter constructs containing AtAUX/IAA3/7 and AtPIN4 promoters was rather low (Supplemental Figure 3B-D). Nevertheless, a similar set of bZIP-TFs controlled expression of all promoters analysed. This data indicate a general impact of group S1 factors AtbZIP2, -11 and -44. However, promoter specific differences were also observed e. g. in addition to these bZIPs, AtPIN4 was activated by AtbZIP10. 
Auxin-induced AtGH3.3 transcription is impaired due to expression of bZIP repressor fusions

The impact of bZIP factors was also studied by a transient loss-of-function approach using the Chimeric Repressor Silencing Technology (CRES-T) (Hiratsu et al., 2003). As a representative example, AtbZIP11 was selected and fused to a synthetic repressor domain. When bound to the respective GRE promoter motif, the AtbZIP11-R protein was supposed to block redundantly active TFs (Figure 3C). The auxin-induced activity of the GRAUX $\mathrm{G}_{4}$ :GUS construct was strongly impaired in the presence of the AtbZIP11$\mathrm{R}$ repressor fusion protein. Interestingly, the CRES-T repressor domain had no effect on the enhancing AtbZIP11 function in the absence of externally applied auxin. These data suggest that different modes of action apply for transcriptional control depending whether auxin is present or absent.

\section{AtbZIP proteins enhance auxin-induced AtGH3.3 transcription via GRE cis- elements}

In order to analyse, whether the bZIPs act via GREs, AtbZIP44 or AtbZIP11 were coexpressed with a ProAtGH3.3:GUS construct which harbours mutations in all GREs. Accordingly, AtbZIP11 or AtbZIP44 did not activate the reporter in the presence of auxin (Figure 3D, Supplemental Figure 4A, B) supporting the view that GRE binding sites are crucial for bZIP function in vivo. Surprisingly, reporter activity was strongly suppressed after bZIP co-expression. A possible explanation would be that the bZIPs induced the accumulation of AUX/IAA repressor proteins, which in turn negatively regulate the AtGH3.3 promoter via ARF interaction. This effect became more apparent when an increasing number of positively acting GRE sites within the AtGH3.3 promoter had been mutated.

\section{AtbZIP2, AtbZIP11 and AtbZIP44 control auxin-induced gene expression in planta}

In order to study the impact of bZIP factors on auxin responses at the whole plant level, transgenic gain- and loss-of function approaches were carried out. Because constitutive ectopic expression strongly interferes with plant growth (Hanson et al., 2008, Alonso et 
al., 2009b) we expressed the bZIPs as HA-tagged versions using an B-Estradiol (Est) inducible expression system (XVE) (Zuo et al., 2000). Due to a HA-tagged expression of AtbZIP11, -44 or -2, the presence of Est-induced bZIP factors could be detected by immune-blot techniques (Supplemental Figure 5A). Quantitative Real-time PCR (qRT-PCR) analysis revealed that the amount of AtGH3.3 transcripts transiently increased after Est-induction of AtbZIP11 or AtbZIP44 expression (Figure 4A), whereas the application of Est. to control plants had no effect (Supplemental Figure 5B). Consistent with the protoplast data, the in planta AtAUX/IAA3 transcript level correlated with the bZIP protein amount. The AtAUX/IAA3 and to lower extent the AtAux/IAA7 transcript levels did not show a transient expression pattern but continued to accumulate over a $24 \mathrm{~h}$ time period after Est application (Figure 4A). These data support a model in which AtGH3.3 transcription is controlled by the AUX/IAA repressors. In the moment, where Est-induced AtbZIP11 or AtbZIP44 activate the expression of Aux/IAA genes and thus the accumulation of the Aux/IAA proteins, the repression of the AtGH3.3 promoter activity started. Indeed, an accumulation of AtAux/IAA3 or AtAux/IAA7 was able to repress expression of the ProAtGH3.3:GUS reporter in protoplasts (Supplemental Figure 6A). Furthermore, in the aux/iaa7 mutant (axr2), AtGH3.3 transcription is strongly de-repressed (Supplemental Figure 6B). Altogether, these data indicate that the bZIPs modulate transcription of two counteracting systems which are connected by a negative feedback loop (see model in Figure 7).

No T-DNA insertion lines could be identified for neither AtbZIP11, nor -2 and -44, which would enable a loss-of-function approach. Furthermore, the data presented here and in previous studies strongly support a partially redundant function of these closely related bZIPs (Alonso et al., 2009b, Weltmeier et al., 2009). We therefore performed an Est-inducible artificial micro RNA (amiRNA) approach in transgenic plants (Schwab et al., 2006). The amiRNA was designed in a way that it should simultaneously target the expression of all three bZIP genes. By this approach a partial reduction of AtbZIP11, -2 and -44 transcript abundance to $25-60 \%$ of the initial level was achieved after Est. application (Figure 4B, Supplemental Figure 5C). This decrease correlated well with the partially reduced expression of AtGH3.3, AtAUX/IAA3 and AtAUX/IAA7 (Figure 4B). 
A
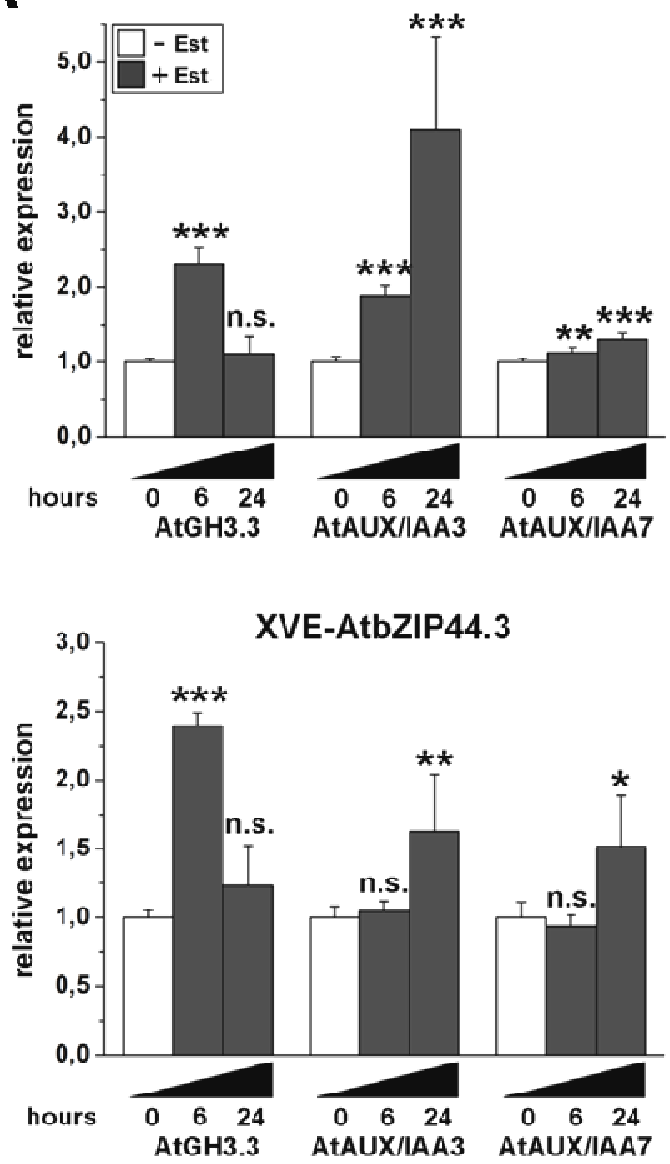

B XVE-amiRNA2/11/44.2

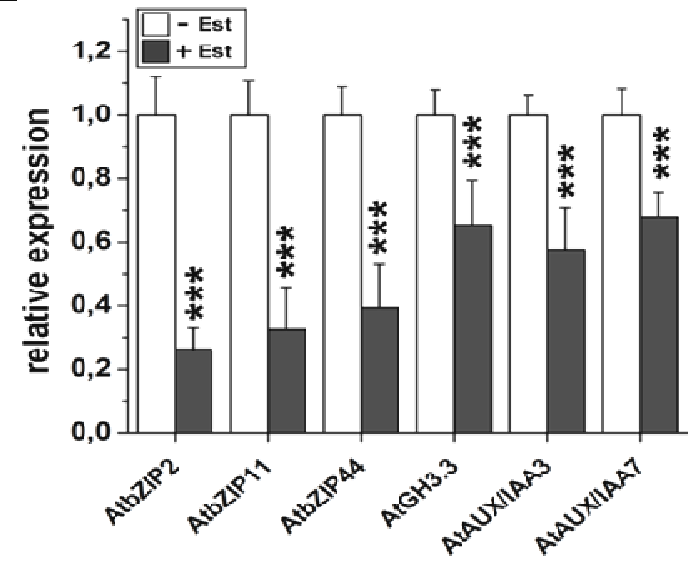

C
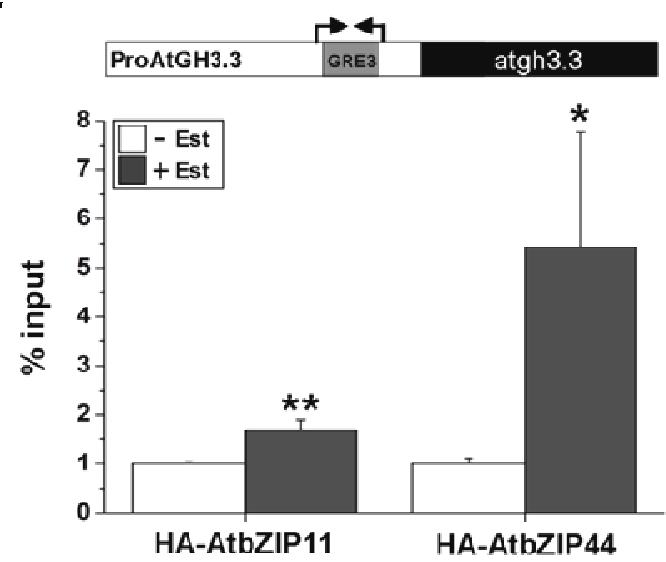

Figure 4: AtbZIP11 and -44 regulate expression of auxin-responsive genes in planta. A) Transcription of AtGH3.3, AtAUX/IAA3 and AtAUX/IAA7 has been studied by q-RT-PCR in 3 weeks-old transgenic plants which express HA-AtbZIP11 or HA-AtbZIP44 in an Est-dependent manner (Supplemental Figure 5A). Transcription of all three genes is promoted by AtbZIP11 and AtbZIP44, however their induction kinetics analysed after 0,6 or $24 \mathrm{~h}$ differ with respect to the duration of estradiol treatment analysed after 0,6 or $24 \mathrm{~h}$. Presented is the mean fold expression ( \pm SEM) compared to uninduced plants. B) Auxin induced AtGH3.3, AtAUX/IAA3 and AtAUX/IAA7 transcription is significantly diminished in an Est-inducible amiRNA knockdown line (XVE-amiRNA2/11/44 line 2). Prior to auxin induction (2 $\mu$ M NAA for $4 \mathrm{~h}$ ), AtbZIP2, -11 and -44 transcript depletion has been achieved by estradiol induced expression ( $7 \mu \mathrm{M}$ Est for $20 \mathrm{~h}$ ). C) HA-AtbZIP11 and HA-AtbZIP44 bind the AtGH3.3 promoter in vivo. BZIP binding to the GRE rich AtGH3.3 promoter region has been determined by CHIP analysis and q-RT-PCR using the primers indicated (arrow, Supplemental Table 1). Enrichment of q-RT-PCR amplified promoter fragments after Est-induced HA-AtbZIP11/44 expression is given relative to uninduced plants (defined as 1). Presented are results from 4 independent experiments including 2 individually processed pools of about 100 plants per plant line and treatment. Q-RT-PCR data have been normalised to DNA input, which is quantified by ACTIN8 transcript abundance. In all diagrams Est. induction and the corresponding mock treatment are illustrated as gray or white bars, respectively. 
In order to verify in vivo promoter $\mathrm{TF}$ interaction, Chromatin Immuno Precipitation (ChIP) experiments were performed comparing extracts from Est-inducible HAAtbZIP11 and HA-AtbZIP44 plants with and without Est-treatment. As demonstrated in Figure 4C, direct binding of the HA-tagged bZIP proteins to the AtGH3.3 promoter was detected.

\section{Expression of group S1 bZIPs modulates auxin responses}

As demonstrated in Figure 5A, the treatment of wildtype (WT) and transgenic plants with $0.25 \mu \mathrm{M}$ NAA results in auxin-related root growth phenotypes such as reduced primary root growth, enhanced lateral root formation and root hair growth. Auxin depletion should lead with respect to primary root growth to similar phenotypes (Overvoorde et al., 2010). Remarkably, some of these responses were pheno-copied in the transgenic lines which ectopically expressed AtbZIP2, -11 or -44 . For instance, bZIP expression resulted in a reduced growth of the primary root (Figure 5A, B) and an enhanced outgrowth of lateral roots (Figure 5A, C). Furthermore, Est-induced expression in XVE-AtbZIP2, -11 or -44 plants led to a significant decrease in auxinmediated root hair formation (Figure 5A, Table 1). Complementary, the downregulation of bZIP expression in Est-amiRNA plants caused a moderate, but significant reduction of lateral root formation (Figure 5C). However, no differences have been observed with respect to root length and root hair formation in these plants. Auxin had also been demonstrated to be a crucial mediator of the root gravitropism (Moulia and Fournier, 2009). Est-induced expression of all three bZIPs led to agraviotropic root growth (Figure 6A, Supplemental Figure 7). Importantly, application of auxin to these lines partially rescued the agravitropic growth phenotype. In summary it could be observed, that bZIP expression contributed in the upper root part to the typical auxinrelated responses like lateral root formation, whereas several auxin insensitive phenotypes became apparent in the more distal root tissues. 
A

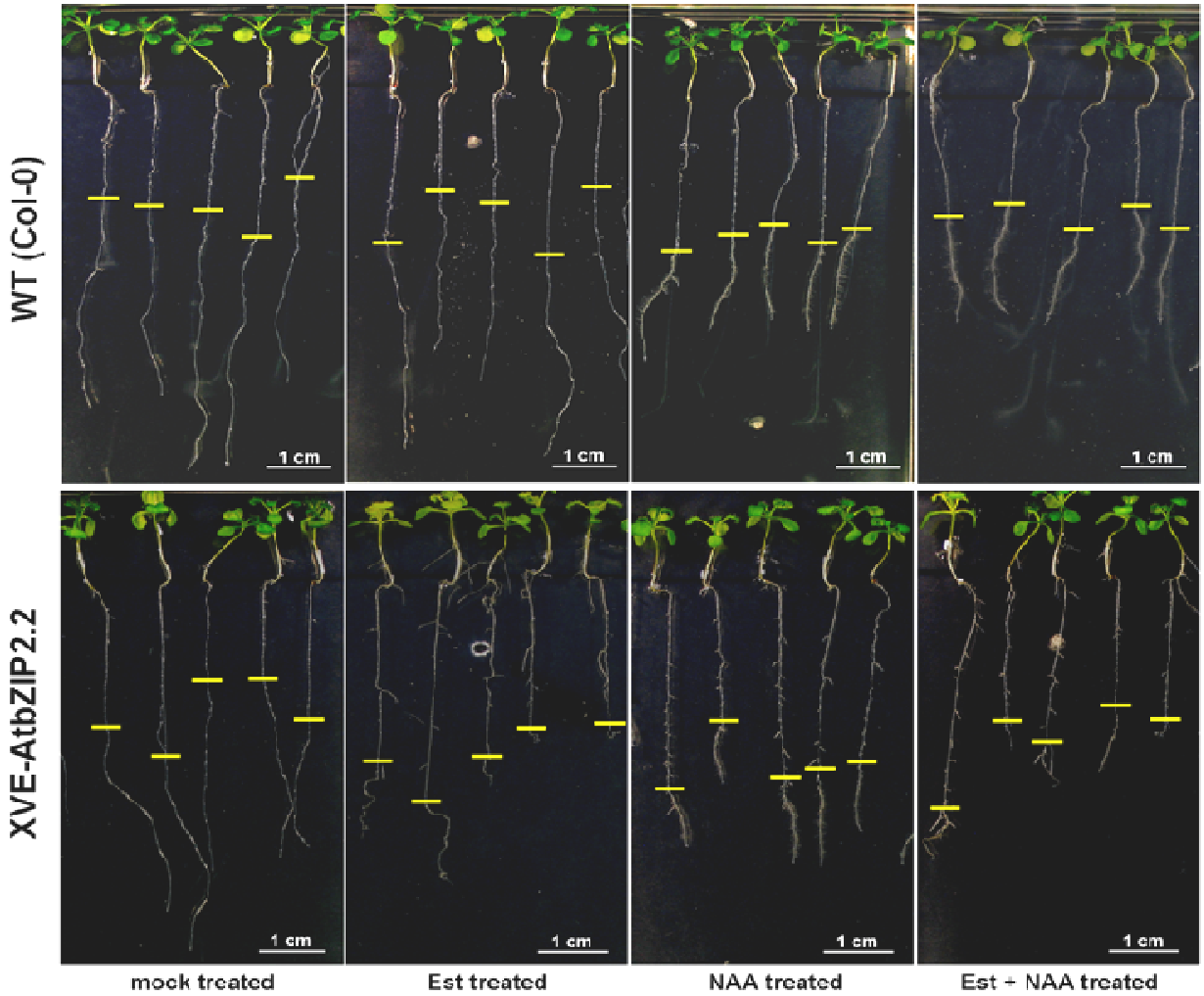

B
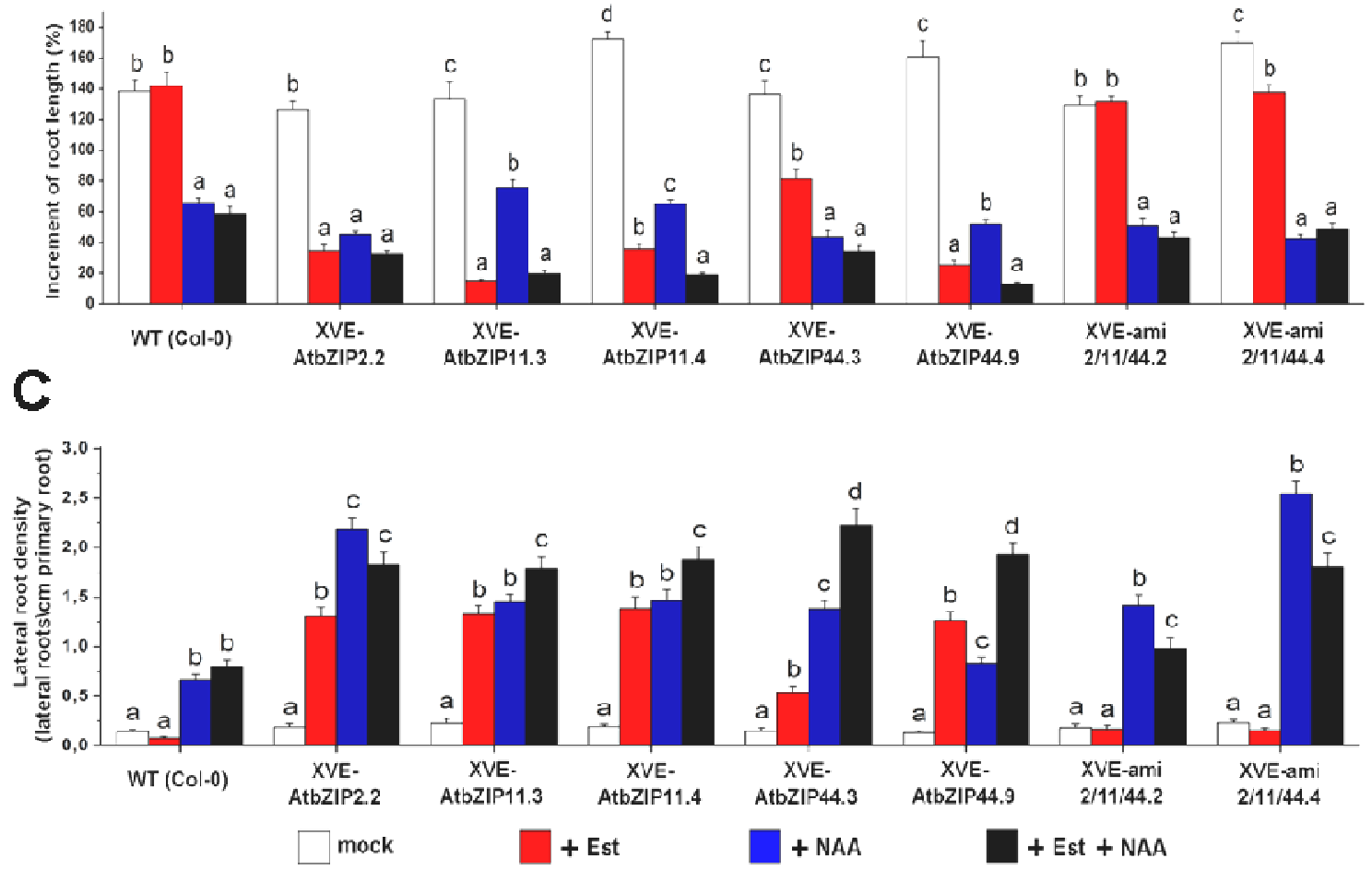

Figure 5: Plants with altered $A t b Z I P 2,-11$ or -44 expression reveal several auxin-related root growth phenotypes. A) 2 weeks-old Arabidopsis WT (upper panel) and XVE-AtbZIP2.2 (lower panel) plants have been cultivated for 7 days on MS medium supplemented with (+) or without (-) $0.25 \mu$ M NAA 
and/or $10 \mu \mathrm{M}$ Est. The most apparent auxin related root growth phenotypes of WT, XVE-AtbZIP2, -11 and -44 as well as XVE-amiRNA2/11/44 plant lines have been quantified with respect to B) the increment of root length and C) lateral root density. Mock (white), estradiol (red), NAA (blue) and combined Est/NAA (black) treatments are represented in differently coloured bars. Given are mean values $( \pm$ SEM) from 44 individual plants per treatment. Significant differences between treatments are determined by one-way ANOVA and Tukey posthoc test and denoted with different letters.

Table 1: Expression of AtbZIP2, -11 and -44 strongly attenuates auxin induced root hair formation. Auxin application $(0.25 \mu \mathrm{M}$ NAA for $7 \mathrm{~d})$ promotes local root hair formation distal to the root elongation zone. Est-induced AtbZIP2, -11 and -44 expression strongly impairs auxin-induced root hair growth. Given is the mean number of plants $( \pm$ SEM) showing no macroscopically visible root hairs in the presence of NAA or a combined NAA/Est treatment. Overall, roots from 44 individual plants per line have been analysed. Statistical significant differences have been assigned by Students T-Test and are given as p-values.

plants without root hairs (\%)

\begin{tabular}{cccc} 
Line \# & NAA treatment & NAA + Est treatment & p-value \\
\hline WT (Col-0) & $13,81 \pm 4,27$ & $18,10 \pm 4,29$ & 0,26 (n.s.) \\
XVE-AtbZIP2.2 & $11,43 \pm 2,40$ & $93,17 \pm 0,16$ & $2,22 \mathrm{E}-06$ \\
XVE-AtbZIP11.3 & $15,87 \pm 2,08$ & $93,17 \pm 0,16$ & $1,59 \mathrm{E}-06$ \\
XVE-AtbZIP11.4 & $11,43 \pm 2,40$ & $95,56 \pm 2,22$ & $6,77 \mathrm{E}-06$ \\
XVE-AtbZIP44.3 & $27,30 \pm 0,63$ & $88,73 \pm 2,06$ & $4,54 \mathrm{E}-06$ \\
XVE-AtbZIP44.9 & $13,65 \pm 3,86$ & $97,78 \pm 2,22$ & $2,32 \mathrm{E}-05$ \\
XVE-ami2/11/44.2 & $29,52 \pm 1,98$ & $31,75 \pm 1,59$ & 0,22 (n.s.) \\
XVE-ami2/11/44.4 & $22,70 \pm 2,03$ & $25,08 \pm 2,60$ & 0,25 (n.s.)
\end{tabular}

To analyse differences in auxin responsiveness at tissue and cellular level, a DR5:GFP reporter line was crossed into the Est-inducible bZIP overexpression lines (Ulmasov et al., 1997a). It has to be stressed, that this reporter does not harbour any GRE or MRE elements, but consisted of multimeric AuxRE repeats. Importantly, a reduced DR5:GFP fluorescence was recorded in the AtbZIP-overexpressing lines which correlated with agravitropic and reduced primary root growth and root hair formation (Figure 6A-C). Overall, these data suggest that the analysed bZIPs interfere with auxin response pathways in particular, in auxin distribution and/or signalling in roots. 
A

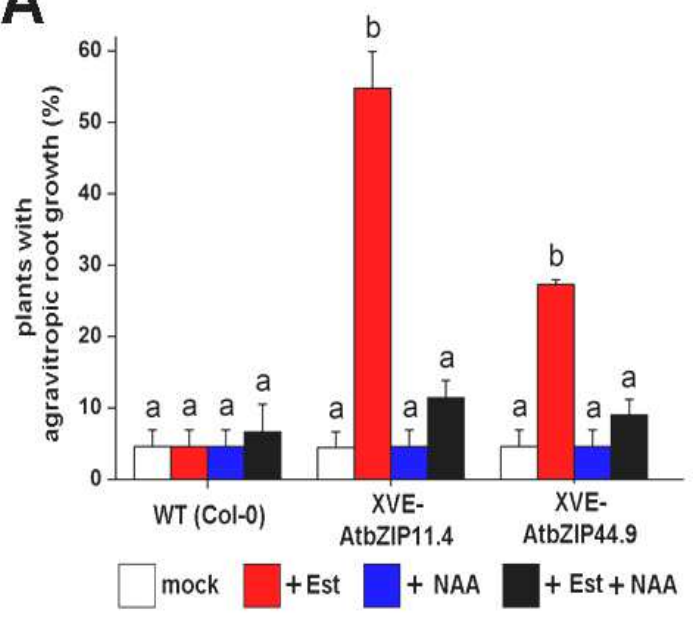

C

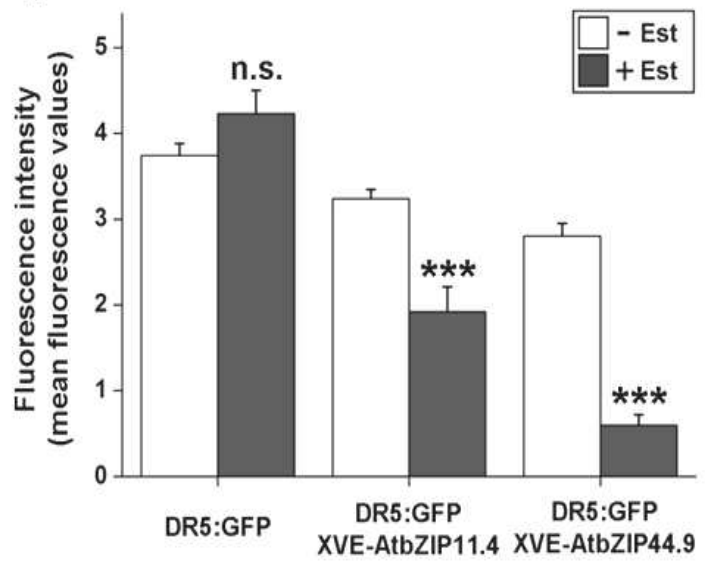

B

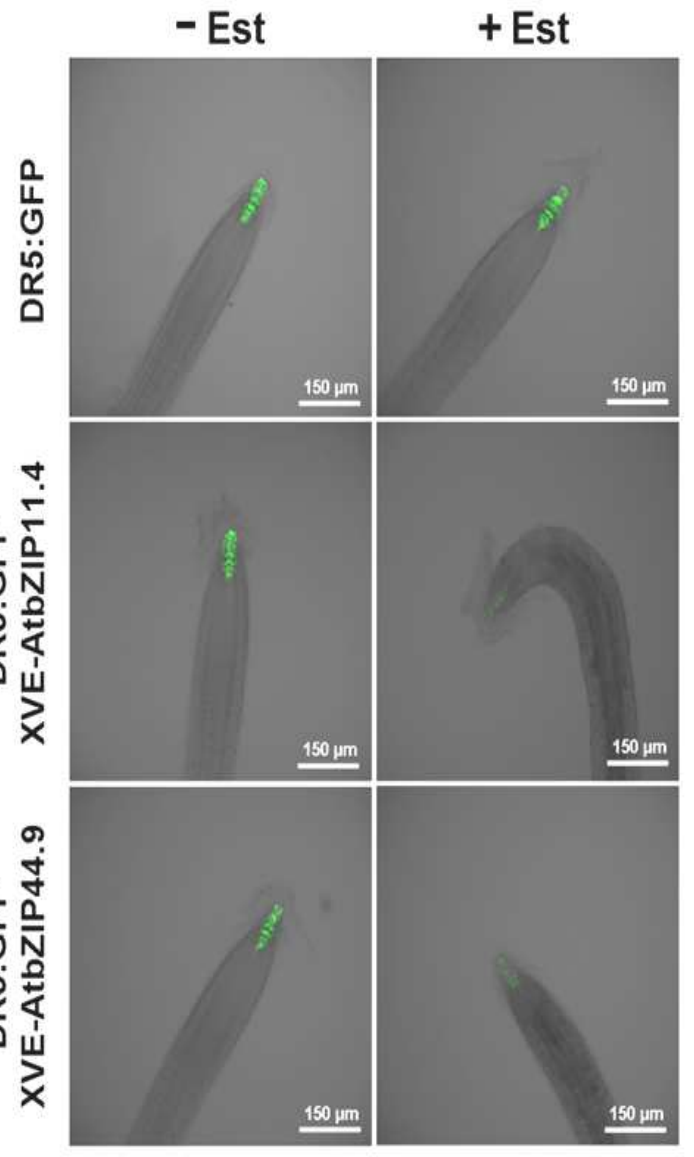

Figure 6: AtbZIP11 and -44 mediated agravitropic root growth correlates with reduced DR5:GFP activity in the root meristem. A) Number of WT, XVE-AtbZIP11 and XVE-AtbZIP44 plants (DR5:GFP background) displaying agravitropic root growth phenotypes after cultivation for 7 days on MS medium supplemented with or without Est $(10 \mu \mathrm{M})$ and/or NAA $(0.25 \mu \mathrm{M})$. Presented is the mean plant number $( \pm$ SEM) from a total of 44 individual plants tested. Treatments are illustrated by differently coloured bars, adapting the colour code from Figure 5. B) Histological analysis of auxin mediated expression in root tips of inducible AtbZIP11 and 44 over-expressors. Transgenic plants harbouring an auxin-inducible DR5:GFP reporter (upper panel) and additionally Est-inducible AtbZIP11 (XVE-AtbZIP11) (middle) or AtbZIP44 (XVE-AtbZIP44) (lower panel) constructs have been grown for $36 \mathrm{~h}$ on MS plates without (-) or with (+) addition of Est. Presented are overlays of confocal GFP fluorescence and white light images.

C) Quantification of DR5 driven GFP accumulation in the root tip. Overall, GFP fluorescence signals from root tips of 40 individual plants per treatment and plant line were analysed. White bars represent uninduced and gray bars Est. induced conditions. Significant differences between treatments are visualised by different letters (one-way ANOVA with Tukey posthoc test; $p \leq 0.05$ ) or asterisks (Students T-Test $\mathrm{p} \leq 0.05=* ; \mathrm{p} \leq 0.01=* * ; \mathrm{p} \leq 0.001=* * *)$. 


\section{Discussion}

A general characteristic of promoters is their complex composition of regulatory ciselements which integrate various inputs to establish gene specific expression patterns. Although AuxREs are well-defined auxin-dependent "on-off switches", further ciselements are required to establish quantitative expression patterns, for instance with respect of induction kinetics. Here, we investigated the biological relevance and functional interplay of these elements experimentally.

Using prevailingly an Arabidopsis protoplast system and AtGH3.3 as a model promoter, we could confirm previous findings that AuxREs are necessary and sufficient to mediate auxin responsiveness (Figure 1) (Tiwari et al., 2003). The AuxREs within the $A t G H 3.3$ promoter act in part redundantly but not in an additive manner. Depending on their position in the promoter context or its particular sequence, the impact of the AuxREs on auxin-induced transcription differs considerably. However, with respect to the AtGH3.3 promoter, it has to be pointed out that the AuxREs alone are only capable to provide less than $60 \%$ of the auxin-inducible transcriptional capacity.

Although the basic model of ARF-mediated transcription is well-established (Guilfoyle and Hagen, 2007), little is known on the functional impact of other transcriptional regulators in auxin-induced transcription. MYB77 which physically interacts with ARF7 serves as a prototypic example for co-operative regulation of auxin-induced genes, such as AtGH3.3 (Shin et al., 2007). The mutational approach presented here supports the hypothesis that MREs act as quantitative modulators of auxin-induced transcription. However, further mechanistic aspects on the function of the MRE/MYB module in gene control are still elusive. In here, we focused on a detailed analysis of GREs and its cognate TFs defining an additional "rheostat" mechanism which is finetuning AuxRE-mediated responses.

GREs co-operate with AuxREs to function as a quantitative cis-element in auxinregulated transcription

GREs are enriched within the proximal 300 bps of the AtGH3.3 promoter as well as in the set of auxin-regulated promoters from Arabidopsis. Although GREs by themselves are not sufficient to mediate auxin responsiveness (Figure 1C), they significantly 
enhance AuxRE-mediated transcription. Redundancy of this cis-element appears to be an important characteristic as multiple copies of GREs are frequently found in auxininduced promoters. Although auxin-induced transcription is stronger effected when several GREs are mutated, GREs function in a non-additive fashion. Furthermore, any of the four GREs provide a similar contribution when analysed in combination with the most effective AuxRE3 (Figure 2A). Hence, there is no requirement for correct spacing between GREs and AuxREs. This is in agreement with our observation that - in contrast to MYB77 and ARF5/7 - no direct protein interaction of the GRE binding bZIPs and ARFs could be detected using two-hybrid systems and bimolecular Fluorescence Complementation (BiFC) approaches (CW, WDL, unpublished data). However, the formation of large enhanceosome-like multi-protein complexes cannot be excluded and therefore might have been missed by the use of these techniques. Although the molecular mechanism of how GRE- and AuxRE-binding TFs corporate in transcriptional gene control remains elusive, our work establishes a second, redundantly organized, quantitative promoter input to modulate auxin-regulated transcription.

\section{BZIP transcription factors of the C/S1-network modulate auxin-dependent growth responses via GRE cis-regulatory elements}

In line with our previous findings on tobacco bZIP factors (Heinekamp et al., 2004), we could demonstrate that the closely related Arabidopsis group S1 proteins AtbZIP2, -11 and -44 enhance auxin-induced AtGH3.3 transcription (Figure 3). As the AtAuxIAA3 and -7 as well as the AtPIN4 promoters are regulated by a similar set of bZIPs, they appear to be of general importance in auxin-triggered gene expression. Although our work has focused on the three most pronounced bZIP activators of the auxin response, we cannot exclude the possibility that other bZIPs participate as well in particular, as heterodimer formation is of crucial importance within the C/S1-network (Ehlert et al., 2006; Weltmeier et al., 2006).

Redundancy among bZIP factors limits the use of loss-of-function approaches. We therefore, applied the CRES-T approach (Hiratsu et al., 2003) expressing dominant bZIP-repressor fusions. Interestingly, the AtbZIP11-R still functions as an activator when no exogenous auxin is applied, but as a repressor after auxin treatment. As 
demonstrated by Szemenyei et al. (2008), transcription of auxin-controlled genes is blocked in the absence of auxin by AUX/IAA/TPL-dependent recruitment of HDACs. The AtbZIP11-R repressor recruits the same histone deacetylation machinery however, it functions independent of an auxin-mediated protein degradation mechanism. Accordingly, as AtbZIP11-R is proposed to specifically target GREs in the AtGH3.3 promoter, it will reduce its transcription even in the presence of auxin. Interestingly, both AtbZIP11 and AtbZIP11-R functions as activators when no auxin is added. Under these conditions, transcription is repressed by the AUX/IAA/TPL complex which cannot be further enhanced by an additional repressor domain. Nevertheless, the activator function of AtbZIP11 becomes obvious, which is able to bypass the AUX/IAA repression mechanism when overexpressed. We therefore conclude that the activator function of AtbZIP11 is discrete from the AUX/IAA repressor system.

In vivo binding of bZIPs to the AtGH3.3 promoter has been demonstrated by ChIP. To confirm whether binding is mediated by GREs, co-expression analyses in protoplasts were performed using the ProAtGH3.3:GUS construct. Mutation of all GREs impairs the impact of bZIPs to activate the promoter, which supports our hypothesis that GREs act as in vivo binding sites.

The data obtained in the protoplast system was further substantiated by transgenic approaches. A balanced expression of AtbZIP2, -11 and -44 appears to be very critical as no viable overexpression or knock-out lines could be obtained (Hanson et al., 2008, Weltmeier et al., 2009). In particular, all constitutive overexpressors are dramatically dwarfed. In contrast, the related group S1 AtbZIP1 does not enhance auxin-induced transcription and accordingly, the respective transgenic approaches could successfully be established (Dietrich et al., 2011). These data suggest that particular C/S1-network members display specific functions. Making use of Est-induced bZIP expression, typical auxin-related root phenotypes were observed (Figure 5). As the expression of either AtbZIP2, -11 or -44 leads to similar responses, these data imply an at least partially redundant function. In the upper root part lateral root formation was enhanced, whereas several auxin insensitive phenotypes, which are reminiscent to those of auxininsensitive mutants such as the gain-of function shy2-2 (aux/iaa3) mutant (Weijers et al., 2005) were apparent in the more distal root tissues. As endogenous auxin is present in the transgenic plants, we interpret these findings as enhancer function of the bZIPs on 
auxin-modulated processes which have already been established.

Ectopic overexpression of TFs may generate unspecific (neomorphic) phenotypes. As knock-out lines appear to be lethal, Est-inducible multi-target amiRNA lines have been established as an alternative loss-of-function approach which simultaneously downregulate transcript abundance of AtbZIP2, -44, -11. As bZIP expression is only partially reduced, these lines are viable but show only moderate phenotypical alterations. However, these lines displayed significantly reduced auxin-induced lateral root formation, which is complementary to the results obtained by overexpression.

The physiological and developmental findings are corroborated by a reduced expression of a DR5:GFP reporter gene in the root tip of the Est-inducible bZIP overexpressors. Overall, enhanced lateral root formation in the upper part of the root compared to auxin insensitive phenotypes in the most distal root areas (reduced primary root growth, agravitropic root growth, and reduced root hair formation) point to an altered auxin distribution. Indeed, recent studies reveal an important role of the bZIP target genes AtAUX/IAA3 (shy2) and AtAUX/IAA7 (axr2) which encode repressor proteins controlling root growth by altering auxin distribution. With respect to agravitropic root responses, primary root and root hair growth, constitutive expression or expression of gain-of function variants of these AUX/IAA proteins (Liscum and Reed, 2002) partially pheno-copies the bZIP-induced root growth alterations. Moreover, it has been reported that especially SHY2 is involved in determining the root meristem size by repressing PIN1, PIN3 and PIN7 expression, which are crucial facilitators of polar auxin transport to the root tip (Blilou et al., 2005, Moubayidin et al., 2010, Scarpella et al., 2010). This leads to a local reduction of auxin and thereby to a change of the auxin/cytokinin balance which controls meristem growth. When auxin concentrations decrease which contribute to meristem growth, the cytokinin induced cell differentiation in the meristem exceeds and root growth is reduced. To confirm this hypothesis, measurements of local auxin concentrations are needed but difficult to perform.

\section{BZIP transcription factors of the C/S1 network sensitise auxin signalling by interfering with the $A U X / I A A$ - $G H 3$ feed-back mechanism}

Auxin rapidly acts on activation of transcription but even more important, an efficient 
mechanism is needed to reverse the system back to its initial state. Inactivation of a "brake" mechanism has frequently been described for several plant and animal signalling systems. Rapid regulation of genes including AtGH3.3 by auxin is mediated due to the degradation of the AUX/IAA repressors ("release of the brake"). Accumulation of the AtGH3.3 enzyme initiates a feed-back mechanism which inactivates auxin by amino acid conjugation (Staswick et al., 2005). The reduced pool of free and active auxin leads to a decelerated AUX/IAA degradation and, as a consequence, to a reinforced repression of the early auxin responsive genes including AtGH3.3. As pointed out in Figure 7, GREs and the GRE-binding bZIPs participate in modulation of this feed-back mechanism by controlling transcription of AtGH3.3 and AtAux/IAA. As demonstrated in the dose-response analysis, GREs sensitise the cell to respond particular strong to low auxin levels (Figure 2B). Hence, signal input via GREs acts on a very sensitive regulatory circuit in auxin response. It can be anticipated that due to differences in the promoter context, specific induction kinetics and expression levels of repressor and target genes are established, which build-up the self-regulating feedback loop. Altogether, this system provides a sophisticated means to fine-tune kinetic properties of the auxin signalling network and in consequence functions as a "rheostat" to regulate auxin responses. Computational modelling of the experimentally obtained data is needed to clearly define the quantitative impact of the GRE/bZIP system on the auxin response.

\section{Due to its function in low energy signalling, the C/S1 network is proposed to integrate information about the energy status into auxin specific transcriptional patterns}

Recently, we and others could demonstrate that members of the C/S1-network of bZIP factors play an essential role in SnRK1 (SNF1 RELATED KINASE1) mediated low energy signalling, by adjusting the plant metabolism to energy starvation conditions (Baena-Gonzalez et al., 2007, Dietrich et al., 2011). In here, we present evidence for an important signalling gateway of the C/S1-network to modulate auxin-mediated transcription during nutrient deficient conditions and thus, provide a first insight into a central growth-regulatory mechanism in plants. 
A number of studies have established a functional link between auxin-mediated growth responses and stress defense, e. g. auxin-mediated growth regulation is "hijacked" by plant pathogens (Robert-Seilaniantz et al., 2007). An understanding how the plant is integrating environmental stress signals and information on the cellular energy status into growth-specific transcriptional patterns will be crucial both for basic science and future agronomical approaches to improve plant performance.

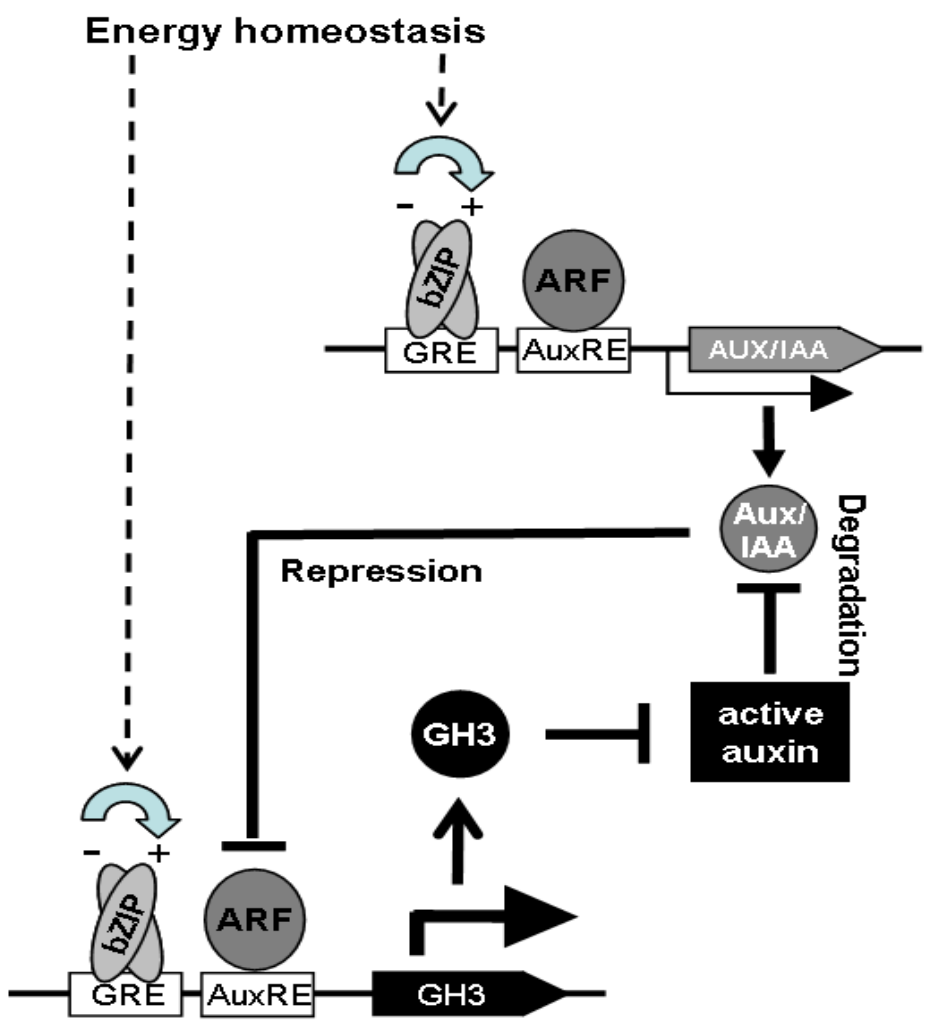

Figure 7: Model describing the function of group S1 bZIP factors in auxin-regulated transcription. Auxin induced transcription of AtGH3.3 is established by ARF/AuxRE interaction and further enhanced by AtbZIP2, -11 and -44 which bind GREs as quantitative cis-elements. The AtGH3.3 encoded enzymatic activity is proposed to reduce the pool of active auxin by conjugating it to amino acids. Decreasing amounts of active auxin would lead to reduced degradation of AUX/IAA repressor proteins and consequently to enhanced repression of ARF regulated AtGH3.3 transcription. This self-regulating feedback loop is modulated by the GRE/AtbZIP "rheostat" system. 


\section{Material \& Methods}

\section{Plant material and culture}

For cultivation and transformation of Arabidopsis thaliana ecotype Columbia (Col-0) and the axr2 (aux/iaa7)(Wilson et al., 1990) mutant, plants were grown on soil under long day conditions ( $16 \mathrm{~h}$ light $/ 8 \mathrm{~h}$ dark) at $23{ }^{\circ} \mathrm{C}$ and a relative humidity of $60 \%$. Transgenic lines were generated using the "Floral Dip Transformation" technique applying the Agrobacterium tumefaciens strain GV3101 (Weigel and Glazebrook, 2002).

For expression analyses and root morphology assays surface sterilized and stratificated seeds were cultivated on 1/4 MS (Murashige and Skoog, 1962) agar plates without sugars under long day conditions. Prior to expression analysis by q-RT-PCR three weeks old Est inducible plants were treated with $7 \mu \mathrm{M}$ Est (17-ß-estradiol-E2758, Sigma-Aldrich Chemie GmbH, Munich, Germany) in PBS or an appropriate mock solution for 6 or 24 $\mathrm{h}$, respectively. For expression analysis of amiRNA lines, the plants were preinduced with $10 \mu \mathrm{M}$ Est for $20 \mathrm{~h}$ to ensure target mRNA degradation prior to gene induction by an additional auxin treatment $(2 \mu \mathrm{M}$ NAA for $4 \mathrm{~h})$.

Plants analysed in root morphology assays were grown for two weeks on agar plates in vertical position before they were transferred on inductive medium $(0,25 \mu \mathrm{M}$ NAA and $10 \mu \mathrm{M}$ Est) or the respective mock treatment. Root morphology or DR5:GFP expression was monitored after one week or $36 \mathrm{~h}$, respectively.

\section{Protoplast transformation}

Protoplast transformation has been performed as described by (Sheen, 2001) with modifications according to Ehlert et al. (2006). For auxin treatment, protoplasts were incubated over night in WI solution supplemented with $0.25 \mu \mathrm{M}$ NAA. In standard experiments $9 \mu \mathrm{g}$ of reporter plasmid, $3 \mu \mathrm{g}$ of a NAN normalization plasmid (Kirby and Kavanagh, 2002) and $14 \mu \mathrm{g}$ of an effector plasmid has been used. If not stated otherwise, mean values are calculated from 6 independent transfections. 


\section{Molecular biological techniques}

Standard DNA techniques have been described in (Sambrook et al., 1989). DNA sequence analyses were performed using an ABI310 sequencer (Applied Biosystems, Darmstadt, Germany) with an ABI PRISM BigDye terminator cycle sequencing reaction kit (Applied Biosystems, Darmstadt, Germany). Vector DNA was gel extracted and prepared using commercial kits (Macherey-Nagel GmbH \& Co. KG, Düren, Germany). Western analysis has been performed making use of a polyclonal $\alpha$-HA antibody from rabbit (Santa Cruz, Santa Cruz, CA, USA) and an anti-rabbit IgG conjugated with a horseradish peroxidase (GE Healthcare, Freiburg, Germany). Q-RTPCR has been performed as described in Dietrich et al. (2011) and ChIP as described in Weltmeier et al. (2006) with modifications according to Fode et al., (2009). Oligonucleotide primers are summarized in Table S1.

\section{Vector construction}

The promoter:GUS reporter constructs used in the transient protoplast transactivation assays were created by amplifying the promoter sequences including the 5 UTR from AtGH3.3 ( $1700 \mathrm{bp})$, AtAUX/IAA3 ( $2060 \mathrm{bp})$, AtAUX/IAA7 ( $2060 \mathrm{bp})$ and AtPIN4 ( $3000 \mathrm{bp}$ ) using Arabidopsis WT genomic DNA and the PCR primers listed in Table S2. Making use of the attached flanking restriction sites of the resulting PCR products (AtGH3.3: XbaI, NcoI; AtAUX/IAA3 and AtAUX/IAA7: BcuI, NcoI; AtPIN4: PstI, NcoI) the promoter fragments were inserted in the reporter plasmid pBT10-GUS (B. Weisshaar, University of Bielefeld, Germany). The ProAtGH3.3 ${ }_{-1700-300}$ derivative was generated accordingly using XbaI / NcoI restriction sites. The ProAtGH3.3 $3_{-300}$ :GUS construct were created by removing the upstream promoter region $(300-1700 \mathrm{bp})$ from the pBT10-AtGH3.3:GUS construct by digestion with BsiWI and XbaI, subsequent fillin of the resulting sticky-ends with Klenow Fragment (Fermentas, Germany) and bluntend ligation of the vector. For site directed mutagenesis of the AuxRE, GRE and MRE cis-elements within the AtGH3.3 promoter, the Quick Change site directed mutagenesis kit (Stratagene, Amsterdam, Netherlands) was used following the manufacturers manual. The primers were designed according to: http://www.stratagene.com/ qcprimerdesign and are listed in Table S1. 
The synthetic GRAUX-unit was designed using complementary oligonucleotides for the corresponding sequence with a 5 flanking EcoRI and BcuI and a 3 flanking XbaI restriction site. After hybridisation of the oligonucleotides the fragment was ligated in the plasmid pBT10-GUS and multimerized according to (Rushton et al., 2002).

The AtbZIP group S1 and C bZIP effector plasmids used in the transactivation assays have been described in Ehlert et al., (2006), entry clones for AtAUX/IAA3 and AtAUX/IAA7 were obtained from the REGIA TF collection (Paz-Ares et al., 2002) and were transferred into the pHBTL expression vector (Ehlert et al., 2006). The transient expression vector pEHA-EAR used to generate TF-repressor domain fusions is described in Dietrich et al. (2011).

In order to generate stable transformed Arabidopsis plants the Est. inducible XVE system was used. The gateway compatible binary pMDC7 vector (Zuo et al., 2000) was applied for inducible expression of amiRNA constructs. Target specific and efficient amiRNA sequences were identified using the online amiRNA design tool WMD2 at http://wmd2.weigelworld.org.

To generate Est-inducible overexpressors and to monitor target gene expression a HAtag was incorporated into the pMCD7 vector. The HA-tag was PCR amplified from the pEHA vector (Weiste et al., 2007) attaching XhoI restriction sites and inserted between the inducible promoter and the gateway attachment site of the pMDC7 vector.

\section{Quantification of auxin responses in Arabidopsis roots}

To determine root morphology parameters high resolution images (5 Megapixel, 24 bit) of 44 individual plants per treatment were taken using the Camag reprostar 3 documentation system with a Canon G5 camera (CAMAG AG \& Co. GmbH, Berlin, Germany). From these pictures the root parameters of the differently treated plants were monitored. These are: lateral root number, presence or absence of macroscopically visible root hairs, the abundance of roots with obvious agravitropic root growth (at least one root reorientation of more than $\sim 45^{\circ}$ ) and the total root length before and after one week of treatment. The root length was measured using the Image $\mathrm{J} 1.43 \mathrm{u}$ software available at http://rsb.info.nih.gov/ij whereas the number of lateral roots, roots 
withlwithout root hairs and roots with agravitropic growth was determined by manual counting.

\section{Confocal microscopy}

To determine the GFP expression driven by the auxin inducible DR5:GFP reporter within the root, 200 fold enlarged brightfield and fluorescence images of 40 individual root tips per treatment were taken using the Leica SP5 confocal microscope. Fluorescence intensities were quantified as relative fluorescence intensity units using the Leica AF lite application suite 2.0.0.

\section{Phylogenetic and cis-element analysis}

Phylogenetic analysis of group II GH3 proteins from Arabidopsis thaliana, Glycine max and Nicotiana tabacuum was performed online at http://www.phylogeny.fr using the MUSCLE, PhyML and TreeDyn algorithms for sequence alignment, phylogeny analysis and tree rendering. Protein sequences from Arabidopsis GH3s were obtained from TAIR and for NtGH3 (AF123503) and GmGH3 (CAA42636) from NCBI. The ciselement analysis and motif visualisation within the analysed promoter sequences was performed using the Toucan 2 software at: http://homes.esat.kuleuven.be/ saerts/ software/toucan.php. Promoter sequences for Arabidopsis GH3 genes were obtained from TAIR and for $N t$ and $G m G H 3$ genes from NCBI.

\section{Statistics}

Figures and statistical tests were done applying the OriginPro 8.1G and Statgraphics Centurion XVI software. Significant differences between multiple constructs and treatments were determined using the One-way ANOVA test followed by a Fisher posthoc test $(\mathrm{p} \leq 0.05)$ and are visualized by different letters. Significant differences between only two datasets are defined making use of the Students T-Test and are labeled with asterisks $(\mathrm{p} \leq 0.05=* ; \mathrm{p} \leq 0.01=* * ; \mathrm{p} \leq 0.001=* * *)$. 


\section{Acknowledgements}

We are grateful to Anna Hermann and Jennifer Krüger for valuable technical assistance and K. Harter for proof reading. We thank B. Weisshaar (University of Bielefeld, Germany) and N.-H. Chua (Rockefeller University, NY, USA) for plasmids and C. Gatz, V. Lipka and J. Schirawski (University of Göttingen, Germany) for sharing lab facilities. This research was supported by grants from the Deutsche Forschungsgemeinschaft (DFG DR273-10/2).

\section{Accession numbers}

Arabidopsis Genome Initiative identifiers for the genes mentioned in this article are as follows: AtbZIP2 (At2g18160), AtbZIP11 (At4g34590), AtbZIP44 (At1g75390), AtbZIP53 (At3g62420), AtbZIP1 (At5g49450), AtbZIP63 (At5g28770), AtbZIP10 (At4g02640), AtbZIP25 (At3g54620), AtbZIP9 (At5g24800), UBI5 (At3g62250), AtGH3.3 (At2g23170), AtAUX/IAA3 (At1g04240), AtAUX/IAA7 (At3g23050), AtPIN4 (At2g01420).

\section{Supplemental Data}

Supplemental Figure 1. Analysis of group II $G H 3$ promoters from diverse plant species.

Supplemental Figure 2. Group S1 AtbZIP2, -11 and -44 strongly promote transcription of the AtGH3.3 derived GRAUX-module.

Supplemental Figure 3. Particular group C/S1 AtbZIPs regulate auxin-responsive promoters.

Supplemental Figure 4. Group S1 AtbZIPs regulate the $A t G H 3.3$ promoter via GRE cis-elements.

Supplemental Figure 5. The XVE system enables controlled AtbZIP expression by low estradiol concentrations, which do not unspecifically alter gene expression in WT plants.

Supplemental Figure 6. AtGH3.3 expression is negatively regulated by AtAUX/IAA3 and AtAUX/IAA7. 
Supplemental Figure 7. Expression of AtbZIP2, -11 or -44 leads to agravitropic root growth.

Supplemental Table 1. List of oligonucleotide sequences used in this study.

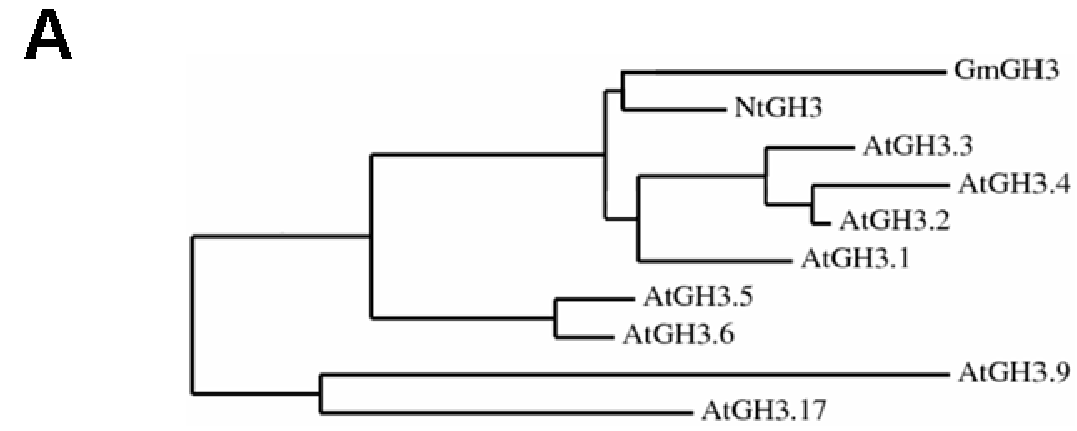

B
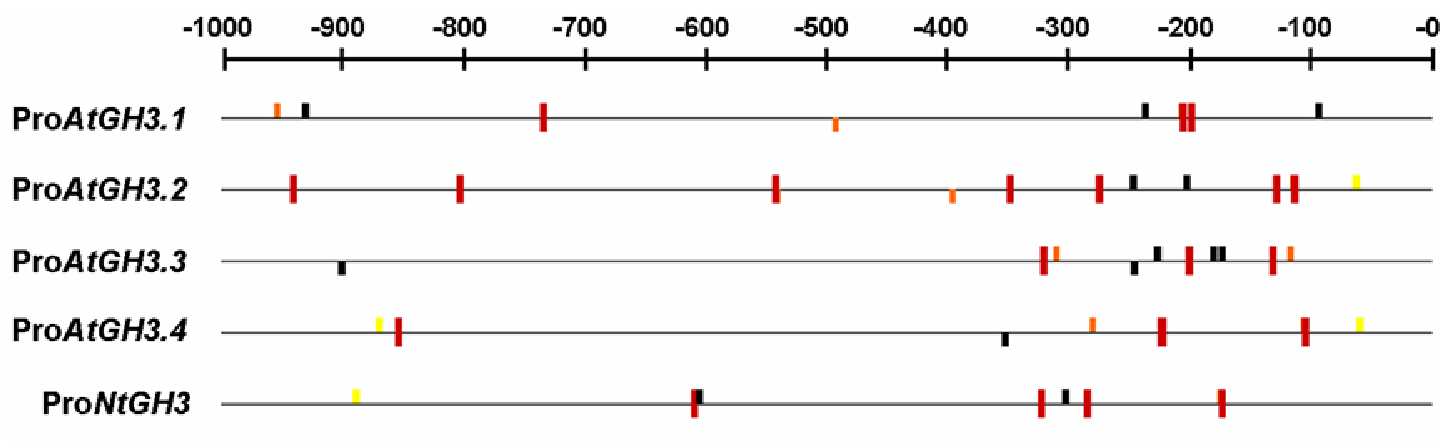

ProGmGH3

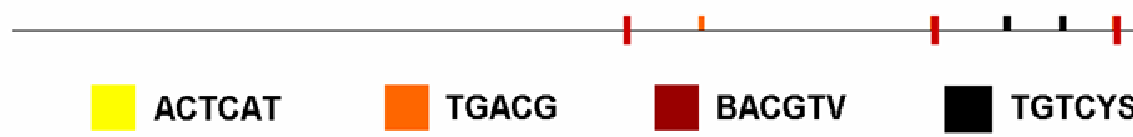

Supplemental Figure 1: Analysis of group II GH3 promoters from diverse plant species. A) Phylogenetic tree of group II GH3s from Arabidopsis thaliana (At), Nicotiana tabaccum (Nt) and Glycine $\max (\mathrm{Gm})$. Classification of GH3s has been adapted from Staswick et al. (2005). B) Closely related AtGH3.3 genes share a similar promoter (Pro) organisation in their $-1000 \mathrm{bp}$ region with respect to frequency and distribution of postulated bZIP (ACTCAT; TGACG; BACGTV) and ARF (TGTCYS) related binding sites. Consensus sequences are colour coded. 
A

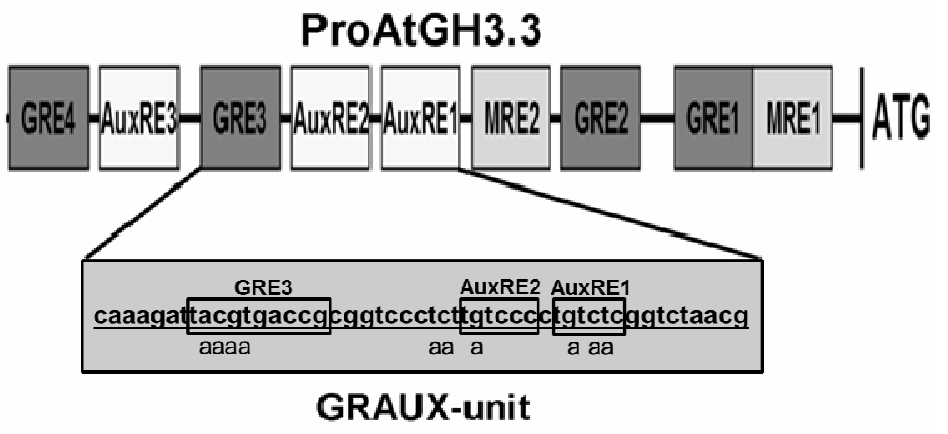

B

Group S1 AtbZIPs

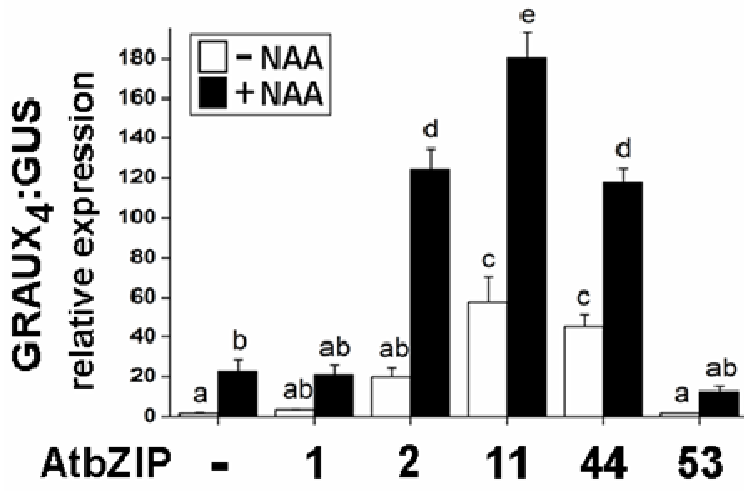

Group C AtbZIPs

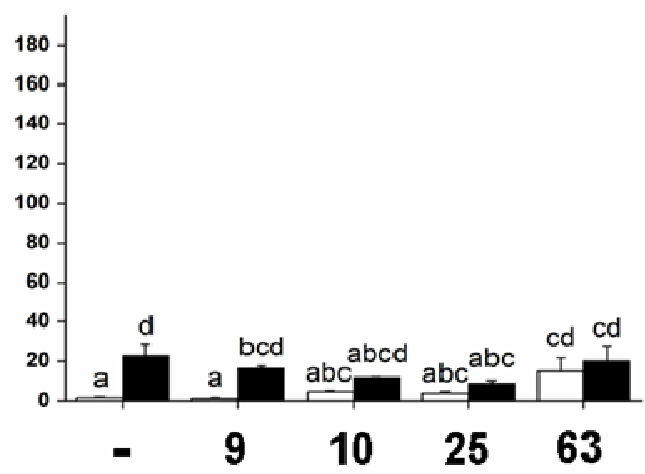

Supplemental Figure 2: Group S1 AtbZIP2, -11 and -44 strongly promote transcription of the AtGH3.3 derived GRAUX-module. A) Schematic view of promoter context, sequence and GRE and AuxRE ciselements of the AtGH3.3 promoter derived GRAUX-unit. B) Impact of group C/S1 AtbZIPs on GRAUX-

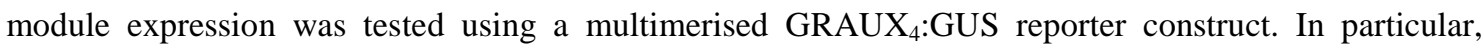
group S1 AtbZIP2, -11 and -44 co-expression enhance reporter gene expression in the absence (white bars) and presence (black bars) of exogenously applied auxin (0.25 $\mu \mathrm{M}$ NAA for 16h). Given are mean values $( \pm \mathrm{SD})$ from 3 independent experiments. A Pro35S:NAN construct was used for normalisation (Ehlert et al., 2006). Different letters indicate significant differences between constructs and treatments defined by one-way ANOVA, followed by Fisher posthoc test $(\mathrm{p} \leq 0.05)$. 
A

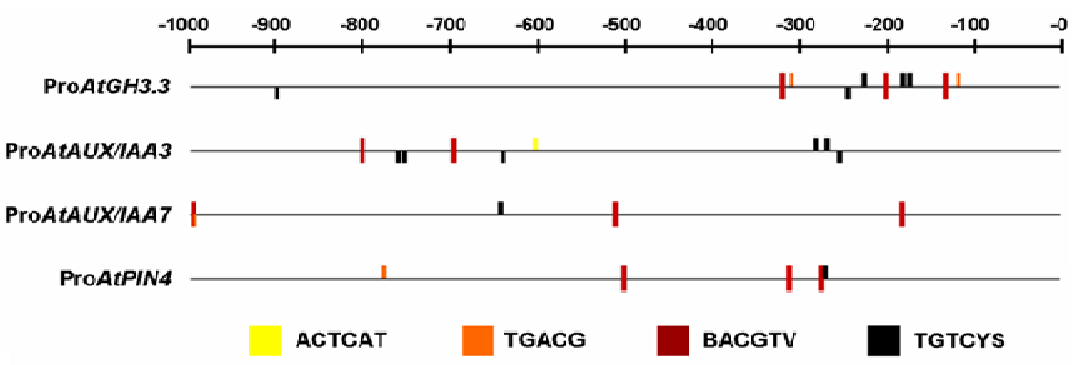

B

Group S1 AtbZIPs

Group C AtbZIPs
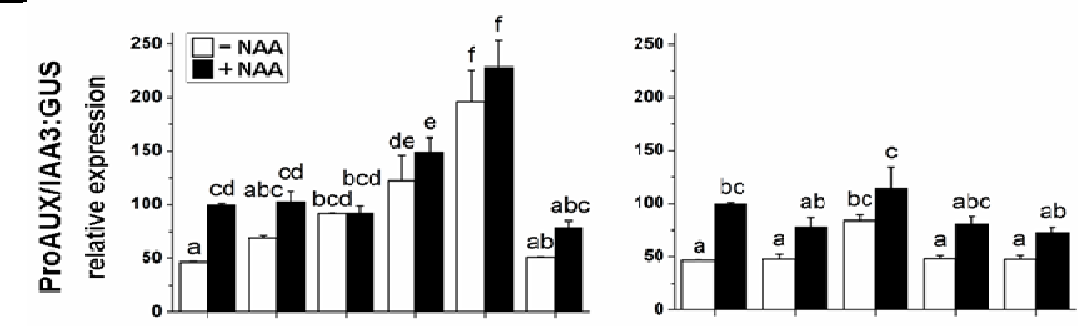

C
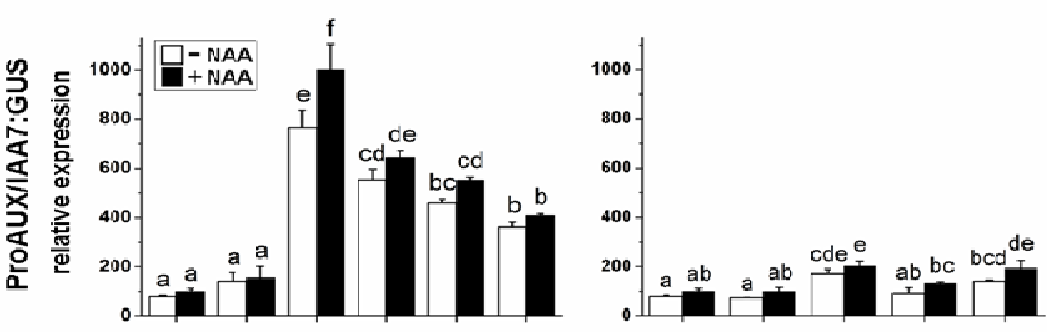

D
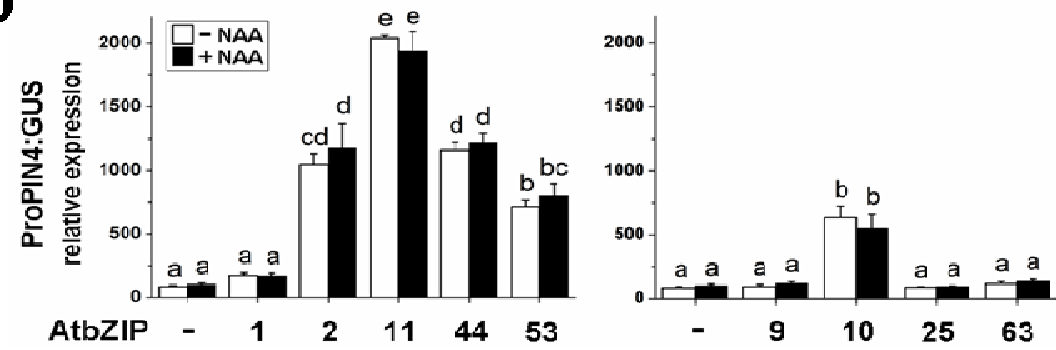

Supplemental Figure 3: Particular group C/S1 AtbZIPs regulate auxin-responsive promoters. A) The $1000 \mathrm{bp}$ promoter regions from auxin inducible AtGH3.3, AtAUX/IAA3, AtAUX/IAA7 and AtPIN4 genes display several AtbZIP- (ACTCAT; TGACG; BACGTV) and ARF-TF (TGTCYS) specific binding sites. Consensus sequences are colour coded. Arabidopsis protoplasts have been co-transformed with promoter:GUS constructs of either (B) AtAux/IAA3, (C) AtAux/IAA7 and (D) AtPIN4 and the AtbZIP effector constructs indicated (left: group S1, right: group C). Reporter gene expression has been quantified without (white bars) or with addition of $0.25 \mu \mathrm{M}$ NAA (black bars) for $16 \mathrm{~h}$. Given are mean values $( \pm \mathrm{SD}$ ) from 3 independent experiments with each of 2 replicates relative to the auxin induction of the corresponding promoter:GUS construct (100\%). A Pro35S:NAN construct is used for normalisation. Statistical relevant differences are determined by one-way ANOVA and Fisher posthoc test $(\mathrm{p} \leq 0.05)$ and are indicated by different letters. 


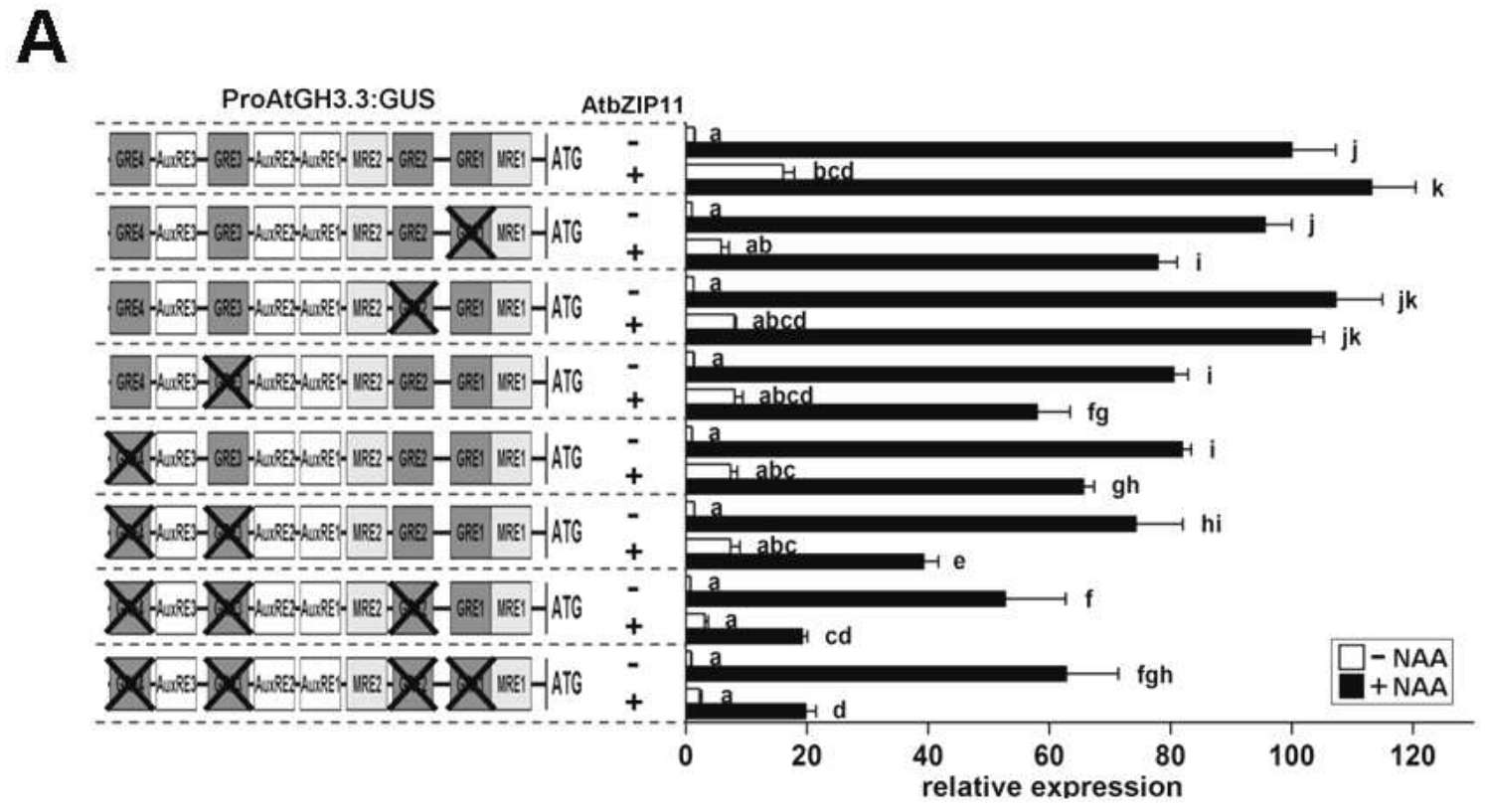

B

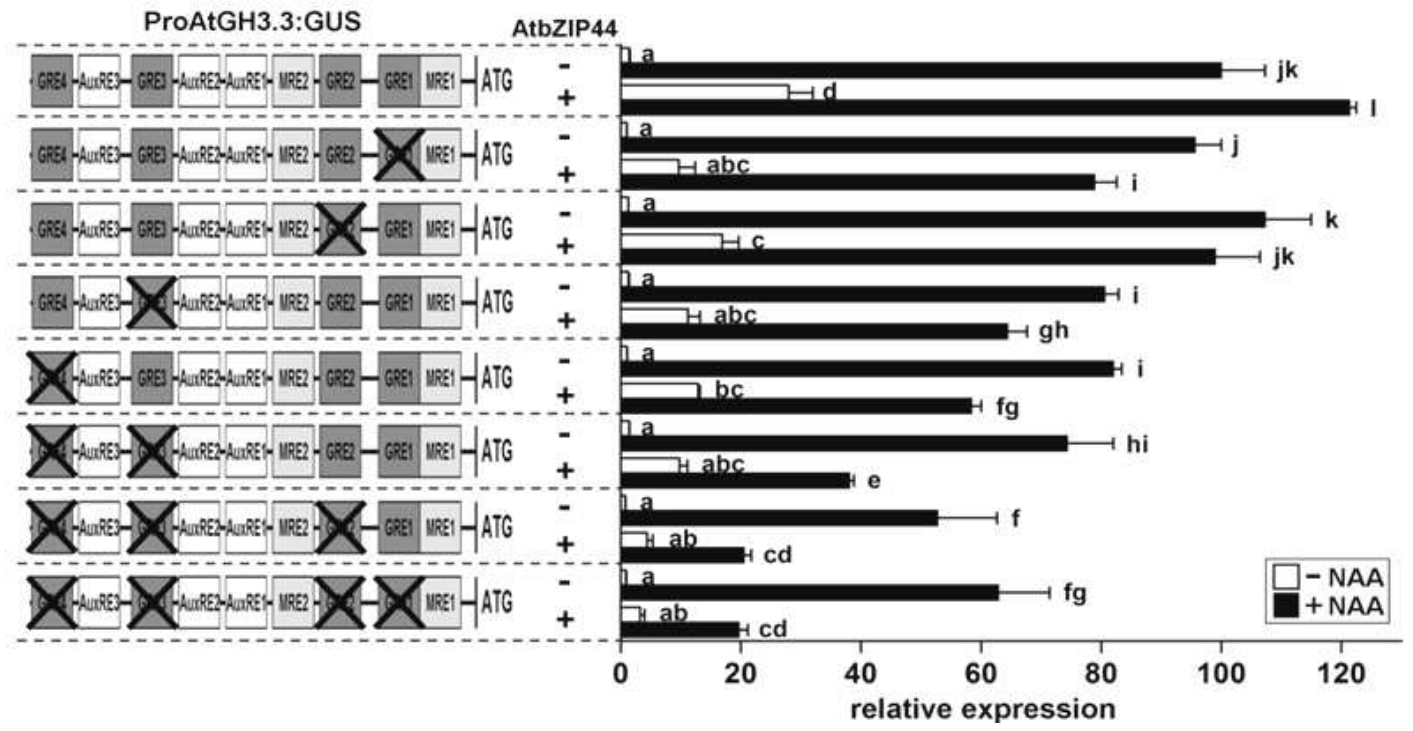

Supplemental Figure 4: Group S1 AtbZIPs regulate the AtGH3.3 promoter via GRE cis-elements. AtbZIP11 (A) and AtbZIP44 (B) promote ProAtGH3.3 driven reporter gene expression in the absence (white bars) and presence (black bars) of exogenously applied auxin ( $0.25 \mu \mathrm{M}$ NAA for $16 \mathrm{~h}$ ). With increasing number of mutated GRE cis-elements in the AtGH3.3 promoter, the contributory effect of AtbZIP TFs on reporter gene expression decreases. Given are mean values $( \pm \mathrm{SD})$. Distinct letters denote significant differences between constructs and treatments defined by one-way ANOVA and subsequent Fisher posthoc test $(\mathrm{p} \leq 0.05)$. 
A

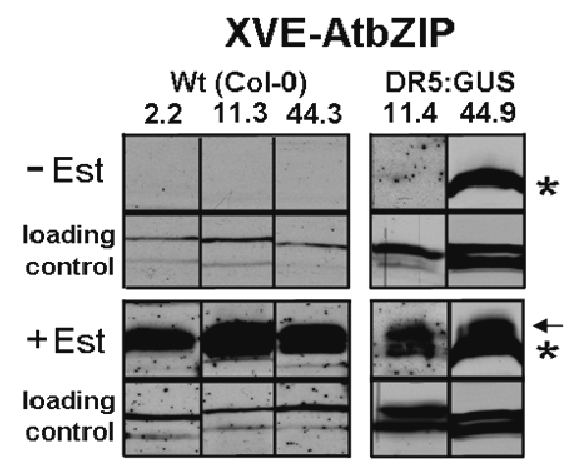

B WT (Col-0)

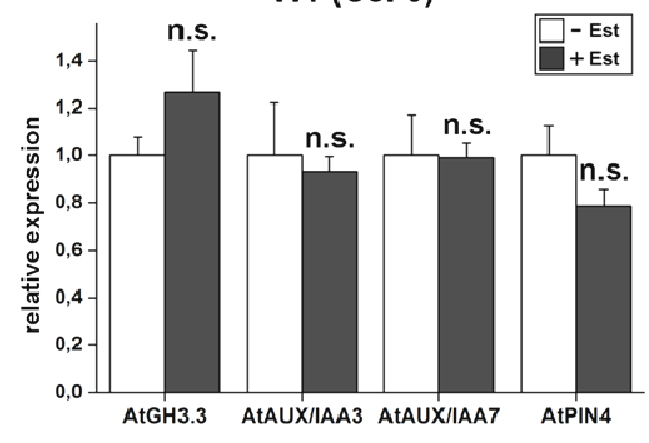

C

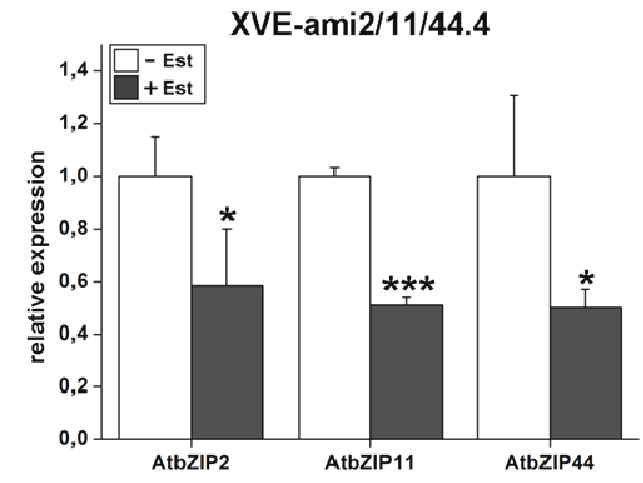

Supplemental Figure 5: The XVE system enables controlled AtbZIP expression by low Est concentrations, which do not unspecifically alter gene expression in WT plants. A) Immuno-detection of HA-tagged AtbZIP proteins detected in the uninduced (-Est) and Est-treated (+ $5 \mu \mathrm{M}$ Est for $24 \mathrm{~h}$ ) transgenic XVE-AtbZIP2, -11 and -44 plant lines indicated. Unspecific background signals are labelled by *. B) QRT-PCR analysis of AtGH3.3, AtAUX/IAA3, AtAUX/IAA7 and AtPIN4 transcript abundance in mock (DMSO) and Est-treated (10 $\mu \mathrm{M}$ for $24 \mathrm{~h})$ Arabidopsis WT plants. C) XVE plants harbouring an Est-inducible amiRNA construct targeting AtbZIP2, -11 and -44 transcripts (XVE-amiRNA2/11/44, line 4) show a reduced bZIP specific transcript abundance after Est induction ( $7 \mu \mathrm{M}$ for $20 \mathrm{~h}$ ) compared to mock (DMSO) treated plants. QRT-PCR data presented in (B) and (C) were obtained from 3 replicates consisting each of 3 individual plant pools. Given are mean expression levels $( \pm$ SEM) relative to uninduced conditions. Significant differences are defined by Students T-Test and illustrated by asterisks $(\mathrm{p} \leq 0.05=* ; \mathrm{p} \leq 0.01=* * ; \mathrm{p} \leq 0.001=* * *)$. 
A

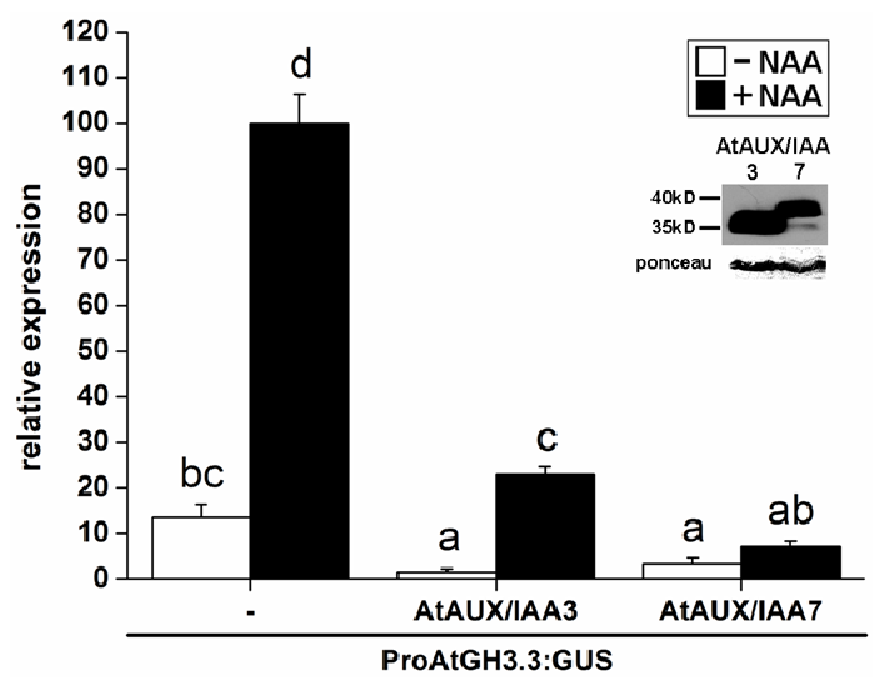

B

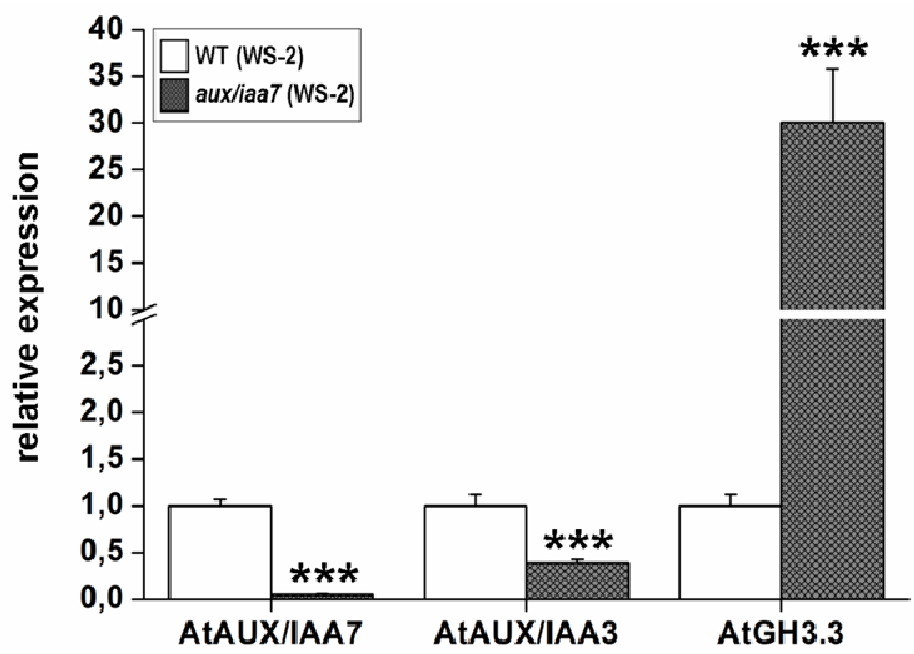

Supplemental Figure 6: $A t G H 3.3$ expression is negatively regulated by AtAUX/IAA3 and AtAUX/IAA7. A) Mesophyll protoplasts were co-transfected with the ProAtGH3.3 reporter and Pro35S:AtAux/IAA3 or Pro35S:AtAux/IAA7 effector constructs. Both effectors significantly repress ProAtGH3.3:GUS expression in the absence (white bars) or presence (black bars; $0.25 \mu \mathrm{M}$ NAA for $16 \mathrm{~h}$ ) of supplemented auxin. Given are mean expression levels ( \pm SD). Expression of HA-tagged effectors was monitored by immuno-detection (inset). Significant differences between treatments and applied constructs were determined by one-way ANOVA and Fisher posthoc test $(\mathrm{p} \leq 0.05)$ and visualised by varying letters. B) Q-RT-PCR analysis of AtAux/IAA7, AtAux/IAA3 and AtGH3.3 expression in leaves from Arabidopsis WT (white bars) and aux/iaa7 mutant (gray, hatched bars) plants. Given is the mean relative expression $( \pm$ SEM) referred to WT from 3 replicates from each of 3 individual plants. Significant differences between gene expression levels in WT and mutant plants are defined by Students T-Test and marked with asterisks $(\mathrm{p} \leq 0.05=* ; \mathrm{p} \leq 0.01=* * ; \mathrm{p} \leq 0.001=* * *)$. 


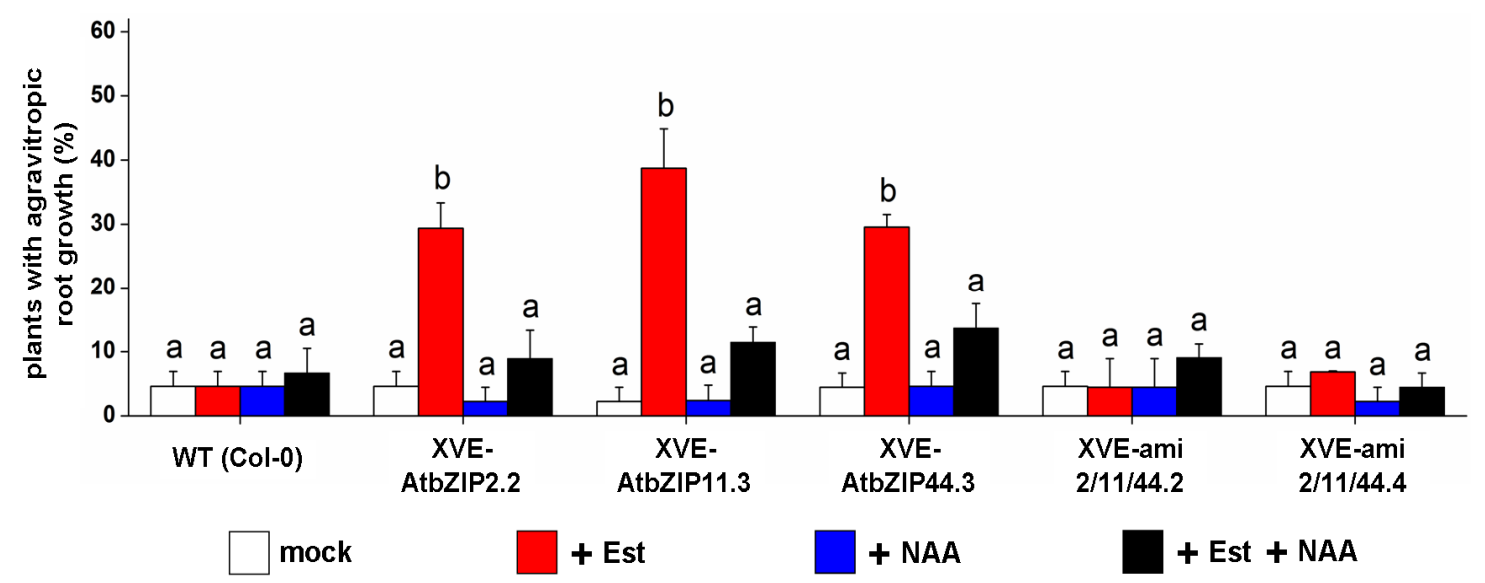

Supplemental Figure 7: Expression of AtbZIP2, -11 or -44 leads to agravitropic root growth. Arabidopsis WT and transgenic XVE-AtbZIP2, -11 and -44 as well as XVE-ami2/11/44 plant lines were cultivated for 7 days on MS medium supplemented with or without $10 \mu \mathrm{M}$ Est and/or $0.25 \mu \mathrm{M}$ NAA. Estinduced group S1 AtbZIP2, -11 or -44 expression causes agravitropic root growth which is partially abrogated by additional auxin treatment. Presented is the mean plant number displaying agravitropic root growth $( \pm$ SEM) from a total of 44 individual plants tested. Applied hormone treatments are illustrated by differently coloured bars, following the colour code from Figure 5. Significant differences between treatments are determined by one-way ANOVA and Fisher posthoc test $(\mathrm{p} \leq 0.05)$ and labelled with different letters. 
Supplemental Table 1: List of oligonucleotide sequences used in this study.

\begin{tabular}{|c|c|c|}
\hline category & Primer & sequence $\left(5^{\prime}-3^{\prime}\right)$ \\
\hline \multirow{32}{*}{ 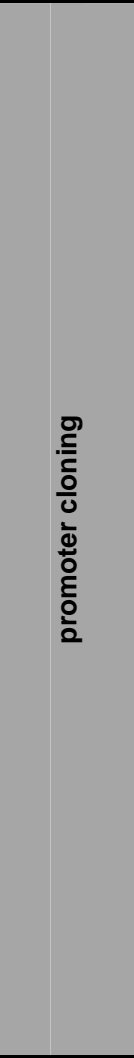 } & pAtGH3.3 1-1700 for & AAAAATCTAGAGTGCTGAATATTTT \\
\hline & pAtGH3.3 1-1700 rev & AAAAACCATGGGATTAAAATGGTAT \\
\hline & pAtGH3.3 300-1700 for & AGGCAGAGTCACAAGCCTAATATTAGGGAACCGCGTGGTAC \\
\hline & pAtGH3.3 300-1700 rev & GTACCACGCGGTTCCCTAATATTAGGCTTGTGACTCTGCCT \\
\hline & pAtGH3.3 AuxRE1 mut for & CGCGGTCCCTCTTGTCCCCTATAACGGTCTAACGATAACAA \\
\hline & pAtGH3.3 AuxRE1 mut rev & TTGTTATCGTTAGACCGTTATAGGGGACAAGAGGGACCGCG \\
\hline & pAtGH3.3 AuxRE2 mut for & CGTGACCGCGGTCCCAATTATCCCCTGTCTCGGTC \\
\hline & pAtGH3.3 AuxRE2 mut rev & GACCGAGACAGGGGATAATTGGGACCGCGGTCACG \\
\hline & pAtGH3.3 AuxRE3 mut for & GGCGCAGACATATCAGTCCCACATATAAGCCCAAAACTAGCCAAA \\
\hline & pAtGH3.3 AuxRE3 mut rev & TTTGGCTAGTTTTGGGCTTATATGTGGGACTGATATGTCTGCGCC \\
\hline & pAtGH3.3 GRE1 mut for & TGTCGACGTGGAATTTGGCTCCCTTTGGTTTTCTCCTTCTTGCC \\
\hline & pAtGH3.3 GRE1 mut rev & GGCAAGAAGGAGAAAACCAAAGGGAGCCAAATTCCACGTCGACA \\
\hline & pAtGH3.3 GRE2 mut for & AAACCGAGCCCACTTTTTATGTCGAAAAGGAATTTGGGCTGACGTTG \\
\hline & pAtGH3.3 GRE2 mut rev & CAACGTCAGCCAAATTCCTTTTCGACATAAAAGTGGGCTCGGTTT \\
\hline & pAtGH3.3 GRE3 mut for & GTCTGCCCAAAACTAGCCAAAGATTAAAAGACCGCGGTCCCTC \\
\hline & pAtGH3.3 GRE3 mut rev & GAGGGACCGCGGTCTTTTAATCTTTGGCTAGTTTTGGGCAGAC \\
\hline & pAtGH3.3 GRE4 mut for & CAATAAATTGCCCAATCAAAGTAACATGCCAAAAAGGCGCAGACATATCAGT \\
\hline & pAtGH3.3 GRE4 mut rev & ACTGATATGTCTGCGCCTTTTTGGCATGTTACTTTGATTGGGCAATTTATTG \\
\hline & pAtGH3.3 MRE1 mut for & CGACGTGGAATTTGGCTGACGTTAAATTTCTCCTTCTTGCCACTATAAA \\
\hline & pAtGH3.3 MRE1 mut rev & TTTATAGTGGCAAGAAGGAGAAATTTAACGTCAGCCAAATTCCACGTCG \\
\hline & pAtGH3.3 MRE2 mut for & CCTCTTGTCCCCTGTCTCGGTCTAACGATTTTAGACCGAGCCCACTTTTT \\
\hline & pAtGH3.3 MRE2 mut rev & AAAAGTGGGCTCGGTCTAAAATCGTTAGACCGAGACAGGGGACAAGAGG \\
\hline & PGRAUX for & AATTCACTAGTCAAAGATTACGTGACCGCGGTCCCTCTTGTCCCCTGTCTCGGTCTAACGT \\
\hline & pGRAUX rev & CTAGACGTTAGACCGAGACAGGGGACAAGAGGGACCGCGGTCACGTAATCTTTGACTAGTG \\
\hline & pGRAUX GRE mut for & AATTCACTAGTCAAAGATTAAAAGACCGCGGTCCCTCTTGTCCCCTGTCTCGGTCTAACGT \\
\hline & pGRAUX GRE mut rev & CTAGACGTTAGACCGAGACAGGGGACAAGAGGGACCGCGGTCTTTTAATCTTTTGACTAGTG \\
\hline & pAtAUX/IAA3 for & AAAAAAAATCTAGAGGAGGAATTTAATTAGGTTTTAATCCGACATATAAG \\
\hline & pAtAUX/IAA3 rev & AAAAAAAACCATGGTTCTTCAAGAATTGCAGGAGAAGATAAAAAG \\
\hline & pAtAUX/IAA7 for & AAAAAAAAACTAGTTTCACTCGATTGGTTGCGCATCAAATG \\
\hline & pAtAUX/IAA7 rev & AAAAAAAACCATGGTTACTTGTAATAGATTAGAAATATTGTTTCTCTCTCTG \\
\hline & pAtPIN4 for & AAAAACTGCAGGTTTATCTACATCACAGGTCTGGTAGATAAAG \\
\hline & pAtPIN4 rev & TTTTTCCATGGTTTTTCCGGTGGGTTTTGGAGTTTAG \\
\hline \multirow{16}{*}{$\begin{array}{l}\frac{\alpha}{0} \\
\frac{n}{\dot{r}} \\
\frac{\mathbf{r}}{\sigma}\end{array}$} & AtGH3.3 QP for & CATCACAGAGTTCCTCACAAGC \\
\hline & AtGH3.3 QP rev & GTCGGTCCATGTCTTCATCA \\
\hline & AtAUX/IAA3 QP for & AAAGGCTCAGATTGTTGGATGGC \\
\hline & AtAUX/IAA3 QP rev & TGACCCTCATGCTCAGATTCATTC \\
\hline & AtAUX/IAA7 QP for & AAGCTACCAGGATCTTTCTGATGC \\
\hline & AtAUX/IAA7 QP rev & ATTCCTTGTGCTCCATAGTTTCCC \\
\hline & AtPIN4 QP for & TTGTCTCTGATCAACCTCGAAA \\
\hline & AtPIN4 QP rev & ATCAAGACCGCCGATATCAT \\
\hline & AtbZIP2 RT for & TGATCGGAAACTGATGACTCC \\
\hline & AtbZIP2 RT rev & GAGCAGATTTTACCGTGAGC \\
\hline & AtbZIP11 RT for & CGATTCAAACGTCGTCAGG \\
\hline & AtbZIP11 RT rev & TCCGTTTACGTTTCCTCTGC \\
\hline & AtbZIP44 RT for & CATCTACGTAAAGAAAACGCTCAG \\
\hline & AtbZIP44 RT rev & CCGGTCTCCATACCGAATC \\
\hline & UBQ5 RT for & GACGCTTCATCTCGTCC \\
\hline & UBQ5 RT rev & GTAAACGTAGGTGAGTCCA \\
\hline \multirow{6}{*}{$\frac{n}{\frac{n}{v}}$} & AtGH3.3 gene ChIP for & CCCCATCACAGAGTTCCTCACAAGG \\
\hline & AtGH3.3 gene ChIP rev & TGGCATCAACTTCCTTTCACCAGC \\
\hline & AtGH3.3 prom. ChIP for & TGCCAACGTGGCGCAGACATATCAGTCCC \\
\hline & AtGH3.3 prom. ChIP rev & CAAGAAGGAGAAAACCAACGTCAGCC \\
\hline & AtActin8 ChIP for & GGTTTTCCCCAGTGTTGTTG \\
\hline & AtActin8 ChIP rev & CTCCATGTCATCCCAGTTGC \\
\hline \multirow{6}{*}{ 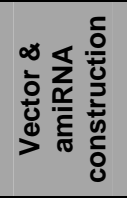 } & amiRNA AtbZIP2/11/44 I & GATTCGTTAAGAGATTGGAGACTTCTCTCTTTTGTATTCC \\
\hline & amiRNA AtbZIP2/11/44 II & GAAGTCTCCAATCTCTTAACGAATCAAAGAGAATCAATGA \\
\hline & amiRNA AtbZIP2/11/44 III & GACGTCTCCAATCACTTAACGAATCACAGGTCGTGATATG \\
\hline & amiRNA AtbZIP2/11/44 IV & GATTCGTTAAGTGATTGGAGACGTCTACATATATATTCCT \\
\hline & pMDC7-HA for & AAAACTCGAGATGGCATACCCATACGACGTTCCGG \\
\hline & pMDC7-HA rev & AAAACTCGAGATCTGCCTAGAGATATCTGCATAGTCCGGG \\
\hline
\end{tabular}




\section{References}

Abel, S., Nguyen, M.D., and Theologis, A. (1995). The PS-IAA4/5-like family of early auxin-inducible mRNAs in Arabidopsis thaliana. J Mol Biol 251, 533-549.

Abel, S., and Theologis, A. (2010). Odyssey of Auxin. Cold Spring Harb Perspect Biol.

Alonso, R., Onate-Sanchez, L., Weltmeier, F., Ehlert, A., Diaz, I., Dietrich, K., Vicente-Carbajosa, J., and Dröge-Laser, W. (2009). A pivotal role of the basic leucine zipper transcription factor bZIP53 in the regulation of Arabidopsis seed maturation gene expression based on heterodimerization and protein complex formation. Plant Cell. 21, 1747-1761.

Baena-Gonzalez, E., Rolland, F., Thevelein, J.M., and Sheen, J. (2007). A central integrator of transcription networks in plant stress and energy signalling. Nature 448, 938-942.

Blilou, I., Xu, J., Wildwater, M., Willemsen, V., Paponov, I., Friml, J., Heidstra, R., Aida, M., Palme, K., and Scheres, B. (2005). The PIN auxin efflux facilitator network controls growth and patterning in Arabidopsis roots. Nature 433, 39-44.

Böttner, S., Iven, T., Carsjens, C.S., and Dröge-Laser, W. (2009). Nuclear accumulation of the ankyrin repeat protein ANK1 enhances the auxin-mediated transcription accomplished by the bZIP transcription factors BZI-1 and BZI-2. Plant J 58, 914-926.

Dharmasiri, N., Dharmasiri, S., and Estelle, M. (2005). The F-box protein TIR1 is an auxin receptor. Nature. 435, 441-445.

Dietrich, K., Weltmeier, F., Ehlert, A., Weiste, C., Stahl, M., Harter, K., and Dröge-Laser, W. (2011). Heterodimers of the Arabidopsis Transcription Factors bZIP1 and bZIP53 Reprogram Amino Acid Metabolism during Low Energy Stress. Plant Cell.

Dubos, C., Stracke, R., Grotewold, E., Weisshaar, B., Martin, C., and Lepiniec, L. (2010). MYB transcription factors in Arabidopsis. Trends Plant Sci 15, 573-581.

Ehlert, A., Weltmeier, F., Wang, X., Mayer, C.S., Smeekens, S., Vicente-Carbajosa, J., and DrögeLaser, W. (2006). Two-hybrid protein-protein interaction analysis in Arabidopsis protoplasts: establishment of a heterodimerization map of group C and group S bZIP transcription factors. Plant $\mathrm{J}$ 46, 890-900.

Gil, P., and Green, P.J. (1996). Multiple regions of the Arabidopsis SAUR-AC1 gene control transcript abundance: the 3' untranslated region functions as an mRNA instability determinant. Embo $\mathrm{J} 15$, 1678-1686.

Gray, W.M., and Estelle, I. (2000). Function of the ubiquitin-proteasome pathway in auxin response. Trends Biochem. Sci. 25, 133-138.

Guilfoyle, T.J., and Hagen, G. (2007). Auxin response factors. Curr Opin Plant Biol. 10, 453-460. Epub 2007 Sep 2027.

Hanson, J., Hanssen, M., Wiese, A., Hendriks, M.M.W.B., and S, S. (2008). The sucrose regulated transcription factor bZIP11 affects amino acid metabolism by regulating the expression of Asparagin Synthase1 and Proline Dehydrogenase2. Plant J. 53, 935-949.

Heinekamp, T., Strathmann, A., Kuhlmann, M., Froissard, M., Muller, A., Perrot-Rechenmann, C., and Dröge-Laser, W. (2004). The tobacco bZIP transcription factor BZI-1 binds the GH3 promoter in vivo and modulates auxin-induced transcription. Plant J 38, 298-309.

Hiratsu, K., Matsui, K., Koyama, T., and Ohme-Takagi, M. (2003). Dominant repression of target genes by chimeric repressors that include the EAR motif, a repression domain, in Arabidopsis. Plant J. 34, 733-739.

Iven, T., Strathmann, A., Bottner, S., Zwafink, T., Heinekamp, T., Guivarc'h, A., Roitsch, T., and Dröge-Laser, W. (2010). Homo- and heterodimers of tobacco bZIP proteins counteract as positive or negative regulators of transcription during pollen development. Plant J 63, 155-166.

Jakoby, M., Weisshaar, B., Dröge-Laser, W., Vicente-Carbajosa, J., Tiedemann, J., Kroj, T., and Parcy, F. (2002). bZIP transcription factors in Arabidopsis. Trends Plant Sci 7, 106-111.

Kaminaka, H., Nake, C., Epple, P., Dittgen, J., Schütze, K., Chaban, C., Holt, B.F., 3rd, Merkle, T., Schäfer, E., Harter, K., and Dangl, J.L. (2006). bZIP10-LSD1 antagonism modulates basal defense and cell death in Arabidopsis following infection. EMBO J. 25, 4400-4411. 
Kepinski, S., and Leyser, O. (2005). The Arabidopsis F-box protein TIR1 is an auxin receptor. Nature. 435, 446-451.

Kirby, J., and Kavanagh, T.A. (2002). NAN fusions: a synthetic sialidase reporter gene as a sensitive and versatile partner for GUS. Plant J 32, 391-400.

Liscum, E., and Reed, J.W. (2002). Genetics of Aux/IAA and ARF action in plant growth and development. Plant Mol Biol 49, 387-400.

Liu, Z.B., Hagen, G., and Guilfoyle, T.J. (1997a). A G-box-binding protein from soybean binds to the E1 auxin-response element in the soybean GH3 promoter and contains a proline-rich repression domain. Plant Physiol. 115, 397-407.

Liu, Z.B., Hagen, G., and Guilfoyle, T.J. (1997b). A G-Box-Binding Protein from Soybean Binds to the E1 Auxin-Response Element in the Soybean GH3 Promoter and Contains a Proline-Rich Repression Domain. Plant Physiol 115, 397-407.

Liu, Z.B., Ulmasov, T., Shi, X., Hagen, G., and Guilfoyle, T.J. (1994). Soybean GH3 promoter contains multiple auxin-inducible elements. Plant Cell 6, 645-657.

Moubayidin, L., Perilli, S., Dello Ioio, R., Di Mambro, R., Costantino, P., and Sabatini, S. (2010). The rate of cell differentiation controls the Arabidopsis root meristem growth phase. Curr Biol 20, 1138-1143.

Moulia, B., and Fournier, M. (2009). The power and control of gravitropic movements in plants: a biomechanical and systems biology view. J Exp Bot 60, 461-486.

Müller, A., Guan, C., Galweiler, L., Tanzler, P., Huijser, P., Marchant, A., Parry, G., Bennett, M., Wisman, E., and Palme, K. (1998). AtPIN2 defines a locus of Arabidopsis for root gravitropism control. Embo J 17, 6903-6911.

Murashige, T., and Skoog, F. (1962). A revised medium for rapid growth and bioassays with tobacco tissue cultures. Physiol. Plant. . 15, 473-497.

Navarro, L., Dunoyer, P., Jay, F., Arnold, B., Dharmasiri, N., Estelle, M., Voinnet, O., and Jones, J.D. (2006). A plant miRNA contributes to antibacterial resistance by repressing auxin signaling. Science. 312, 436-439.

Obertello, M., Krouk, G., Katari, M.S., Runko, S.J., and Coruzzi, G.M. (2010). Modeling the global effect of the basic-leucine zipper transcription factor 1 (bZIP1) on nitrogen and light regulation in Arabidopsis. BMC Syst Biol 4, 111.

Overvoorde, P., Fukaki, H., and Beeckman, T. (2010). Auxin control of root development. Cold Spring Harb Perspect Biol 2, a001537.

Paponov, I.A., Paponov, M., Teale, W., Menges, M., Chakrabortee, S., Murray, J.A., and Palme, K. (2008). Comprehensive transcriptome analysis of auxin responses in Arabidopsis. Mol Plant 1, 321337.

Pauwels, L., Barbero, G.F., Geerinck, J., Tilleman, S., Grunewald, W., Perez, A.C., Chico, J.M., Bossche, R.V., Sewell, J., Gil, E., et al. (2010). NINJA connects the co-repressor TOPLESS to jasmonate signalling. Nature 464, 788-791.

Robert-Seilaniantz, A., Navarro, L., Bari, R., and Jones, J.D. (2007). Pathological hormone imbalances. Curr Opin Plant Biol 10, 372-379.

Rook, F., Gerrits, N., Kortstee, A., van Kampen, M., Borrias, M., Weisbeek, P., and Smeekens, S. (1998a). Sucrose-specific signalling represses translation of the Arabidopsis ATB2 bZIP transcription factor gene. Plant J 15, 253-263.

Rook, F., Weisbeek, P., and Smeekens, S. (1998b). The light-regulated Arabidopsis bZIP transcription factor gene ATB2 encodes a protein with an unusually long leucine zipper domain. Plant Mol Biol 37, 171-178.

Rushton, P.J., Reinstadler, A., Lipka, V., Lippok, B., and Somssich, I.E. (2002). Synthetic plant promoters containing defined regulatory elements provide novel insights into pathogen- and woundinduced signaling. Plant Cell 14, 749-762.

Sambrook, J., Fritsch, E.F., and Maniatis, T. (1989). Molecular Cloning: a Laboratory Manual (New York: Cold Spring Harbour Laboratory Press).

Scarpella, E., Barkoulas, M., and Tsiantis, M. (2010). Control of leaf and vein development by auxin. Cold Spring Harb Perspect Biol 2, a001511. 
Schwab, R., Ossowski, S., Riester, M., Warthmann, N., and Weigel, D. (2006). Highly specific gene silencing by artificial microRNAs in Arabidopsis. Plant Cell 18, 1121-1133.

Sheen, J. (2001). Signal transduction in maize and Arabidopsis mesophyll protoplasts. Plant Physiol 127, 1466-1475.

Shin, R., Burch, A.Y., Huppert, K.A., Tiwari, S.B., Murphy, A.S., Guilfoyle, T.J., and Schachtman, D.P. (2007). The Arabidopsis transcription factor MYB77 modulates auxin signal transduction. Plant Cell 19, 2440-2453.

Siberil, Y., Doireau, P., and Gantet, P. (2001). Plant bZIP G-box binding factors. Modular structure and activation mechanisms. Eur J Biochem 268, 5655-5666.

Staswick, P. (2009). Plant hormone conjugation: a signal decision. Plant Signal Behav 4, 757-759.

Staswick, P.E., Serban, B., Rowe, M., Tiryaki, I., Maldonado, M.T., Maldonado, M.C., and Suza, W. (2005). Characterization of an Arabidopsis enzyme family that conjugates amino acids to indole3-acetic acid. Plant Cell 17, 616-627.

Staswick, P.E., Tiryaki, I., and Rowe, M.L. (2002). Jasmonate response locus JAR1 and several related Arabidopsis genes encode enzymes of the firefly luciferase superfamily that show activity on jasmonic, salicylic, and indole-3-acetic acids in an assay for adenylation. Plant Cell 14, 1405-1415.

Strathmann, A., Kuhlmann, M., Heinekamp, T., and Dröge-Laser, W. (2001). BZI-1 specifically heterodimerises with the tobacco bZIP transcription factors BZI-2, BZI-3/TBZF and BZI-4, and is functionally involved in flower development. Plant J 28, 397-408.

Swaminathan, K., Peterson, K., and Jack, T. (2008). The plant B3 superfamily. Trends Plant Sci 13, 647-655.

Tiwari, S.B., Hagen, G., and Guilfoyle, T. (2003). The roles of auxin response factor domains in auxinresponsive transcription. Plant Cell 15, 533-543.

Ulmasov, T., Hagen, G., and Guilfoyle, T.J. (1997). ARF1, a transcription factor that binds to auxin response elements. Science 276, 1865-1868.

Ulmasov, T., Hagen, G., and Guilfoyle, T.J. (1999a). Activation and repression of transcription by auxin-response factors. Proc. Natl. Acad. Sci. USA 96, 5844-5849.

Ulmasov, T., Hagen, G., and Guilfoyle, T.J. (1999b). Dimerization and DNA binding of auxin response factors. Plant Journal, The 19, 309-319.

Ulmasov, T., Liu, Z.B., Hagen, G., and Guilfoyle, T.J. (1995). Composite structure of auxin response elements. Plant Cell 7, 1611-1623.

Weigel, R., and Glazebrook, J. (2002). Arabidopsis: A Laboratory Manual (New York: Cold Spring Harbour Laboratory Press).

Weiste, C., Iven, T., Fischer, U., Onate-Sanchez, L., and Droge-Laser, W. (2007). In planta ORFeome analysis by large-scale over-expression of GATEWAY-compatible cDNA clones: screening of ERF transcription factors involved in abiotic stress defense. Plant J 52, 382-390.

Weltmeier, F., Ehlert, A., Mayer, C.S., Dietrich, K., Wang, X., Schütze, K., Alonso, R., Harter, K., Vicente-Carbajosa, J., and Dröge-Laser, W. (2006). Combinatorial control of Arabidopsis proline dehydrogenase transcription by specific heterodimerisation of bZIP transcription factors. Embo J 25, 3133-3143.

Weltmeier, F., Rahmani, F., Ehlert, A., Dietrich, K., Schütze, K., Wang, X., Chaban, C., Hanson, J., Teige, M., Harter, K., et al. (2009). Expression patterns within the Arabidopsis C/S1 bZIP transcription factor network: availability of heterodimerization partners controls gene expression during stress response and development. Plant Mol Biol 69, 107-119.

Wilson, A.K., Pickett, F.B., Turner, J.C., and Estelle, M. (1990). A dominant mutation in Arabidopsis confers resistance to auxin, ethylene and abscisic acid. Mol Gen Genet 222, 377-383.

Woodward, A.W., and Bartel, B. (2005). Auxin: regulation, action, and interaction. Ann Bot (Lond). 95, 707-735. Epub 2005 Mar 2004.

Zhao, Y. (2010). Auxin biosynthesis and its role in plant development. Annu Rev Plant Biol 61, 49-64.

Zuo, J., Niu, Q.W., and Chua, N.H. (2000). Technical advance: An estrogen receptor-based transactivator XVE mediates highly inducible gene expression in transgenic plants. Plant J 24, 265273. 


\subsection{Chapter 3: Arabidopsis AtbZIP11-related transcription factors modulate auxin-mediated transcription by recruiting the histone acetylation machinery*}

* Parts of this manuscript are prepared for publication

Christoph Weiste ${ }^{1,2}$ and Wolfgang Dröge-Laser ${ }^{1, \#}$

${ }^{1}$ Julius-von-Sachs-Institut, Pharmazeutische Biologie, Julius-Maximilians-Universität Würzburg, Julius-von-Sachs-Platz 2, 97082 Würzburg, Germany

${ }^{2}$ Albrecht-von-Haller-Institut, Allgemeine und Entwicklungsphysiologie der Pflanze, Universität Göttingen, Untere Karspüle 2, 37073 Göttingen, Germany

\# To whom correspondence should be addressed:

Email: wolfgang.droege-laser@uni-wuerzburg.de

Tel +49-(0)931-31-80955; Fax: +49-(0)931-31-86182 


\section{Abstract}

In higher plants the hormone auxin orchestrates a diverse array of developmental and environmental responses mainly controlled via transcriptional regulation. In this respect auxin-induced genes are repressed by AUX/IAA proteins which are thought to recruit histone deacetylases to their cognate promoters in order to modify the chromatin into a highly-packed, inactive state. Although auxin-induced degradation of AUX/IAAs has been described to be a crucial step to de-repress these genes, a mechanism which leads to gene-specific histone acetylation remains elusive. In here, we assign Arabidopsis AtbZIP11-related, basic leucine Zipper transcription factors to recruit the SAGA-like acetylation machinery via their N-terminal activation domains. Pharmacological and reverse genetic approaches clearly define the impact of histone acetylation in auxininduced transcription. BZIP-mediated recruitment of the histone acetylation machinery and of RNA-Polymerase II has been confirmed by Chromatin-Immunoprecipitation (ChIP). In conclusion, we provide conclusive evidence for a novel regulatory mechanism which expands our understanding of auxin-regulated transcription.

\section{Introduction}

Various developmental and growth-related plant processes, such as embryogenesis, root and shoot architecture, organ patterning and vascular development are regulated by the plant hormone auxin (for review see Zhao, 2010; Woodward and Bartel, 2005). Moreover, responses to environmental signals such as tropic responses or pathogen defence are associated with this hormone (e. g. Navarro et al., 2006; Wang et al., 2007). To control these various responses, co-ordinated regulation of auxin-induced genes is required, which is primarily mediated by cis-acting AUXIN RESPONSIVE ELEMENTS (AuxREs) (Ulmasov et al., 1997; Ulmasov et al., 1999a,b), which are bound by AUXIN RESPONSE FACTORS (ARFs), members of the B3-type transcription factor (TF) family (Swaminathan et al., 2008; Waltner et al., 2005; Guilfoyle and Hagen, 2007). Via protein-protein interaction, ARF-mediated transcription is repressed by AUX/IAA proteins (Gray et al., 2001, Tiwari et al., 2004). 
These repressor proteins function due to their interaction with the Groucho/Tup type corepressor TOPLESS (TPL) which is thought to recruit histone deacetylases (HDACs) to its target promoters (Liu and Karmarkar, 2008; Szemenyei et al., 2008). Histone deacetylation is correlated with transcriptional inactive, tightly-packed chromatin (Pandey et al., 2002). In response to auxin perception, which is mediated by the F-box protein TIR1, Aux/IAA repressor proteins are polyubiquitinated by the $\mathrm{SCF}^{\mathrm{TIR} 1}$ complex and subsequently degraded by the 26S proteasome (Dharmasiri et al., 2005; Kepinski and Leyser, 2005). This regulatory mechanism based on de-repression enables rapid ARF-mediated gene activation (Guilfoyle and Hagen, 2007) and has been found to be a characteristic feature of several hormone signalling pathways (Pauwels et al., 2010). However, a complementary mechanism leading to activation of auxin-responsive genes via histone acetyltransferases (HATs) such as GCN5 (HAG1) has not been described, yet although GCN5 has been found in a mutant suppressor screen for the corepressor TPL (Szemenyei et al., 2008). GCN5 and the transcriptional adaptor protein AtADA2b (PRZ1) and AtADA2a physically interact and are, similar to their yeast orthologs, components of the Arabidopsis SAGA-like chromatin remodelling complex (for review see Servet et al., 2010, Anzola et al., 2010, Bhat et al., 2003, 2004). In fact, atgcn5 and atada2b mutants, in which SAGA complex formation should be disrupted, exhibit several, abnormal auxin related growth phenotypes, in particular severe dwarfism, loss of apical dominance, aberrant meristem function, abnormal root and leaf development and reduced petal and stamen growth (Vlachonasios et al., 2003, Bertrand et al., 2003; Long et al., 2006; Kornet and Scheres, 2009). In addition, AtADA2b was shown to be required for histone acetylation in response to auxin (Anzola et al., 2010). Consistently, the $a d a 2 b$ mutant plants are impaired in translating auxin signals into proper morphogenetic responses, as they form auxin induced callus-like root structures instead of lateral roots (Sieberer et al., 2003). AtADA2 proteins are capable to interact with DNA-binding TFs, suggesting that GCN5 or related HAT proteins can be recruited to specific promoters in SAGA-like complexes via AtADA2 mediated interactions (Servet et al., 2010). By now, two direct physical interactions between AtADA2 homologous proteins and transcriptional activators could be confirmed. Arabidopsis AtADA2b interacts with the AP2 transcription factor CBF1, which promotes expression of several cold-responsive genes (Stockinger et al., 2001; Mao et al., 2006), whereas 
ADA2 from maize binds the bZIP (basic leucine Zipper) factor OPAQUE2 (O2), which is involved in regulating seed storage genes during maize endosperm development (Bhat et al., 2004). With respect to O2, it has further been demonstrated, that histone acetylation of its target promoters and transcript accumulation of its target genes, correlates with promoter binding of O2, ADA2/GCN5 and RNA Polymerase II (RNPII) (Locatelli et al., 2009). This suggests that the activation potential of some TFs is facilitated by recruiting histone modifying enzymes.

In order to identify cis-regulatory elements and trans-binding transcription factors (TFs) which function as recruitment adapters of the histone acetylation machinery with respect to auxin-induced gene activation, we recently identified G-box related elements (GREs) and the closely homologous group S1 bZIP TFs AtbZIP11, -2 and -44 (Weiste et al., submitted). These TFs quantitatively modulate auxin-induced phenotypic responses and gene activation.

In this work we provide evidence that AtbZIP11-related TFs modulate expression of auxin-inducible genes via histone acetylation. Due to protein-protein interaction with AtADA2b, which is mediated by the bZIPs' N-terminal activation domain, they are able to recruit the Arabidopsis SAGA-complex. Chromatin Immunoprecipitation (ChIP) experiments confirm that bZIP-binding to the early auxin responsive AtGH3.3 promoter correlates with its enhanced, GCN5 specific histone H3K27 acetylation and activated RNPII binding. Altogether, these data provide a conclusive mechanistic model describing a bZIP-mediated recruitment of the histone acetylation machinery to activate auxin-induced transcription.

\section{Results}

\section{Pharmacological approaches reveal that auxin-regulated transcription is controlled by histone acetylation and deacetylation}

To confirm the assumption, that auxin-induced transcription is controlled by histone acetylation and deacetylation, we monitored the expression of the early auxin responsive AtGH3.3 and the moderately responding AtAUX/IAA3, AtAUX/IAA7 and AtPIN4 genes (Weiste et al., submitted) in the absence and presence of the broad- 
spectrum HDAC inhibitor Trichostatin A (TSA) (Yoshida et al., 1990; Lusser et al., 2001) or the GCN5 specific HAT inhibitor, y-butyrolacton (Biel et al., 2004). To ensure equal exposure of the plant cells to the additives, wild-type (wt) mesophyll protoplasts were prepared and pre-incubated for $3 \mathrm{hrs}$ in an isotonic solution supplemented with or without low concentrations of TSA $(0.8 \mu \mathrm{M})$ or y-butyrolacton $(0.25 \mathrm{mM})$, before they were subjected to an additional 3 hrs auxin treatment ( $2 \mu \mathrm{M}$ NAA). As demonstrated in Figure 1A, gene expression is enhanced by auxin and/or TSA, although gene-specific quantitative differences in induction could be observed. In particular, the moderately auxin-induced AtPIN4 gene was strongly de-repressed by TSA treatment (Figure 1A).

In Arabidopsis five HAT-genes, related to the yeast GCN5 have been described, termed

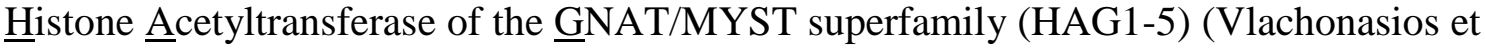
al., 2003; Benhamed et al., 2006; Benhamed et al., 2008; Pandey et al., 2002). In mammals, y-butyrolacton efficiently targets specific amino acids (aa) within the catalytic active site of the GCN5 enzyme. Since these aa residues are conserved in AtHAG1 (AtGCN5), AtHAG4 and AtHAG5 (Figure S1) the activity of these enzymes is likely affected by this HAT inhibitor.

Whereas y-butyrolacton had no effect on gene-induction in the absence of externally applied auxin, it significantly reduced auxin-induced transcription when both compounds were applied (Figure 1B). In contrast to the inhibitor driven expressional changes on the auxin-responsive genes tested, UBQ5 transcript abundance is largely unaffected by all treatments used (Figure S2). In summary, these pharmacological data clearly underline the involvement of histone acetylation and deacetylation in auxinregulated transcription. 
A

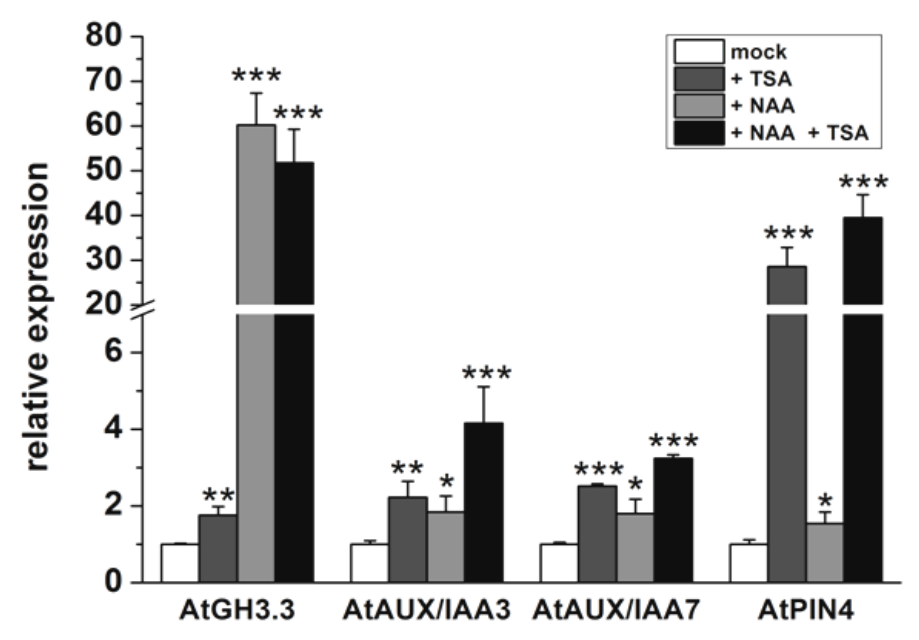

B

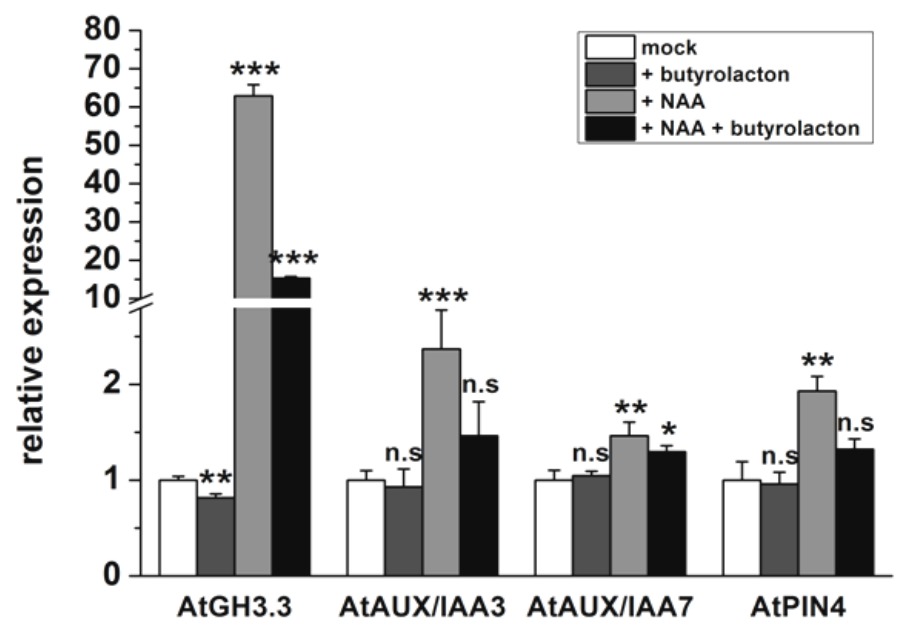

Figure 1: Auxin responsive gene expression in regulated by histone acetylation and deacetylation. Expression analysis of the auxin-responsive AtGH3.3, AtAUX/IAA3, AtAUX/IAA7 and AtPIN4 genes in the absence or presence of auxin and (A) the histone deacetylase (HDAC) inhibitor, Trichostatin A (TSA) or (B) the GCN5 histone acetyltransferase (HAT) inhibitor, y-butyrolacton. To ensure equal exposure to the additives, mesophyll protoplasts were prepared and incubated for 3 hours in an isotonic incubation buffer supplemented with or without $0.8 \mu \mathrm{M}$ TSA or $0.25 \mathrm{mM}$ y-butyrolacton before expression of auxin responsive genes was induced for further 3 hours by additional NAA $(2 \mu \mathrm{M})$ application. Each gene's expression was quantified by qRT-PCR and is presented as relative expression level compared to mock (DMSO) treated samples (white bars). Respective additive treatment, such as auxin (light gray), HAT/HDAC inhibitor (dark gray) or a combined auxin/inhibitor treatment (black) is visualized by individually coloured bars. Given are mean values $( \pm$ SEM) from three independent experiments with each of four technical replicates. Statistical significant differences compared to mock treated samples for each gene's expression was determined by Students T-Test and is illustrated by asterisks ( $\mathrm{p} \leq 0.05=* ; \mathrm{p}$ $\leq 0.01=* * ; \mathrm{p} \leq 0.001=* * *)$. 
AtbZIP11-related basic leucine Zipper transcription factors interact with ADA2 adapter proteins of the histone acetylation machinery

Recently, the group S1 bZIP factors AtbZIP11, -2 and -44 have been demonstrated to quantitatively modulate auxin-mediated transcription via GREs (Weiste et al., submitted). To disclose the mechanism how these TFs regulate transcription, we tested the hypothesis, whether these bZIPs are able to recruit the histone acetylation machinery to open-up packed chromatin. Whereas only a minor interaction between GCN5 and group C and S1 bZIPs (unpublished data) could be observed in a protoplast two-hybrid approach (P2H) (Ehlert et al., 2006), a significant protein interaction between AtADA2b and the closely related group S1 members AtbZIP2, -11 and -44 and group C AtbZIP10 and -25 could be identified (Figure $\mathbf{2 A}$ ). Using the AtADA2b homolog AtADA2a as bait in this system, a considerably weaker interaction with AtbZIP2, -11 and -44 and hardly any with the group C AtbZIPs could be determined (Figure S3). P2H data were further confirmed making use of co-immunoprecipitation studies, by co-expression of HA-tagged bZIPs and MYC-tagged AtADA2b in Arabidopsis mesophyll protoplasts. Applying an $\alpha$-HA antibody, AtADA2b-MYC protein was efficiently recovered from samples expressing group S1 HA-AtbZIP2, -11 and -44 and HA-AtbZIP10 from group C (Figure 2B). In conclusion, these results support the hypothesis that the transactivation properties of these bZIPs might be ascribed to their ability to recruit the SAGA complex, which is mediated in particular by interaction with the AtADA2b adapter protein. 


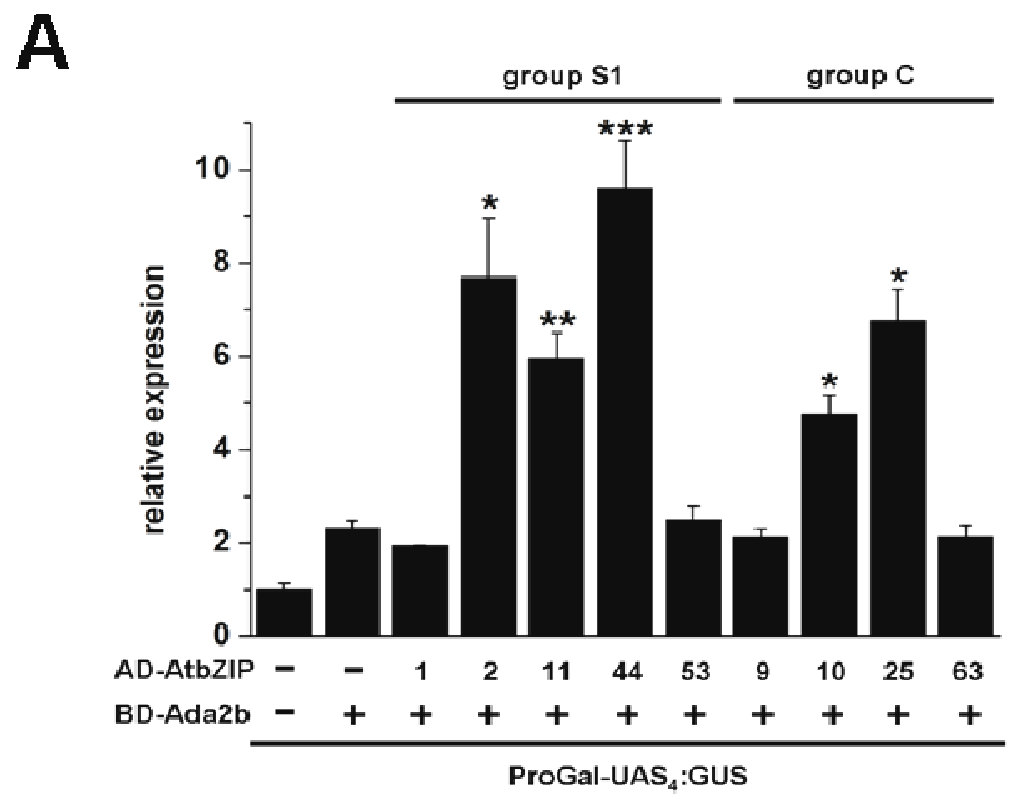

B

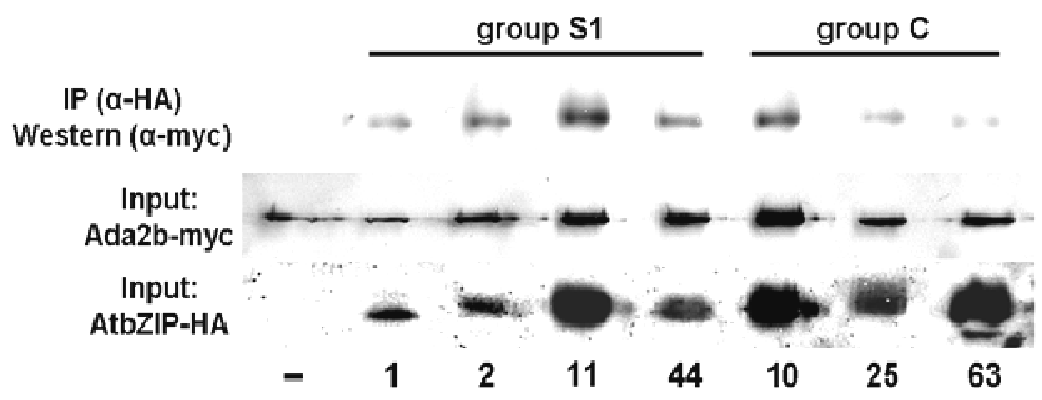

Figure 2: Specific group S1 and C AtbZIP-TFs are able to bind the HAT complex adapter protein AtADA2b in vivo. Binding properties of AtbZIP-TFs were analysed in (A) protoplast two-hybrid (P2H) and (B) co-immunoprecipitation (CoIP) assays. A) In P2H assays, protoplasts were co-transfected with constructs expressing AtADA2b/Gal4-DNA binding domain (BD) and AtbZIP/Gal4-activation domain (AD) fusion proteins, a Gal4-UAS:GUS (ProGal4UAS:GUS) reporter and a Pro35S:NAN normalization construct. Upon interaction of AD and BD fusion proteins, a promoter Gal4 binding TF is reconstituted, that can promote the expression of the reporter. Presented relative expression levels are mean values $( \pm$ SD) from three independent experiments with each of two replicates. They are normalized to the expression of the Pro35S:NAN normalization plasmid and are relative to the background expression of the ProGal4-UAS:GUS reporter. Asterisks mark statistical significant differences between the expression level of each individual assay compared to the expression level, solely driven by the BD-AtADA2b construct $(\mathrm{p} \leq 0.05=* ; \mathrm{p} \leq 0.01=* * ; \mathrm{p} \leq 0.001=* * *)$. B) Mesophyll protoplasts were co-transfected with constructs expressing the HA-tagged AtbZIP proteins indicated and MYC-tagged AtADA2b. Proper protein expression had been determined by immuno-detection (input controls). Complexes of HA-AtbZIP and MYCAtADA2b were isolated using $\alpha$-HA antibody covered magnetic beads and the amount of co-immunoprecipitated MYC-AtADA2b was defined by immuno-detection applying an $\alpha$-MYC antibody (IP samples). Presented is the outcome from one out of three CoIP experiments, which showed similar results. 
The N-terminus of AtbZIP11 and AtbZIP44 functions as an activation domain and is necessary for ADA2b interaction

Based on the fact, that the acidic N-terminus of ZmOpaque-2 was suggested to be crucial for ZmADA2 interaction (Bhat et al., 2004, Barlev et al., 1995), N-terminal truncated AtbZIP11 and AtbZIP44 effector constructs were generated in which the acidic, serine-rich domains (first 26 aa in AtbZIP11 or 40 aa in AtbZIP44, respectively) had been deleted (Figure S4A). To monitor their ADA2 interaction properties, binding of the full-length and N-terminal truncated AtbZIPs to AtADA2b was analysed in $\mathrm{P} 2 \mathrm{H}$ studies. A deletion of the N-terminal domain of AtbZIP11 (Figure 3A) and AtbZIP44 (Figure S4B) strongly compromised their capacity to bind AtADA2b. According to the concept that the expression promoting effect of AtbZIP11 was mediated by recruiting the histone acetylation machinery, we further studied the activation potential of the $\mathrm{N}$ terminal truncated AtbZIP11 and -44 derivatives on the AtGH3.3 promoter in protoplasts. In fact, it became apparent that, although the full-length and truncated proteins were equally expressed, the $\mathrm{N}$-terminal truncated derivatives, in contrast to the full-length AtbZIP11 and -44 failed to promote the AtGH3.3 promoter driven reporter gene expression in the absence of exogenously applied auxin and even suppressed it under auxin-induced conditions (Figure S4C, D).

Similar observations were made in transgenic plants which expressed the bZIP factors in an Estradiol (Est)-dependent manner using the XVE expression system (Zuo et al., 2000). Without additional auxin-treatment, Est-induced AtbZIP11 led to significantly higher expression levels of several auxin-responsive marker genes, which had been found to be controlled by acetylation and deacetylation (Weiste et al., submitted; Figure 3B). In contrast, a comparable expression of the N-terminal truncated AtbZIP $11 \Delta \mathrm{N}$ showed, in particular for AtAUX/IAA3 and AtPIN4 a significantly less induced target gene expression. Whereas ectopic AtbZIP11 expression triggered diverse auxin related phenotypes such as reduction in primary root growth, enhanced lateral root formation and agravitropic root growth, these phenotypes were considerably less pronounced or even not distinct in Est-induced AtbZIP11 $\Delta \mathrm{N}$ plants (Figure 3C), which showed a comparable TF expression (Figure 3D). All in all, these data suggest, that the polar, acidic N-terminus of AtbZIP11 related TFs acts as an activation domain by recruiting the AtADA2b adapter protein of the histone acetylation machinery. 


\section{A}

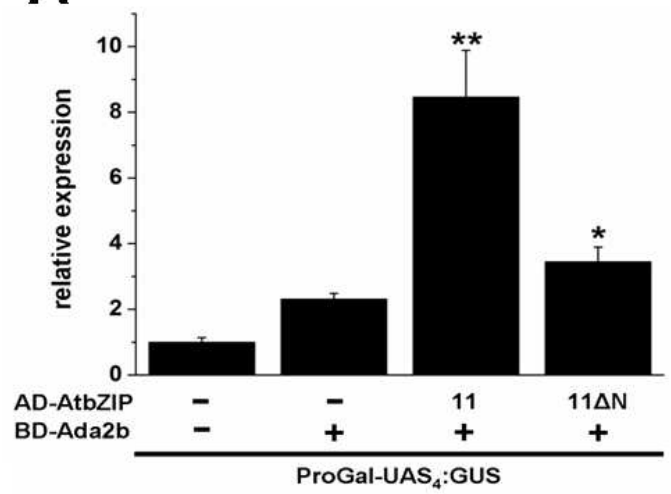

B

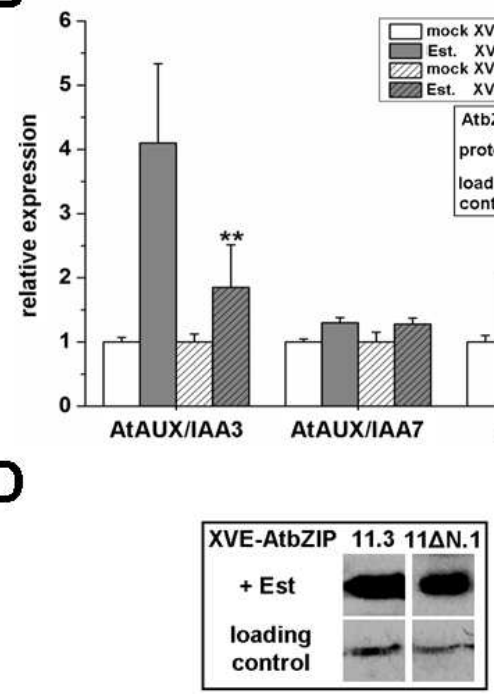

C
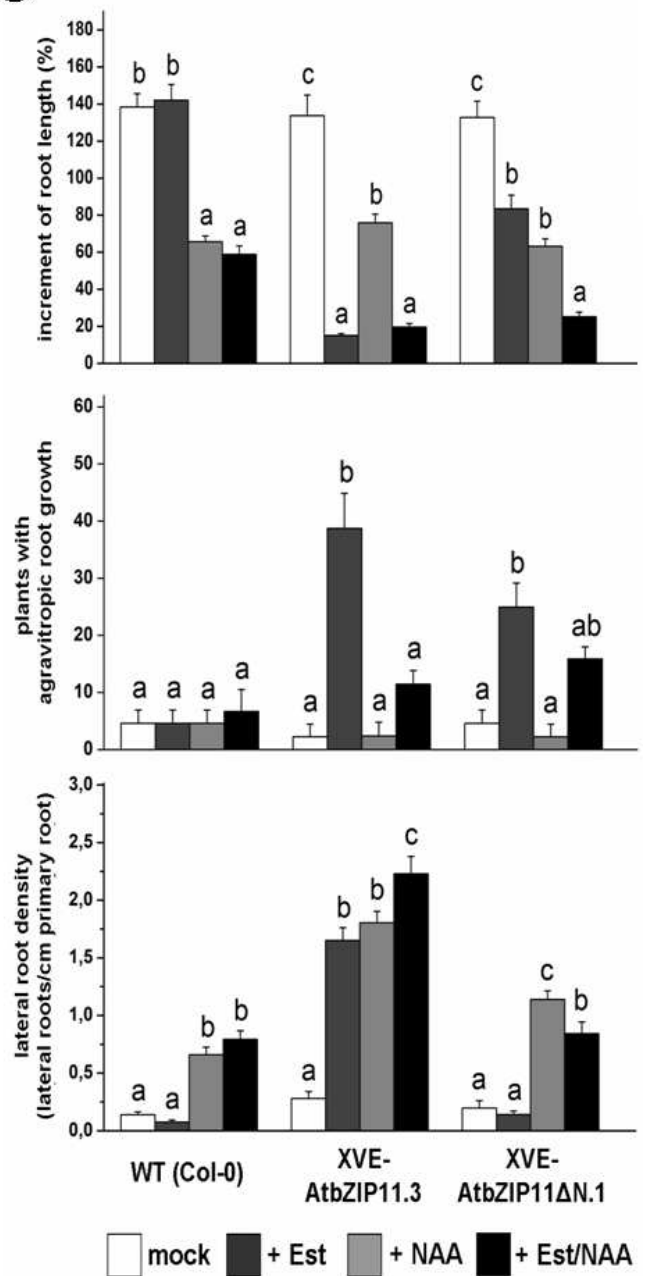

Figure 3: The acidic N-terminus of AtbZIP11 is an activation domain which is crucial for AtADA2b binding. A) The AtADA2b binding capacity of the full-length and N-terminal truncated AtbZIP11-TF protein were analysed in P2H assays. Although the fulllength and N-terminally truncated HA-tagged proteins were equally expressed and stable in protoplasts (see Supplemental Figure S4C), the N-terminal truncated derivative shows, compared to the full-length protein, an impaired capacity to bind AtADA2b. The presented results were obtained from 3 independent transfections with each of 2 technical replicates and are given as mean relative expression values $( \pm \mathrm{SD}$ ). Expression was normalized to the Pro35S:NAN normalization construct and are relative to the expression of the ProGal4-UAS:GUS reporter. B) Transactivation properties of full-length and N-terminally truncated AtbZIP11-TF proteins were studied in transgenic XVE-plants. After 24 hours of induced full-length AtbZIP11-TF expression, a considerably higher target gene expression, with respect to AtAUX/IAA3 and AtPIN4 is observed compared to that induced by similar amounts of AtbZIP11 $\triangle$ N. C) Induced XVE-AtbZIP11 $\triangle \mathrm{N}$ plants exhibit much less severe auxin related root growth phenotypes compared to XVE-AtbZIP11 plants. Typical auxin-related root growth phenotypes such as primary root growth, gravitopic root growth and formation of lateral roots had been studied in WT, transgenic XVE-AtbZIP11 as well as XVE-AtbZIP11 $\Delta \mathrm{N}$ plants. Therefore, 2 weeks old MS grown plants were transferred to, and cultivated for one additional week on, MS plates supplemented with or without $0.25 \mu \mathrm{M}$ NAA and/or $10 \mu \mathrm{M}$ estradiol. For each treatment the root parameters of 44 individual plants were quantified and are given as mean values $( \pm$ SEM). D) Equal expression of AtbZIP11 and AtbZIP11 $\triangle \mathrm{N}$ in transgenic XVE plants had been comfirmed by immuno-detection using a $\alpha$-HA antibody. In the experiments listed above significant differences compared to (A) the expression of the BD-ADA2b or $(\mathbf{B})$ the expression of auxin responsive genes in induced AtbZIP11 and AtbZIP11 $\Delta \mathrm{N}$ plants were determined by Students T-Test and are designated with asterisks ( $\mathrm{p} \leq 0.05=* ; \mathrm{p} \leq 0.01=* * ; \mathrm{p} \leq 0 ., 001=* * *)$. Significant differences between (C) different treatments were defined by one-way ANOVA and subsequent Fisher posthoc test $(\mathrm{p} \leq 0.05)$ and are visualized by different letters. 


\section{Disruption of complex formation between AtbZIP11 related TFs and the} Arabidopsis SAGA complex interferes with auxin-mediated transcription

In order to confirm the pharmacological based results, which showed that expression of auxin- regulated genes is dependent on proper HAT activity, a reverse genetic approach was pursued. As previously demonstrated, expression of an amiRNA construct which significantly reduces the transcript quantity of the highly related AtbZIP2, -11 and -44 TFs genes attenuated the expression of their auxin-responsive target genes (Weiste et al., submitted), which is in line with the hypothesis that these AtbZIPs might be the DNA-binding factors, that specifically recruit the positively acting SAGA complex to the corresponding promoters. Furthermore, Arabidopsis atada $2 a$, atada $2 b$ and atgcn 5 mutants were used to disrupt the histone acetylation machinery. Homozygous mutant plants of HAG1/GCN5 and AtADA2b were found to be strongly dwarfed and display an abnormal root and stamen development, however the phenotypes of atgcn5 mutants are less severe compared to atada2b plants (Vlachonasios et al., 2003). As had been expected this auxin-related phenotypes correlate with similar alterations in the expression of a set of AtbZIP11 inducible, auxin responsive genes in atgcn5 and atada $2 b$ plants. QRT-PCR analyses revealed, that both mutants concurrently showed a substantial reduction in transcript abundance of AtAUX/IAA3/-7 and enhanced expression of $A t G H 3.3$ (Figure 4A), indicating that HAG1/GCN5 is one of the major HATs controlling AtAUX/IAA3/7 expression and at least not the only one regulating $A t G H 3.3$. In fact, AtGH3.3 expression is apparently dependent on the highly homologous (88 \% sequence identity) HAG4 and HAG5 HATs (Latrasse et al., 2008; Cecchetti et al., 2008). In this respect qRT-PCR results from homozygous hag5 mutant plants exhibit a 50\% reduction of AtGH3.3 transcription, whereas AtAUX/IAA3 and -7 expression was largely unaffected (Figure 4B, S5A). This effect on AtGH3.3 expression was even more pronounced in transgenic plants expressing an amiRNA construct targeting both HAG4 and HAG5 transcripts (Figure 4C). Finally, the expression of the auxin responsive marker genes was analysed in homozygous atada2a mutants. In accordance with the hypothesis that AtADA2a and AtADA2b are thought to have distinct biological functions (Hark et al., 2009), expression of AtGH3.3, AtAUX/IAA3 and -7 was, in contrast to their expression in atada $2 b$, not significantly changed in atada2a mutant plants (Figure 4D, S5B). 
In conclusion, it can be stated that the expression of a set of AtbZIP11 inducible, auxinresponsive genes is controlled by specific adapter and HAT components of the histone acetylation machinery.

A

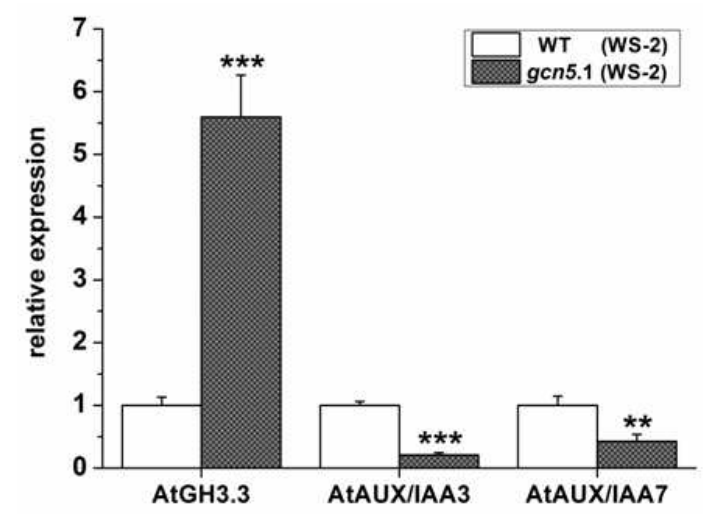

C

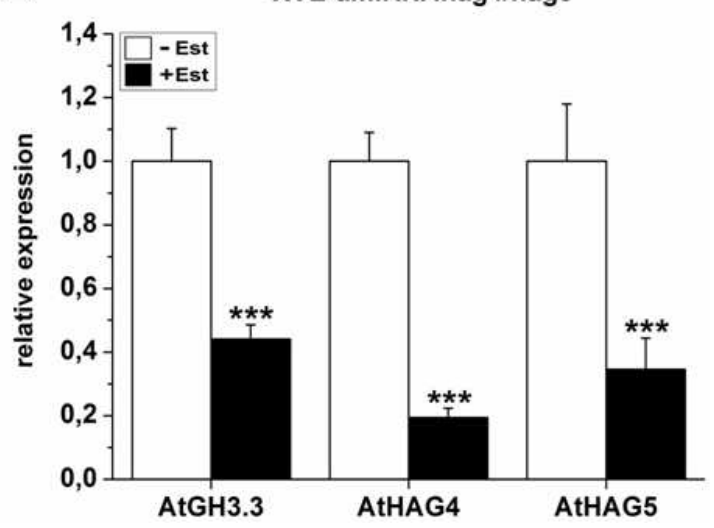

B

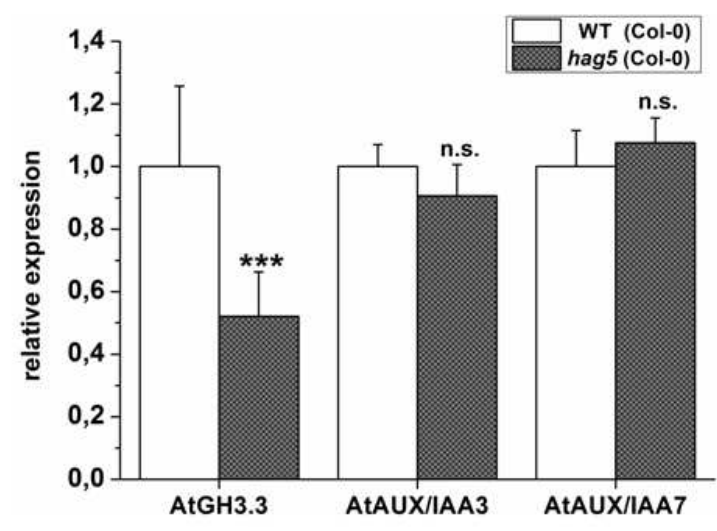

D

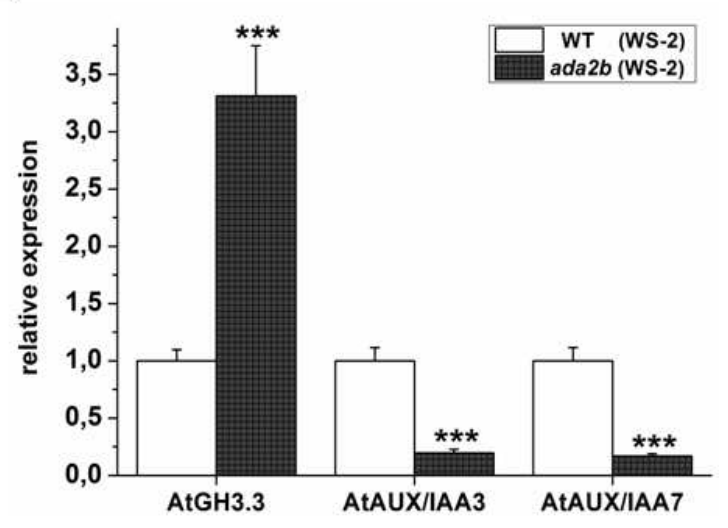

Figure 4: Reverse genetic approaches reveal that auxin responsive genes are regulated by specific Arabidopsis HAT and ADA2 proteins of the histone acetylation complex. Expression of the auxin responsive AtGH3.3, AtAUX/IAA3 and AtAUX/IAA7 genes was quantified by qRT-PCR in the HAT mutants (A) gcn5-1, (B) hag5 or (C) the transgenic XVE-amiRNA-hag4/hag5 knockdown plants. Additionally the expression of these genes was monitored in the ADA2 adapter protein mutant (D) $a d a 2 b-1$. Given are mean relative expression levels ( \pm SEM) from at least 3 individual plants with each of 4 technical replicates. Expression levels are relative to the corresponding WT or uniduced XVE-lines. Significant differences between WT and mutants or uninduced and induced XVE plants are defined by Students T-Test and are marked with asterisks ( $\leq 0.05=* ; \mathrm{p} \leq 0.01=* * ; \mathrm{p} \leq 0.001=* * *)$. 
ChIP analyses reveal that binding of AtbZIP11 related TFs coincides with enhanced GCN5-specific histone acetylation and RNPII recruitment to the auxinresponsive $A t G H 3.3$ promoter

Transcriptional active chromatin is associated with acetylation of specific lysine residues at the N-terminal tail of histone 3, namely K9, K14 and K27. This is thought to enhance the accessibility of the DNA for trans-acting co-activators, thereby facilitating transcription (reviewed in Lee and Workman, 2007; Shahbazian and Grunstein, 2007). As AtbZIP11 and -44 recruit the SAGA complex in a sequence-specific manner, we posed the question if enhanced bZIP binding to the AtGH3.3 promoter correlates with its enhanced histone acetylation and RNPII recruitment. To address this point, CHIP analyses of uninduced and induced XVE-AtbZIP11 and -44 plants were performed applying specific, high-affinity CHIP grade antibodies raised against HA-tagged bZIP fusion proteins, acetylated lysine 27 residues of histone 3, specifically performed by GCN5 (Earley et al., 2007; Benhamed et al., 2006) and the activated RNPII, which is phosphorylated at serin 5 in the C-terminal domain (CTD) of its largest subunit (RPB1) (Mosley et al., 2009). As AtbZIP11 related TFs preferably bind to GREs (Weiste et al., submitted) and envisaged AtbZIP mediated histone acetylation and RNPII binding should also occur in this region, we quantified for each individual CHIP assay the immunoprecipitated GRE rich AtGH3.3 promoter region by qRT-PCR. By this means, we could demonstrate that a short Est-induced expression of either HA-AtbZIP11 or 44 , in the corresponding transgenic XVE-plants, results in an enhanced binding of the AtbZIP-TF, a strongly elevated GCN5 specific H3K27 acetylation and a significantly increased assembly rate of the activated RNPII complex on the AtGH3.3 promoter (Figure 5A, B). In independent experiments it could be further confirmed that at this early induction time-point of AtbZIP11 or AtbZIP44 expression, transcription of the AtGH3.3 gene is already significantly induced (Figure 5C). These in vivo data clearly propose that AtbZIP11 and -44 activate transcription of this auxin responsive gene by chromatin remodelling. 
A

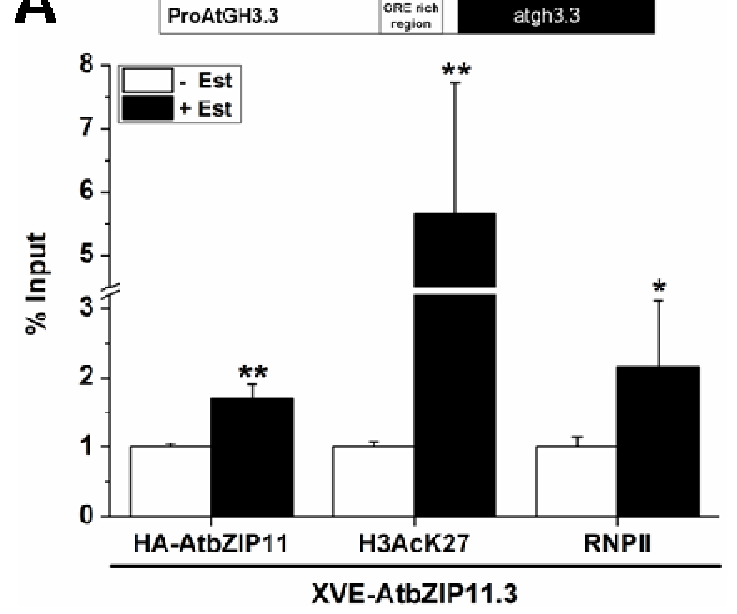

C

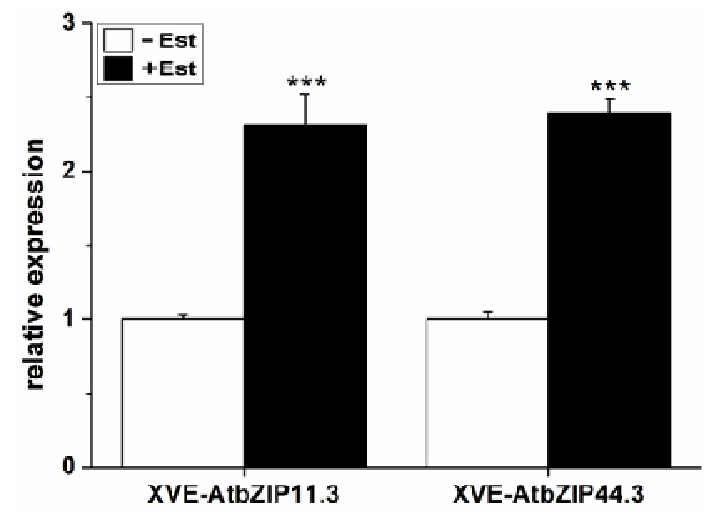

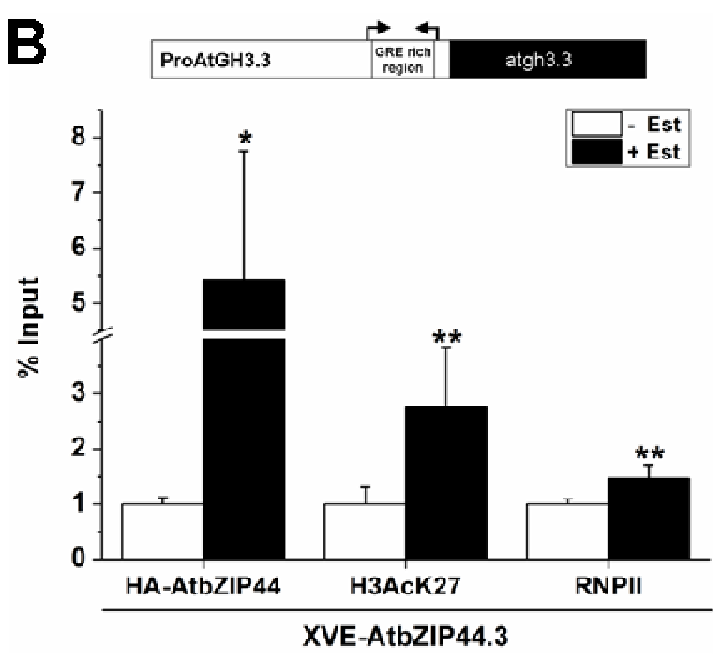

Figure 5: Enhanced binding of AtbZIP11 related TFs to the auxin responsive AtGH3.3 promoter correlates with its enhanced GCN5 specific histone acetylation and RNP II recruitment. Using high-affinity CHIP grade antibodies raised against the HA-tag, GCN5 specific histone 3 lysine 27 acetylation (H3AcK27) and the active RNA Polymerase II (serine 5 phosphorylated RPB1 subunit), ChIP assays were performed to determine if enhanced binding of HAtagged (A) AtbZIP11 or (B) AtbZIP44, coincides with elevated H3AcK27 levels and a higher assembly rate of the activated RNA Polymerase II (RNPII) on the GRE-rich AtGH3.3 promoter region. The amount of immunoprecipitated AtGH3.3 promoter region for each antibody used, was quantified by qRT-PCR using sitespecific primers. Given is the mean relative abundance of the $A t G H 3.3$ promoter region $( \pm \mathrm{SD})$ in shortly induced (6 hrs, $10 \mu \mathrm{M}$ Est.) (black bars) XVE-AtbZIP11 or XVE-AtbZIP44 plants compared to uninduced plants (white bars). Values were normalized to the amount of chromatin DNA used in each ChIP experiment (\% Input), which was specified by qRT-PCR using Actin8 primers. Depicted results were obtained from 2 independent plant pools per line and treatment, from which in total 4 independent ChIP experiments for each antibody were performed. C) Expression of AtGH3.3 was analysed after $6 \mathrm{hrs}$ of induced AtbZIP expression in the corresponding transgenic XVE-plants. Significant differences between the uninduced and induced samples for each ChIP assay (A, B) and from AtGH3.3 expression analysis $(\mathbf{C})$ are defined by Students T-Test and are designated by asterisks $(\mathrm{p} \leq 0.05=* ; \mathrm{p} \leq 0.01=* * ; \mathrm{p}$ $\leq 0.001=* * *)$. 


\section{Discussion}

Under auxin-deficient conditions, the expression of auxin-responsive genes is envisaged to be strictly repressed by AUX/IAA/TPL mediated recruitment of HDACs, leading to histone deacetylation and in consequence to transcriptional inactive chromatin (Szemenyei et al., 2008). As summarized in the model in Figure 6, this study provides conclusive evidence for a complementary bZIP-mediated recruitment of histone acetyltransferases, which specifically open-up the tightly-packed chromatin and thereby, activate auxin-inducible genes.

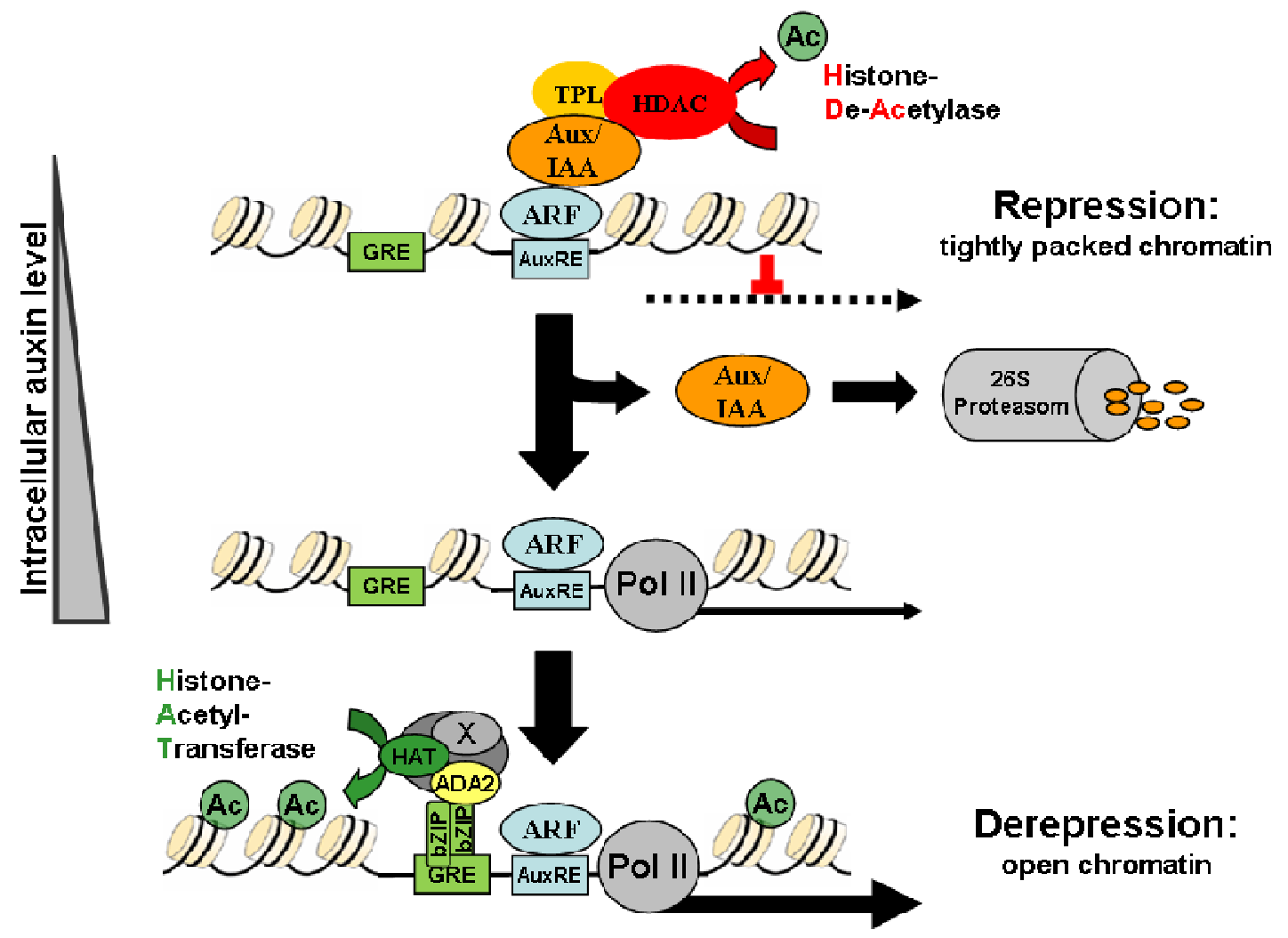

Figure 6: Model of proposed AtbZIP-TF action on auxin mediated transcription. Under auxin deficient conditions, expression of auxin responsive genes is repressed by AUX/IAA proteins which are thought to recruit, via the co-repressor TOPLESS (TPL), histone deacetylases (HDACs) to their target promoters. As soon as auxin concentrations increase, AUX/IAA repressor proteins are marked for degradation by the $26 \mathrm{~S}$ proteasome, via a $\mathrm{SCF}^{\mathrm{TIR}}$ complex dependent mechanism. AUX/IAA degradation de-represses ARF$\mathrm{TF}$ activity which is thus able to induce the expression of ARF-TF target genes. In particular, group S1 AtbZIP-TFs are able to enhance auxin-mediated transcription by ADA2 protein mediated recruitment of histone acetyltransferase complexes. Histone acetylation decondenses the chromatin, thereby facilitating co-factor binding and thus transcription. 


\section{Chromatin remodelling by histone acetylation and deactylation is a crucial mechanism for the regulation of auxin-responsive genes}

Making use of pharmacological approaches, chromatin remodelling by histone acetylation and deacetylation has been defined to be a crucial mechanism in auxinregulated transcription. Inhibition of HDACs by TSA de-represses transcription of several auxin-induced genes, although the impact differs considerably depending on the gene under investigation. These results suggest that this mechanism is generally conserved. Nevertheless, it is further fine-tuned to establish typical gene-specific expression patterns. In contrast, inhibition of acetylation by a GCN5 specific HAT inhibitor, represses auxin incuced transcription of the analysed auxin responsive genes, which is in line with the model, that their auxin mediated expression is facilitated by a histone deacetylation counteracting system. The results from the pharmacological studies were complemented by reverse genetic approaches, demonstrating that AtGCN5 and AtADA2b, both protein components of the Arabidopsis SAGA-complex are crucial for auxin-induced gene activation.

Consistent with this, recently published results highlight the relevance of the AtADA2b adapter protein in auxin-induced gene regulation (Anzola et al., 2010) and suggest a relevance of the HAT GCN5 in an AUX/IAA/TPL counteracting mechanism (Long et al., 2006). Besides the role of GCN5, we have demonstrated that further HATs of the GNAT/MYST superfamily are involved in controlling auxin-mediated transcription. However, it has to be pointed out, that a certain specificity of the studied HATs (HAG1/GCN5, HAG4 and HAG5) and adapter proteins (ADA2a, ADA2b) with respect to auxin-responsive gene regulation is observed.

\section{BZIP transcription factors are mediators which specificly recruit the histone} acetlyation machinery to GRE motifs in auxin-inducible promoters

In order to recruit the SAGA acetylation machinery to a promoter in a sequencespecific-manner, DNA-binding TFs are required. Recent studies have implicated AtbZIP11, -2 and -44 as quantitative modulators of auxin-responsive gene expression which specifically bind GRE cis-elements (Weiste et al., submitted). Several biased and unbiased bioinformatic approaches have revealed that GREs, which are preferred binding sites for plant bZIP-TFs (Jakoby et al., 2002) are significantly enriched in auxin 
responsive promoters from the dicot model plant Arabidopsis and the monocot plant rice (Nemhauser et al., 2004; Pufky et al., 2003; Berendzen and Weiste et al., in preparation). Furthermore, an observed enrichment of composite GRE-AuxRE ciselement modules in promoters of auxin inducible genes from both species indicated that GREs are conserved, quantitative coupling motifs of the well-described auxinresponsive element (AuxRE) (Berendzen and Weiste et al., in preparation). This assumption had been further underpinned by mutational promoter cis-element analysis using the early auxin-responsive soybean GH3 (Ulmasov et al., 1995) or Arabidopsis AtGH3.3 promoter (Weiste et al., submitted).

To elucidate the mechanism by which the GRE/AtbZIP module is enhancing AuxRE mediated transcription, an envisaged direct protein-protein interaction between AtbZIP and ARFs was examined, but couldn't be confirmed, yet (CW, WDL unpublished results). However, we found that AtbZIP11 and -44, which specifically bind GREs, directly interact with AtADA2b, which is an important component of the SAGA complex, using in vivo $\mathrm{P} 2 \mathrm{H}$ and Co-IP studies. In line with this, approaches which interfere with endogenous AtbZIP expression reveal an impairment of auxin-induced transcription. As T-DNA insertion lines for the individual highly homologous group S1 AtbZIP2, -11 and -44 appear to be lethal, a multi-target Est-inducible amiRNA approach was established. Induced expression of this amiRNA results both in significantly reduced expression of AtbZIP11/2/44 as well as of its putative auxininduced targets (Weiste et al., submitted).

This data supports the view, that group S1, AtbZIP11 related TFs function as promoterspecific adapters, recruiting the acetylation machinery. Comparing several bZIP factors in interaction assays, we could demonstrate that the ADA2 recruitment is not specific to AtbZIP11 or -44 but is conserved within several but not all group C and S1 bZIP factors. As it has been demonstrated for O2 is maize (Bhat et al., 2004; Locatelli et al., 2009), the closely homologous Arabidopsis group C members AtbZIP10 and -25 also interact with AtADA2 proteins. Interestingly, O2 and AtbZIP10, -25 have been shown to exert related functions in regulation of seed storage protein genes in maize and Arabidopsis (Locatelli et al., 2009; Alonso et al., 2009). Hence these data suggest that bZIPs are not only involved in recruiting the acetylation machinery during auxininduced transcription. Indeed, recent publications implicate seed specific gene 
regulation with histone acetylation (Locatelli et al., 2009; Bhat et al., 2003). As GREs frequently act as quantitative cis-elements in a wide array of stimulus-induced gene expressions (Dietrich et al., 2011; Baena-Gonzalez et al., 2007; Alonso et al., 2009), it is tempting to speculate that GRE-mediated recruitment of SAGA-complexes is of more general importance. However, further functional studies are needed to define the function of bZIPs on a genome-wide level.

As the interaction between $\mathrm{O} 2$ and ZmADA2 is thought to be mediated by its acidic, $\mathrm{N}$ terminal activation domain (Bhat et al., 2004; Barlev et al., 1995), we accordingly tested if the acidic N-terminus of AtbZIP11-related TFs is also necessary for activating auxinresponsive transcription and AtADA2b binding. In fact, $\mathrm{N}$-terminal truncated derivatives of AtbZIP11 and -44 exhibit a significant reduced capacity to bind AtADA2b and to enhance the expression of their auxin-responsive target genes. In agreement with this, also the AtbZIP11 induced, auxin-related root phenotypes were found to be much less pronounced in plants expressing the truncated TF protein. These data indicate that the interaction platform is not strictly conserved but probably is characterized by specific features, such as polar, acidic amino acid residues which are frequently found in activation domains (Schmitz et al., 1997). Interestingly, the recruitment properties of the bZIPs for specific adapter proteins differ. For example AtbZIP11, -2 and -44 recruit both AtADA2b and AtADA2a, although with significant lower affinity for the latter. In contrast, adapter binding of group C, AtbZIP10 and -25 appear to be only specific to AtADA2b. Accordingly, expression of auxin-induced genes is not affected in atada2a mutants but strongly impaired in atada2b. Along this line, auxin related phenotypic alterations are only pronounced in atada $2 b$ mutants, which cannot be rescued by AtADA2a expression (Hark et al., 2009; Vlachonasios et al., 2003). This indicates that the adapter proteins likely implement specific functions by recruiting at least partially individual sets of transcriptional regulators.

Comparing auxin-induced expression of AtGH3.3, AtAUX/IAA3 and AtAUX/IAA7 in mutant lines of AtADA2b and AtGCN5 the same pattern can be observed, supporting the model that both proteins functionally co-operate in the SAGA complex. Unexpectedly, AtGH3.3 expression is strongly enhanced in the hagl (atgcn5) and atada $2 b$ mutants, whereas expression of the $A U X / I A A 3$ and -7 genes is significantly reduced. As these repressor proteins had been demonstrated to negatively regulate 
AtGH3.3 expression (Weiste et al., submitted), likely by histone deacetylation, the expression of AtGH3.3 is de-repressed in hagl and ada2b mutant plants and no longer dependent on the recruitment of the counteracting histone acetylation machinery.

Due to this fact, the significance of GCN5 for AtGH3.3 expression is hard to address. Nevertheless, the hag4 mutant and the multi-target amiRNA line, suppressing both HAG4 and HAG5 expression reveal that the expression of the AtGH3.3 gene is at least dependent on these HATs, whereas AUX/IAA3 and -7 expression is not.

In line with published work (Locatelli et al., 2009; Mao et al., 2006) it is tempting to speculate that various HAG and ADA proteins interact to form distinct SAGAcomplexes which appear to be recruited to distinct sets of target genes. However, neither the mechanism, nor the impact on gene regulation is understood, so far. Altogether, these data support an important mediator function of AtbZIP11 and -44 in recruiting specific SAGA complexes, via their acidic activation domains.

\section{By binding to auxin-induced promoters AtbZIP11 and AtbZIP44 stimulate chromatin remodelling and enhance RNA Polymerase II dependent transcription}

The mechanistic model based on pharmacological, biochemical and genetic data was further supported by in vivo experiments. ChIP analyses confirm that Est-induced bZIP expression leads to an enhanced binding of the AtbZIP11 and -44 TFs to the AtGH3.3 promoter sequence which harbours several GRE binding site. This binding coincides with enhanced GCN5 specific histone 3, lysine 27 acetylation and recruitment of RNPII which consequently results in enhanced transcription of AtGH3.3. Recently we proposed the GRE/bZIP module to act as a "rheostat" to fine-tune AuxRE-mediated auxin-responses (Weiste et al., submitted). In here, we provide a mechanism, how this module is controlling transcription via SAGA-recruitment and chromatin remodelling. As the identified bZIPs belong to the C/S1 network of AtbZIP-TFs, which are known to specifically form heterodimers and to be involved in reprogramming the metabolism in response to low energy stress (Hanson et al., 2008; Dietrich et al., 2011) it is tempting to speculate, that the described regulatory mechanism is used to adjust auxin-mediated growth responses according to the energy status of the cell. 


\section{Material \& Methods}

\section{Plant material, transformation and culture}

Arabidopsis thaliana ecotype Columbia (Col-0) or Wassilewskija (WS-2) and hag5 (At5g09740; SALK_106046C), ada2a-3 (Hark et al., 2009), ada2b-1 and gcn5-1 (Vlachonasios et al., 2003) mutant plants were cultivated on soil under long day conditions (16 h light $/ 8 \mathrm{~h}$ dark) at $23{ }^{\circ} \mathrm{C}$ and a relative humidity of $60 \%$. The genotype of all mutant plants and the expression of mutant alleles from yet uncharacterized mutants was confirmed by PCR/ qRT-PCR using gene-, T-DNA- or transcript specific primers, respectively (see Table S1).

Transgenic lines were generated using the "Floral Dip Transformation" technique applying the Agrobacterium tumefaciens strain GV3101 (Clough and Bent, 1989). For expression analyses and root morphology assays of transgenic XVE-lines surface sterilized and stratificated seeds were cultivated on 1/4 MS (Murashige and Skoog, 1962) agar plates without sugars under long day conditions. Prior to expression analysis by qRT-PCR two weeks old Est inducible plants were treated with $7 \mu \mathrm{M}$ Est (17-ß3estradiol-E2758, Sigma-Aldrich Chemie GmbH, Munich, Germany) in PBS or an appropriate mock solution for 6 or $24 \mathrm{~h}$, respectively. For expression analysis of XVEamiRNA-bZIP2/11/44 lines, the plants were preinduced with $10 \mu \mathrm{M}$ Est for $20 \mathrm{~h}$ to ensure target mRNA degradation prior to gene induction by an additional auxin treatment $(2 \mu \mathrm{M}$ NAA for $4 \mathrm{~h})$.

Plants analysed in root morphology assays were grown for two weeks on agar plates in vertical position before they were transferred for an additional week on inductive medium supplemented with or without $0.25 \mu \mathrm{M}$ NAA and/or $10 \mu \mathrm{M}$ Est.

\section{Protoplast transformation and culture}

Protoplast transformation has been performed as described by Sheen et al., (2001) with modifications according to Ehlert et al., (2006). For auxin treatment, protoplasts were incubated over night in WI solution supplemented with $0.25 \mu \mathrm{M}$ NAA (Sigma-Aldrich Chemie $\mathrm{GmbH}$, Munich, Germany company). In standard transactivation experiments 9 $\mu \mathrm{g}$ of reporter plasmid, $3 \mu \mathrm{g}$ of a NAN normalization plasmid (Kirby and Kavanagh, 
2002) and $14 \mu \mathrm{g}$ of an effector plasmid has been used. Concerning protoplast twohybrid analyses, $9 \mu \mathrm{g}$ of the $\mathrm{ProGal}_{4} \mathrm{UAS}$ :GUS reporter construct (B. Weisshaar, University of Bielefeld, Germany), $3 \mu \mathrm{g}$ of the NAN normalization plasmid and $14 \mu \mathrm{g}$ of Gal4-binding domain (BD) and/or Gal4-activation domain (AD) effector fusion constructs were applied.

In HAT and HDAC inhibitor studies, protoplasts were cultivated for $4 \mathrm{hrs}$ in isotonic WI buffer supplemented with or without $0.8 \mu \mathrm{M}$ TSA (Cell Signaling Technology, USA), or 0.25 mM y-butyrolacton (ALX-270-411; Axxora GmbH, Lörrach, Germany), prior to an additional $2 \mathrm{hrs}$ incubation period with the inhibitors and $2 \mu \mathrm{M}$ NAA or an adequate mock additive (DMSO).

\section{Molecular biological techniques}

Standard DNA techniques have been described in Sambrook et al., (1989). DNA sequence analyses were performed using an ABI310 sequencer (Applied Biosystems, Darmstadt, Germany) with an ABI PRISM BigDye terminator cycle sequencing reaction kit (Applied Biosystems, Darmstadt, Germany). Vector DNA was gel extracted and prepared using commercial kits (Macherey-Nagel GmbH \& Co. KG, Düren, Germany). Western analysis has been performed making use of a primary polyclonal $\alpha$ HA antibody from rabbit (Santa Cruz, Santa Cruz, CA, USA) or a monoclonal $\alpha-$ MYC antibody from mouse (Cell Signaling Technology, USA) and a secondary anti-rabbit or anti-mouse IgG conjugated with a horseradish peroxidase (GE Healthcare, Freiburg, Germany). QRT-PCR has been performed as described in Dietrich et al., (2011) and ChIP analyses according to Fode et al., (2009) with minor modifications. Instead of sepharose beads, protein A precoated, magnetic dynabeads (Invitrogen $\mathrm{GmbH}$, Darmstadt, Germany) were applied. For each IP, $15 \mu \mathrm{g}$ chromatin and $5 \mu \mathrm{g}$ CHIP grade antibody (Abcam Cambridge, UK); CHIP grade $\alpha$-HA (ab9110), CHIP grade Histone H3 acetyl K27 (ab4729) and CHIP grade RNA polymerase II CTD repeat YSPTSPS (phosphor S5) were used. All qRT-PCR oligonucleotide primers used are summarized in Table S1. 


\section{Co-immunoprecipitation assays}

Protocol for Co-IP assays was adapted from Shan et al., (2008) with minor modifications. $2 \mathrm{ml}$ protoplasts $(2 \times 105 / \mathrm{ml})$ were transfected with $200 \mu \mathrm{g}$ of Pro35S:HA-AtbZIP and/or Pro35S:MYC-AtADA2b effector constructs. Proteins from transfected protoplasts were extracted in a cooling chamber at $4^{\circ} \mathrm{C}$ adding $0,5 \mathrm{ml}$ extraction buffer (10 mM HEPES [pH 7.5], $100 \mathrm{mM} \mathrm{NaCl}, 1 \mathrm{mM}$ EDTA, $10 \%$ glycerol, $0.5 \%$ Triton X-100, and a protease inhibitor cocktail from Roche). After lysing the cells by vigorously vortexting for $30 \mathrm{~s}$, cell debris was removed by centrifugation at $13,000 \mathrm{rpm}$ for $15 \mathrm{~min}$ at $4^{\circ} \mathrm{C}$. The supernatant was incubated with 2 $\mu \mathrm{g}$ of a CHIP-grade $\alpha$-HA antibody (ab9110; Abcam Cambridge, UK) for $1 \mathrm{hr}$ at $4^{\circ} \mathrm{C}$ with gentle shaking, prior to an additional incubation for 2 hrs with $70 \mu \mathrm{l}$ of a prewashed, protein A coated, Dynabead/extraction buffer solution. Subsequently beads were collected using an appropriate magnet and washed four times with washing buffer (10 mM HEPES [pH 7.5], 100 mM NaCl, 1 mM EDTA, $10 \%$ glycerol, $0.1 \%$ Triton X100 , and a protease inhibitor cocktail). Bound proteins were eluted with a 5x SDS/urea sample buffer and subjected to SDS-PAGE. Co-immunoprecipitated proteins were analysed by immuno-detection using an $\alpha$-HA or $\alpha$-MYC antibody. The protein bands of expected molecular weights are presented. Shown are the data from one out of three CoIP experiments, which showed comparable results.

\section{Vector construction}

The ProAtGH3.3:GUS reporter construct used in the transient protoplast transactivation assays was created by amplifying the promoter sequence including the 5 UTR from AtGH3.3 ( $1700 \mathrm{bp}$ ) from Arabidopsis WT genomic DNA by PCR using the primers listed in Table S1. Making use of the attached flanking restriction sites of the resulting PCR products (XbaI, NcoI) the promoter fragment was inserted into the reporter plasmid pBT10-GUS (B. Weisshaar, University of Bielefeld, Germany). The AtbZIP group $\mathrm{S} 1$ and $\mathrm{C}$ bZIP effector plasmids (HA, BD and AD fusions) used in the transactivation assays are described in Ehlert et al., (2006). Entry clones for AtADA2a/b were obtained from Steven J. Triezenberg, Michigan State University, USA. N-terminal truncated AtbZIP11 and AtbZIP44 constructs were created by amplifying the corresponding gene fragment from the AtbZIP11 and 44 full-length entry clones using 
primers attaching new gateway recognition sites (Table S1). Using the Gateway technology® (Invitrogen, Darmstadt, Germany), the genes stored in entry clones were transferred into the transient pHBTL- HA/AD/BD expression vectors (Ehlert et al., 2006). For CoIP experiments the transient pHBTL-MYC expression vector was constructed. Therefore the Pro35S:HA-gateway cassette fragment from the pHBTL-HA vector was exchanged by the Pro35S:4xMYC-gateway cassette from the binary pGWB18 vector (Nakagawa et al., 2007) using Afl II and Hind III restriction enzymes. In order to generate stable transformed Arabidopsis plants the Est inducible XVE system was used. The gateway compatible binary pMDC7 vector (Zuo et al., 2000) was applied for inducible expression of amiRNA constructs. Target specific and efficient amiRNA sequences were identified using the online amiRNA design tool WMD2 (http://wmd2.weigelworld.org).

To generate Est inducible over-expressors and to monitor target gene expression a HAtag was incorporated into the pMCD7 vector. The HA-tag was PCR amplified from the pEHA vector (Weiste et al., 2007) attaching XhoI restriction sites and inserted between the inducible promoter and the gateway attachment site of the pMDC7 vector.

\section{Quantification of auxin related root growth phenotypes}

To determine root morphology parameters high resolution images (5 Megapixel, 24 bit) of 44 individual plants per treatment were taken using the Camag reprostar 3 documentation system with a Canon G5 camera (CAMAG AG \& Co. GmbH, Berlin, Germany). From these pictures the root parameters of the differently treated plants were monitored. These are: lateral root number, the abundance of roots with obvious agravitropic root growth and the total root length before and after one week of treatment. The root length was measured using the Image $\mathrm{J} 1.43 \mathrm{u}$ software available at http://rsb.info.nih.gov/ij, whereas the number of lateral roots and roots with agravitropic growth was determined by manual counting.

\section{Statistics and Alignments}

Figures and statistical tests were done applying the OriginPro 8.1G and Statgraphics Centurion XVI software. Significant differences between multiple constructs and 
treatments were determined using the One-way ANOVA test followed by a Fisher posthoc test $(\mathrm{p} \leq 0.05)$ and are visualized by different letters. Significant differences between only two datasets are defined making use of the Students T-Test and are labeled with asterisks $(\mathrm{p} \leq 0.05=* ; \mathrm{p} \leq 0.01=* * ; \mathrm{p} \leq 0.001=* * *)$. Protein sequence alignments were performed with Vector NTI 10 software (Invitrogen GmbH, Darmstadt, Germany).

\section{Acknowledgements}

We are grateful to Anna Hermann and Jennifer Krüger for valuable technical assistance and K. Harter for proof reading. We thank B. Weisshaar (University of Bielefeld, Germany) and N.-H. Chua (Rockefeller University, NY, USA) for plasmids and C. Gatz, V. Lipka and J. Schirawski (University of Göttingen, Germany) for sharing lab facilities. This research was supported by grants from the Deutsche Forschungsgemeinschaft (DFG DR273-10/2).

\section{Supplemental figures}

Supplemental Table 1: List of applied oligonucleotide primers

Supplemental Figure 1: Protein sequence alignment of Arabidopsis homologs of the yeast and human histone acetyltransferase GCN5

Supplemental Figure 2: Expression of the Ubiquitin5 gene is unaffected by auxin and the applied HAT and HDAC inhibitors

Supplemental Figure 3: AtADA2a binding properties of group C and S1 AtbZIP-TFs Supplemental Figure 4: The acidic, polar N-terminus of AtbZIP11 related TFs is an activation domain and is crucial for AtADA2b binding

Supplemental Figure 5: Expression analysis of AtHAG5 in homozygous hag5 and of auxin responsive genes in ada2a mutant plants. 
Supplemental Table 1: List of applied oligonucleotide primers. Primers used in this work for promoter and gene cloning, qRT-PCR, CHIP analysis and plant genotyping are presented. Primer sequences are given in 5' to 3' orientation.

\begin{tabular}{|c|c|c|}
\hline category & primer & sequence $\left(5^{`}-3^{\prime}\right)$ \\
\hline \multirow{6}{*}{$\begin{array}{l}\text { 옹 } \\
\text { 응 }\end{array}$} & ProAtGH3.3 for & AAAAATCTAGAGTGCTGAATATTTT \\
\hline & ProAtGH3.3 rev & AAAAACCATGGGATTAAAATGGTAT \\
\hline & AtbZIP11 $\Delta \mathrm{N}$ for & GGGGACAAGTTTGTACAAAAAAGCAGGCTCCAGGAAACGTAAACGGATGCTC \\
\hline & AtbZIP11 $1 \Delta \mathrm{N}$ rev & GGGGACCACTTTGTACAAGAAAGCTGGGTCTTAATACATTAAAGCATCAG \\
\hline & AtbZIP $44 \Delta \mathrm{N}$ for & GGGGACAAGTTTGTACAAAAAAGCAGGCTCCCGGAAGAGAAAGAGGAAACAG \\
\hline & AtbZIP $44 \Delta \mathrm{N}$ rev & GGGGACCACTTTGTACAAGAAAGCTGGGTCCTAACAGTTGAAAACATCACC \\
\hline \multirow{20}{*}{ 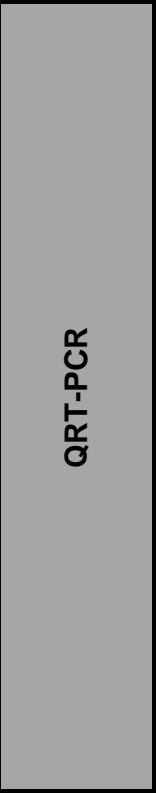 } & AtGH3.3 RT for & CATCACAGAGTTCCTCACAAGC \\
\hline & AtGH3.3 RT rev & GTCGGTCCATGTCTTCATCA \\
\hline & AtAUX/IAA3 RT for & AAAGGCTCAGATTGTTGGATGGC \\
\hline & AtAUX/IAA3 RT rev & TGACCCTCATGCTCAGATTCATTC \\
\hline & AtAUX/IAA7 RT for & AAGCTACCAGGATCTTTCTGATGC \\
\hline & AtAUX/IAA7 RT rev & ATTCCTTGTGCTCCATAGTTTCCC \\
\hline & AtPIN4 RT for & TTGTCTCTGATCAACCTCGAAA \\
\hline & AtPIN4 RT rev & ATCAAGACCGCCGATATCAT \\
\hline & AtbZIP2 RT for & TGATCGGAAACTGATGACTCC \\
\hline & AtbZIP2 RT rev & GAGCAGATTTGACCGTGAGC \\
\hline & AtbZIP11 RT for & CGATTCAAACGTCGTCAGG \\
\hline & AtbZIP11 RT rev & TCCGTTTACGTTTCCTCTGC \\
\hline & AtbZIP44 RT for & CATCTACGTAAAGAAAACGCTCAG \\
\hline & AtbZIP44 RT rev & CCGGTCTCCATACCGAATC \\
\hline & AtUBQ5 RT for & GACGCTTCATCTCGTCC \\
\hline & AtUBQ5 RT rev & GTAAACGTAGGTGAGTCCA \\
\hline & AtHAG4 RT for & TGTTTGAGGTGGATGGCAAGAAG \\
\hline & AtHAG4 RT rev & GGCATCCACGATCATCACATTCAC \\
\hline & AtHAG5 RT for & CAACGGCAACGCACCG \\
\hline & AtHAG5 RT rev & AAAGGAGGAGGAGGAGGATGAGA \\
\hline \multirow{4}{*}{$\frac{\stackrel{\varrho}{\bar{T}}}{\text { U }}$} & CHIP PromAtGH3.3 for & TGCCAACGTGGCGCAGACATATCAGTCCC \\
\hline & CHIP PromAtGH3.3 rev & CAAGAAGGAGAAAACCAACGTCAGCC \\
\hline & CHIP AtActin8 for & GGTTTTCCCCAGTGTTGTTG \\
\hline & CHIP AtActin8 rev & СTCCATGTCATCCCAGTTGC \\
\hline \multirow{11}{*}{ 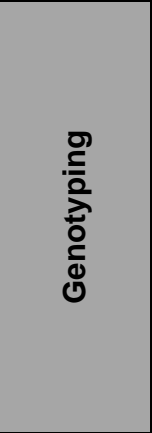 } & ada2a-3 (SALK) geno for & CTAGCTTCTCGTCCTGCTGAGG \\
\hline & ada2a-3 (SALK) geno rev & TTGACTCCAGCTAAGGCAGACAAAG \\
\hline & ada2b-1 (UW) geno for & АСTCCTCACAAATGTGATCACCCATACCG \\
\hline & ada2b-1 (UW) geno rev & GGTGGAAACAGGTTTCTTCCCTCCAAAAC \\
\hline & gcn5-1 (UW) geno for & AGTGGGGGCACACTCGTTTCAAATTATTC \\
\hline & gcn5-1 (UW) geno rev & TCCGCAACAAACATATCCAATGTCACGTA \\
\hline & hag5 (SALK) geno for & GCTGAGTAATATGATGACAAAGTTGGTG \\
\hline & hag5 (SALK) geno rev & AATGCAACTTTAATGAACCGTGAATG \\
\hline & LBa1 (SALK T-DNA left border) & TGGTTCACGTAGTGGGCCATCG \\
\hline & JL202 (UW T-DNA left border) & CATTTTATAATAACGCTGCGGACATCTAC \\
\hline & XR-2 (UW T-DNA right border) & TGGGAAAACCTGGCGTTACCCAACTTAAT \\
\hline
\end{tabular}




\begin{tabular}{|c|c|c|}
\hline & & 80 \\
\hline Yeast GCN5 & (1) & $---\mathrm{MVTKHQI}$ \\
\hline Human GCN5 & (1) & -----MLEEE IYGANSPI \\
\hline AtHAG1 (GCN5) & (1) & MDSHSSHLNAANRSRSSQTPSPSHSASASVTSSLHKRKLAATTAANAAASEDHAPPSSSFPPSS FSADTRDGALTSNDEL \\
\hline AtHAG4 (HAM1) & (1) & ---MGSSADTETAMI IATPASNHNNPATNGGDANQNHT SG---------------AILALTNSESD \\
\hline AtHAG5 (HAM2) & (1) & ------- MGSSANTETNGNAPPPS SNQKPPATNGVDGSHPPP PP---n----------LTPDQAIIESD \\
\hline Consensus & (1) & AT $\bar{G} \quad \bar{A}$ \\
\hline & & 160 \\
\hline Yeast GCN5 & (8) & EEDHLDGATTDPEV---KRVKLENNVEEIQPEQAET---------NKQ-----EGTDKENKGKFEKETERIGGSEVVTDV \\
\hline Human GCN5 & (14) & WESGFTMPPSEGTQLVPRPASVSAAVVPSTPIFS PSMGGGSNSSLSLDSAGAEPMPGEKRTLPENLTLEDAKRLRVMGDI \\
\hline AtHAG1 (GCN5) & (81) & ESISARGADTDSDPDESEDIVVDDDEDEFAPEQDQDSSIRTFTAARLDSSSGVNGSSRNTKLKTESSTVKLESSDGGKDG \\
\hline AtHAG4 (HAM1) & (49) & ASKKRRMGVLPLEVGTRVMCQWRDGKYHPVKVIERR-KNYNGGHNDYEYYVHYTEFNRRLDEWIKLEQLDLDSVECALDE \\
\hline THAG5 (HAM2) & (49) & PSKKRKMGMLP LEVGTRVMCRWRDGKHHPVKVIERR-RIHNGGQNDYEYYVHYTEFNRRLDEWTQLDQLDLDSVECAVDE \\
\hline Consensus & (81) & $\begin{array}{crlrlrr}S & \text { RMA TD EV } & \text { V DG } & \text { PV E } & \text { D } & \text { RR } & \text { LE LDLDSVE A D } \\
161 & & & & & & \mathbf{2 4 0}\end{array}$ \\
\hline Yeast GCN5 & (71) & 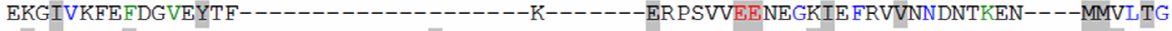 \\
\hline Human GCN5 & (94) & PMELVNEVMLT ITDPA-----------A-MLGPETSLLSANAARDETARLEE RRGI IE FHVIGNSLTPKANRRVLLWLVG \\
\hline AtHAG1 (GCN5) & (161) & GSSVVGTGVSGTVGGSS ISGLVPKDESVKVLAENFQTSGAYIAREEALKREE QAGRLKFVCYSNDSIDEH----MMCLIG \\
\hline AtHAG4 (HAM1) & 28) & KVEDKVTSLKMTRHQ-------KRKIDETHVEGHEELD------AAS LREHEE FTKVKN IATIELGKYEIE----TWYFSP \\
\hline AtHAG5 (HAM2) & 28) & KVEDKVTSLKMTRHQ------KRKIDETHIEGHEELD------AASLREHEE FTKVKN ISTIELGKYEIE----TWYFSP \\
\hline Consensus & $(161)$ & 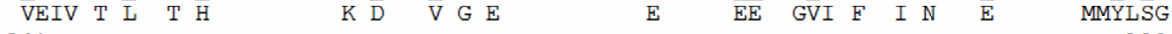 \\
\hline & & 241 \\
\hline Yeast GCN5 & 20) & KMPKEYIARLVYDRSHLSMAVIRKPLTVVGGITYRPFDKREFA \\
\hline Human GCN5 & 62) & LQNVESHQLPRMPKEYIARLVFDPKHKTLALIKDGR-VIGGICFRMFPTQGET IVA CAVTSNEQVKGYGTHLMNHLKEY \\
\hline AtHAG1 (GCN5) & (37) & LKNIFARQLPNMPKEYIVRLIMDRKHKSVMVLRGNL-VVGGITYRPYHSQKF \& E AEAAITADEQVKGYGTRLMNHLKQH \\
\hline tHAG4 ( HAM1) & 92) & FPPEYNDCVKL FFCEFCLSFMKRKEQ---------LQRHMRKCDLKHPPG A I YASSTLSMFEVDGK--------- \\
\hline LHAG5 (HAM2) & 92) & FPPEYNDCVKLFFCEFCLNFMKRKEQ----------LQRHMRKCDLKHPPGD \\
\hline Consensus & (241) & $\begin{array}{rrr}\text { VVGGI YR F F EF ECAITS EQVKGYG LMNHLK Y } \\
400\end{array}$ \\
\hline Yeast GCN5 & 00) & VRNTSNIKYFLTYADNYAIGYFKKQGFTKEITLDKSIWMGYIKDYEGGTLMQCSMLPRIRYLDAGKILLLQEAALRRKI- \\
\hline Human GCN5 & 41) & ILYFLTYADEYAIGYFKKQGFSKDIKVPKSRYLGYIKDYE GATLME CELNPR IPYTELSHIIKKQKEIIKKLIE \\
\hline AtHAG1 (GCN5) & 16) & ARDVDGLTHFLTYADNNAVGYFVKQGFTKEIYLE KDVWHGFIKDYDGGLIMECKIDPKLPYTDLSSMIRQQRKAIDERI- \\
\hline AtHAG4 (HAM1) & (0) & 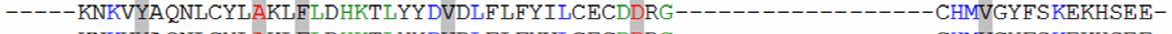 \\
\hline tHAG5 ( HAM2) & 50) & -----KNKVYAQNLCYLAKLFLDHKTLYYDVDLFLFYVLCECDDRG-------------------CHMVGYFSKEKHSEE- \\
\hline Consensus & $(321)$ & 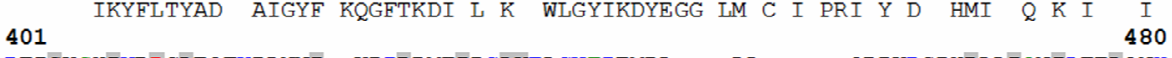 \\
\hline Yeast GCN5 & 79) & RTISKSHIVRPGLEQFKDLNNI--KPIDPMTIPGLKEAGWTPEMDA----LA-------QRPKRG PHDAAIQNILTELQNH \\
\hline Human GCN5 & 20) & RKQAQIRKVYPGLSCFKEGVRQ----IPVESVPGIRETGWKPLGKE---------KGKELKDPDQLYTTLKNLLAQIKSH \\
\hline AtHAG1 (GCN5) & 95) & RELSNCQNVYPKIEFLKNEAGIPRKI IKVEEIRGLREAGWTPDQWGHTRFKL FNGSADMVTNQKQLNALMRALLKTMQDH \\
\hline AtHAG4 (HAM1) & 6) & AYNLACILTLP PYQRKGYGKFL I AFSYELSKK------EGKVGTPERPLSDLGLVSYRGYWTRI LLDILKKHKGNISI KE \\
\hline AtHAG5 (HAM2) & 6) & AYNLACILTLPSYQRKGYGKFLIAFSYELSKK------EGKVGTPERPLSDLGLLSYRGYWTRVLLEILKKHKGNISI KE \\
\hline Consensus & $(401)$ & 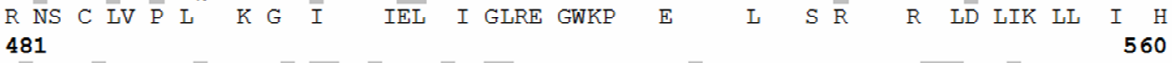 \\
\hline Yeast GCN5 & 47) & AAAWP FLQPVNKEEVPDYYDFIKEPMDLSTMEIKLESNKYQKMED-FIYDARLVFNNCRMYNGENTSYYKYANRLEKF FN \\
\hline Human GCN5 & 3) & PSAWP FMEPVKKSEAPDYYEVIRFPIDLKTMTERLRS-RYYVTRKL FVADLQRVIANCREYNPPDSEYCRCASALEKF FY \\
\hline AtHAG1 (GCN5) & 75) & ADAWP FKEPVD SRDVPDYYDI I KDPIDLKVIAKRVESEQYYVTLDMFVADARRMFNNCRTYNSPDTIYYKCATRLETHFH \\
\hline AtHAG4 (HAM1) & 380) & LSDMTAIKAED ILSTLQSLELIQYRKGQHVICAD PKVLDRHLKAAGRGGLDVDVSKMIWTPYKEQS------ \\
\hline AtHAG5 ( HAM2) & $(380)$ & LSDVTAIKAED ILSTLQSLELIQYRKGQHVICAD PKVLDRHLKAAGRGGLDVDASKLIWTPYKDQS------- \\
\hline Consensus & $(481)$ & E PDYYELIKYPIDL VI RLKS YHL \\
\hline Yeast GCN5 & 26) & $\begin{array}{l}561 \\
\text { NKVKE IPEYSHLID }\end{array}$ \\
\hline Human GCN5 & & FKLI \\
\hline HAG1 (GCN5) & & SKVQ \\
\hline LHAG4 (HAM1) & 46) & \\
\hline $\begin{array}{l}\text { THAG5 (HAM2) } \\
\text { Consensus }\end{array}$ & $\begin{array}{l}(446) \\
(561)\end{array}$ & \\
\hline
\end{tabular}

Supplemental Figure 1: Protein sequence alignment of Arabidopsis homologs of the yeast and human histone acetyltransferase GCN5. The amino acid (aa) sequences of the yeast GCN5, human GCN5, and the Arabidopsis HAG1 (AtGCN5), HAG4 and HAG5 proteins are aligned and are presented in the single letter code according to IUPAC nomenclature. Coloured letters indicate aa identities between two (green), three (blue) or all aligned proteins (red). Grey highlighted columns denote aa with similar biochemical properties. The black boxed amino acids are described to be crucial for HAT activity. The highly conserved glutamate, which is surrounded by several unpolar amino acids, deprotonates the e-amino function of the histone lysine side chain and enables a nucleophilic attack on the neighboring thioester function of acetyl-CoA (Biel et al., 2004). The resulting intermediate is stabilized by a hydrogen bond to the backbone amide of the close cysteine (present in yeast, human and Arabidopsis GCN5) or likely also the structural similar serine (present in HAG4 and HAG5). The intermediate decomposes, giving the general base glutamate, the acetylated histone and the reduced coenzyme A (Biel et al., 2004). According to the model of induced fit, the GCN5 HAT inhibitor y-butyrolacton was designed, which non-irreversibly binds the active site. 
AtUBQ5 qRT-PCR data

\begin{tabular}{|l|c|}
\multicolumn{1}{c}{ Ct-values } \\
\hline mock & 20,65 \\
+ TSA & 20,43 \\
+ NAA & 19,94 \\
+ NAA + TSA & 20,72 \\
\hline
\end{tabular}

\begin{tabular}{|l|c|}
\multicolumn{1}{c}{ Ct-values } \\
\hline mock & 19,60 \\
+ butyrolacton & 19,78 \\
+ NAA & 19,49 \\
+ NAA+ butyrolacton & 20,40 \\
\hline
\end{tabular}

Supplemental figure 2: Expression of the Ubiquitin5 gene is unaffected by auxin and the applied HAT and HDAC inhibitors. Expression analysis of the ubiquitin5 (UBQ5) gene in the absence or presence of auxin and the histone deacetylase (HDAC) inhibitor, Trichostatin A (TSA) or the GCN5 histone acetyltransferase (HAT) inhibitor, ybutyrolacton. To ensure equal exposure to the additives, mesophyll protoplasts were prepared and incubated for 3 hours in an isotonic incubation buffer supplemented with or without $0.8 \mu \mathrm{M}$ TSA or $0.25 \mathrm{mM}$-butyrolacton before protoplasts were subjected to an additional NAA $(2 \mu \mathrm{M})$ application for further 3 hours. UBQ5 expression was quantified by qRT-PCR and is presented as Ct-values. Given are mean Ct-values from four technical replicates from one out of three independent experiments.

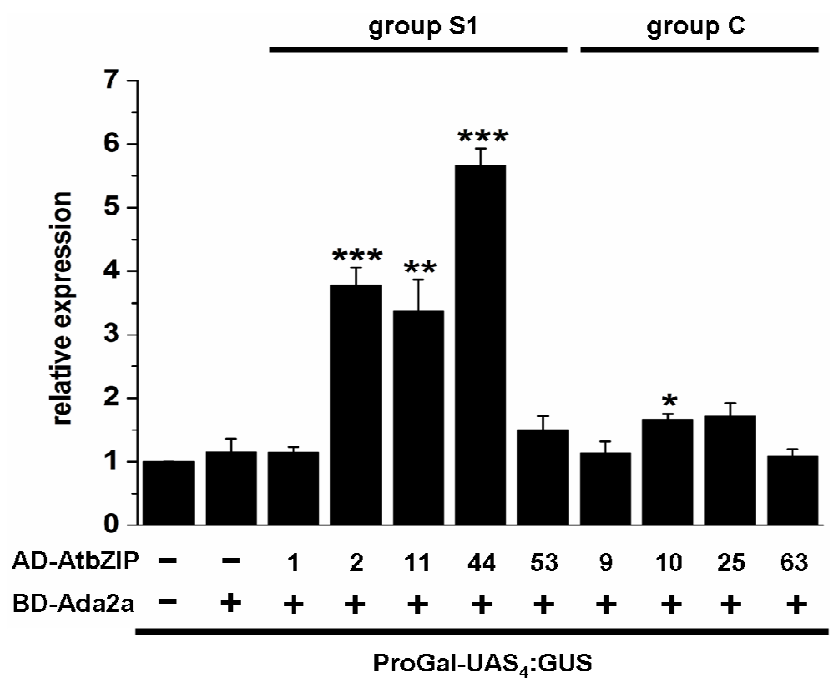

Supplemental figure 3: AtADA2a binding properties of group C and S1 AtbZIP-TFs. Group S1, AtbZIP11 related TFs and group C AtbZIP10 are able to bind AtADA2a. Binding properties were determined in P2H assays. Given are the mean relative expression levels $( \pm$ SEM) from 3 independent experiments with each of 2 technical replicates. Reporter gene expression was normalized to the expression of the Pro35S:NAN normalization construct and is relative to the expression of the Gal4-UAS promoter. Significant differences compared to the expression of the Gal4UAS:GUS reporter gene driven by BD-Ada2a had been determined by Students T-Test and are labeld with asterisks $(\mathrm{p} \leq 0,05=* ; \mathrm{p} \leq 0,01=* * ; \mathrm{p} \leq 0,001=* * *)$. 

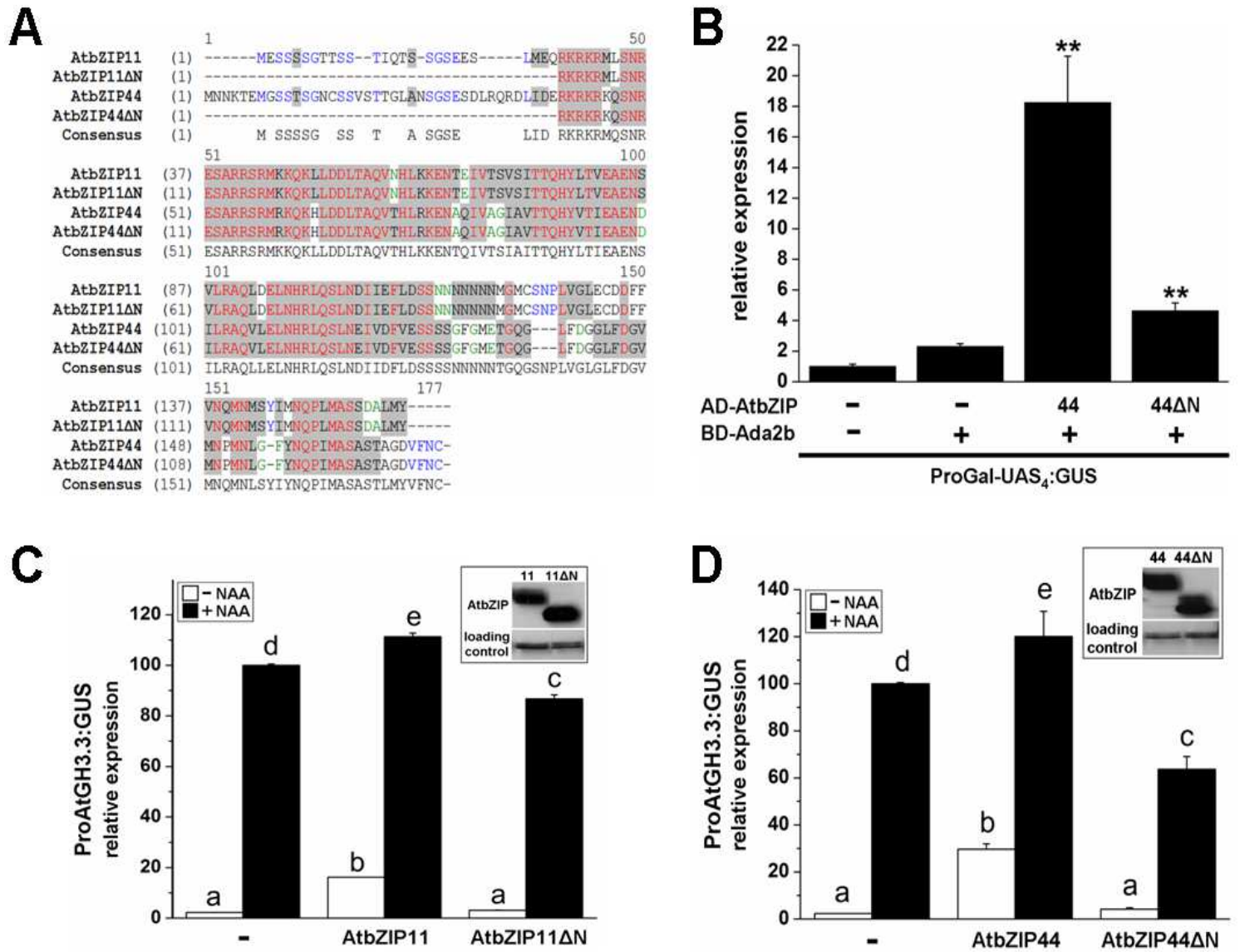

Supplemental figure 4: The acidic, polar N-terminus of AtbZIP11 related TFs is an activation domain and is crucial for AtADA2b binding. A) Alignment of the amino acid (aa) sequence of AtbZIP11 and AtbZIP44 TFs and their Nterminally truncated derivates. The closely related AtbZIP11 and AtbZIP44 share a high aa sequence similarity. AA sequence is presented in the single letter code according to IUPAC nomenclature. Coloured letters indicate amino acid identities between two (green), three (blue) or all aligned proteins (red). Grey highlighted columns denote aa with similar biochemical properties. B) The AtADA2b binding capacity of the full-length and N-terminal truncated AtbZIP44-TF protein were analysed in P2H assays. Although the full-length and N-terminally truncated HA-tagged proteins were equally expressed and stable in protoplasts, the $\mathrm{N}$-terminal truncated derivative shows, compared to the full-length protein an impaired capacity to bind AtADA2b. The activation properties of AtbZIP 11 (C) and AtbZIP44 (D) and their N-terminal truncated derivatives were analyses in protoplast transactication assays. Whereas both fulllength proteins are able to promote expression of the auxin responsive AtGH3.3 promoter in the absence (white bars) and presence (black bars) of exogenously applied auxin, the truncated proteins fail to activate the promoter and even suppress it in the presence of $0.25 \mu \mathrm{M}$ NAA. The presented results were obtained from 3 independent transfections with each of 2 technical replicates and are given as mean relative expression values $( \pm \mathrm{SD})$. Expression was normalized to the Pro35S:NAN normalization construct and are relative to the expression of (B) the ProGalUAS4:GUS reporter or (C, D) the auxin induced ProAtGH3.3:GUS construct (100\%). In the experiments listed above significant differences compared to $(\mathbf{B})$ the expression of the BD-ADA2b were determined by Students T-Test and are designated with asterisks ( $\mathrm{p} \leq 0.05=* ; \mathrm{p} \leq 0.01=* * ; \mathrm{p} \leq 0.001=* * *)$, whereas significant differences between $(\mathbf{C}, \mathbf{D})$ treatments and constructs were defined by one-way ANOVA and subsequent Fisher posthoc test $(\mathrm{p} \leq 0,05)$ and are visualized by different letters. 
A

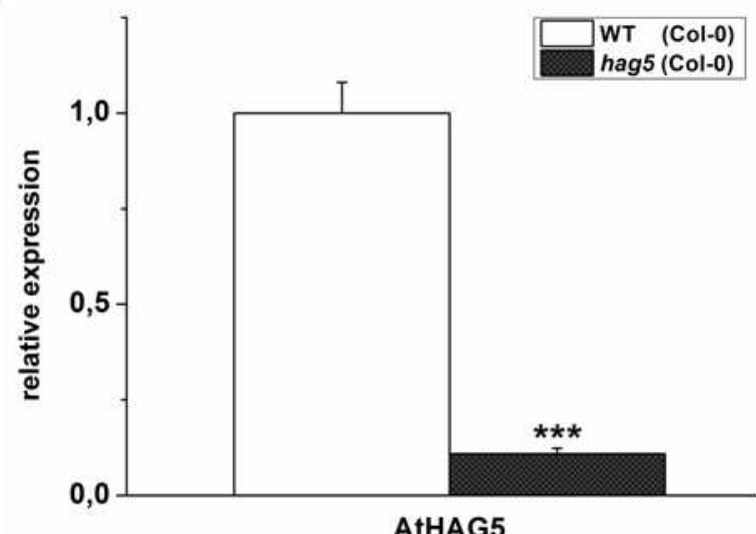

B

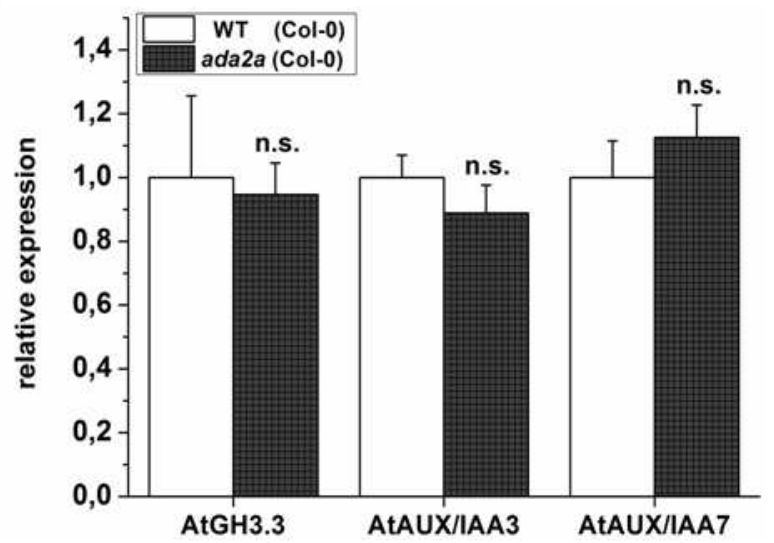

Supplemental Figure 5: Expression analysis of AtHAG5 in homozygous hag5 and of auxin responsive genes in ada2a mutant plants. A) AtHAG5 expression in homozygous hag5 mutant plants was determined by qRT-PCR. Given results were obtained from 5 individual WT and hag5 plants and are presented as mean expression levels $( \pm$ SEM) relative to the WT expression. B) Expression of the auxin responsive AtGH3.3, AtAUX/IAA3 and AtAUX/IAA7 genes was quantified by qRT-PCR in homozygous ada2a mutant plants. Given are mean relative expression levels ( \pm SEM) from 3 individual plants with each of 4 technical replicates. Given expression levels are relative to the corresponding WT. Significant differences between WT and mutants are defined by Students T-Test and are marked with asterisks $(\mathrm{p} \leq 0.05=* ; \mathrm{p} \leq$ $0.01=* * ; \mathrm{p} \leq 0.001=* * *)$. 


\section{References}

Alonso R, Onate-Sanchez L, Weltmeier F, Ehlert A, Diaz I, Dietrich K, Vicente-Carbajosa J, Droge-Laser W. 2009. A pivotal role of the basic leucine zipper transcription factor bZIP53 in the regulation of Arabidopsis seed maturation gene expression based on heterodimerization and protein complex formation. Plant Cell, 21: 1747.

Anzola JM, Sieberer T, Ortbauer M, Butt H, Korbei B, Weinhofer I, Mullner AE, Luschnig C. 2010. Putative Arabidopsis transcriptional adaptor protein (PROPORZ1) is required to modulate histone acetylation in response to auxin. Proc Natl Acad Sci U S A, 107: 10308.

Baena-Gonzalez E, Rolland F, Thevelein JM, Sheen J. 2007. A central integrator of transcription networks in plant stress and energy signalling. Nature, 448: 938.

Barlev NA, Candau R, Wang L, Darpino P, Silverman N, Berger SL. 1995. Characterization of physical interactions of the putative transcriptional adaptor, ADA2, with acidic activation domains and TATA-binding protein. J Biol Chem, 270: 19337.

Benhamed M, Bertrand C, Servet C, Zhou DX. 2006. Arabidopsis GCN5, HD1, and TAF1/HAF2 interact to regulate histone acetylation required for light-responsive gene expression. Plant Cell, 18 : 2893.

Benhamed M, Martin-Magniette ML, Taconnat L, Bitton F, Servet C, De Clercq R, De Meyer B, Buysschaert C, Rombauts S, Villarroel R, Aubourg S, Beynon J, Bhalerao RP, Coupland G, Gruissem W, Menke FL, Weisshaar B, Renou JP, Zhou DX, Hilson P. 2008. Genome-scale Arabidopsis promoter array identifies targets of the histone acetyltransferase GCN5. Plant J, 56: 493.

Bertrand C, Bergounioux C, Domenichini S, Delarue M, Zhou DX. 2003. Arabidopsis histone acetyltransferase AtGCN5 regulates the floral meristem activity through the WUSCHEL/AGAMOUS pathway. J Biol Chem, 278: 28246.

Bhat RA, Borst JW, Riehl M, Thompson RD. 2004. Interaction of maize Opaque-2 and the transcriptional co-activators GCN5 and ADA2, in the modulation of transcriptional activity. Plant Mol Biol, 55: 239.

Bhat RA, Riehl M, Santandrea G, Velasco R, Slocombe S, Donn G, Steinbiss HH, Thompson RD, Becker HA. 2003. Alteration of GCN5 levels in maize reveals dynamic responses to manipulating histone acetylation. Plant J, 33: 455.

Biel M, Kretsovali A, Karatzali E, Papamatheakis J, Giannis A. 2004. Design, synthesis, and biological evaluation of a small-molecule inhibitor of the histone acetyltransferase Gcn5. Angew Chem Int Ed Engl, 43: 3974.

Cecchetti V, Altamura MM, Falasca G, Costantino P, Cardarelli M. 2008. Auxin regulates Arabidopsis anther dehiscence, pollen maturation, and filament elongation. Plant Cell, 20: 1760.

Clough SJ, Bent AF. 1998. Floral dip: a simplified method for Agrobacterium-mediated transformation of Arabidopsis thaliana. Plant J, 16: 735.

Dharmasiri N, Dharmasiri S, Estelle M. 2005. The F-box protein TIR1 is an auxin receptor. Nature, 435: 441.

Dietrich K WF, Ehlert A, Weiste C, Stahl M, Harter K, Dröge-Laser W. 2011. Heterodimers of the Arabidopsis Transcription Factors bZIP1 and bZIP53 are Reprogramming Amino Acid Metabolism during Low Energy Stress. Plant Cell, in press.

Earley KW, Shook MS, Brower-Toland B, Hicks L, Pikaard CS. 2007. In vitro specificities of Arabidopsis co-activator histone acetyltransferases: implications for histone hyperacetylation in gene activation. Plant J, 52: 615.

Ehlert A, Weltmeier F, Wang X, Mayer CS, Smeekens S, Vicente-Carbajosa J, Droge-Laser W. 2006. Two-hybrid protein-protein interaction analysis in Arabidopsis protoplasts: establishment of a heterodimerization map of group C and group S bZIP transcription factors. Plant J, 46: 890.

Fode B, Gatz C. 2009. Chromatin immunoprecipitation experiments to investigate in vivo binding of Arabidopsis transcription factors to target sequences. Methods Mol Biol, 479: 261.

Gray WM, Kepinski S, Rouse D, Leyser O, Estelle M. 2001. Auxin regulates SCF(TIR1)-dependent degradation of AUX/IAA proteins. Nature, 414: 271.

Guilfoyle TJ, Hagen G. 2007. Auxin response factors. Curr Opin Plant Biol, 10: 453. 
Hanson J, Hanssen M, Wiese A, Hendriks MM, Smeekens S. 2008. The sucrose regulated transcription factor bZIP11 affects amino acid metabolism by regulating the expression of ASPARAGINE SYNTHETASE1 and PROLINE DEHYDROGENASE2. Plant J, 53: 935.

Hark AT, Vlachonasios KE, Pavangadkar KA, Rao S, Gordon H, Adamakis ID, Kaldis A, Thomashow MF, Triezenberg SJ. 2009. Two Arabidopsis orthologs of the transcriptional coactivator ADA2 have distinct biological functions. Biochim Biophys Acta, 1789: 117.

Jakoby M, Weisshaar B, Droge-Laser W, Vicente-Carbajosa J, Tiedemann J, Kroj T, Parcy F. 2002. bZIP transcription factors in Arabidopsis. Trends Plant Sci, 7: 106.

Kepinski S, Leyser O. 2005. The Arabidopsis F-box protein TIR1 is an auxin receptor. Nature, 435: 446.

Kirby J, Kavanagh TA. 2002. NAN fusions: a synthetic sialidase reporter gene as a sensitive and versatile partner for GUS. Plant J, 32: 391.

Kornet N, Scheres B. 2009. Members of the GCN5 histone acetyltransferase complex regulate PLETHORA-mediated root stem cell niche maintenance and transit amplifying cell proliferation in Arabidopsis. Plant Cell, 21: 1070.

Latrasse D, Benhamed M, Henry Y, Domenichini S, Kim W, Zhou DX, Delarue M. 2008. The MYST histone acetyltransferases are essential for gametophyte development in Arabidopsis. $B M C$ Plant Biol, 8: 121.

Lee KK, Workman JL. 2007. Histone acetyltransferase complexes: one size doesn't fit all. Nat Rev Mol Cell Biol, 8: 284.

Liu Z, Karmarkar V. 2008. Groucho/Tup1 family co-repressors in plant development. Trends Plant Sci, 13: 137.

Locatelli S, Piatti P, Motto M, Rossi V. 2009. Chromatin and DNA modifications in the Opaque2mediated regulation of gene transcription during maize endosperm development. Plant Cell, 21: 1410.

Long JA, Ohno C, Smith ZR, Meyerowitz EM. 2006. TOPLESS regulates apical embryonic fate in Arabidopsis. Science, 312: 1520.

Lusser A, Kolle D, Loidl P. 2001. Histone acetylation: lessons from the plant kingdom. Trends Plant Sci, 6: 59.

Mao Y, Pavangadkar KA, Thomashow MF, Triezenberg SJ. 2006. Physical and functional interactions of Arabidopsis ADA2 transcriptional coactivator proteins with the acetyltransferase GCN5 and with the cold-induced transcription factor CBF1. Biochim Biophys Acta, 1759: 69.

Mosley AL, Pattenden SG, Carey M, Venkatesh S, Gilmore JM, Florens L, Workman JL, Washburn MP. 2009. Rtr1 is a CTD phosphatase that regulates RNA polymerase II during the transition from serine 5 to serine 2 phosphorylation. Mol Cell, 34: 168.

Nakagawa T, Kurose T, Hino T, Tanaka K, Kawamukai M, Niwa Y, Toyooka K, Matsuoka K, Jinbo T, Kimura T. 2007. Development of series of gateway binary vectors, pGWBs, for realizing efficient construction of fusion genes for plant transformation. J Biosci Bioeng, 104: 34.

Navarro L, Dunoyer P, Jay F, Arnold B, Dharmasiri N, Estelle M, Voinnet O, Jones JD. 2006. A plant miRNA contributes to antibacterial resistance by repressing auxin signaling. Science, 312: 436.

Nemhauser JL, Mockler TC, Chory J. 2004. Interdependency of brassinosteroid and auxin signaling in Arabidopsis. PLoS Biol, 2: E258.

Pandey R, Muller A, Napoli CA, Selinger DA, Pikaard CS, Richards EJ, Bender J, Mount DW, Jorgensen RA. 2002. Analysis of histone acetyltransferase and histone deacetylase families of Arabidopsis thaliana suggests functional diversification of chromatin modification among multicellular eukaryotes. Nucleic Acids Res, 30: 5036.

Pauwels L, Barbero GF, Geerinck J, Tilleman S, Grunewald W, Perez AC, Chico JM, Bossche RV, Sewell J, Gil E, Garcia-Casado G, Witters E, Inze D, Long JA, De Jaeger G, Solano R, Goossens A. 2010. NINJA connects the co-repressor TOPLESS to jasmonate signalling. Nature, 464: 788.

Paz-Ares J. 2002. REGIA, an EU project on functional genomics of transcription factors from Arabidopsis Thaliana. Comp Funct Genomics, 3: 102.

Pufky J, Qiu Y, Rao MV, Hurban P, Jones AM. 2003. The auxin-induced transcriptome for etiolated Arabidopsis seedlings using a structure/function approach. Funct Integr Genomics, 3: 135. 
Sambrook FaM. 1989. Molecular cloning: A laboratory manual. Cold spring Harbor Laboratory Press.

Schmitz D, Lohmer S, Salamini F, Thompson RD. 1997. The activation domain of the maize transcription factor Opaque-2 resides in a single acidic region. Nucleic Acids Res, 25: 756.

Servet C, Conde e Silva N, Zhou DX. 2010. Histone acetyltransferase AtGCN5/HAG1 is a versatile regulator of developmental and inducible gene expression in Arabidopsis. Mol Plant, 3: 670.

Shahbazian MD, Grunstein M. 2007. Functions of site-specific histone acetylation and deacetylation. Annu Rev Biochem, 76: 75.

Shan L, He P, Li J, Heese A, Peck SC, Nurnberger T, Martin GB, Sheen J. 2008. Bacterial effectors target the common signaling partner BAK1 to disrupt multiple MAMP receptor-signaling complexes and impede plant immunity. Cell Host Microbe, 4: 17.

Sheen J. 2001. Signal transduction in maize and Arabidopsis mesophyll protoplasts. Plant Physiol, 127: 1466.

Sieberer T, Hauser MT, Seifert GJ, Luschnig C. 2003. PROPORZ1, a putative Arabidopsis transcriptional adaptor protein, mediates auxin and cytokinin signals in the control of cell proliferation. Curr Biol, 13: 837.

Skoog Ma. 1962. A revised medium for rapid growth and bioassays with tobacco tissue cultures. Physiol Plant, 15: 473.

Stockinger EJ, Mao Y, Regier MK, Triezenberg SJ, Thomashow MF. 2001. Transcriptional adaptor and histone acetyltransferase proteins in Arabidopsis and their interactions with CBF1, a transcriptional activator involved in cold-regulated gene expression. Nucleic Acids Res, 29: 1524.

Swaminathan K, Peterson K, Jack T. 2008. The plant B3 superfamily. Trends Plant Sci, 13: 647.

Szemenyei H, Hannon M, Long JA. 2008. TOPLESS mediates auxin-dependent transcriptional repression during Arabidopsis embryogenesis. Science, 319: 1384.

Tiwari SB, Hagen G, Guilfoyle TJ. 2004. Aux/IAA proteins contain a potent transcriptional repression domain. Plant Cell, 16: 533.

Ulmasov T, Hagen G, Guilfoyle TJ. 1997. ARF1, a transcription factor that binds to auxin response elements. Science, 276: 1865.

Ulmasov T, Hagen G, Guilfoyle TJ. 1999a. Activation and repression of transcription by auxinresponse factors. Proc Natl Acad Sci U S A, 96: 5844.

Ulmasov T, Hagen G, Guilfoyle TJ. 1999b. Dimerization and DNA binding of auxin response factors. Plant J, 19: 309.

Ulmasov T, Liu ZB, Hagen G, Guilfoyle TJ. 1995. Composite structure of auxin response elements. Plant Cell, 7: 1611.

Vlachonasios KE, Thomashow MF, Triezenberg SJ. 2003. Disruption mutations of ADA2b and GCN5 transcriptional adaptor genes dramatically affect Arabidopsis growth, development, and gene expression. Plant Cell, 15: 626.

Waltner JK, Peterson FC, Lytle BL, Volkman BF. 2005. Structure of the B3 domain from Arabidopsis thaliana protein At1g16640. Protein Sci, 14: 2478.

Wang D, Pajerowska-Mukhtar K, Culler AH, Dong X. 2007. Salicylic acid inhibits pathogen growth in plants through repression of the auxin signaling pathway. Curr Biol, 17: 1784.

Weiste C, Iven T, Fischer U, Onate-Sanchez L, Droge-Laser W. 2007. In planta ORFeome analysis by large-scale over-expression of GATEWAY-compatible cDNA clones: screening of ERF transcription factors involved in abiotic stress defense. Plant J, 52: 382.

Weiste CaD-LW. G-box related cis-Elements and their cognate bZIP Transcription Factors function as quantitative Modulators of Auxin-mediated growth responses

Woodward AW, Bartel B. 2005. Auxin: regulation, action, and interaction. Ann Bot, 95: 707.

Yoshida M, Kijima M, Akita M, Beppu T. 1990. Potent and specific inhibition of mammalian histone deacetylase both in vivo and in vitro by trichostatin A. J Biol Chem, 265: 17174.

Zhao Y. 2010. Auxin biosynthesis and its role in plant development. Annu Rev Plant Biol, 61: 49.

Zuo J, Niu QW, Chua NH. 2000. Technical advance: An estrogen receptor-based transactivator XVE mediates highly inducible gene expression in transgenic plants. Plant J, 24: 265. 


\title{
3.4 Chapter 4: Heterodimers of the Arabidopsis transcription factors bZIP1 and bZIP53 reprogram amino acid metabolism during low energy stress
}

\section{Heterodimers of the Arabidopsis Transcription Factors bZIP1 and bZIP53 Reprogram Amino Acid Metabolism during Low Energy Stress ${ }^{\text {}}$}

\author{
Katrin Dietrich,, ${ }^{a, b, 1}$ Fridtjof Weltmeier, ${ }^{b, 1}$ Andrea Ehlert, ${ }^{b}$ Christoph Weiste, ${ }^{\text {a,b }}$ Mark Stahl,c ${ }^{c}$ Klaus Harter, ${ }^{c}$ and \\ Wolfgang Dröge-Lasera,b,2 \\ a Julius-von-Sachs-Institut, Pharmazeutische Biologie, Universität Würzburg, Julius-von-Sachs-Platz 2, D-97082 Wuerzburg, \\ Germany \\ b Albrecht-von-Haller Institut, Universität Göttingen, D-37073 Gottingen, Germany \\ c Zentrum für Molekularbiologie der Pflanzen, Pflanzenphysiologie, 72076 Tuebingen, Germany
}

Control of energy homeostasis is crucial for plant survival, particularly under biotic or abiotic stress conditions. Energy deprivation induces dramatic reprogramming of transcription, facilitating metabolic adjustment. An in-depth knowledge of the corresponding regulatory networks would provide opportunities for the development of biotechnological strategies. Low energy stress activates the Arabidopsis thaliana group S1 basic leucine zipper transcription factors bZIP1 and bZIP53 by transcriptional and posttranscriptional mechanisms. Gain-of-function approaches define these bZIPs as crucial transcriptional regulators in Pro, Asn, and branched-chain amino acid metabolism. Whereas chromatin immunoprecipitation analyses confirm the direct binding of bZIP1 and bZIP53 to promoters of key metabolic genes, such as ASPARAGINE SYNTHETASE1 and PROLINE DEHYDROGENASE, the G-box, C-box, or ACT motifs (ACTCAT) have been defined as regulatory cis-elements in the starvation response. bZIP1 and bZIP53 were shown to specifically heterodimerize with group C bZIPs. Although single loss-of-function mutants did not affect starvation-induced transcription, quadruple mutants of group S1 and C bZIPs displayed a significant impairment. We therefore propose that bZIP1 and bZIP53 transduce low energy signals by heterodimerization with members of the partially redundant $\mathrm{C} / \mathrm{S} 1 \mathrm{bZIP}$ factor network to reprogram primary metabolism in the starvation response.

\section{INTRODUCTION}

Due to their phototrophic life style, plants have to steadily adjust their metabolism to day-night rhythms and environmental changes to withstand transient energy deprivation (reviewed in Baena-González and Sheen, 2008; Usadel et al., 2008). Low energy stress can easily be mimicked by the cultivation of plants in the dark. Extended dark treatment is correlated with dramatic changes in primary plant metabolism, in particular, reduced photosynthesis, degradation of proteins, amino acids, or nucleic acids, hydrolysis of polysaccharides, or oxidation of fatty acids. These physiological changes are accompanied by a massive reprogramming of transcription, which is reflected in several recent transcriptome profiling studies (Gan, 2003; Lin and Wu, 2004; Buchanan-Wollaston et al., 2005) In particular, the whole set of genes leading to the biosynthesis of Asn is reprogrammed in response to dark treatment. In comparison to Gln, the major transport form of nitrogen in the light, Asn, contains less carbon than GIn and is therefore used to store and transport nitrogen

${ }^{1}$ These authors contributed equally to this work.

${ }^{2}$ Address correspondence to wolfgang.droege-laser@uni-wuerzburg de.

The author responsible for distribution of materials integral to the findings presented in this article in accordance with the policy described in the Instructions for Authors (www.plantcell.org) is: Wolfgang DrögeLaser (wolfgang.droege-laser@uni-wuerzburg.de).

WOnline version contains Web-only data.

www.plantcell.org/cgi/doi/10.1105/tpc. 110.075390 especially under stress conditions where carbon is limited (Lam et al., 1994).

Recently, the Arabidopsis thaliana SnRK1-like kinases (SNF1related protein kinases 1) KIN10 and KIN11 have been proposed to function as central signaling integrators that mediate adaptation to low energy stress (Baena-González et al., 2007). These kinases show structural similarities to the SNF1 kinase (for SUCROSE NONFERMENTING1) in yeast and the AMP-DEPENDENT PROTEIN KINASE in mammals, which function as master regulators of the energy balance that is essential for survival under stress (Polge and Thomas, 2007). In Arabidopsis, the ASPARAGINE SYNTHETASE1 (ASN1) gene, which encodes the final step in Asn biosynthesis, was proposed to be a target of the KIN10/11 pathway that regulates the level of Asn (BaenaGonzález et al., 2007). The dark- or stress-induced regulation of ASN1 expression is mediated specifically by a G-box ciselement (Baena-González et al., 2007; Hanson et al., 2008), which is typically recognized by basic leucine zipper (bZIP) transcription factors (TFs). bZIP proteins, which are exclusively found in eukaryotic cells, bind DNA by forming homo- or heterodimers. In the Arabidopsis genome, 75 bZIP genes have been identified and classified into 10 groups (Jakoby et al., 2002). Interestingly, only a specific subset of G-box binding bZIP factors was shown to activate ASN1 in transiently transformed protoplasts (BaenaGonzález et al., 2007), namely, bZIP2 (GBF5), bZIP11 (ATB2), bZIP53, and bZIP1. Based on amino acid homology and specific heterodimerization properties with group $\mathrm{C}$ bZIPs, these proteins were classified as belonging to the S1 subgroup (Ehlert et al., 
2006). These $\mathrm{C}$ and S1 bZIPs form a functional interlinked TF network (Weltmeier et al., 2009).

In a transcriptome analysis using plants expressing bZIP11 in a dexamethasone-inducible manner, ASN1 was shown to be regulated by bZIP11 (Hanson et al., 2008). Furthermore, bZIP53 regulates the expression of PROLINE DEHYDROGENASE (ProDH) during the hypoosmolarity response (Nakashima et al., 1998; Satoh et al., 2004; Weltmeier et al., 2006). ProDH degrades the compatible osmolyte Pro during recovery from stress.

To deal with diurnal changes in carbon supplies, plants retain some photosynthates as starch, which can be remobilized during the night (Usadel et al., 2008). However, within 2 to $4 \mathrm{~h}$ of an extended night, these resources are depleted, leading to severe limitation of carbohydrates. This metabolic process is demonstrated in the starchless pgm mutant from Arabidopsis, which uses up its carbohydrate resources within the first few hours of night, eventually leading to growth retardation (Usade et al., 2008). Expression profiling of plants cultivated in an extended night regime allowed the construction of regulatory models, and these models suggest that plants respond to small changes in the carbon status in an acclimatory manner (Usade et al., 2008). In this work, several group S1 bZIPs, including bZIP1 and bZIP53, were suggested to be involved in the plant's response to carbohydrate starvation. Accordingly, systems biology approaches studying the integration of $\mathrm{C}$ - and $\mathrm{N}$-derived metabolic signals proposed bZIP1 as a regulator in the nitrogenresponsive gene network, which includes the modulation of ASN1 expression (Gutiérrez et al., 2008).

Although several members of the group S1 bZIPs have been implicated in starvation responses and particularly in amino acid metabolism, experimental data are limited to gain-of-function studies in plant protoplasts. Here, we define bZIP1 and, to a mino extent, bZIP53 as transcriptionally and posttranscriptionally activated TFs in the low energy stress response of Arabidopsis. The impact of bZIP1 and bZIP53 on the starvation-induced transcription of key genes in amino acid metabolism and amino acid accumulation is demonstrated in protoplasts and transgenic plants. The results of loss-of-function approaches indicate that several partially redundant TFs of the C/S1 bZIP network cooperate to regulate plant low energy responses.

\section{RESULTS}

\section{bZIP1 and bZIP53 Expression Is Enhanced during} Dark-Induced Starvation

To identify candidate bZIP TFs, which are involved in regulating plant starvation responses, a screening of public expression databases (Hruz et al., 2008) and quantitative real-time PCR (qPCR) using RNA from plants exposed to extended darkness were performed. The expression of $b Z I P 1$ was strongly induced upon extended dark treatment and repressed by sugars (see Supplemental Figures $1 \mathrm{~A}$ to $1 \mathrm{C}$ online). A minor but reproducible transcriptional induction was also observed for the closest $b Z I P$ homolog, bZIP53, but not for the other group S1 bZIPs. Based on these findings, $b Z I P 1$ and $b Z I P 53$ were selected as candidate transcriptional regulators to study the dark-induced starvation response of Arabidopsis.
To further substantiate these findings, a detailed time-course expression experiment was performed. Remarkably, a night extension of up to $4 \mathrm{~h}$ leads to an eightfold accumulation of bZIP1 transcripts (Figure 1A), which further increased during an extended night by up to 30 -fold. bZIP53 transcripts accumulate only slightly during an extended night (three- to fourfold). Histochemical staining of plants containing promoter reporter constructs (ProbZIP1:GUS [for $\beta$-glucuronidase] and ProbZIP53: GUS) was used to demonstrate bZIP expression at the wholeplant level. Whereas the GUS staining of plants grown under a 16-h/8-h day-night cycle demonstrated a bZIP1 and bZIP53 gene activity only in young sink leaves (Weltmeier et al., 2009), the prolonged incubation in the dark led to a rapid spreading of expression patterns also into older, well-developed source leaves (Figure 1B). However, this response was not detected in transgenic lines harboring promoter:reporter constructs of other group S1 bZIPs (e.g., bZIP11 or bZIP44) (Weltmeier et al., 2009).

As demonstrated in previous studies (Wiese et al., 2004; Weltmeier et al., 2009), a posttranscriptional regulatory mechanism applies for all group S1 bZIPs, including bZIP1 and bZIP53, which leads to a sucrose-induced repression of translation mediated by a conserved system of upstream open reading frames (uORFs). With respect to bZIP1 and bZIP53, transcriptional and posttranscriptional mechanisms interact to enhance expression in response to dark treatment. By contrast, in particular bZIP11 shows an inverse regulation. Transcription is repressed by dark treatment and induced by sugar application (see Supplemental Figure 1B online). Hence, these differences in expression suggest a function for bZIP1 and bZIP53 in the darkinduced starvation response that is not shared by the other group S1 members.

\section{Ectopic Expression of $b Z I P 1$ and $b Z I P 53$ Results in Enhanced Dark-Induced Senescence}

To further study the function of bZIP1 and bZIP53 in the darkinduced starvation response, the phenotypes of plants ectopically expressing bZIP53 and bZIP1 (Pro35S:bZIP53 and Pro35S: $b Z I P 1)$ and their HA-tagged versions (Pro35S:HA-bZIP53 and Pro35S:HA-bZIP1) under the control of the 35S promoter were analyzed (Weltmeier et al., 2006, 2009; see Supplemental Figure 2 online). Whereas Pro35S:bZIP1 plants grew normally under standard day-night cycles, Pro35S:bZIP53 plants showed a dwarf growth phenotype that depended on the expression level of the transgene (Alonso et al., 2009). Prolonged cultivation of the bZIP overexpressing lines in the dark resulted in an obvious phenotype (Figure 1C). In particular, the Pro35S:bZIP1 plants showed a faster dark-induced leaf yellowing, which was less pronounced for Pro35S:bZIP53 plants. Accordingly, the bZIP1 and bZIP53 overexpressing plants had significantly reduced amounts of chlorophyll after $4 \mathrm{~d}$ of cultivation in darkness (Figure 1D). However, bzip1 and bzip53 single and double mutants (see Supplemental Figure 2 online) did not show obvious alterations in comparison to the wild type.

The culture conditions were further analyzed using welldefined marker genes for ongoing leaf senescence (see Supplemental Figure 3 online). Whereas the chlorophyll $a / b$ binding protein gene $(C A B)$, a light-induced marker for photosynthetically 


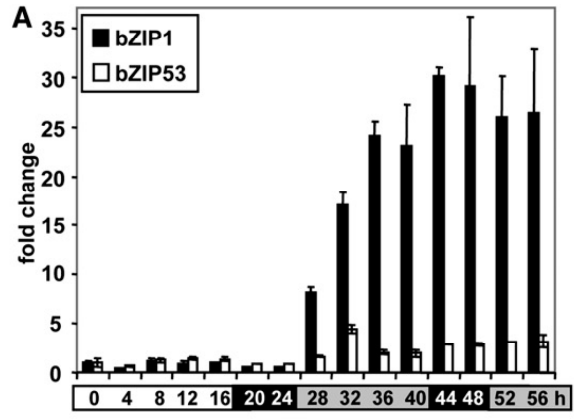

B
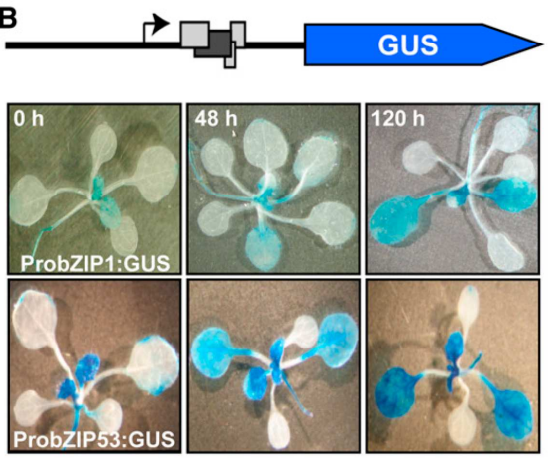

C
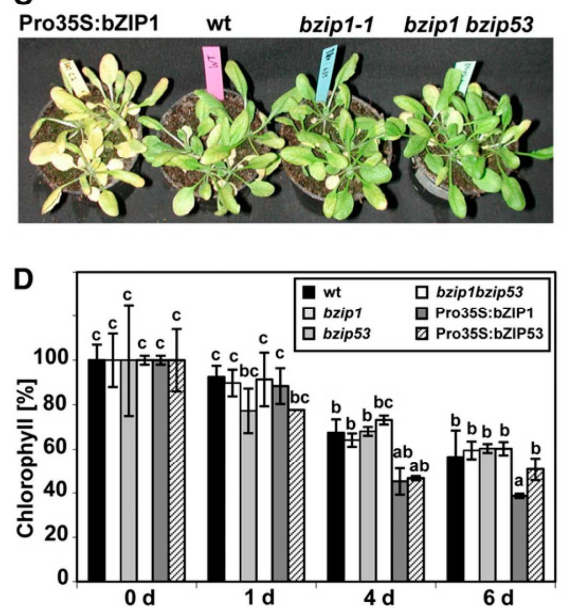

Figure 1. Analysis of bZIP1 and bZIP53 in the Low Energy Response.

(A) Expression of bZIP1 and bZIP53 increases after extended night treatment. Wild-type plants are cultivated at a day/night cycle of $16 / 8 \mathrm{~h}$ as indicated by the scheme. Day, night, and extended night phases are indicated by white, black, and gray bars, respectively. Transcript abundance as determined by APCR is presented for bZIP1 (black bars) and bZIP53 (white bars). Rosette leaves of 103 -week-old plants were pooled and used for RNA preparation and GPCR at the time points indicated. active leaves, was transcriptionally downregulated in darkness (van der Graaff et al., 2006), the SENESCENCE ASSOCIATED GENE103 (SAG103), a marker for dark-induced senescence, was induced in wild-type plants after $24 \mathrm{~h}$ of extended night (at the 48-h time point). By contrast, the YELLOW LEAF SPECIFIC3 (YLS3) gene, a marker for natural senescence (van der Graaff et al., 2006), was not significantly affected in its transcription. We therefore conclude that the process observed during extended night is distinct from natural senescence and that ectopic expression of $b Z I P 1$ or bZIP53 enhances physiological responses that are correlated to dark-induced starvation.

bZIP1 and bZIP53 Regulate ProDH Transcript Level and Pro Content during Dark-Induced Starvation

ProDH, which encodes an enzyme that mediates the catabolism of Pro (Figure 2A), is a direct transcriptional target of bZIP53 in the hypoosmolarity response of Arabidopsis (Weltmeier et al., 2006). ProDH transcription was also induced after dark treatment, as demonstrated by RNA gel blot analysis (Figure 2B) and qPCR (Figure 2C). These data are in agreement with the hypothesis that, during the starvation response, amino acids are recycled to support $\mathrm{C}, \mathrm{N}$, and energy demands. Ectopic expression of $b$ ZIP1 and $b$ ZIP53 resulted in significantly higher levels of ProDH transcripts. However, whereas bZIP53 overexpression led to high $P r o D H$ transcript levels both in light and darkness, which was further enhanced by extended dark treatment, the regulation by bZIP1 differed, as $P r o D H$ transcript accumulation was preferentially enhanced in the dark (Figures 2B and 2G).

In addition to an overexpression analysis, we also performed loss-of-function studies using T-DNA insertion mutants of $b Z I P 1$ and bZIP53 (see Supplemental Figures 2 and 4 online). In contrast with single bzip 1 and bzip53 mutants (see Supplemental Figure 5B online), a moderate but significant reduction in the dark-induced activation of $P r o D H$ transcript accumulation was observed in bzip1 bzip53 double mutants when compared with the wild type (Figure 2C; see Supplemental Figure 5B online). However, because the ProDH transcript level is still responsive to

Fold change compared with wild-type expression is depicted at $0 \mathrm{~h}$. Mean value and SD of two replicates are given.

(B) Histochemical GUS staining of transgenic plants expressing ProbZIP1:GUS (top panel) and ProbZIP53:GUS (bottom panel). The upstream regions of the constructs expressed in these plants (diagram above) contain the conserved system of uORFs (depicted by rectangles) that mediates sucrose-dependent posttranslational repression (Wiese et al., 2005; Weltmeier et al., 2009). GUS staining of plants grown under a $16 / 8$-h day/night cycle $(0 \mathrm{~h})$ or darkness for 48 and $120 \mathrm{~h}$ is shown.

(C) Three-week-old plants expressing bZIP1 under control of the $35 \mathrm{~S}$ promoter (Pro35S:bZIP1), the wild type (wt), bzip1, and the bzip1 bzip53 double mutant were analyzed for an enhanced senescence phenotype after $6 \mathrm{~d}$ in the dark.

(D) Relative chlorophyll content of rosette leaves of Pro35:bZIP53 and the plants depicted in (C). Plants were cultured in a normal day/nigh cycle (white bars) or for extended dark treatment as indicated. Significance was tested by one-way analysis of variance (ANOVA) analysis following Fisher's LSD post-test, $\mathrm{P}<0.05$. 
A

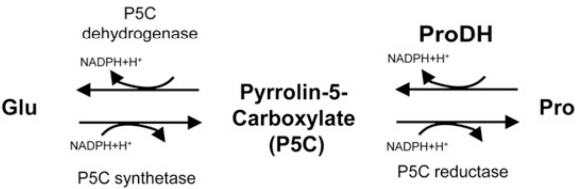

B
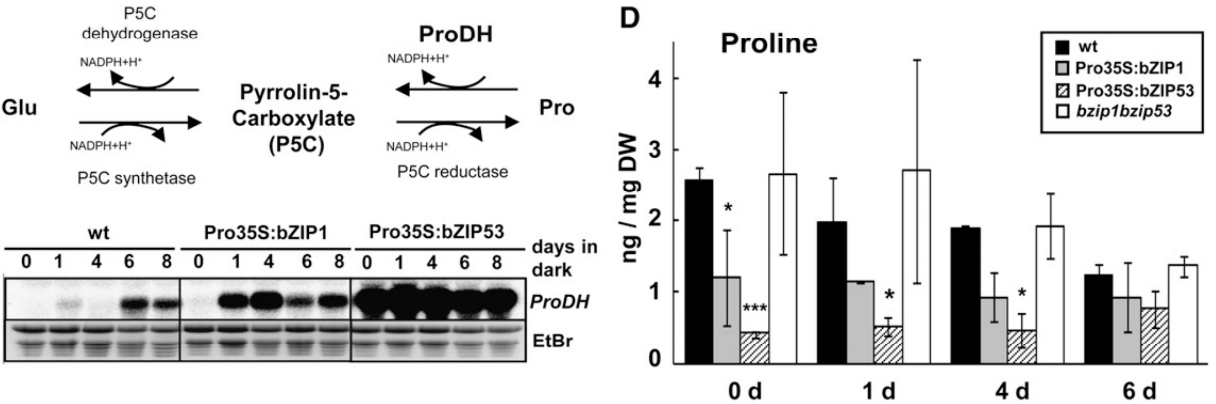

C

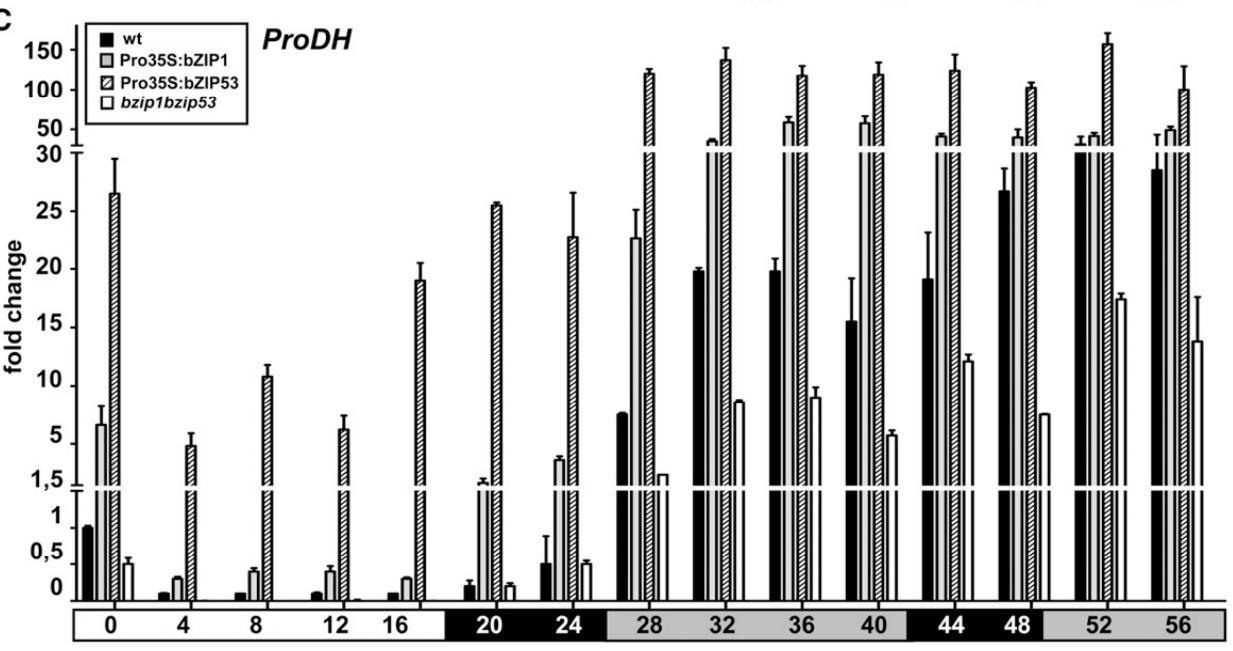

E

$24 \mathrm{~h}$

$+1 \mathrm{~h}$

$+2 \mathrm{~h}$

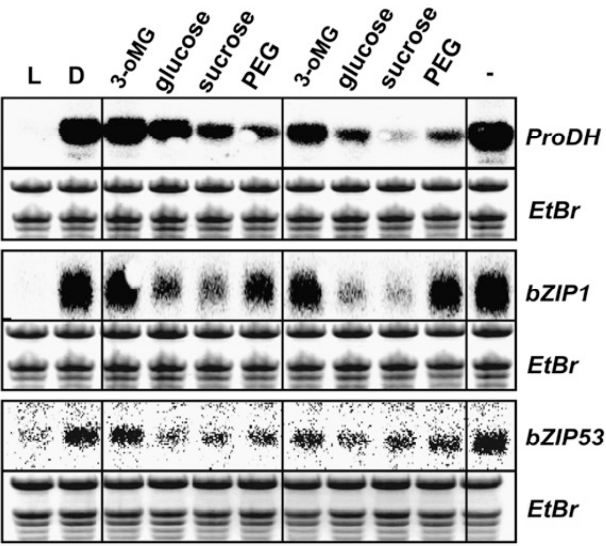

Figure 2. bZIP1 and bZIP53 Regulate ProDH Transcription and Pro Content during Dark Treatment.

(A) The ProDH enzyme regulates catabolism of the amino acid Pro to pyrrolin-5-carboxylate (P5C). Given is the complementary biosynthesis pathway based on Glu and making use of P5C as intermediate (Hellmann et al., 2000).

(B) RNA gel blot analysis of ProDH in the wild type (wt), Pro35S:bZIP1 (line C), and Pro35S:bZIP53 (line 10) (Weltmeier et al., 2006) in response to longterm dark treatment for 1 to $8 \mathrm{~d}$. Plant material was harvested late in the afternoon (5 PM). 
dark-induced starvation, additional, partly redundant, transcriptional regulators have to be postulated.

As the ProDH enzyme mediates Pro degradation, its activation should result in reduced Pro levels, which indeed was observed after transfer of wild-type plants to darkness (Figure 2D). Compared with the wild type and the bzip1 bzip53 double mutant, the Pro levels were significantly reduced in Pro35S:bZIP53 and Pro35S: bZIP1 plants (Figure 2D). This observation is in agreement with our postulated function of bZIP1 and bZIP53 in ProDH-mediated Pro degradation during the dark-induced starvation response.

\section{Dark-Induced $b Z I P 1$ Expression Depends on Sugar Depletion}

To elucidate whether depletion of sugars, which function as the major energy resource during night, or the absence of light function as important regulatory signals in the expression of bZIP1, bZIP53, and ProDH, we grew Arabidopsis plants in a hydroponic culture system under different carbohydrate regimes. As shown in Figure 2E, the transcripts of bZIP1, bZIP53, and $P r o D H$ coordinately accumulated after $24 \mathrm{~h}$ in the dark. However, transcriptional regulation of bZIP53 was always less pronounced when compared with bZIP1 (Figures 1A and 2E). The plants were then transferred to medium supplemented with equimolar concentrations of 3-ortho-methyl-glucose (3-oMG), glucose, sucrose, or polyethylene glycol (PEG) and further kept in darkness. 3-oMG serves as a control as it is taken up by the cells but does not trigger the glucose-specific sugar signaling pathways (Cortès et al., 2003). Sucrose and glucose, but not 3-oMG, repressed bZIP1, bZIP53, and ProDH transcript accumulation. These data suggest that sugar signaling and not the absence of light regulates the transcript accumulation of $b Z I P 1$ and bZIP53. As a putative target, the ProDH transcript level followed that of the two bZIP TFs, with a slower kinetic, as demonstrated by comparing the 1- and 2-h time points. It has been reported that changing of the osmolarity conditions also modulates the ProDH transcript levels (Satoh et al., 2004; Weltmeier et al., 2006). Hyperosmolarity conditions applied by PEG1000 treatment led to downregulation of ProDH but did not affect bZIP1 transcript accumulation. From these data, we conclude that, while ProDH transcript accumulation is regulated by several different stim- uli, dark-induced energy starvation results in a bZIP1/bZIP53dependent induction of Pro degradation.

\section{bZIP1 and bZIP53 Regulate the Level of Asn and the Branched-Chain Amino Acids Leu, Ile, and Val}

To determine whether bZIP1 and bZIP53 regulate amino acid metabolism in general during the dark-induced starvation response, a comprehensive amino acid analysis was performed. In wild-type plants, the total amount of amino acid increased in response to prolonged darkness. By contrast, Pro35S:bZIP53 plants showed a significantly stronger accumulation of total amino acids, whereas in the bzip1 bzip53 double mutant the increase was less pronounced (Figure 3A). During an extended night, an increase was observed especially for the levels of the branched-chain amino acids (BCAAs) Leu, Ile, and Val as well as for Asn (Figure 3B; see Supplemental Data Set 1 online). The increase in BCAA levels, in particular of Leu and lle, was strongly repressed in the Pro35S:bZIP1 and Pro35S:bZIP53 plants, indicating that the bZIP regulators promote the degradation of Leu and Ile. The Val levels followed a similar accumulation pattern; however, it was less pronounced (Figure 3B; see Supplemental Data Set 1 online). The impact of bZIP1 and bZIP53 on Asn metabolism differed considerably from that of Leu, Ile, and Val. Dark-induced Asn levels were enhanced by the overexpression of bZIP53 but not bZIP1, whereas the bzip 1 bzip53 plants displayed a slightly reduced amount of Asn (Figure 3B; see Supplemental Data Set 1 online). Together, our data suggest that bZIP1 and bZIP53 participate in the transcriptional reprogramming of amino acid metabolism during the dark-induced starvation response.

\section{bZIP1 and bZIP53 Regulate the Expression of Genes} Involved in the Dark-Induced Synthesis of Asn and BCAAs

As bZIP1 and bZIP53 are responsible for modulation of amino acid levels, we tested the expression of genes that are related to the corresponding metabolic pathways. Asn is the major source for N/C transport in darkness (Lam et al., 1994, 1998, 2003). Asn biosynthesis is derived from pyruvate and requires the coordinated, transcriptional upregulation of several genes (Lin and $\mathrm{Wu}, 2004$; Figure 4A). Expression of these genes during

Figure 2. (continued).

(C) Analysis of ProDH transcript accumulation in the wild type (black bars), Pro35S:bZIP1 (gray bars), Pro35S:bZIP53 (hatched bars), and bzip1 bzip53 (white bars) plants after short-term dark treatment as described in Figure 1A. Rosette leaves of 10 3-week-old plants were pooled and used for RNA preparation and GPCR at the time points indicated. Fold change compared with wild-type expression is depicted at $0 \mathrm{~h}$. Mean value and SD of two replicates are given. Expression analysis of $b Z I P 1$ and bZIP53 is provided in Supplemental Figure 4 online. For visualizing the differences in transcript levels, the $y$ axis is broken twice, at 1.5- and 30 -fold induction.

(D) Quantification of Pro levels of the plants described in (B) and (C) after dark treatment. Given are ng Pro/mg dry weight (DW) as mean values and SD of two independent repetitions. Asterisks represent significant differences between wild-type, overexpressor, and mutant plants at the indicated time points (two-way ANOVA; ${ }^{*} \mathrm{P}<0.05$ and ${ }^{* * *} \mathrm{P}<0.001$ ).

(E) The transcript abundance of bZIP1 and bZIP53 is regulated by sugar depletion. Three-week-old wild-type plants were cultivated in hydroponic culture as depicted in the scheme. Plants were harvested $3 \mathrm{~h}$ after the beginning of the light period (L). The remaining plants were incubated in darkness for $24 \mathrm{~h}(\mathrm{D})$. After $24 \mathrm{~h}$, the plants were transferred to medium containing equimolar (167 mM) 3-oMG, glucose, sucrose, and PEG1000 or nonsupplemented medium as control (-). During these incubations, plants were kept in the dark. RNA was isolated from the differently supplemented cultures after 1 or $2 \mathrm{~h}$, respectively. Given are RNA gel blot analyses of ProDH, bZIP1, and bZIP53 transcripts. Loading is controlled by ethidium bromide $(\mathrm{EtBr})$ staining. 


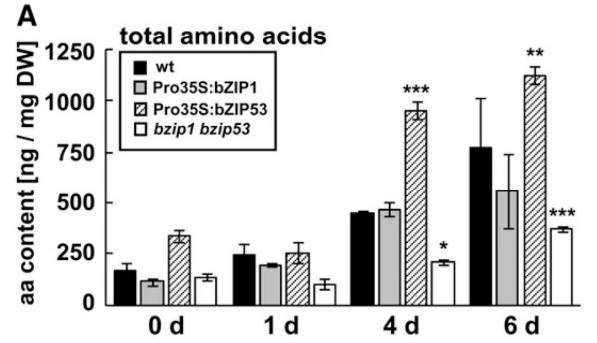

B
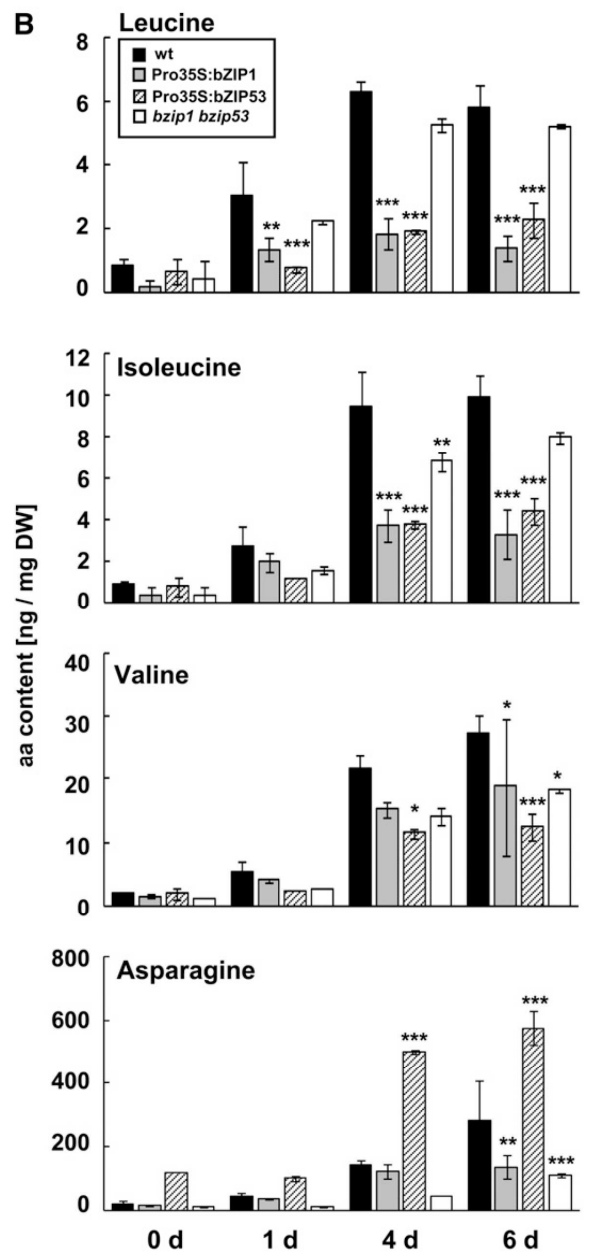

Figure 3. Quantitative Analysis of the Amount of Amino Acids in DarkTreated Plants.

The levels of total amino acid content (A) and of Leu, lle, Val, and Asn (B) Amino acid levels of the wild type (wt) (black bars), Pro35S:bZIP1 (gray bars), Pro35S:bZIP53 (hatched bars), and bzip1 bzip53 (white bars) after $0,1,4$, and $6 \mathrm{~d}$ of dark treatment are calculated as ng amino acid/mg dry weight (DW). Given are mean values and SD of two independent exper- dark-induced starvation was substantiated by public expression data (see Supplemental Figure 6A online) and confirmed by RNA gel blot analysis (Figure 4B). The tested genes encoding enzymes of the Asn biosynthetic pathway, such as GLUTAMATE DEHYDROGENASE2 (GDH2), ASPARTATE AMINOTRANSFERASE3 (ASP3), GLUTAMATE SYNTHASE (GLNS), and ASN1, were induced during $8 \mathrm{~d}$ of dark treatment (Figure 4B). The overexpression of bZIP1 resulted in an enhanced or more rapid transcript accumulation of these biosynthetic genes, whereas overexpression of bZIP53 caused constitutively high transcript levels. Accumulation of PepCK transcripts, which encode PEP CARBOXYKINASE, the first enzymatic step in Asn biosynthesis, was neither induced by darkness nor by bZIP1 overexpression. A slight accumulation of PepCK transcript was only observed when bZIP53 was overexpressed (Figure 4B).

A detailed time course of an extended night treatment was performed for the central Asn biosynthesis pathway gene ASN1. Comparable to $P r o D H$, rapid induction of $A S N 1$ transcript accumulation was detected within $4 \mathrm{~h}$ of the extended night treatment (Figure 4C). This response was strongly enhanced in Pro35S: bZIP1 plants but not in Pro35S:bZIP53 plants. These expression data seem to contradict the metabolic analysis because the Asn levels were higher in Pro35S:bZIP53 than in Pro35S:bZIP1 plants. However, in contrast with bZIP53, bZIP1 also activated an ASPARAGINASE (ANS) gene (Figure 4D), which participates in the degradation of Asn (Bruneau et al., 2006). Our observations suggest that bZIP1 and bZIP53 have partly overlapping but also distinct functions in the regulation of Asn metabolism.

Ectopic expression of $b Z I P 1$ and $b Z I P 53$ leads to reduced levels of BCAA, indicating their involvement in the corresponding catabolic pathway. The mitochondrial BCAA TRANSAMINASE1 (BCAT1) gene was proposed to encode the central catabolic enzyme (Diebold et al., 2002; Schuster and Binder, 2005; see Supplemental Figures $6 \mathrm{~A}$ and $6 \mathrm{~B}$ online). However, the analysis of the six Arabidopsis BCAT genes revealed that BCAT2 and to a minor extent $B C A T 1$ were induced during dark treatment. As depicted in Supplemental Figure 6 online, bZIP1 strongly enhances BCAT2 transcript accumulation in the dark. The BCAT2 enzyme is localized in the chloroplasts, where it contributes to Leu and Glu biosynthesis (Schuster and Binder, 2005). Therefore, the plastidic deamination reaction of BCAT2 in the dark might supply the cell with Glu, which in turn is essential for Asn biosynthesis (see Supplemental Figure 6B online). In conclusion, the dark-induced accumulation of BCAT2 transcript is rather linked to the dark-induced Asn biosynthesis than to darkinduced BCAA degradation.

Promoters of Amino Acid Metabolic Genes Are Regulated by bZIP1 and bZIP53 in Response to Energy Starvation in Protoplasts

To assess the direct impact of bZIP1 and bZIP53 on gene regulation, the activity of $A S N 1$ and $P r o D H$ promoter:reporter

iments. Asterisks represent significant differences between wild-type, overexpressor, and mutant plants at the indicated time points (two-way ANOVA; ${ }^{\star} P<0.05,{ }^{\star \star} P<0.01$, and $\left.{ }^{\star \star \star} P<0.001\right)$. 
A

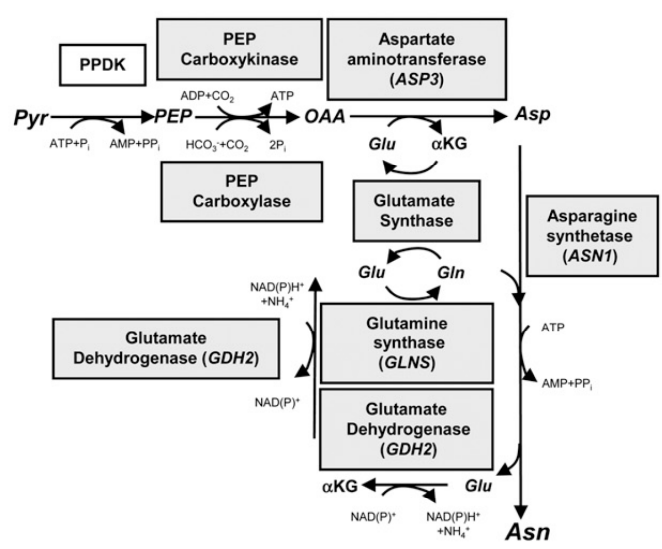

C
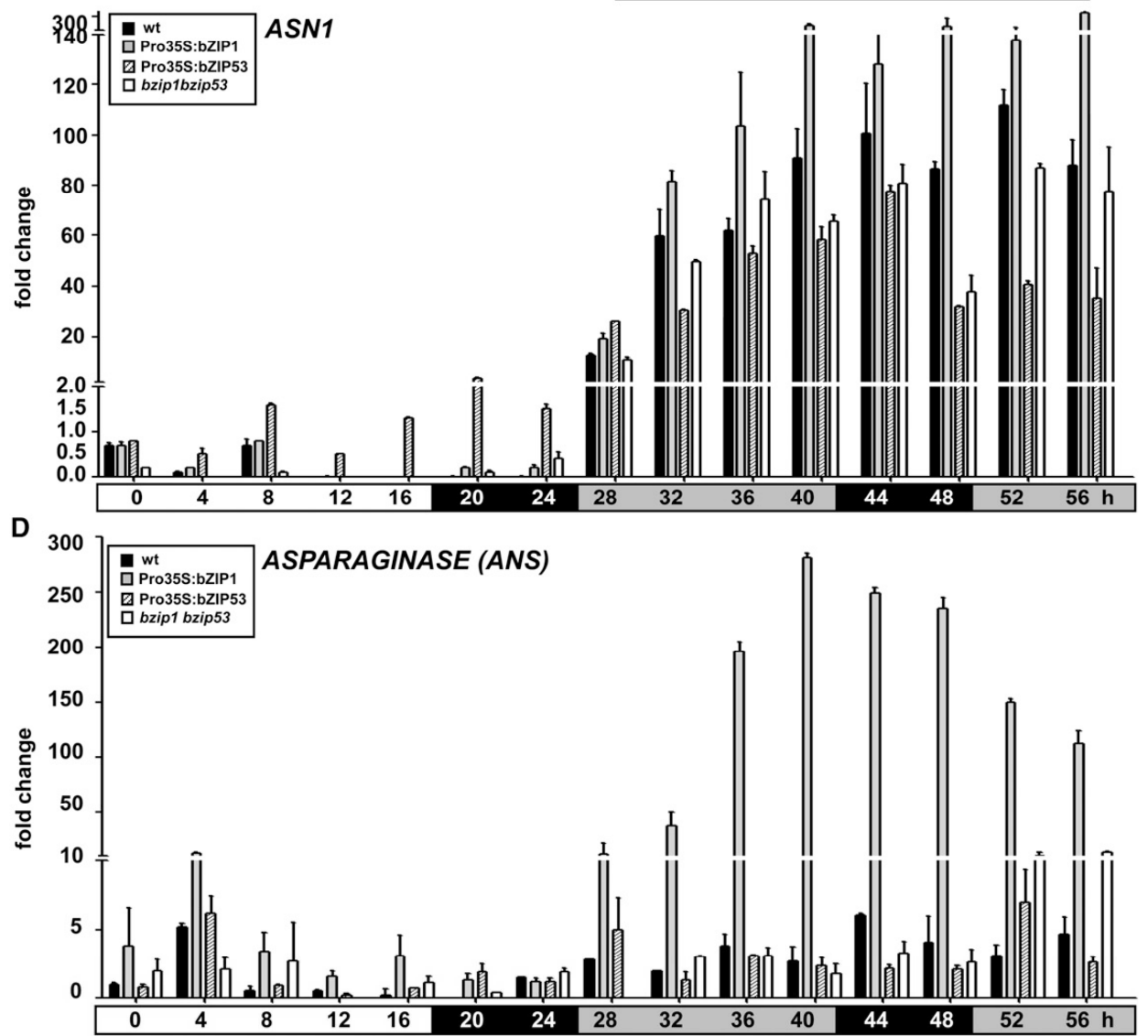

Figure 4. bZIP1 and bZIP53 Regulate Gene Expression of Asn Metabolism during Extended Night Treatment. 
constructs (ProASN1:GUS and ProProDH:GUS) was studied in transiently transfected protoplasts. Starvation was induced by either the transfer of light-cultivated protoplasts to darkness or treatment of light-cultivated protoplasts with the photosystem II inhibitor DCMU. Both starvation treatments induced the transcriptional activity of the ProASN1:GUS and ProProDH:GUS reporter genes, demonstrating that the protoplast system can be used to analyze starvation-induced transcription (see Supplemental Figure 7 online). bZIP53 upregulated the activity of the ProDH promoter both after light and dark cultivation. In contrast with the results obtained in transgenic plants, expression of bZIP1 in protoplasts did not induce both reporter constructs, indicating that additional factors are needed to fulfill its function in plants, which are not present in protoplasts. The differences observed in bZIP1 and bZIP53 function in protoplasts were not due to different protein levels as confirmed by immunoblot analysis (see Supplemental Figure $7 \mathrm{C}$ online).

\section{bZIP1 and bZIP53 Directly Regulate ASN1 and ProDH Promoter Activity via G-Boxes or ACT cis-Elements in the Starvation Response}

Using the protoplast transfection system, we analyzed whether the starvation response is mediated by ACGT motifs, which represent typical binding sites for bZIP TFs (Jakoby et al., 2002). As summarized in Supplemental Table 1 online, all promoters of the Asn biosynthesis genes and of $P r O D H$ and $B C A T 2$ harbor at least one ACGT motif. In the ASN1 promoter, two G-boxes (CACGTG) were found, and G-box 1 was identified as the crucial cis-element in mediating SnRK1 responses (Baena-González et al., 2007). Sequential mutation in the ProASN1:GUS reporter gene demonstrated that the dark-induced transcription and the bZIP1/bZIP53-mediated enhancement of transcription depended exclusively on G-box 1 (Figure $5 \mathrm{~A}$ ). No alteration in the ProASN1:GUS reporter gene activity was observed with a lossof-function mutation in G-box 2, indicating that the position of the hexameric CACGTG sequence within the promoter is important to mediate the starvation-related gene expression in protoplasts.

To define whether the identified genes involved in amino acid metabolism are direct targets of the bZIP factors, chromatin immunoprecipitation (ChIP) experiments were performed with transgenic Arabidopsis lines expressing the HA-tagged version of bZIP1. Using primers that amplify the G-box 1/2 promoter region, we could show direct binding of $\mathrm{HA}-\mathrm{bZIP1}$ proteins to the ASN1 promoter (Figure 5B).
Previous results revealed pronounced differences in the regulation of $P r o D H$ and $A S N 1$. The ProDH promoter harbors no G-box, but a C-box (GACGTC) and two ACT elements (ACTCAT), which are proposed to be bZIP binding sites involved in ProDH regulation (Satoh et al., 2004; see Supplemental Table 1 online). Whereas single mutations in the ACTCAT elements (Figure 5 C) or C-box (see Supplemental Figure 8 online) resulted in minor but significant effects on dark-induced $\mathrm{ProDH}$ activation, multiple mutations in two cis-elements completely abolished inducibility of the ProDH promoter. From these data, we propose a crucial combinatorial in vivo function of these elements in the darkinduced $\mathrm{ProDH}$ activation.

Recently published ChIP experiments demonstrated the in vivo binding of bZIP53 to the ProDH promoter (Weltmeier et al., 2006). In addition, ChIP analyses using primers surrounding the ACT elements and Pro35S:HA-bZIP1 plants also revealed a direct binding of bZIP1 to the ProDH promoter (Figure 5D). Immunoblot analysis of chromatin derived from light- and dark-grown plants showed equal amounts of HA-tagged bZIP1 protein in the ChIP assays (Figure 5E). Therefore, the binding activity of bZIP1 to the ProDH promoter was independent of the light/dark regime.

\section{Multiple bZIP Mutants and Plants Expressing EAR}

Repressor Fusions of bZIP Factors Are Partially Impaired in Dark-Induced Transcription of Amino Acid Metabolic Genes

The bzip 1 bzip53 double mutant showed only limited impairment in dark-induced ProDH and ASN1 transcript accumulation (Figures $2 \mathrm{C}$ and $4 \mathrm{C}$; see Supplemental Figure 5 online). Therefore, we applied an alternative loss-of-function approach. Fusions between bZIP53 and bZIP1 and the EAR repressor domain (Hiratsu et al., 2003) were generated and tested for their impact on the $\mathrm{ProDH}$ reporter in protoplasts. As shown in Figure $6 \mathrm{~A}$, the light- and dark-induced activation of the ProProDH:GUS reporter was completely abolished by EAR-bZIP1 and strongly reduced by EAR-bZIP53. Expression of the fusion proteins was confirmed by immunoblot analysis as demonstrated in Supplemental Figure 9A online. These data further substantiate our hypothesis that bZIP1 and bZIP53 play a crucial role in the regulation of darkinduced ProDH transcription. However, due to their heterodimerization properties, other bZIPs, presumably members of the $\mathrm{C}$ / S1 network, are likely candidates for mediating the dark-induced starvation response (Ehlert et al., 2006). We therefore included quadruple T-DNA mutants of bZIP1 and bZIP53 with different group C bZIPs (bzip1 bzip53 bzip9 bzip63 and bzip1 bzip53

Figure 4. (continued)

(A) The Asn biosynthesis pathway according to Lin and Wu (2004). $\alpha$ KG, $\alpha$-keto-glutarate; Pyr, pyruvate; PEP, phosphoenolpyruvate; PPDK, pyruvate orthophosphate dikinase.

(B) RNA gel blot analysis of the indicated genes corresponding to the enzymatic steps depicted in the Asn biosynthesis pathway in (A) after long-term dark treatment for 0 to $8 \mathrm{~d}$. Compared are wild-type (wt), Pro35S:bZIP1, and Pro35S:bZIP53 plants. During dark induction, plants were harvested at the indicated days at 5 PM. As a loading control, ethidium bromide stainings are provided for each hybridization experiment.

(C) and (D) Induction of ASN1 (C) or ANS (D) after short-term dark treatment. Rosette leaves of 10 3-week-old plants were pooled and used for RNA preparation and GPCR at the time points indicated. Fold change compared with wild-type expression is depicted at $0 \mathrm{~h}$. Mean value and SD of two replicates are given. The wild type (black bars), Pro35S:bZIP1 (gray bars), Pro35S:bZIP53 (hatched bars), and bzip1 bzip53 (white bars) analyzed by qPCR as described in Figure 1A. For visualizing the differences in transcript levels of ASN1, the $y$ axis is broken twice, at two- and 140-fold induction. The $y$ axis of the ANS plot is broken once at 10 -fold induction. 

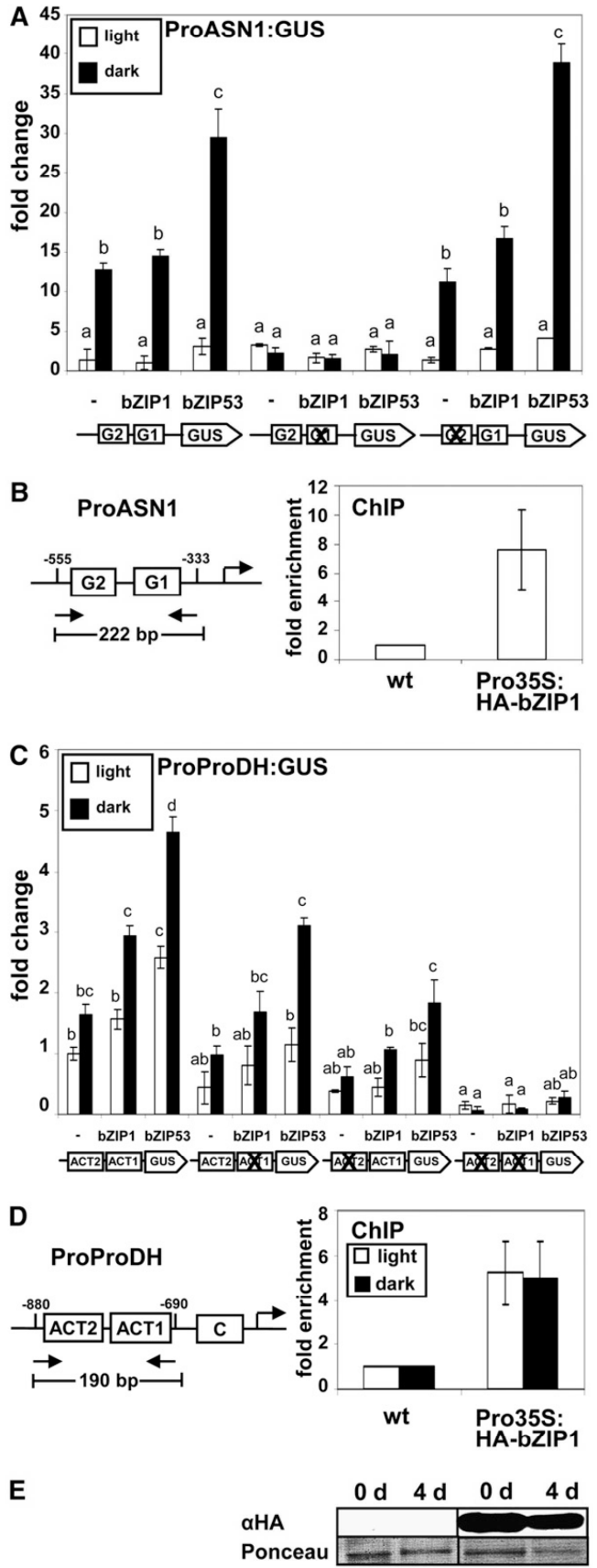

Figure 5. bZIP1 Binds Directly to the ASN1 and ProDH Promoters and Mediates Starvation Responses via G-Box (CACGTG) or ACTCAT cisElements. bzip10 bzip25) in our study. As demonstrated in Supplemental Figures 9B and 9C online, depending on the particular bZIP gene, complete null alleles or knockdown alleles were obtained in the respective mutant lines. The accumulation of $A S N 1, P r o D H$, and $B C A T 2$ (Figure 6B) transcripts was considerably impaired during extended dark treatment in the quadruple mutants, although no complete loss of transcript accumulation was observed. Surprisingly, after long-term dark treatment, gene expression was partially restored, indicating that the plant harbors regulatory mechanisms to substitute for the loss of particular bZIP proteins.

\section{DISCUSSION}

In this work, we identified two bZIP TFs, namely, bZIP1 and bZIP53, that translate low energy signals into an altered transcriptional pattern of amino acid metabolic genes in Arabidopsis. As outlined in the model in Figure 7, starvation activates in particular bZIP1 transcriptionally and posttranscriptionally, the latter by a conserved system of uORFs (Wiese et al., 2004; Weltmeier et al., 2009). Presumably by heterodimerization with other members of the C/S1 bZIP TF network, bZIP1 and bZIP53 initiate the change in transcriptional activity by binding to ACGT or ACTCAT-like cis-elements within the promoters of metabolic target genes. In conclusion, bZIP1 and bZIP53 are proposed to

(A) Arabidopsis protoplasts were transformed with a ProASN1:GUS reporter construct or the indicated promoter mutations (see diagrams beneath the $x$ axis). After cotransformation with the effector plasmids (Pro35S:bZIP1 or Pro35S:bZIP53), reporter induction was compared in constant darkness (black bars) or in constant light (white bars) conditions. Given is the fold change with respect to the empty vector control experiment without any bZIP construct added $(-)$ under constant light. Four transfection experiments were used to calculate mean values and SD. Significance was tested by one-way ANOVA analysis following Tukey's post-test $(P<0.05)$

(B) Direct binding of bZIP1 to the ASN1 promoter as demonstrated by ChIP. ASN1 promoter structure and primer binding sites are indicated on the left. Chromatin extracts from wild-type (wt) plants and Pro35S:HAbZIP1 were subjected to GPCR analysis with ASN1 promoter-specific primers after immunoprecipitation with an anti-HA antibody $(\alpha-\mathrm{HA})$. Ct values for Pro35S:HA-bZIP1 samples were subtracted from the Ct values of the equivalent wild type. For normalization, an actin (ACT7) gene was used. Calculated are induction levels with respect to the wild-type samples. Given are mean values and SD of three independent experiments.

(C) Analysis of the ProProDH:GUS constructs as described in (A). Additional promoter analyses are provided in Supplemental Figure 8 online.

(D) ChIP experiment of wild-type and Pro35S:HA-bZIP1 plants as described in (B). Chromatin was isolated from 3-week-old plants grown under normal light/dark cycle (white bars) or plants cultivated in an extended night for $4 \mathrm{~d}$ (black bars). Given are mean values and SD of three repetitions.

(E) Immunoblot analysis of chromatin derived from wild-type and Pro35S:HA-bZIP1 plants detected with an $\alpha \mathrm{HA}$ antibody indicates a comparable HA-bZIP1 protein abundance in light- and dark-treated plants. As a loading control, Ponceau staining of the protein preparation is given (bottom panel). 

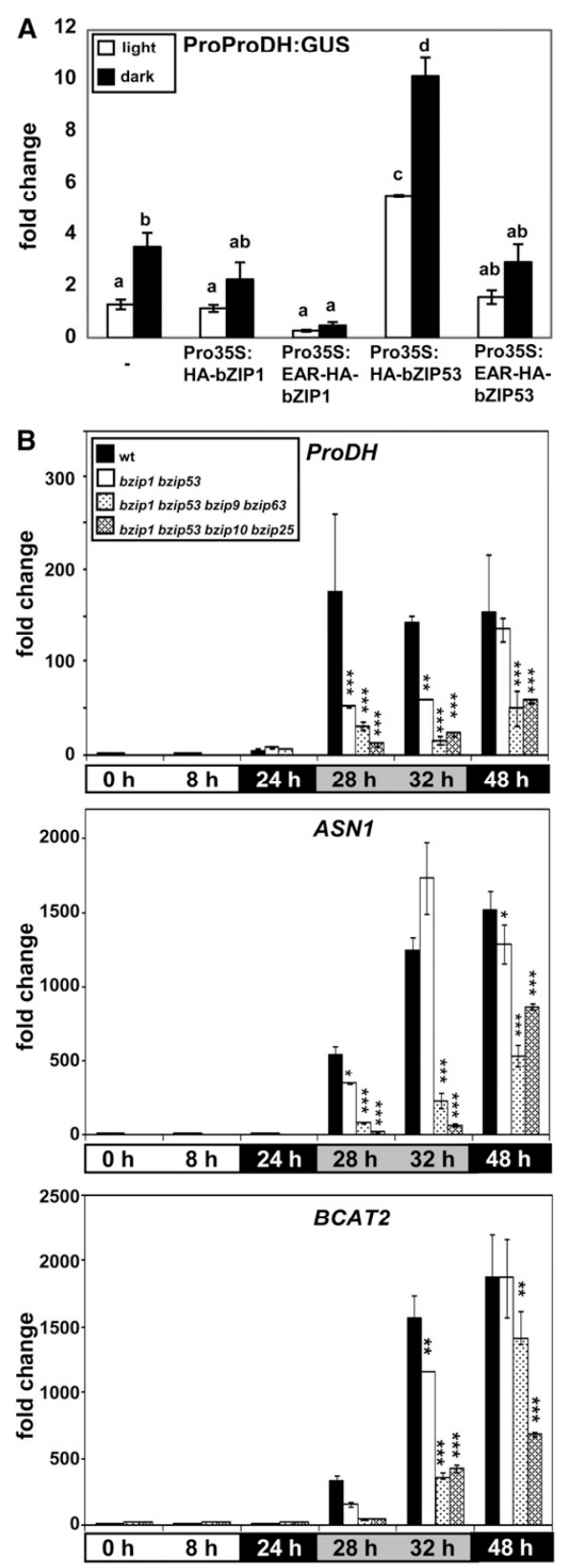

Figure 6. Impact of bZIP Factors on Target Gene Expression Using bZIP-Specific Loss-of-Function Approaches.

(A) EAR repressor fusions of bZIP1 and bZIP53 reveal a regulatory function in dark-induced ProDH transcription. Arabidopsis protoplasts were transiently transformed with a ProProDH:GUS reporter and cOtransfected with Pro35S-driven reporters (HA-bZIP1, HA-bZIP53, HA- mediate transcriptional metabolic reprogramming in response to starvation.

The bZIP TFs bZIP1 and bZIP53 Are Regulated by Energy Deprivation, Both at the Transcriptional and Posttranscriptional Level

In this work, bZIP1, and to a minor extent also bZIP53, was found to be transcriptionally upregulated by conditions that lead to energy deprivation. Feeding experiments with sucrose and glucose, but not 3-oMG, repress bZIP1 and bZIP53 transcription (Figure 2E). 3-oMG is taken up by the cells but is not metabolized and appears not to signal via the hexokinase-dependent sugar signaling pathway (Cortès et al., 2003). These data indicate that sugar signaling regulates the transcription of $b Z I P 1$ and $b Z I P 53$, supporting recent findings by Kang et al. (2010). Since long-time dark treatments, which have frequently been applied for darkinduced senescence studies (Gan, 2003; Lin and Wu, 2004; Buchanan-Wollaston et al., 2005), do not reflect natural environmental conditions, we performed short-term experiments that described the detailed expression changes after extended night treatments and defined bZIP1 and bZIP53 as putative transcriptional regulators in the starvation response (Figure 1A).

For assaying starvation responses, plant and protoplast systems were applied using culture conditions in the dark or incubation with the photosystem II inhibitor DCMU (e.g., Figure 1A; see Supplemental Figures 7A and 7B online). Recently, other stresses, such as anaerobic conditions, have been found equally useful (Baena-González et al., 2007). Altogether these treatments let to comparable responses with respect to transcription of starvation-induced genes. Environmental stresses, which also lead to a low energy status of the cell, can be assumed to interfere with primary metabolism and therefore might also input into the starvation signaling network (Baena-González and Sheen, 2008).

Recent bioinformatic network analyses suggest that $b Z I P 1$ is regulated by the circadian clock (Gutiérrez et al., 2008). Interestingly, CIRCADIAN CLOCK ASSOCIATED1, which is an integral component of the Arabidopsis clock (Yakir et al., 2007), was shown to directly bind to the bZIP1 promoter (Gutiérrez et al., 2008). Although a detailed molecular analysis is still elusive, it is

EAR-bZIP1, and HA-EAR-bZIP53). Induction by cultivation in constant dark (black hars) conditions is compared with expression in constant light (white bars). Depicted is the fold change compared with the promoter in the light. Four transfection experiments were used to calculate mean values and SD. Different letters indicate significant differences, tested by one-way ANOVA analysis following Tukey's post-test $(P<0.05)$. Expression of the effector constructs was confirmed by immunoblot analysis (see Supplemental Figure 9A online). (B) GPCR analysis of ProDH, ASN1, and BCAT2 after extended night treatment as described in Figure 1. Rosette leaves of 103 -week-old plants were pooled and used for RNA preparation and GPCR at the indicated time points. Fold change compared with the wild type (wt) is indicated at $0 \mathrm{~h}$. Mean value and SD of two replicates are given. Asterisks represent significant differences between wild-type and mutant plants at the indicated time points (two-way ANOVA; ${ }^{*} P<0.05$, ${ }^{* *} P<0.01$, and ${ }^{\star \star \star} P<0.001$ ). Impaired expression of the corresponding bZIP genes is demonstrated in Supplemental Figures 9B and 9C online. 


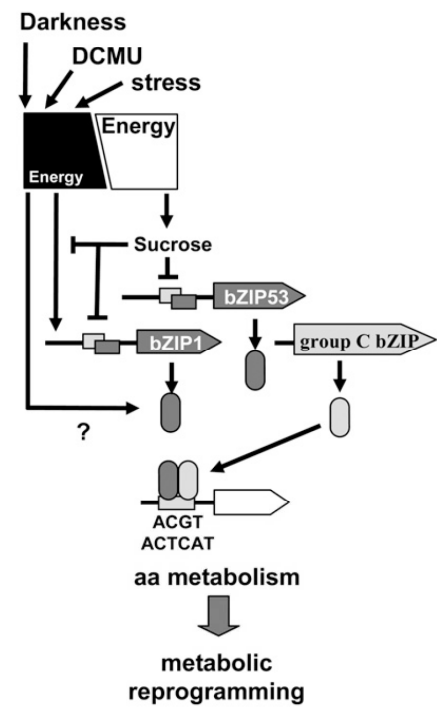

Figure 7. Model Summarizing the Function of bZIP Factors in the Energy Deprivation Response.

Starvation activates bZIP1 and bZIP53 transcriptionally and posttranscriptionally, the latter by a conserved system of uORFs. Presumably by heterodimerization with other members of the C/S1 bZIP network, bZIP1 and bZIP53 initiate a change in transcriptional activity by binding to ACGT or ACTCAT-like cis-elements within the promoters of metabolic target genes, causing a reprogramming of primary metabolism in response to low energy stress.

tempting to speculate that a regulatory energy management network exists in plant cells, which integrates $\mathrm{C}$ and $\mathrm{N}$ availability and clock-initiated day/night rhythms to regulate bZIP1-dependent gene expression.

In addition to transcriptional regulation, further posttranscriptional mechanisms might account for bZIP1 and bZIP53 regulation. For all group S1 bZIPs, a posttranscriptional repression by sucrose (sucrose-induced repression of translation) was demonstrated (Weltmeier et al., 2009). With respect to bZIP1 and bZIP53, these mechanisms result in an additive effect, leading to high intracellular levels of the bZIP TFs during energy deprivation (Figure 7). Thus, bZIP1 and bZIP53 are candidates to execute a crucial function in metabolic reprogramming during the starvation response.

\section{bZIP1 Is Regulated Posttranslationally by a} Starvation-Derived Signal

Although bZIP1 is strongly upregulated by transcriptional and posttranscriptional mechanisms, its function in gene regulation depends on an additional signal, which is initiated by dark induced energy deprivation. Importantly, this observation resulting from studies in transgenic plants was not detectable in the protoplast system: Arabidopsis bZIP1 overexpression leads only to a minor change in target gene activation. Apparently, an unknown component is missing in the protoplast system. Therefore, these findings emphasize the importance of studying the whole plant system

bZIP53 is predominantly regulated on the level of protein amount. When overexpressed, it activates transcription independently of the energy status of the cell, whereas bZIP1 activity is further fine-tuned at the posttranslational level by a starvationdependent signal. Recently, KIN10 and KIN11, two kinases of the SnRK1 family, have been demonstrated to orchestrate starvation responses, in particular on the transcriptional level (BaenaGonzález et al., 2007; Baena-González and Sheen, 2008). It remains to be analyzed whether the starvation signal regulating bZIP1 function is directly or indirectly mediated by these kinases.

\section{bZIP1 and bZIP53 Show Overlapping but Distinct Functions} in the Low Energy Response

In this work, ectopic overexpression of bZIP1 results in an early senescence phenotype in the dark, which is characterized by rapid loss of chlorophyll (Figures 1C and 1D). As defined by marker gene expression, this phenotype reflects a dark-induced starvation response, but not the natural senescence (see Supplemental Figure 3 online; van der Graaff et al., 2006). This early senescence phenotype is less pronounced in Pro35S:bZIP53 plants. Furthermore, distinct differences in the function of bZIP1 and bZIP53 become obvious when these plants were studied under a normal day/night cycle. As confirmed by immunoblot analysis, high-level expression of bZIP1 did not lead to obvious phenotypic changes, whereas medium-level expression of bZIP53 resulted in significantly reduced plant growth (Alonso et al., 2009). These findings indicate pronounced differences in bZIP1 and bZIP53 function.

Overexpression of bZIP1 and bZIP53 in transgenic plants revealed that both TFs are capable of regulating the amino acid metabolic genes proposed to be upregulated during the darkinduced starvation response, such as genes involved in Asn biosynthesis and Pro and BCAA metabolism (e.g., Figures 2C, 4B, and 4C; see Supplemental Figure 6C online). ASN1, the key gene of the Asn biosynthesis pathway, is characterized by a complex transcriptional regulation (Lam et al., 1998, 2003). Transcription of the Asn pathway genes, such as $A S P 3, G D H 2$, and $A S N 1$, appears to be coregulated and is induced after dark treatment but repressed when sugar is available (Lin and $\mathrm{Wu}$, 2004). In the light, ectopic expression of bZIP53 leads to constitutive activation of $P r o D H, A S P 3, G D H 2$, and ASN1, whereas bZIP1 provokes only minor effects.

Changes in the transcriptional levels of amino acid metabolic genes are well reflected on the amino acid level. For instance, the amount of Pro is significantly reduced in Pro35S:bZIP1 and Pro35S:bZIP53 plants (Figure 2D). Contrarily, the amount of Asn is induced exclusively in Pro35S:bZIP53 plants (Figure 3B). As demonstrated on the transcriptional level, bZIP1 but not bZIP53 enhances expression of an ANS (Figure 4D), which leads to the degradation of Asn (Bruneau et al., 2006). Again, bZIP53 and bZIP1 show distinct differences in target gene selection. This might explain the observed differences in the metabolite profiles. 
Ectopic expression of bZIP1 and bZIP53 leads to reduced levels of BCAAs (Figure $3 \mathrm{~B}$ ), indicating the involvement of both TFs in a BCAA degradation pathway. BCAT2 transcription follows the pattern of the other analyzed, dark-induced amino acid metabolic genes, and bZIP1 and bZIP53 enhance BCAT2 transcription (see Supplemental Figure $6 \mathrm{C}$ online). However, it is not clear whether the BCAT2 enzyme is involved in an anabolic or catabolic context. In the dark, chloroplastic proteins, such as ribulose-1,5-bisphosphate carboxylase/oxygenase, are degraded to provide amino acid to the starved nitrogen metabolism. It was speculated that the deamination reaction of BCAT2 in the dark supplies the cell with Glu, which is essential for Asn biosynthesis (Schuster and Binder, 2005). Hence, dark-induced BCAT2 expression as well as the BCAA degradation might be closely linked to the dark-induced Asn biosynthesis and consequently appear to be regulated in a coordinated fashion by the same set of bZIP TFs.

Differences in function have already been described for bZIP53 and bZIP1 (Weltmeier et al., 2009). For instance, heterodimers that include bZIP53 regulate the expression of seed maturation genes involved in desiccation tolerance, storage compound synthesis, and source-sink regulation, such as ASN1 (Alonso et al., 2009). Although bZIP1 heterodimers share the capacity to activate seed maturation genes in protoplasts, bZIP1 appears not to be involved in the regulation of these genes during seed maturation (Weltmeier et al., 2009). Thus, bZIP53 and bZIP1 have partially overlapping but distinct functions that are probably defined by expression patterns and/or posttranslational mechanisms.

Transcriptional Control of Amino Acid Metabolic Genes by bZIP1 and bZIP53 Is Mediated by Binding to G-Box, C-Box, or ACTCAT cis-Elements

As demonstrated in transiently transfected protoplasts, one of the two G-boxes (G-box 1) in the promoter of ASN1 is essential for the dark activation of this gene (Figure $5 \mathrm{~A}$ ). Our promoter deletion experiments further substantiate the hypothesis that bZIP1 and bZIP53 signal via the G-box 1 in the ASN1 promoter. G-box-like cis-elements, which are characterized by their ACGT core, are typical binding sites for bZIP TFs as demonstrated for bZIP53 by in vitro and in vivo binding assays (Alonso et al., 2009). We used HA-tagged bZIP1 in ChIP experiments to confirm its direct binding to $P r O D H$ and $A S N 1$ promoter regions (Figures 5B and 5D). Although, due to overexpression, the ChIP data have to be interpreted with care, they are supported by results of the protoplast assays. Our combined data suggest that the analyzed ProDH and ASN1 promoters are direct in vivo targets of bZIP1. Dark-induced recruitment of bZIP1 to its target promoters is one possible regulatory mechanism that would explain stimulusinduced target gene activation. However, dark-induced enhancement of bZIP1 DNA binding to the ProDH promoter was not detected in the ChIP experiments, at least at the time points used in this study. Surprisingly enough, the ProDH promoter harbors no G-box but a closely related, ACGT core-containing C-box and two ACTCAT motifs. The ACTCAT motifs were shown to be bound by group S1 bZIPs and are involved in the hypoosmolarity response (Satoh et al., 2002, 2004; Weltmeier et al., 2006).
Multiple mutations in ACTCAT and C-box elements confirm a crucial and additive impact of all these cis-elements on basic and inducible $\mathrm{ProDH}$ promoter activity. These data suggest that differences in ASN1 and ProDH expression patterns are caused by the combination and location of the identified cis-elements in their promoter structures. Furthermore, bZIP heterodimerization might alter target site recognition; therefore, it is tempting to speculate that different promoters will recruit particular sets of bZIP heterodimers.

\section{Redundant bZIP Factors Can Partially Substitute for Loss of bZIP1 and bZIP53}

Expression of bZIP1 and bZIP53 fusion proteins containing a C-terminal EAR repressor domain (Hiratsu et al., 2003) completely abolishes or significantly reduces dark-induced ProDH expression in protoplasts (Figure 6A). Since the repressor-modified TF blocks specific promoter binding sites when overexpressed, this method was applied to compete with redundantly active TFs to interfere with their function. As single bzip1 and bzip53 T-DNA mutants and even a bzip 1 bzip53 double mutant do not lead to dramatic impairment of amino acid target gene expression (see Supplemental Figure 5 online), the protoplast data support the view that a functional redundant bZIP TF network is operating in the starvation response. bZIP2 expression patterns are slightly similar to the one observed for bZIP53. However, since bZIP2 T-DNA insertion lines are not available and $b Z I P 2$ overexpression results in severely dwarfed, sterile plants, bZIP2 could not be included in the experimental setup. Strong interference with normal plant growth was also observed by overexpression of bZIP11 (Hanson et al., 2008). Inducible expression combined with transcriptome analysis was used to identify $\mathrm{ProDH}$ and ASN1 as bZIP11-regulated genes. However, although bZIP11 has the capacity to regulate these metabolic genes, it is probably not involved in the starvation response as its expression is upregulated by sugar and downregulated by darkness. As depicted in Supplemental Figure 1 online, several bZIP genes, such as bZIP41 or bZIP54, show appropriate expression patterns to fulfill a function in the energy deprivation response. Also, the group C factors bZIP63, bZIP9, and bZIP25, which form heterodimers with group S1, might be candidates. Specific bZIP heterodimerization has been shown to be important for bZIP53 activity and function (Ehlert et al., 2006; Weltmeier et al., 2006). Consequently, quadruple C/S1 T-DNA insertion mutants show strongly impaired dark-induced target gene expression (Figure $6 \mathrm{~B}$; see Supplemental Figure 9D online). Surprisingly, this effect is only transient and the plant can partially compensate for the loss of bZIP gene activity during long-term dark adaptation. Overlapping functional redundancy appears to be a frequently observed feature in particular in signaling networks. The regulation of target genes by C/S1 bZIPs is complex; however, it is more stable with respect to mutations and more flexible in terms of its potential to fine tune regulation. Although the plant benefits from this flexibility, the regulatory circuits controlling this TF network remain elusive.

Knowledge of the transcriptional regulators is crucial for understanding the plant energy control system and is a first step to 
establishing biotech approaches to increase yield and stress tolerance of crop plants.

\section{METHODS}

\section{Plant Cultivation and Treatment}

For plant and protoplast transformation, amino acid measurement, qPCR, RNA gel blot, and ChIP experiments, Arabidopsis thaliana ecotype Columbia (Col-0) was grown on soil under long-day conditions of $16-\mathrm{h}-$ light/8-h-dark cycles. Dark treatment was performed for $4 \mathrm{~h}$ to $8 \mathrm{~d}$ using 3-week-old soil-grown plants. For RNA gel blot analysis, the first harvesting time point $(0 \mathrm{~d})$ was at $5 \mathrm{PM}$, during the light period. Material for qPCR was harvested as indicated, starting at the beginning of the light period (8 AM). For hydroponic culture, the procedure described by Gibeaut et al. (1997) has been modified. Three-week-old plants were grown on a mesh support under short-day conditions. The media were supplemented with sugars according to the description in Figure 2E. Floral dip transformations were performed using the Agrobacterium tumefaciens strain GVG3101 (Weigel and Glazebrook, 2002). Transgenic plants and T-DNA insertion lines are summarized in Supplemental Table 2 online. Homozygous mutants were identified by PCR as described (http://signal.salk. edu/tdnaprimers.2.html) using the primers described in Supplemental Table 3 online.

\section{Molecular Biology and Physiology Methods}

RNA gel blot analysis and histochemical GUS staining were described in Weltmeier et al. $(2006,2009)$. Hybridization probes were PCR generated using the primers summarized in Supplemental Table 3. RNA gel blot analysis was repeated three times with comparable results. Immunoblot analysis was performed according to Weltmeier et al. (2006) using $\alpha \mathrm{HA}$ antisera (Santa Cruz Biotechnology). Aa analysis was performed as described in Pilot et al. (2004).

\section{qPCR}

RNA was isolated from pooled material of the rosettes of 103 -week-old plants. CDNA synthesis and qPCR analysis were performed as described by Fode and Gatz (2009). Cycling conditions were as follows: $10 \mathrm{~min}$ at $95^{\circ} \mathrm{C}, 40$ cycles of 20 s at $95^{\circ} \mathrm{C}, 10$ s at $55^{\circ} \mathrm{C}$, and 30 s at $72^{\circ} \mathrm{C}$, followed by a default dissociation stage program to detect nonspecific amplification. Amplification products were visualized by SYBR green. The ubiquitin (UBI5) gene was used for sample normalization. Other gene-specific oligonucleotides are described in Supplemental Table 3 online. Statistical analysis was performed with the GraphPad Prism or OriginPro 8.1 software using the tests indicated in the figure legend.

\section{Transient Expression Assays and Constructs}

Protoplast isolation, transformation, construction of effector plasmids, and immunoblot analysis were performed according to Ehlert et al. (2006). ProProDH:GUS and ProASN1:GUS reporter constructs were obtained by inserting the PCR-amplified promoters into the PBT10-TATA-GUS vector (Sprenger-Haussels and Weisshaar, 2000) using Pstl/Ncol or Xbal/Ncol restriction sites, respectively. The PCR primers are given in Supplemental Table 3 online. Mutation of promoter cis-elements was performed using the Quick Change site-directed mutagenesis kit (Stratagene). For reporter gene assays, $5 \mu \mathrm{g}$ reporter and $14 \mu \mathrm{g}$ of effector were used, unless stated otherwise. One microgram of Pro35S:NAN plasmid was added for normalization (Kirby and Kavanagh, 2002). Protoplasts were incubated overnight for $16 \mathrm{~h}$ in constant light or constant darkness. For treatment with DCMU, $20 \mu \mathrm{M}$ DCMU was added to the solution for incubation overnight. Four transfection experiments were used to calculate mean values and standard deviations of relative GUS/NAN activities as described by Ehlert et al. (2006).

\section{ChIP}

ChIP was performed as described by Weltmeier et al. (2006) and Alonso et al. (2009) using an HA-specific antibody (Abcam). Primers are given in Supplemental Table 3 online. The difference between the resulting $C(t)$ values of wild-type and Pro35S:HA-bZIP1 overexpressor was calculated and normalized with the input controls of these samples, which were analyzed with the same primers. For further normalization, PCR was performed with unspecific actin (ACT7) promoter primers (see Supplemental Table 3 online).

\section{Accession Numbers}

Arabidopsis Genome Initiative identifiers for the genes mentioned in this article are as follows: bZIP53 (At3g62420), bZIP1 (At5g49450), bZIP63 (At5g28770), bZIP10 (At4g02640), bZIP25 (At3g54620), bZIP9 (At5g24800), ProDH (At3g30775), ASN1 (At3g47340), GDH2 (At5g07440), ASP3 (At5g11520), GLNS (At5g37600), PepCK (At5g65690), ANS (At3g16150), CAB (At1g29920), SAG103 (At1g10140), YLS3 (At2g44290), BCAT2 (At1g10070), LEA76 (At3g15670), UBI5 (At3g62250), and ACT7 (At5g09810).

\section{Supplemental Data}

The following materials are available in the online version of this article. Supplemental Figure 1. Expression of bZIP Genes during Energy Starvation.

Supplemental Figure 2. Molecular Characterization of Transgenic Plants Altering the Amount of bZIP1.

Supplemental Figure 3. Expression of Senescence Marker Genes Has Been Analyzed in Plants That Have Been Cultivated under Extended Night Treatment.

Supplemental Figure 4. Expression of bZIP1 and bZIP53 after Extended Night Treatment.

Supplemental Figure 5. Expression Analysis of Genes Involved in Amino Acid Metabolism Analyzed in Single and Double T-DNA Insertion Mutants of bZIP1 and bZIP53.

Supplemental Figure 6. Regulation of Transcription in BranchedChain Amino Acid (BCAA) Metabolism during Dark Treatment.

Supplemental Figure 7. Analysis of Energy Deprivation Induced Transcription in Protoplasts.

Supplemental Figure 8. Mutation in the C-Box Effects ProDH Promoter Activity.

Supplemental Figure 9. Immunoblot Analysis of the EAR Repressor Approach and Characterization of Multiple T-DNA Insertion Mutants Used in This Study.

Supplemental Table 1. Summary of Putative bZIP Binding Motifs (ACGT-Like Elements) Found.

Supplemental Table 2. Summary of the T-DNA Insertion Lines Used in This Study.

Supplemental Table 3. Summary of the Oligonucleotide Primers Used in This Study.

Supplemental Data Set 1. Summary of Amino Acid Analysis of 3-Week-Old Arabidopsis Rosettes. 


\section{ACKNOWLEDGMENTS}

We thank Jennifer Krüger, Anna Herman, and Bettina Stadelhofer for their valuable technical assistance and Johannes Hanson for critical reading of the manuscript.

Received March 17, 2010; revised December 21, 2010; accepted January 12, 2011; published January 28, 2011

\section{REFERENCES}

Alonso, R., Oñate-Sánchez, L., Weltmeier, F., Ehlert, A., Diaz, I., Dietrich, K., Vicente-Carbajosa, J., and Dröge-Laser, W. (2009). A pivotal role of the basic leucine zipper transcription factor bZIP53 in the regulation of Arabidopsis seed maturation gene expression based on heterodimerization and protein complex formation. Plant Cell 21: 1747-1761.

Baena-González, E., Rolland, F., Thevelein, J.M., and Sheen, J. (2007). A central integrator of transcription networks in plant stress and cncrgy signalling. Naturc 448: 938942.

Baena-González, E., and Sheen, J. (2008). Convergent energy and stress signaling. Trends Plant Sci. 13: 474-482.

Bruneau, L., Chapman, R., and Marsolais, F. (2006). Co-occurrence of both L-asparaginase subtypes in Arabidopsis: At3g16150 encodes a K+-dependent L-asparaginase. Planta 224: 668-679.

Buchanan-Wollaston, V., Page, T., Harrison, E., Breeze, E., Lim, P. O., Nam, H.G., Lin, J.F., Wu, S.H., Swidzinski, J., Ishizaki, K., and Leaver, C.J. (2005). Comparative transcriptome analysis reveals significant differences in gene expression and signalling pathways between developmental and dark/starvation-induced senescence in Arabidopsis. Plant J. 42: 567-585.

Cortès, S., Gromova, M., Evrard, A., Roby, C., Heyraud, A., Rolin, D.B., Raymond, P., and Brouquisse, R.M. (2003). In plants, 3-omethylglucose is phosphorylated by hexokinase but not perceived as a sugar. Plant Physiol. 131: 824-837.

Diebold, R., Schuster, J., Däschner, K., and Binder, S. (2002). The branched-chain amino acid transaminase gene family in Arabidopsis encodes plastid and mitochondrial proteins. Plant Physiol. 129: 540-550.

Ehlert, A., Weltmeier, F., Wang, X., Mayer, C.S., Smeekens, S., Vicente-Carbajosa, J., and Dröge-Laser, W. (2006). Two-hybrid protein-protein interaction analysis in Arabidopsis protoplasts: establishment of a heterodimerization map of group $C$ and group $S$ bZIP transcription factors. Plant J. 46: 890-900.

Fode, B., and Gatz, C. (2009). Chromatin immunoprecipitation experiments to investigate in vivo binding of Arabidopsis transcription factors to target sequences. Methods Mol. Biol. 479: 261-272.

Gan, S. (2003). Mitotic and postmitotic senescence in plants. Sci. SAGE KE 2003: RE7.

Gibeaut, D.M., Hulett, J., Cramer, G.R., and Seemann, J.R. (1997). Maximal biomass of Arabidopsis thaliana using a simple, low-maintenance hydroponic method and favorable environmental conditions. Plant Physiol. 115: 317-319.

Gutiérrez, R.A., Stokes, T.L., Thum, K., Xu, X., Obertello, M., Katari, M.S., Tanurdzic, M., Dean, A., Nero, D.C., McClung, C.R., and Coruzzi, G.M. (2008). Systems approach identifies an organic nitrogen-responsive gene network that is regulated by the master clock control gene CCA1. Proc. Natl. Acad. Sci. USA 105: 4939-4944.

Hanson, J., Hanssen, M., Wiese, A., Hendriks, M.M.W.B., and Smeekens, S. (2008). The sucrose regulated transcription factor bZIP11 affects amino acid metabolism by regulating the expression of
ASPARAGINE SYNTHETASE1 and PROLINE DEHYDROGENASE2. Plant J. 53: 935-949.

Hellmann, H., Funck, D., Rentsch, D., and Frommer, W.B. (2000). Hypersensitivity of an Arabidopsis sugar signaling mutant toward exogenous proline application. Plant Physiol. 122: 357-368.

Hiratsu, K., Matsui, K., Koyama, T., and Ohme-Takagi, M. (2003). Dominant repression of target genes by chimeric repressors that include the EAR motif, a repression domain, in Arabidopsis. Plant $\mathrm{J}$. 34: 733-739.

Hruz, T., Laule, O., Szabo, G., Wessendorp, F., Bleuler, S., Oertle, L., Widmayer, P., Gruissem, W. and Zimmermann, P. (2008). Genevestigator V3: a reference expression database for the meta-analysis of transcriptomes. Adv. Bioinformatics 2008: 420747.

Jakoby, M., Weisshaar, B., Dröge-Laser, W., Vicente-Carbajosa, J., Tiedemann, J., Kroj, T., and Parcy, F.; bZIP Research Group (2002). bZIP transcription factors in Arabidopsis. Trends Plant Sci. 7: 106-111.

Kang, S.G., Price, J., Lin, P.C., Hong, J.C., and Jang, J.C. (2010). The Arabidopsis bZIP1 transcription factor is involved in sugar signaling, protein networking, and DNA binding. Mol. Plant 3: 361-373.

Kirby, J., and Kavanagh, T.A. (2002). NAN fusions: A synthetic sialidase reporter gene as a sensitive and versatile partner for GUS. Plant J. 32: 391-400.

Lam, H.M., Hsieh, M.H., and Coruzzi, G. (1998). Reciprocal regulation of distinct asparagine synthetase genes by light and metabolites in Arabidopsis thaliana. Plant J. 16: 345-353.

Lam, H.M., Peng, S.S., and Coruzzi, G.M. (1994). Metabolic regulation of the gene encoding glutamine-dependent asparagine synthetase in Arabidopsis thaliana. Plant Physiol. 106: 1347-1357.

Lam, H.M., Wong, P., Chan, H.K., Yam, K.M., Chen, L., Chow, C.M., and Coruzzi, G.M. (2003). Overexpression of the ASN1 gene enhances nitrogen status in seeds of Arabidopsis. Plant Physiol. 132: 926-935.

Lin, J.F., and Wu, S.H. (2004). Molecular events in senescing Arabidopsis leaves. Plant J. 39: 612-628.

Nakashima, K., Satoh, R., Kiyosue, T., Yamaguchi-Shinozaki, K., and Shinozaki, K. (1998). A gene encoding proline dehydrogenase is not only induced by proline and hypoosmolarity, but is also developmentally regulated in the reproductive organs of Arabidopsis. Plant Physiol. 118: 1233-1241.

Pilot, G., Stransky, H., Bushey, D.F., Pratelli, R., Ludewig, U., Wingate, V.P., and Frommer, W.B. (2004). Overexpression of GLUTAMINE DUMPER1 leads to hypersecretion of glutamine from hydathodes of Arabidopsis leaves. Plant Cell 16: 1827-1840.

Polge, C., and Thomas, M. (2007). SNF1/AMPK/SnRK1 kinases, global regulators at the heart of energy control? Trends Plant Sci. 12: 20-28.

Satoh, R., Fujita, Y., Nakashima, K., Shinozaki, K., and YamaguchiShinozaki, K. (2004). A novel subgroup of bZIP proteins functions as transcriptional activators in hypoosmolarity-responsive expression of the ProDH gene in Arabidopsis. Plant Cell Physiol. 45: 309-317.

Satoh, R., Nakashima, K., Seki, M., Shinozaki, K., and YamaguchiShinozaki, K. (2002). ACTCAT, a novel cis-acting element for prolineand hypoosmolarity-responsive expression of the ProDH gene encoding proline dehydrogenase in Arabidopsis. Plant Physiol. 130: 709-719.

Schuster, J., and Binder, S. (2005). The mitochondrial branched-chain aminotransferase (AtBCAT-1) is capable to initiate degradation of leucine, isoleucine and valine in almost all tissues in Arabidopsis thaliana. Plant Mol. Biol. 57: 241-254.

Sprenger-Haussels, M., and Weisshaar, B. (2000). Transactivation properties of parsley proline-rich bZIP transcription factors. Plant $\mathrm{J}$. 22: 1-8.

Usadel, B., Bläsing, O.E., Gibon, Y., Retzlaff, K., Höhne, M., Günther, 
M., and Stitt, M. (2008). Global transcript levels respond to small changes of the carbon status during progressive exhaustion of carbohydrates in Arabidopsis rosettes. Plant Physiol. 146: 1834-1861. van der Graaff, E., Schwacke, R., Schneider, A., Desimone, M., Flügge, U.I., and Kunze, R. (2006). Transcription analysis of arabidopsis membrane transporters and hormone pathways during developmental and induced leaf senescence. Plant Physiol. 141: 776-792. Weigel, R., and Glazebrook, J. (2002). Arabidopsis: A Laboratory

Manual. (Cold Spring Harbor, NY: Cold Spring Harbor Laboratory Press). Weltmeier, F., Ehlert, A., Mayer, C.S., Dietrich, K., Wang, X. Schütze, K., Alonso, R., Harter, K., Vicente-Carbajosa, J., and Dröge-Laser, W. (2006). Combinatorial control of Arabidopsis proline dehydrogenase transcription by specific heterodimerisation of bZIP transcription factors. EMBO J. 25: 3133-3143.
Weltmeier, F., et al. (2009). Expression patterns within the Arabidopsis $\mathrm{C} / \mathrm{S} 1$ bZIP transcription factor network: Availability of heterodimerization partners controls gene expression during stress response and development. Plant Mol. Biol. 69: 107-119.

Wiese, A., Elzinga, N., Wobbes, B., and Smeekens, S. (2004). A conserved upstream open reading frame mediates sucrose-induced repression of translation. Plant Cell 16: 1717-1729.

Wiese, A., Elzinga, N., Wobbes, B., and Smeekens, S. (2005). Sucrose-induced translational repression of plant bZIP-type transcription factors. Biochem. Soc. Trans. 33: 272-275.

Yakir, E., Hilman, D., Hassidim, M., and Green, R.M. (2007). CIRCADIAN CLOCK ASSOCIATED1 transcript stability and the entrainment of the circadian clock in Arabidopsis. Plant Physiol. 145: 925-932.

\section{Contributions:}

Figure 5A: Cloning of ProASN1:GUS / G-box mutated ProASN1:GUS reporter constructs

Figure 6A: Cloning and functional evaluation of p35S:HA-EAR repression vector

Figure S7: Cloning of ProProDH:GUS and ProASN1:GUS reporter constructs

Figure S8: Determination of energy starvation conditions for protoplasts by prolonged night and anoxia. 
Supplemental Data. Dietrich et al. (2011). Plant Cell 10.1105/tpc.110.075390

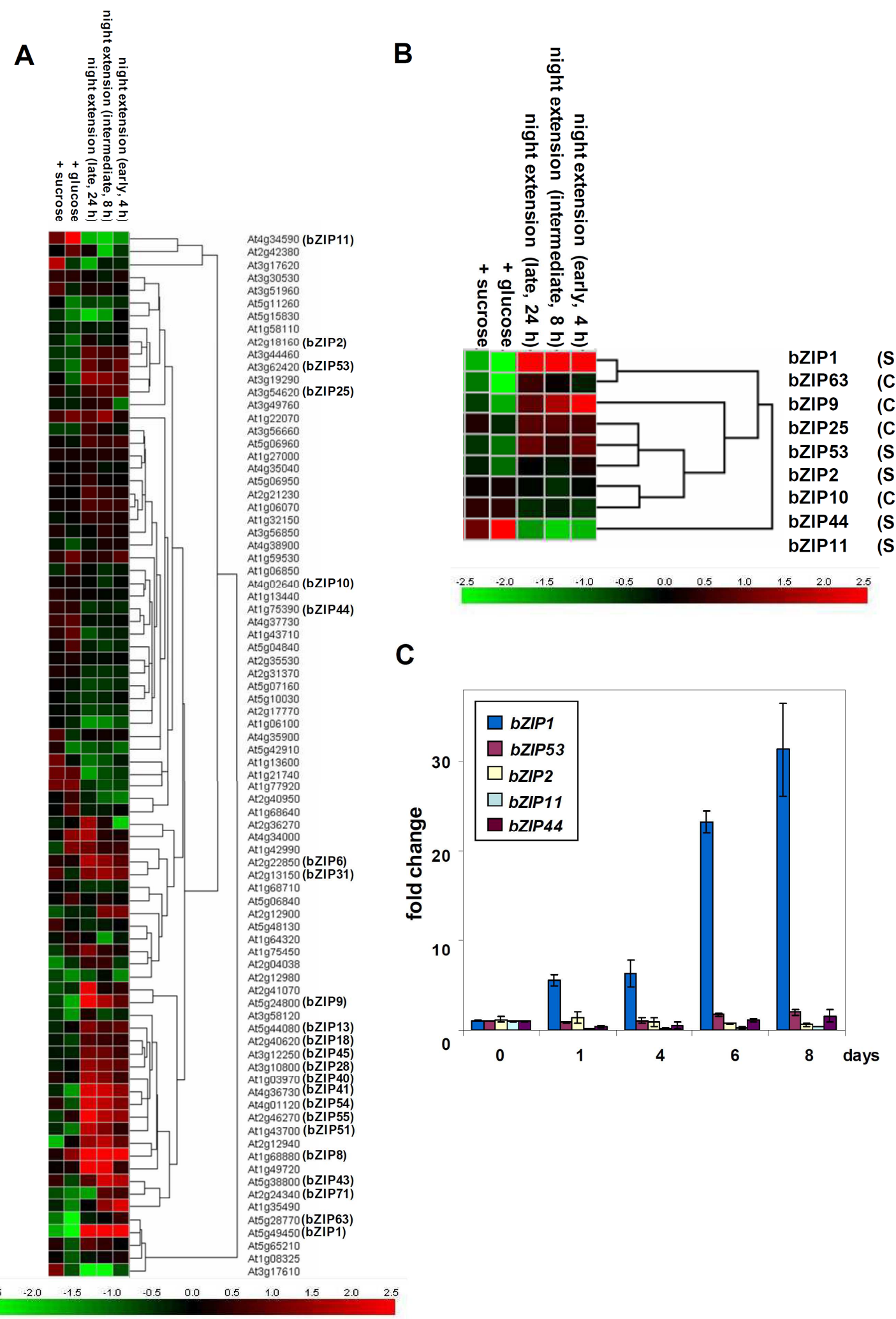


Supplemental Figure 1: Expression of bZIP genes during energy starvation. (A) Cluster analysis of all Arabidopsis bZIP genes (Jakoby et al., 2002) using the Genevestigator tool (Hruz et al., 2008). Transcriptome datasets analysing energy deprivation are compared and the bZIP TFs are clustered with respect to their expression profiles during early, intermediate and late extension of the normal $8 \mathrm{~h}$ night, respectively (time points as indicated). Opposed are treatments with glucose or sucrose (incubation for 4 h). Red and green colours show induction or repression of the corresponding genes as indicated in the colour bar beneath the heat map. (B) BZIP TFs of group S1 and C have been demonstrated to form specific heterodimers (Ehlert et al., 2006). Accordingly, cluster analysis was performed for the expression of the nine bZIP TFs during the treatments described in (A). (C) Transcript abundance of the group S1 bZIP TF genes after 0-8 days of dark treatment measured by qPCR analysis. Labelling of the bars are indicated. Rosette leaves of 10 three week-old plants have been pooled and used for RNA preparation and qPCR at the time points indicated. Depicted is the fold change compared to wt at $0 \mathrm{~h}$. Given are mean value and SD of two technical replicates.

Supplemental Data. Dietrich et al. (2011). Plant Cell 10.1105/tpc.110.075390

A

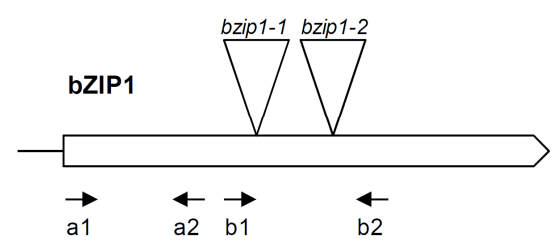

B
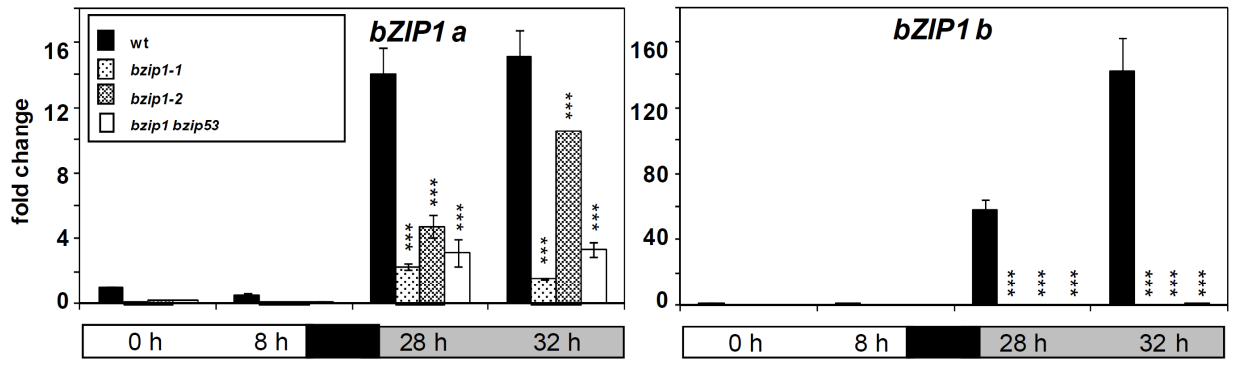

C

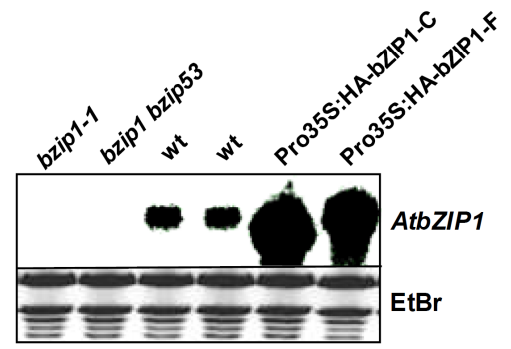

D

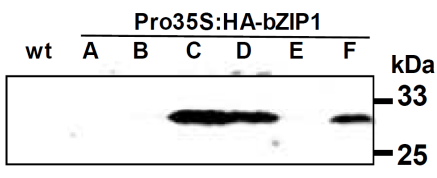

Supplemental Figure 2: Molecular characterization of transgenic plants with altered amounts of bZIP1.

(A) Schematic view showing location of the T-DNA insertion in the SALK lines bzipl-1 (SALK_059343) and bzip1-2 (SALK_069489). Given are the primers (arrows) used for the mutant 
characterisation provided in (B) which amplify fragments in the 5' (a1 and a2) or a 3' (b1 and b2) region of the gene. (B) Expression analysis of bZIPl in the T-DNA mutant lines bzipl-1, bzipl-2 and the double mutant bzipl bzip53 performed by qPCR using primer pair a (bZIP1a) or b (bZIP1b). For the double and quadruple mutants, the bzip1-1 allele has been used. The light/dark regime is given in the schematic drawing. Rosette leaves of 10 three week-old plants have been pooled and used for RNA preparation and qPCR at the time points indicated. Depicted is the fold change compared to wt at $0 \mathrm{~h}$. Given are mean value and SD of two technical replicates. Asterisks represent significant differences of mutant plants compared to the wt at the indicated time point (two-way ANOVA, * p $<0.05 ; * * \mathrm{p}<0.01$; *** $\mathrm{p}<$ 0.001). (C) RNA blot analysis of bZIP1-specific transcripts using wt, T-DNA insertion lines (bzip1-1, bzip1 bzip53) and plants overexpressing bZIP1 under control of the 35S promoter (Pro35S:HA-bZIP1). Ethidium bromide staining (EtBr) was used as a loading control. (D) Immunoblot analysis of protein extracts derived from the Pro35S:HAbZIP1 lines indicated using a HA-tag specific antibody. Line C was been used in the experiments described.

Supplemental Data. Dietrich et al. (2011). Plant Cell 10.1105/tpc.110.075390

Dietrich et al., Function of bZIP factors in starvation response
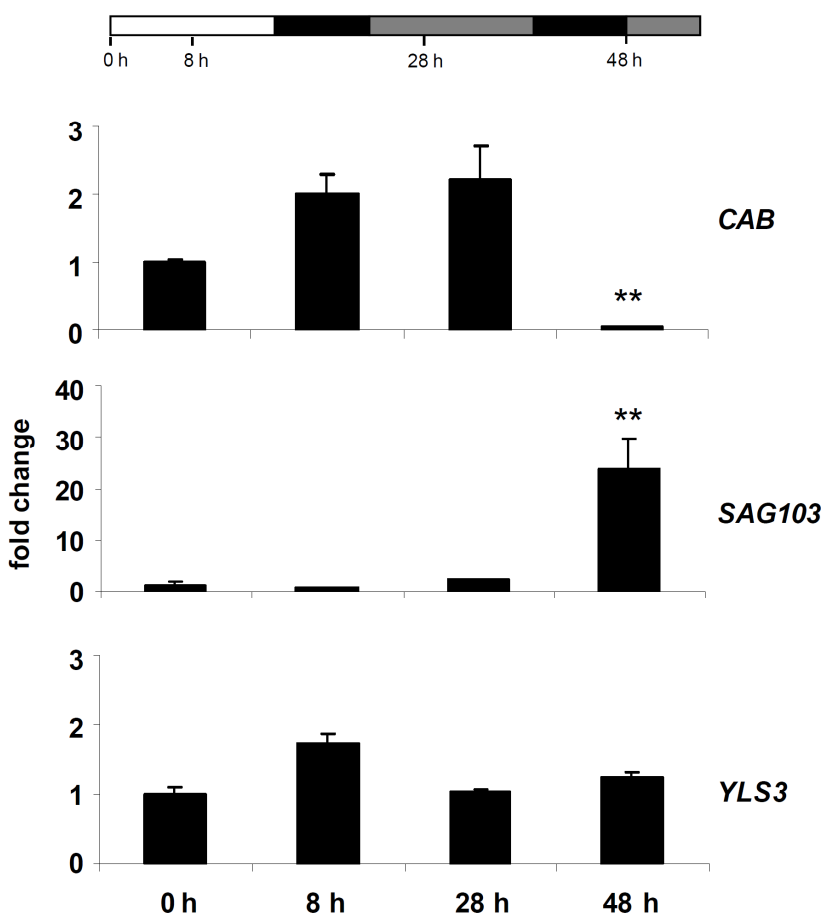

Supplemental Figure 3: Expression of senescence marker genes was analysed in wt plants cultivated under extended night conditions. Day, night and extended night phases are indicated by white, black or grey bars, respectively. RNA isolated at the time points indicated was analyzed by qPCR. Whereas transcription of photosynthetic markers such as CAB is strongly reduced at the $48 \mathrm{~h}$ timepoint, SAG103 
as a marker for dark-induced senescence related to energy deprivation (van der Graaff et al., 2006) is strongly induced in wt plants. This process is distinct from natural senescence as the expression of the corresponding marker gene (YLS3) is not significantly changed. Rosette leaves of 10 three week-old plants have been pooled and used for RNA preparation and qPCR at the time points indicated. Depicted is the fold change compared to wt at $0 \mathrm{~h}$. Given are mean value and SD of two technical replicates. Asterisks (**) indicate values that were determined by one-way ANOVA and following Tukey's post-test to be significantly different $(\mathrm{p}<0.01)$ in comparison to the other time points.

Supplemental Data. Dietrich et al. (2011). Plant Cell 10.1105/tpc.110.075390

Dietrich et al., Function of bZIP factors in starvation response
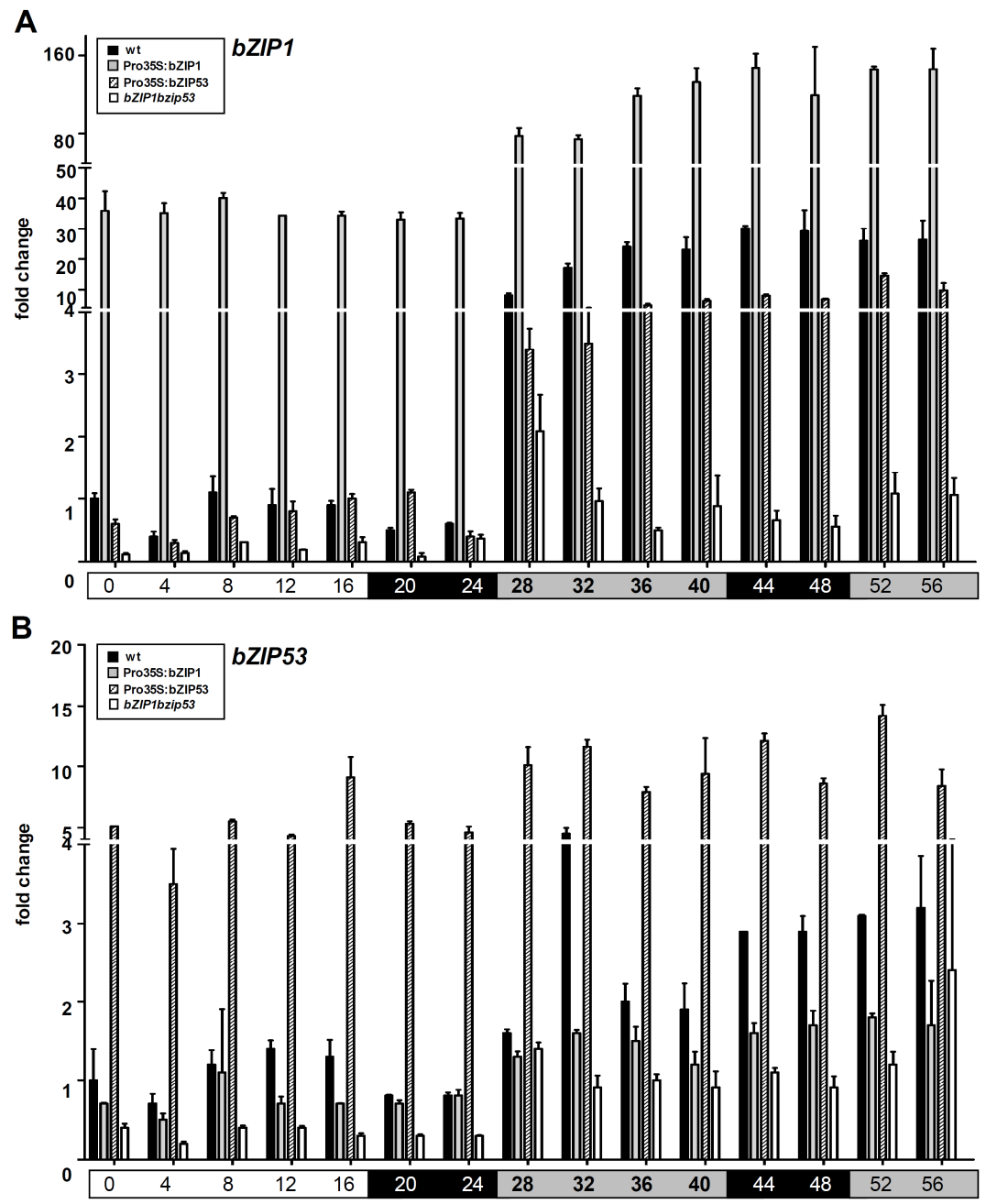

Supplemental Figure 4: Expression of bZIPI and bZIP53 after extended night treatment. Wt (black bars), overexpression plants under control of the $35 \mathrm{~S}$ promoter (Pro35S:bZIP1, grey bars; Pro35S:bZIP53, hatched bars) and a double T-DNA insertion line (bzipl bzip53, white bars) were 
compared by qPCR with respect to bZIP1 (A) and bZIP53 (B) transcript abundance as described in Figure 1. Due to insertion of the T-DNA in the promoter region, bzip53 is considered to be a knock-down, (Weltmeier et al., 2006), whereas no significant amounts of bzipl transcript are observed. Rosette leaves of 10 three week-old plants have been pooled and used for RNA preparation and qPCR at the time points indicated. Depicted is the fold change compared to wt at $0 \mathrm{~h}$. Given are mean value and SD of two technical replicates. For visualizing the differences in transcript levels, the y-axis is broken.

Supplemental Data. Dietrich et al. (2011). Plant Cell 10.1105/tpc.110.075390

Dietrich et al., Function of bZIP factors in starvation response

A
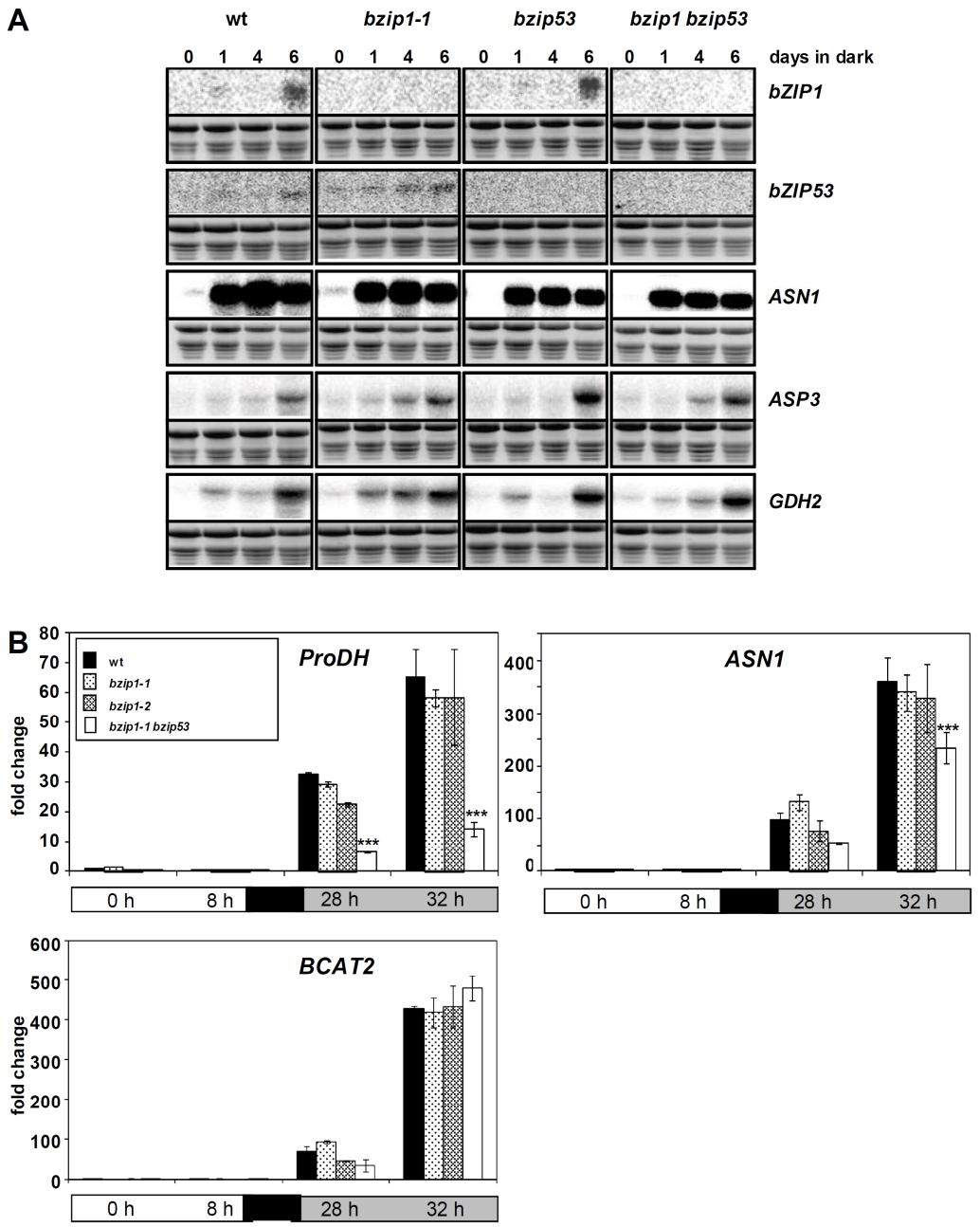

Supplemental Figure 5: Expression analysis of genes involved in amino acid metabolism in single and double T-DNA insertion mutants of bZIP1 and bZIP53 (A) RNA blot analysis of the indicated genes after long-term dark treatment for 0 - 6 days. Compared are wt, bzip1-1, bzip53 and bzipl bzip53 plants. As a loading control, ethidium bromide (EtBr) stainings are provided for each hybridisation experiment. (B) qPCR expression analysis of $A S N 1$, ProDH and BCAT2 in the two T-DNA mutant lines bzipl-1 and 
bzip1-2 and in the double mutant bzipl bzip53. The light/dark regime is given in the schematic drawing. Rosette leaves of 10 three week-old plants have been pooled and used for RNA preparation and qPCR at the time points indicated. Depicted is the fold change compared to wt at $0 \mathrm{~h}$. Given are mean value and SD of two technical replicates. Asterisks represent significant differences of mutant plants compared to the wt at the indicated time point (two-way ANOVA, * $\mathrm{p}<0.05 ; * * \mathrm{p}<0.01 ; * * * \mathrm{p}<0.001$ ).

Supplemental Data. Dietrich et al. (2011). Plant Cell 10.1105/tpc.110.075390

A

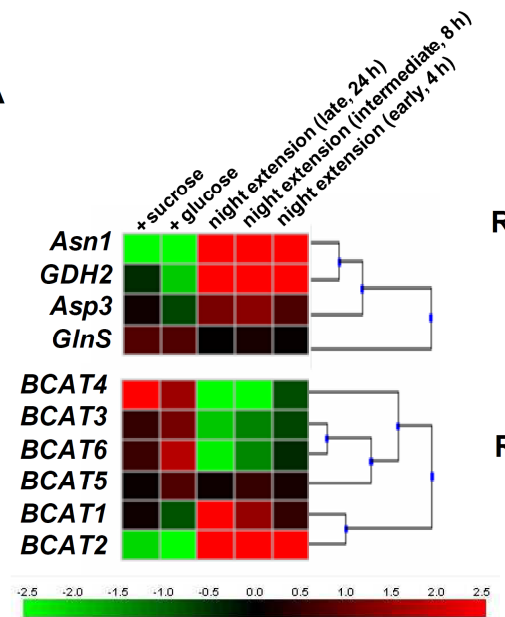

C

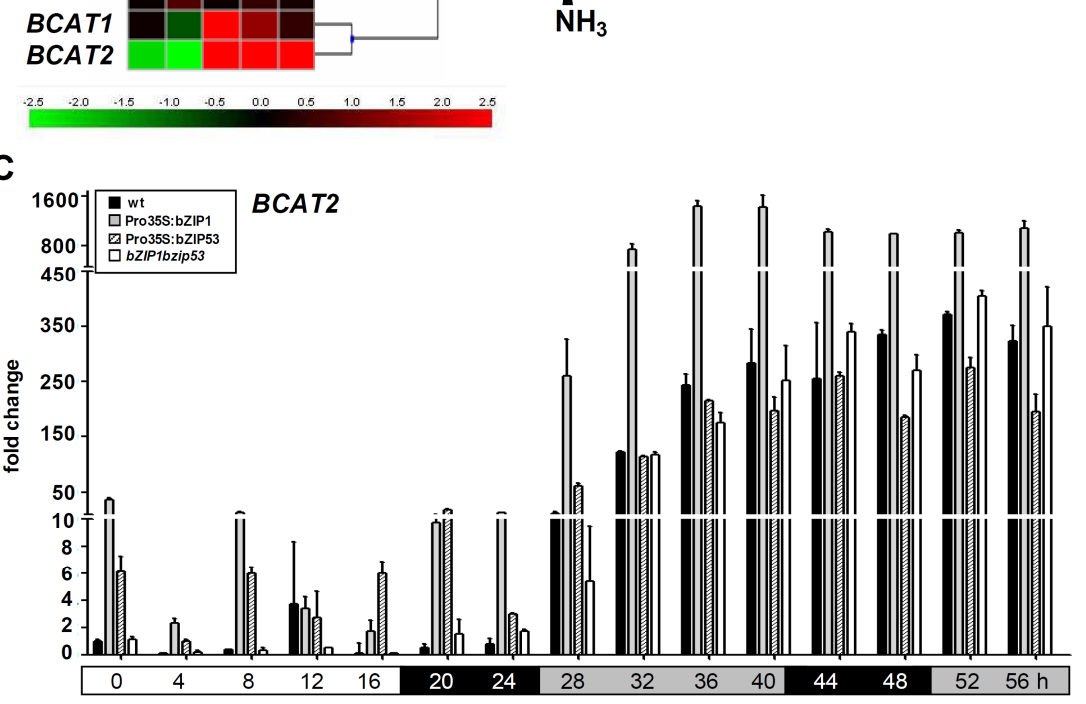

B
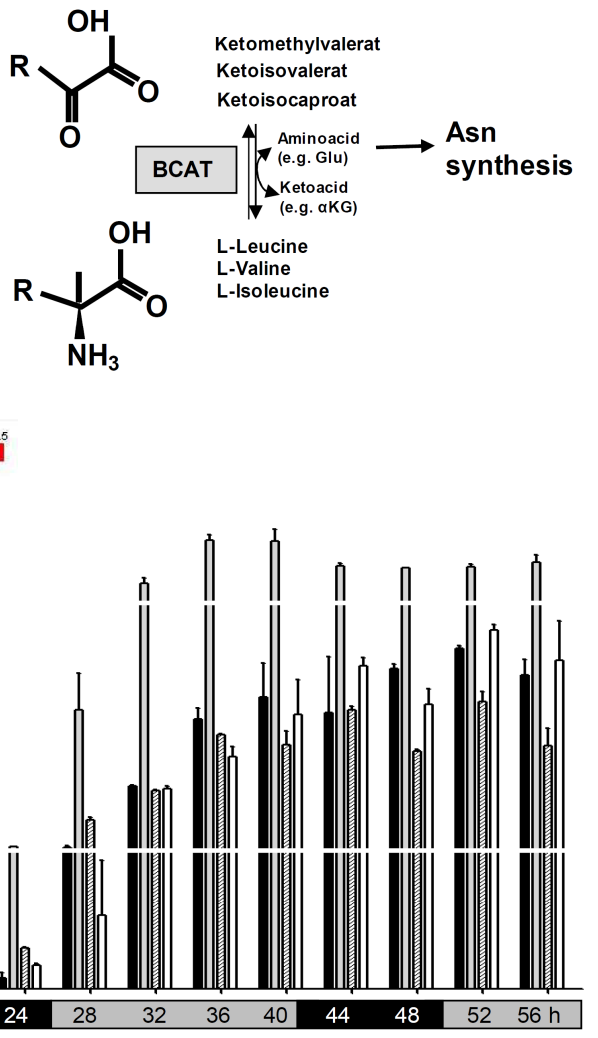

Supplemental Figure 6: Regulation of transcription in $\underline{\text { Branched }} \underline{\text { Chain }} \underline{\mathrm{A} m i n o} \underline{\mathrm{A}} \mathrm{cid}$ (BCAA) metabolism during dark-treatment. (A) Cluster analysis of the Asn biosynthesis and BCAT genes indicated, making use of public expression data and the Genevestigator tool (Hruz et al., 2008). Transcriptome data sets analysing energy deprivation are compared at early, intermediate and late night extension ( $\mathrm{h}$ as indicated). Opposed are treatments with glucose or sucrose (incubation for $4 \mathrm{~h}$ ), respectively. Red and green coloursshow induction or repression of the corresponding genes as indicated in the colour bar beneath the heat map. (B) Schematic overview of BCAA metabolism. $\alpha$ KG, $\alpha$-keto- 
glutarate. (C) Induction of BCAT2 after short-term dark treatment. Wt (black bars), Pro35S:bZIP1 (grey bars), Pro35S:bZIP53 (hatched bars) and the corresponding T-DNA insertion line (bzip1 bzip53, white bars) have been cultivated at a day/night rhythm of $16 / 8 \mathrm{~h}$ as indicated by the scheme at the bottom. Day, night and extended night phases are indicated by white, black or grey bars, respectively. Rosette leaves of 10 three week-old plants have been pooled and used for RNA preparation and qPCR at the time points indicated. Depicted is the fold change compared to wt at $0 \mathrm{~h}$. Given are mean value and SD of two technical replicates. For visualizing the differences in transcript levels, the y-axis is broken at 10 and 450 fold induction.

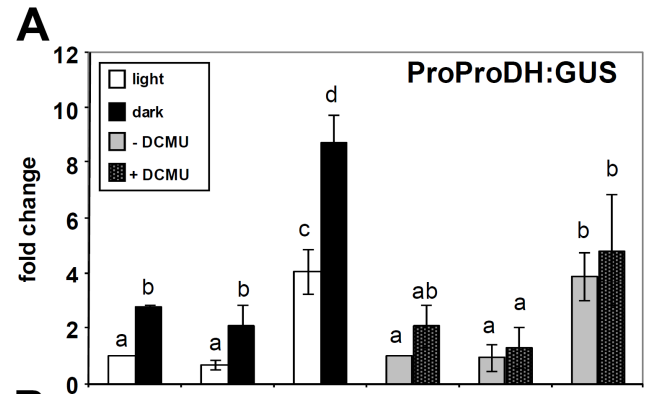

B

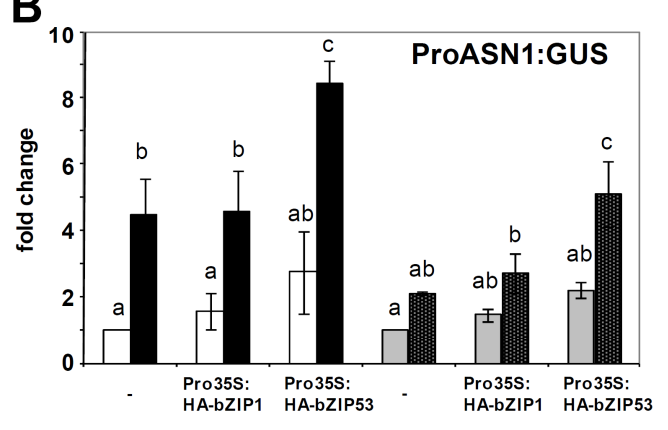

C

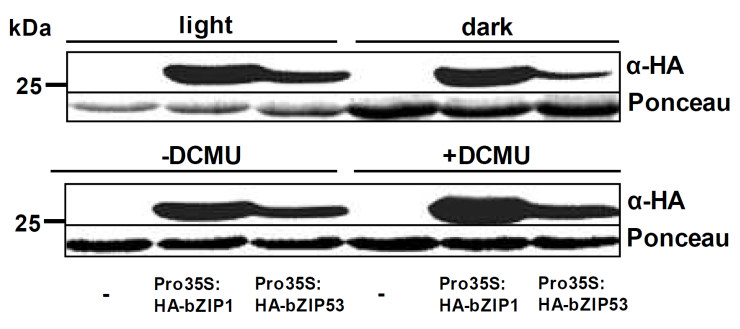

Supplemental Figure 7: Analysis of energy deprivation-induced transcription in protoplasts. Promoter:GUS reporter constructs of the bZIP target genes, namely ProDH (A) and ASN1 (B), were used in transiently transformed protoplast assays. Induction of the reporters has been observed after constant dark treatment (black bars) in comparison to constant light treatment (white bars) for $16 \mathrm{~h}$. Comparable induction was observed by treatment with the photosystem II inhibitor DCMU (dark grey bars) in 
comparison to untreated cells (light grey bars). GUS activity was determined after co-transformation with $14 \mu \mathrm{g}$ of Pro35S:HA-bZIP1 or Pro35S:HA-bZIP53 effector plasmids or an empty vector control (-). Inclusion of $1 \mu \mathrm{g}$ of a reference plasmid encoding a Pro35S:NAN gene was used in all experiments to normalize GUS expression values for differences in transfection efficiencies as described by (Ehlert et al., 2006). Y-axis values are expressed as fold change in comparison to the control incubation under constant light. Given are mean values and SD of four independent transfections. Different letters indicate significant differences of light/dark and +/- DCMU treatment, tested by one-way ANOVA analysis following Tukey's post-test, $\mathrm{p}<0.05$, respectively. (C) Expression of the HA-tagged bZIP effectors was studied using immunoblot analysis in light/dark grown or DCMU treated/untreated protoplasts using a HAtag specific antibody ( $\alpha$ HA). For loading control, Ponceau staining was used. Protein size is compared to size standard in kilo Dalton $(\mathrm{kDa})$.

Supplemental Data. Dietrich et al. (2011). Plant Cell 10.1105/tpc.110.075390

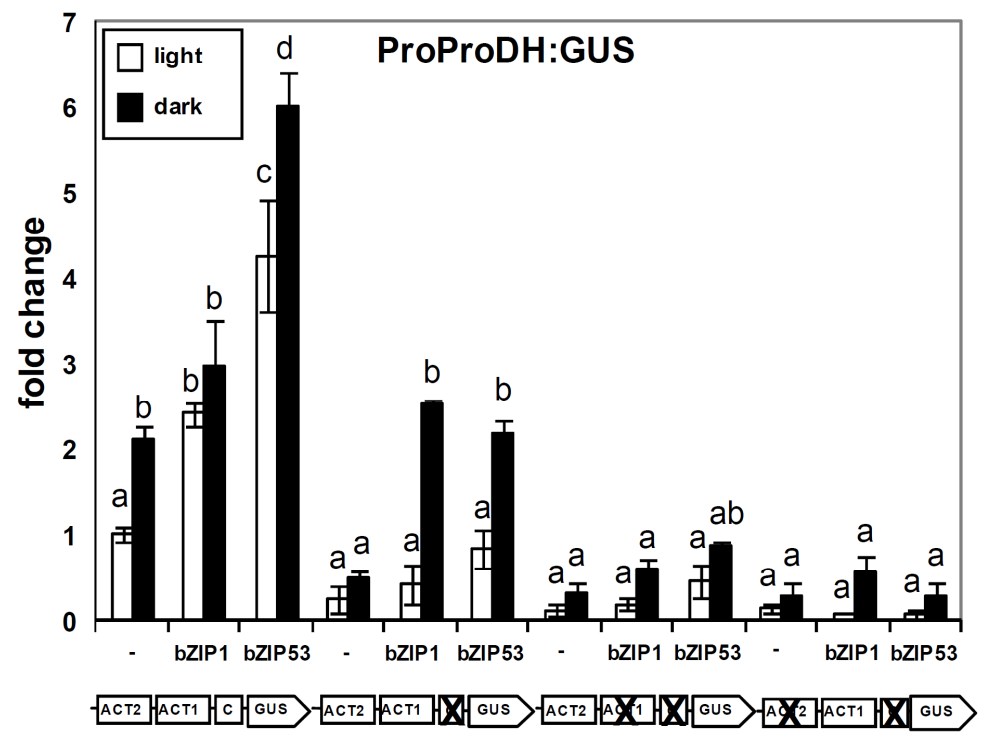

Supplemental Figure 8: Mutation in the C-box affects ProDH promoter activity. Transient transformation of protoplasts was performed as described in Figure 5 with the promoter mutations indicated (diagram below x-axis). Depicted is the fold change compared to the unmutated promoter in the light. Given are mean values and SD of four independent transfections. Different letters indicate significant differences, tested by One-way ANOVA analysis following Tukey's post-test, $\mathrm{p}<0.05$. 
Supplemental Data. Dietrich et al. (2011). Plant Cell 10.1105/tpc.110.075390

A

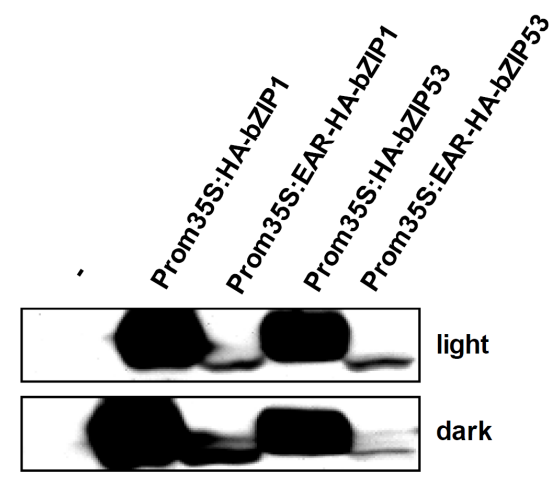

B

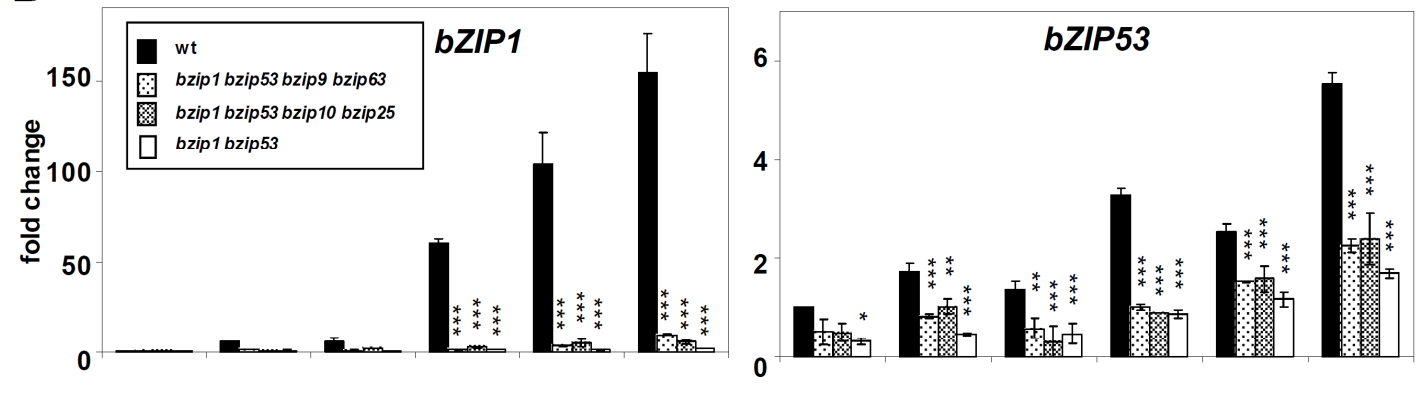

C

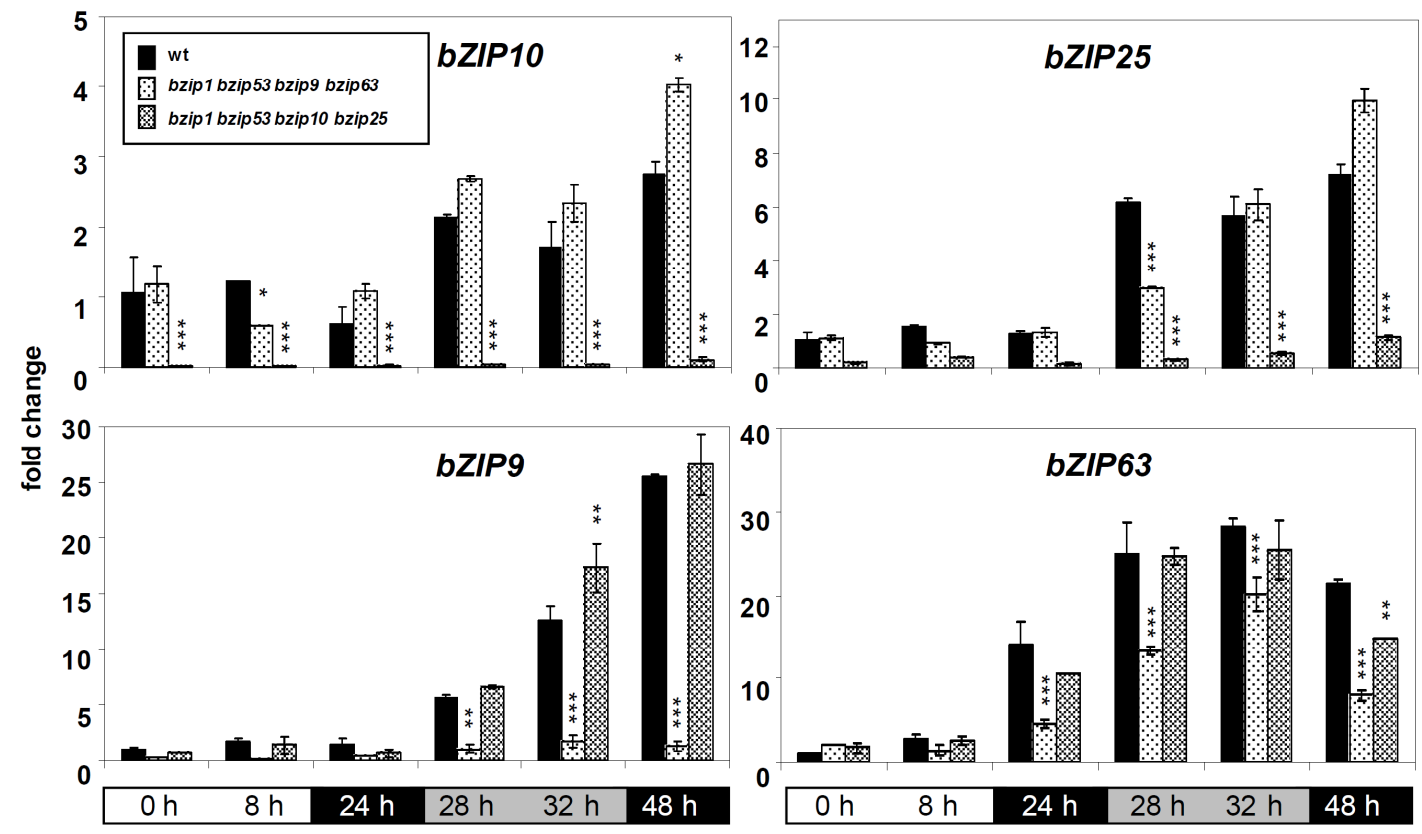

Supplemental Figure 9: Immunoblot analysis of the EAR repressor approach and characterisation of the multiple T-DNA insertion mutants used in this study. (A) The expression of HA-bZIP1, HA-bZIP53, EAR-HA-bZIP1 and EAR-HAbZIP53 in Figure 6A in constant light (upper panel) or darkness (lower 
panel) was monitored by immunoblot analysis using an $\alpha$-HA antibody. Since the EAR domain fusions harbour only two HA epitopes in comparison to the constructs without EAR domain (three epitopes), the expression levels can only be compared between the related protein fusions. (B) Expression analysis of bZIP1 and bZIP53 performed by qPCR using the indicated T-DNA insertion lines. (C) Expression analysis of group C bZIP10, bZIP25, bZIP9 and bZIP63 using the indicated T-DNA insertion lines. Rosette leaves of 10 three week-old plants have been pooled and used for RNA preparation and qPCR at the time points indicated. Depicted is the fold change compared to wt at $0 \mathrm{~h}$. Given are mean value and SD of two technical replicates. Asterisks represent significant differences between mutant plants and the wt at the indicated time point (two-way ANOVA, * $\mathrm{p}<0.05 ; * * \mathrm{p}<0.01 ; * * * \mathrm{p}<0.001$ ).

Supplemental Table 1: Summary of putative bZIP binding motifs (ACGT- like elements) found in the promoters ( $1 \mathrm{~kb}$ upstream region) of genes related to Asn, Pro and BCAT metabolism. Genes, AGI code and the putative elements are given.

\begin{tabular}{|c|c|c|c|}
\hline Gene & AGI code & $\begin{array}{l}\text { ACGT-like elements } \\
\text { (-1000 bp promoter region) }\end{array}$ & Box \\
\hline ProDH & At3g30775 & $\begin{array}{l}-842 \text { cACTCATcct }-833 \\
-714 \text { gACTCATcct }-705 \\
-683 \text { ctgACGTcct }-674 \\
-572 \text { ataACGTagc }-563 \\
-197 \text { aaaACGTgta }-188 \\
-171 \text { ttACGTgct }-162\end{array}$ & $\begin{array}{l}\text { ACT2 } \\
\text { ACT1 } \\
\text { C } \\
\text { T/A } \\
\text { T/G } \\
\text { A/G }\end{array}$ \\
\hline ASN1 & At3g47340 & $\begin{array}{l}-731 \text { ttaACGTttt }-722 \\
-557 \text { aacACGTgta }-548 \\
-377 \text { cggACGTcgt }-368 \\
-354 \text { aacACGTgga }-345 \\
-167 \text { tctACGTgca }-158\end{array}$ & $\begin{array}{l}T \\
G 2 \\
C \\
G 1 \\
A / G\end{array}$ \\
\hline GDH2 & At5g07440 & $\begin{array}{l}-974 \text { ctaACGTgga }-965 \\
-737 \text { tccACGTccc }-728 \\
-282 \text { ctgACGTagg }-273 \\
-273 \text { gatACGTcaa }-264\end{array}$ & $\begin{array}{l}T / G \\
G / C \\
C / A \\
A / C\end{array}$ \\
\hline ASP3 & At5g11520 & $\begin{array}{l}-841 \text { tcaACGTggt }-832 \\
-480 \text { tatACGTtga }-471 \\
-122 \text { cttACGTggc }-113\end{array}$ & $\begin{array}{l}\mathrm{T} / \mathrm{G} \\
\mathrm{A} / \mathrm{T} \\
\mathrm{A} / \mathrm{G}\end{array}$ \\
\hline GLNS & At5g37600 & $-157 \mathrm{ttcACGTcac}-148$ & $\mathrm{G} / \mathrm{C}$ \\
\hline PepCK & At5g65690 & $\begin{array}{l}-738 \text { aaaACGTgct }-729 \\
-524 \text { caaACGTgaa }-515 \\
-467 \text { tgcACGTaaa }-458 \\
-423 \text { tcaACGTaat }-414 \\
-334 \text { ataACGTgag }-325 \\
-76 \text { tatACGTgta }-67\end{array}$ & $\begin{array}{l}T / G \\
T / G \\
G / A \\
T / A \\
T / G \\
A / G\end{array}$ \\
\hline ANS & At3g16150 & $\begin{array}{l}-882 \text { attACGTaag }-873 \\
-776 \text { ttgACGTata }-767 \\
-752 \text { ataACGTaga }-743 \\
-511 \text { ctgACGTgga }-502 \\
-361 \text { ataACGTttc }-352\end{array}$ & $\begin{array}{l}A \\
C / A \\
T / A \\
C / G \\
T\end{array}$ \\
\hline BCAT2 & At1g10070 & $\begin{array}{l}-804 \text { aaaACGTtgt }-795 \\
-770 \text { ctgACGTggc }-761 \\
-749 \text { acaACGTgtt }-740 \\
-712 \text { aagACGTtga }-703 \\
-633 \text { ccaACGTggc }-624 \\
-337 \text { gacACGTcta }-328 \\
-164 \text { cttACGTgtt }-155\end{array}$ & $\begin{array}{l}T \\
C / G \\
T / G \\
C / T \\
T / G \\
G / C \\
A / G\end{array}$ \\
\hline
\end{tabular}


Supplemental Table 2: Summary of the T-DNA insertion lines used in this study.

\begin{tabular}{|l|l|l|l|}
\hline \multicolumn{1}{|c|}{ Mutant } & SALK Nr. & Insertion in & $\begin{array}{l}\text { Knock down / } \\
\text { Knock out }\end{array}$ \\
\hline bzip1-1 & SALK_059343 & ORF & Knock out \\
\hline bzip1-2 & SALK_069489 & ORF & Knock out \\
\hline bzip53 & SALK_069883 & 5'UTR & Knock down \\
\hline bzip1 bzip53 & SALK_059343 & ORF & Knock out \\
& SALK_069883 & 5'UTR & Knock down \\
\hline bzip1 bzip53 & SALK_059343 & ORF & Knock out \\
bzip9 bzip63 & SALK_069883 & 5'UTR & Knock down \\
& SALK_093416 & 3. Exon & Knock out \\
& SALK_066531 & 5'UTR & Knock down \\
\hline bzip1 bzip53 & SALK_059343 & ORF & Knock out \\
bzip10 bzip25 & SALK_069883 & 5'UTR & Knock down \\
& SALK_106031 & 3. Exon & Knock out \\
& SALK_119931 & 1. Exon & Knock out \\
\hline
\end{tabular}

Supplemental Table 3: Summary of oligonucleotide primers used in this study. qPCR Primers:

\begin{tabular}{|c|c|c|}
\hline SAG103 & At1g10140 & $\begin{array}{l}\text { 5'-AGCTCGAGTGCTGGGATG-3' } \\
\text { 5'-CGGATTCACAGATCCTTCCT-3' }\end{array}$ \\
\hline $\mathrm{CAB}$ & At1g29920 & $\begin{array}{l}\text { 5'-TCAATCTTTTGAATTCGAGTGAGA-3' } \\
\text { 5'-TCCACCACAAACACAAACCTAC-3' }\end{array}$ \\
\hline YLS3 & At2g44290 & $\begin{array}{l}\text { 5'-GACATCACTAAGTGCCCTGCT-3' } \\
\text { 5'-ACTGTTTCGTTCAGACCTTTAGC-3' }\end{array}$ \\
\hline ASN1 & At3g47340 & $\begin{array}{l}\text { 5'-TTCTTGAGCTTTCTCGCAGAT-3' } \\
\text { 5'-CCGTTCTGATATAAGCCACTCC-3' }\end{array}$ \\
\hline ProDH & At3g30775 & $\begin{array}{l}\text { 5'-CGCCAGTCCACGACACAATTCA-3' } \\
\text { 5'-CGAATCAGCGTTATGTGTTGCG-3' }\end{array}$ \\
\hline ANS & At3g16150 & $\begin{array}{l}\text { 5'-TTCCGATCAGCATTTACGC-3' } \\
\text { 5'-CAATGTCCTTTCCCGTCAAC-3' }\end{array}$ \\
\hline BCAT2 & At1g10070 & QuantiTect $®$ Primer QT00854875 \\
\hline $\begin{array}{l}\text { bZIP1 a1 } \\
\text { bZIP1 a2 }\end{array}$ & At5g49450 & $\begin{array}{l}5^{\prime} \text {-TGGCAAACGCAGAGAAGACAAGT-3‘ } \\
5^{\prime} \text {-CCGCGAATCTGCAAGGAGGTCG-3، }\end{array}$ \\
\hline $\begin{array}{l}\text { bZIP1 b1 } \\
\text { bZIP1 b2 }\end{array}$ & At5g49450 & $\begin{array}{l}\text { 5'-GAGTTTAACGCTGACGCAGA-3' } \\
\text { 5'-GGAATAGCGGTTGGAGATTG-3' }\end{array}$ \\
\hline bZIP1 & At5g49450 & $\begin{array}{l}\text { 5'-TCAGCGTTAAACTCGTCGTAGCAA-3' } \\
\text { 5'-AACGCGGGTCTTAGATCGGAGAAG-3' }\end{array}$ \\
\hline bZIP53 & At3g62420 & $\begin{array}{l}\text { 5'-TGGGGTCGTTGCAAATGCAAACAA-3' } \\
\text { 5'-CCGTGGCGTACCTCGGATCATTAT-3' }\end{array}$ \\
\hline bZIP9 & At5g24800 & $\begin{array}{l}\text { 5'-CGAAAAGGTCCAGCCGGAAACAAT-3' } \\
\text { 5'-TCGACCTCATGAACCGGGATTACA-3' }\end{array}$ \\
\hline bZIP10 & At4g02640 & $\begin{array}{l}\text { 5'-TTTTTCGGCCATGCTGAATCGTTC-3' } \\
\text { 5'-TTACTCCAAGCGCCAACCCGTA-3' }\end{array}$ \\
\hline bZIP25 & At3g54620 & $\begin{array}{l}\text { 5'-AGGAGGATGCTCTCAAACCGAGAA-3' } \\
\text { 5'-CGGCTCTTAATTGGCCTACCTGTG-3' }\end{array}$ \\
\hline bZIP63 & At5g28770 & $\begin{array}{l}\text { 5'-AAAAGGGGAACTTTCATCAAACCTCAGG-3' } \\
\text { 5'-CTGAGGCATATTGTGGAACATTGGGT-3' }\end{array}$ \\
\hline UBQ5 & At3g62250 & $\begin{array}{l}\text { 5'-GACGCTTCATCTCGTCC-3' } \\
\text { 5'-GTAAACGTAGGTGAGTCCA-3' }\end{array}$ \\
\hline
\end{tabular}


ChIP Primers:

\begin{tabular}{|c|c|l|}
\hline ASN1 & At3g47340 & $\begin{array}{l}\text { 5'-CACGTGTACGGCTCTAAAGCA-3' } \\
\text { 5'-GACCAGCTGTTTCCACGTGTT-3' }\end{array}$ \\
\hline ProDH & At3g30775 & 5'-CAAGTCCAGGTCCACATGTTG-3' \\
& & 5'-CAAGGCCCTGACCATAGGA-3' \\
\hline ACT7 & At5g09810 & 5'-CGTTTCGCTTTCCTTAGTGTTAGCT-3' \\
& & 5'-AGCGAACGGATCTAGAGACTCACCTTG-3' \\
\hline
\end{tabular}

Primers to test of T-DNA Insertion:

\begin{tabular}{|c|c|l|}
\hline bZIP1 & At5g49450 & $\begin{array}{l}\text { 5'-ATGGCAAACGCAGAGAAGACAAGTTCA-3' } \\
\text { 5'-TCATGTCTTAAAGGACGCCATTGGTTG-3' }\end{array}$ \\
\hline bZIP53 & At3g62420 & 5'-CTGCAGCTTAGGACAGCTCATCACCA-3' \\
& & 5'-GTCGACCTCGTTGACTTTTTGACTTC-3' \\
\hline bZIP9 & At5g24800 & 5'-ATGGATAATCACACAGCTAAAGA -3' \\
& & 5'-GAGTTATCGCCTTTCAAAGA-3' \\
\hline bZIP10 & At4g02640 & 5'-TTGAGATGAGTTCTGGTGGTTA-3' \\
& & 5'-GTCCACTTGATCTTCCGAGAA-3' \\
\hline bZIP25 & At3g54620 & 5'-ATGCACATCGTCTTCTCTGTCG-3' \\
& & 5'-TGCCTTGTTTCTTTCTTCTCAG-3' \\
\hline bZIP63 & At5g28770 & 5'-CGTAGCTCGGTCATTGTGTCGG-3' \\
& & 5'-TCTCCGTCGTCTGCAGCGGC-3' \\
\hline LBa1 & & 5'-TGGTTCACGTAGTGGGCCATCG -3' \\
\hline
\end{tabular}

Primers to generate RNA blot probes:

\begin{tabular}{|c|c|l|}
\hline ASN1 & At3g47340 & $\begin{array}{l}\text { 5'-GTGGAATACTTGCCGTGTTAG-3' } \\
\text { 5'-CGAAATGCTCACAGTCATCG-3' }\end{array}$ \\
\hline GDH2 & At5g07440 & 5'-GCTGCAACAAACAGAAACTTCC-3' \\
& & 5'-CCCTGAATCGATTTCCCGT-3' \\
\hline ProDH & At3g30775 & 5'-ATGGCAACCCGTCTTCTCCG-3' \\
& & 5'-TGGTCGGTCTTTGTCAGCAT-3' \\
\hline BCAT2 & At1g10070 & 5'-CACATCTCTACGCAAAACTCTG-3' \\
& & 5'-TGTGATGCTCTTGACGCCTC-3' \\
\hline bZIP1 & At5g49450 & 5'-GGCAAACGCAGAGAAGACAA-3' \\
& & 5'-GGACGCCATTGGTTGTAGAG-3' \\
\hline bZIP53 & At3g62420 & 5'-GTGCTTGAGATGGTTGAAGAAATTA-3' \\
& & 5'-CATACAAAGACACGAACAAATTGAA-3' \\
\hline PepCK & At5g65690 & 5'-ATGGCGGGAAACGGAAACGA-3' \\
& & 5'-TCGTACAACTCGGCGGGAGA-3' \\
\hline ASP3 & At5g11520 & 5'-CTTCCTCTTCTTCTTCCGATCG-3' \\
& & 5'-GTCGCTGGATCGTAGTATCGG-3' \\
\hline GLNS & At5g37600 & 5'-CATCAACCTTAACCTCTCAGACTCCA-3' \\
& & 5'-CTCGTAACCGCCTTCTTCCCTCATTG-3' \\
\hline
\end{tabular}


Primers for construction of promoter:GUS constructs:

\begin{tabular}{|c|c|c|}
\hline ASN1 promoter & $\begin{array}{l}\text { with Xbal und } \mathrm{Ncol} \\
\text { restriction sites }\end{array}$ & $\begin{array}{l}\text { 5'AAAAAAAAAATCTAGACGTCTCAAGTATT } \\
\text { GATAAAATAATAAT-3' } \\
\text { 5'-AAAAAAAAAACCATGGGTTTTTTTTTTTGAA } \\
\text { GAAAGTGAAAAAGATCACGAAGAA-3' }\end{array}$ \\
\hline $\begin{array}{l}\text { ProDH } \\
\text { promoter }\end{array}$ & $\begin{array}{l}\text { with Pstl and Ncol } \\
\text { restriction sites }\end{array}$ & $\begin{array}{l}\text { 5'-AAAAAAAAAACTGCAGACTTGCACTAAA } \\
\text { GACTAAACAGT-3' } \\
\text { 5'-AAAAAAAAAACCATGGAAAATTCAAAGATT } \\
\text { TTGTTTTTGAAAACGAAAACAAA-3' }\end{array}$ \\
\hline $\begin{array}{l}\text { ProDH } \\
\text { promoter } \\
\text { ACTCAT1 mut }\end{array}$ & ACTCAT1 mutation & $\begin{array}{l}\text { 5'-TATATTAATTAAATAGTTATCCGATGTTAC } \\
\text { ATCCTATGGTCAGGGCCTTGCTGTC-3' } \\
\text { 5'-GACAGCAAGGCCCTGACCATAGGATGTA } \\
\text { ACATCGGATAACTATTTAATTAATATA-3' }\end{array}$ \\
\hline $\begin{array}{l}\text { ProDH } \\
\text { promoter } \\
\text { ACTCAT2 mut }\end{array}$ & ACTCAT2 mutation & $\begin{array}{l}\text { 5'-ATGTTGAATCCGATTCATCATCCTTACAT } \\
\text { CCTTCATATCTTCCTCCACC-3' } \\
\text { 5'-GGTGGAGGAAGATATGAAGGATGTAAG } \\
\text { GATGATGAATCGGATTCAACAT-3' }\end{array}$ \\
\hline $\begin{array}{l}\text { ProDH } \\
\text { promoter C-box } \\
\text { mut }\end{array}$ & C-box mutation & $\begin{array}{l}\text { 5'-CTATGGTCAGGGCCTTGCTGTCTCTGAAA } \\
\text { TCCTTAATTATCATTAT-3' } \\
\text { 5'-ATAATGATAATTAAGGATTTCAGAGACAG } \\
\text { CAAGGCCCTGACCATAG-3' }\end{array}$ \\
\hline $\begin{array}{l}\text { ASN1 promoter } \\
\text { G1-box mut }\end{array}$ & G1 mutation & $\begin{array}{l}\text { 5'-CTAGTCTAGAGCAGGCTCATTTCGCGGA } \\
\text { AAGTTG-3' } \\
\text { 5'-GTCTTCCATGGTGTTATCAACCAC-3' }\end{array}$ \\
\hline $\begin{array}{l}\text { ASN1 promoter } \\
\text { G2-box mut }\end{array}$ & G2 mutation & $\begin{array}{l}\text { 5'-CTAGTCTAGAGCAGGCTCATTTCGCGGA } \\
\text { AAGTTG-3' } \\
\text { 5'-GTCTTCCATGGTGTTATCAACCAC-3' }\end{array}$ \\
\hline
\end{tabular}




\section{General Discussion}

\subsection{The G-box related element (GRE) and its cognate bZIP transcription factors constitute a novel regulatory unit which quantitatively modulates auxin-regulated transcription}

In the recent decades, tremendous progress has been made to unravel the molecular components and mechanisms that enable and control the co-ordinated manifestation of the manifold of auxin mediated plant processes. The vast majority of them has been ultimately ascribed to the encoded activity of auxin responsive genes which are, according to the current understanding, basically regulated by a complex network of members of the ARF- and AUX/IAA TF families (reviewed in Vanneste and Friml, 2009). Whereas the class of ARF-TFs has been found to be composed of both transcriptional activators and repressors, which actively bind AuxRE cis-elements in their auxin responsive target promoters, the AUX/IAA proteins exert their exclusively repressive function upon ARF-TF dimerisation (Abel and Theologis, 2010). Based on the ability of ARF and AUX/IAA proteins to form homo- and heterodimers between, or within the two TF classes, respectively and due to their individual spatio-temporal expression patterns, it has been postulated that the resulting intrinsic combinatorial capabilities enable a well-orchestrated implementation of the different auxin related developmental and environmental plant responses (Weijers et al., 2005).

Recent discoveries however indicated that the complexity of the auxin regulatory network is in fact larger and is not restricted to the well-established ARF- and AUX/IAA-TF families. For instance, it has only recently been demonstrated that also a member of the R2R3 class of MYB TFs plays a substantial role in auxin mediated transcription (Shin et al., 2007). In this respect it could be presented that AtMYB77 is able to directly bind the ARF-TF family specific C-terminus of the ARF7 protein and thereby contributes to the ARF-TF driven reporter gene expression, in a transient protoplast transfection system (Shin et al., 2007). The relevance of this synergistic action was further underpinned by gain- and loss-of function approaches in transgenic plants, which demonstrated a correlation between the level of MYB77 and the transcription of several auxin responsive genes and the manifestation of auxin-related root growth phenotypes (Shin et al., 2007). As in vitro studies additionally revealed that 
MYB77 is also able to bind further ARF-TFs it has been suggested that MYB77 and likely other homologous R2R3 MYB TFs might be of general importance in modulating auxin responsive gene expression (Shin et al., 2007). To validate this assumption by complementary approaches this work presents a genome-wide bioinformatic cis-element analysis of MYB- and ARF-TF related binding sites in the promoters of auxin responsive genes from A.thaliana and O.sativa (Chapter 1). In fact, within the set of all auxin inducible promoters from both evolutionary long separated species, an enrichment of composite modules of the typical AuxRE and the MYB-TF related binding site, MRE2 was demonstrated (Chapter 1). Besides this an especially high clustering of adjacent AuxRE and MRE cis-elements was detected in promoters from GH3 genes of several monocot and dicot plant species which were selected due to their homology to the well-characterised soybean $G H 3$ gene (Chapter 1). Two of these closely related GH3 genes, namely AtGH3.2 and AtGH3.3 were found to contain multiple MREs in their corresponding promoters (Chapter 1; Shin et al., 2007). To subsidiary address the significance of the MREs in the auxin mediated expression of the AtGH3.3 promoter, a mutational promoter analysis was conducted, in which the two existing MREs, located proximal to the transcriptional start site, (TSS) were mutated (Chapter 2). Consistent with the proposed mechanism of MYB-TFs to promote auxin mediated transcription via direct ARF-TF binding (Shin et al., 2007), a mutation of the AuxRE adjoining MRE motif led to a substantial decrease in auxin triggered expression, whereas a mutation of the AuxRE distal MRE resulted only in a minor, but still significant reduction (Chapter 2). This indicates that MYB-TFs primarily accomplish their quantitative modulating function upon binding of AuxRE close MRE coupling elements (Chapter 2), supporting the idea that specific MYB transcription factors might, in general synergistically promote auxin responsive transcription upon cooperative ARF-TF interaction (Shin et al., 2007).

As the expression of MYB77 was found to be affected by potassium deficient conditions, a role of MYB-TFs in modulating auxin responses according to limited nutrient supplies has been suggested (Shin et al., 2007). However a detailed analysis of the signal integration of the environmental aspects into auxin regulated transcriptional patterns, via these TF classes, remains elusive. Nevertheless these observations indicate that auxin responsive transcription is corporately regulated by auxin- and stress-related 
TF families and thus establishes a first model of combinatorial transcriptional control of auxin mediated responses.

Previous detailed analysis on the structural composition of the early auxin responsive soybean GH3 promoter (Liu et al., 1994, 1997; Ulmasov et al., 1995) revealed that the promoter's AuxRE motifs are frequently organised in repeats of composite modules consisting of AuxREs and the bZIP-TF related GREs. Complementary to this, work from our group demonstrated that the tobacco bZIPs, NtBZI-1 and NtBZI-2, which are known to form heterodimers, are able to induce the promoter of the soybean $G H 3$ homologous $N t G H 3$ gene. As moreover a down-regulation of NtBZI-2 or a constitutive expression of a truncated, likely dominant-negatively acting NtBZI-1 protein was found to result in the development of abnormal auxin related phenotypes, it has been hypothesised that bZIP-TFs might also play a yet undefined role in auxin mediated plant processes (Heinekamp et al., 2004; Iven et al., 2010). To address this issue, a genomewide bioinformatic cis-element analysis of auxin responsive promoters from Arabidopsis and rice was performed to examine the distribution and abundance of typical bZIP related binding sites (BREs) (Chapter 1). The results from this analysis clearly showed a significant enrichment of the GRE cis-element in the set of auxin inducible, but not repressible promoters from Arabidopsis and rice, compared to a randomized promoter dataset (Chapter 1). Similar to what had been observed for the association of MRE and AuxRE motifs also became apparent for the GRE and AuxRE cis-elements, which are in a modular fashion highly enriched in auxin-responsive promoters from both analysed species (Chapter 1). Despite the frequently observed physical proximity of the bZIP- and ARF-TF binding sites in many auxin responsive promoters, a direct interaction of bZIP and ARF proteins, as shown for MYB and ARFs (Shin et al., 2007) has not been detected by P2H and BiFC approaches, yet (CW, WDL unpublished data). However, it should be taken into account that only a small fraction of all possible combinations between members of the large ARF- and bZIP-TF classes were addressed. Moreover it should be considered that auxin responsive promoters are also enriched for tripartite modules, composed of GRE, AuxRE and MRE cis-elements (Chapter 1) and that interactions between bZIP- and MYB-TFs (Locatelli et al., 2000), as well as bZIP and ARF-like B3-type proteins, such as ABI3 (Lara et al., 2003; Alonso 
et al., 2009) were reported. Therefore direct or indirect interactions between proteins from these TF classes, which also might be associated in multi-protein complexes on auxin responsive promoters, cannot be excluded and might have been missed with the applied approaches.

Further examination of promoters from diverse auxin-related gene families, exhibited that the GRE-AuxRE cis-regulatory module was predominantly enriched in the promoters of the early auxin responsive Arabidopsis GH3 and AUX/IAA genes (Chapter 1), whereas the promoters of the commonly constitutively expressed ARFs (Ulmasov et al., 1999) did not show this module enrichment (Chapter 1). In order to determine if GRE motifs might be of general importance in the promoters of the group II, soybean homologous $G H 3$ genes, a comprehensive cis-element analysis of the corresponding GH3 promoters from several dicot and monocot plant species was performed (Chapter 1). Interestingly, the obtained results showed that the abundance of GRE motifs within the analysed GH3 promoters coincided with the homology of the promoter-associated GH3 genes to the soybean GH3. This observation suggests a combined conservation of the soybean GH3 protein function and its co-ordinated, presumably GRE motif dependent regulation within the group of highly $G m G H 3$ homologous genes (Chapter 1). To characterize the role of the GRE in auxin mediated transcription, mutational promoter cis-element analyses of the AtGH3.3 promoter were carried out (Chapter 2). Mutations in each of the four discernible, TSS close GRE motifs led to an approximately $20 \%$ reduction of the promoter's auxin inducibility, whereas mutations in all GREs further reduced it two merely $50 \%$ (Chapter 2). The quantitative nature of the GRE motif was further underpinned by the observation that the presence of a GRE ciselement significantly contributed to a promoter's auxin sensitivity, especially with respect to low auxin concentrations (Chapter 2). Considering that especially promoters of the early auxin responsive gene families such as $G H 3$ and $A U X / I A A s$ showed a significant enrichment of GRE-AuxRE bipartite modules (Chapter 1), it can be assumed that the early auxin responsive character of these genes might be at least partially attributed to the auxin sensitizing property of the GRE cis-element(s) in their promoters (Chapter 2). This assumption is supported by the observation that the artificial auxin responsive DR5 promoter, which lacks AuxRE coupling elements and functions by multimerisation of several AuxREs in a dense spacing (Hagen and Guilfoyle, 2002) 
shows a completely different auxin induction kinetic as e.g. the natural AtGH3.3 derived GRE-AuxRE (GRAUX) module (Chapter 2). In this respect the auxin sensitive GRAUX module exhibits its highest expression response to physiological low auxin levels (Chapter 2), whereas the artificial DR5 promoter shows a linear response to increasing auxin concentrations (Nakamura et al., 2003), indicative of a different type of registration for low auxin levels between the constructs. As this feature is likely mediated by the GRE motif, it underlines the relevance of this cis-element and its cognate TFs in enhancing auxin responsive transcription, particularly at low auxin concentrations.

In line with this, Heinekamp and co-workers (2004) identified that two tobacco bZIP transcription factors, namely NtBZI-1 and NtBZI-2 are capable to regulate the expression of the $\mathrm{NtGH3}$ gene in an auxin dependent manner. In order to further characterize bZIP action on the $G H 3$ promoter and to determine if this might be transferable to other auxin responsive genes, the activation properties of the closest Arabidopsis homologs were analysed with respect to the regulation of several auxinresponsive promoters, making use of transient protoplast transactivation assays (PTA) (Chapter 2). In Arabidopsis, the group $\mathrm{C}$ of AtbZIP-TFs, which is composed of AtbZIP9, -10, -25 and -63 and group S1, that encompasses AtbZIP1, -2, -11, -44 and 53 , have been described to be homologs of the tobacco NtBZI-1 or NtBZI-2 proteins, respectively (Strathman et al., 2001, Jakoby et al., 2002). Screening of the activation potential of these homologous AtbZIP-TFs revealed that especially the closely related group S1 AtbZIP2, -11 and -44 were able to induce the promoters of the AtGH3.3, AtAUX/IAA3, AtAUX/IAA7 and AtPIN4 genes (Chapter 2). However it should be mentioned that also group S1 AtbZIP53 and some group C AtbZIPs displayed some promoter specific induction capacity, such as AtbZIP10 on the AtPIN4 and AtbZIP63 on the AtGH3.3 promoter (Chapter 2). Most remarkable in this respect was the observation that the expression promoting group S1, as well as group C AtbZIPs strongly induced their auxin responsive target promoters already at the protoplasts' endogenous auxin levels. This strong induction could only moderately, with respect to the early auxin responsive AtGH3.3, or even hardly, concerning the weak auxin responsive AtAUX/IAA3, -7 and AtPIN4 promoters, further promoted by additional exogenous auxin application (Chapter 2). To determine if the bZIPs exert their 
activation potential upon direct promoter binding and via the typical bZIP related GRE cis-element, CHIP analysis and transient transactivation assays with GRE mutated promoter constructs were performed. By this means it was shown that AtbZIP11 and 44, which strongly enhanced the $A t G H 3.3$ promoter driven reporter gene expression, directly bound the GRE rich $A t G H 3.3$ promoter region and that the bZIPs mediated induction was dependent on functional GRE motifs (Chapter 2). In conclusion these observations further support the notion that bZIPs exert their activation capacity through direct GRE binding and thereby induce their auxin responsive target promoters already at low auxin concentrations (Chapter 2).

\subsection{AtbZIP11 related transcription factors interfere with the AUX/IAA - GH3 feed-back mechanism which modulates auxin mediated growth responses}

In order to confirm the relevance of bZIP-TFs on auxin mediated transcription on whole plant level and to address the influence of AtbZIP expression on auxin related phenotypic responses, stable transgenic gain- and loss-of function approaches for the closely related AtbZIP2, -11 and -44 AtbZIP-TFs, that most intensively and redundantly activated the analysed auxin responsive promoters in the transient PTA experiments, were established (Chapter 2). As neither T-DNA knock-out lines, nor constitutive overexpressors for these group S1 AtbZIPs could be obtained or exhibited an extremely dwarfed phenotype, respectively (Hanson et al., 2008; Weltmeier et al., 2009; Alonso et al., 2009), estradiol inducible amiRNA lines, which showed a simultaneous reduction of the closely related AtbZIP2, -11 and -44 transcripts and individual, inducible bZIP overexpressing lines were generated. A quantitative expression analysis of the auxin responsive AtGH3.3, AtAUX/IAA3, AtAUX/IAA7 and AtPIN4 genes in these transgenic plants confirmed that the full auxin mediated expression of the auxin responsive target genes is dependent on an adequate AtbZIP2, -11 and -44 expression and that it can be substantially enhanced by elevated bZIP induction (Chapter 2). Interestingly timecourse experiments further revealed individual AtbZIP-TF mediated induction kinetics for the analysed auxin responsive target genes. Whereas AtGH3.3 showed a fast induction response, briefly after the triggered expression of AtbZIP11 or AtbZIP44, the expression kinetics of the $A U X / I A A$ repressor genes were much slower and peaked at a 
time-point where AtGH3.3 expression was found to be down-regulated (Chapter 2). Additional studies on the regulation of the AtGH3.3 promoter in PTA experiments and in aux/iaa7 mutant plants, in fact exhibited a negative feed back regulation of the AUX/IAA3 and AUX/IAA7 repressor proteins on AtGH3.3 expression (Chapter 2).

These results suggest that in particular the group S1, AtbZIP11 related TFs are able to induce, with distinct induction kinetics, components of an auxin responsive, selfregulatory system. In this regulatory circuit the bZIPs rapidly induce the expression of the early auxin responsive AtGH3.3 gene and with slower kinetics also the AUX/IAA3 and -7 genes (Chapter 2). As the encoded activity of $A t G H 3.3$ has been implicated in regulating auxin homeostasis by conjugating the hormone to amino acids (Staswick et al., 2005) the enzyme reduces the cell's pool of bioactive auxin, which in turn leads to a stabilization of AUX/IAA repressor proteins (Gray et al., 2001). Consequently the bZIPs induced expression of $A U X / I A A 3$ and -7 represses under the emerging auxin deficient conditions AtGH3.3 transcription (Chapter 2), thereby balancing the bioactive auxin concentration at a new, lower threshold level. By this means AtbZIP-TFs might provide a self-adjusting system to rapidly fine-tune auxin responses by modulating the pool of active auxin and by inducing repressors of auxin-mediated transcription. However it has to be pointed out that additional experimental data is required to address the affect of the AtbZIP-TFs on cellular auxin concentration and protein stability of the involved components, to further characterise and validate this model (Figure 1).

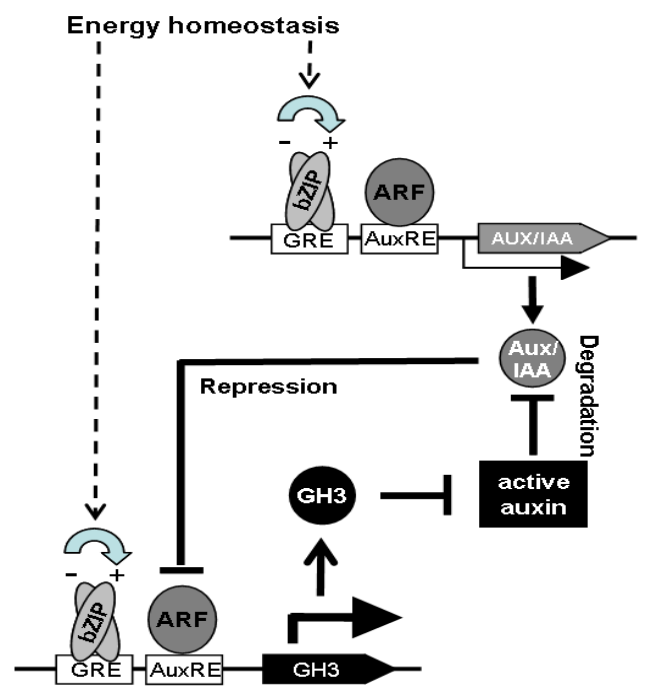

Figure 1: Model describing the function of group S1 bZIP factors in auxin-regulated transcription. A detailed description of the model can be found in the discussion in Chapter 2. 
As a bZIP mediated interference in auxin homeostasis and expression of AUX/IAA repressor proteins should lead to an impairment of auxin related responses, phenotypic alterations of bZIP over-expression plants were analysed. In this respect it was described that constitutive over-expression of specific group S1 AtbZIP-TFs in fact resulted in substantial growth retardation and thereby to dwarfed plant phenotypes (Hanson et al., 2008; Weltmeier et al., 2009; Alonso et al., 2009). In particular, the ectopic expression of AtbZIP2, -11 and -44 was demonstrated to produce severe dwarfed plants which hardly set any viable seeds (Hanson et al., 2008; Weltmeier et al., 2009), whereas expression of AtbZIP53 (Alonso et al., 2009) or AtbZIP1 (Dietrich et al., 2011) led to moderate or no apparent plant growth inhibitions, respectively. Interestingly, the capacity of each group S1 AtbZIP-TF to affect plant growth strongly correlated with their individual ability to induce the expression of the AtGH3.3 and AtAUX/IAA3 and -7 genes (Chapter 2).

In order to get a more detailed picture of the bZIP mediated growth inhibitory effects, inducible over-expressor lines of AtbZIP2, -11 and -44 were used and their bZIP associated plant growth phenotypes were assayed over a one week induction period (Chapter 2). Most remarkable in this respect was that the enhanced expression of each of these three bZIPs led at early induction time-points to obvious root growth alterations. For instance already after 36 hours of induced AtbZIP2, -11 or -44 expression, the majority of the transgenic plants revealed agravitropic root growth responses which, related to AtbZIP11 and -44 expressing plants, coincided with a significant decrease in the expression of a co-transfected auxin responsive DR5:GFP reporter construct in the plant's root tip (Chapter 2), indicative of alterations in the root's auxin signalling and/or auxin distribution. Only a few days later, additional and even more pronounced auxin related root growth phenotypes became apparent. Compared to the uninduced transgenic plants, the induced AtbZIP2, -11 and -44 overexpressing plant lines displayed a strong reduction of the primary root growth and of the auxin induced root hair formation, as well as an even more pronounced agravitropic root growth response (Chapter 2). In contrast to these auxin insensitive root growth phenotypes in the lower root parts, AtbZIP2, -11 and -44 expression led to an enhanced outgrowth of lateral roots in the more proximal root areas (Chapter 2). These opposing auxin related root growth responses along the primary root axis indicate that the 
observed phenotypes are at least partially caused by an abnormal auxin distribution. In fact, several publications indicated that the majority of the group S1 AtbZIP-TF mediated root growth phenotypes might be caused by the encoded activity of the auxin responsive bZIP target genes. For instance constitutive over-expression of the AtGH3.3 closest homologs (Chapter 2) such as AtGH3.5/WESl (Park et al., 2007), AtGH3.2/YDK1 (Takase et al., 2004) or AtGH3.6/DFL1 (Nakazawa et al., 2001) were found to result in severe dwarfed plants which, concerning the reduced root and shoot growth, also resembled the phenotypes of the gain-of function ataux/iaa3 (Tian et al., 2002; Tian and Reed, 1999; Weijers et al., 2005) and ataux/iaa7 mutants (Nagpal et al., 2000; Liscum and Reed, 2002; Wilson et al., 1990). In addition, these mutant plants, which express stabilized, auxin insensitive AtAUX/IAA3 and -7 proteins, that were postulated to be in part functionally redundant (Nagpal et al., 2000) showed a substantial agravitropic root growth response (Tian and Reed, 1999; Weijers et al., 2005; Wilson et al., 1990; Nagpal et al., 2000). Moreover, in particular the semidominant ataux/iaa7 mutants displayed also an inhibition of root hair formation (Wilson et al., 1990). Whereas the pleiotropic, auxin-insensitive growth phenotypes of the GH3 over-expressor plants were directly ascribed to the enzyme's activity in regulating auxin homeostasis via repressive auxin conjugation (Nakazawa et al., 2001; Takase et al., 2004; Park et al., 2007), it was postulated that both AUX/IAA proteins predominantly halt plant growth by repressing diverse auxin responsive genes which are involved in the coordinated manifestation of plant growth patterns (Tian et al., 2002; Nakamura et al., 2006). Recently published work from Scacchi and co-workers (2010) and Moubayidin and co-workers (2010) supported this assumption by shedding light on the mechanism of AtAUX/IAA3 mediated root growth regulation. They presented that the root localised expression of the auxin responsive AtAUX/IAA3 gene negatively regulated the expression of the main, basipetal auxin transport facilitators, namely AtPIN1, AtPIN3 and AtPIN7 and therefore controlled the polar auxin flow from the upper root parts to the root tip. Thus it was postulated that an enhanced expression of this factor leads to a significant decrease of the local auxin maximum in the meristematic root tip and thus to an imbalance in the local auxin/cytokinin ratio, which determines by a dynamic process of cell division and cell differentiation the apical root mersistem size and thus prospective root growth (Scacchi et al., 2010; Moubayidin et al., 2010). 
Therefore it is conceivable that the AtbZIP2, -11 and -44 promoted expression of the AtAUX/IAA3, but also of the AtGH3.3 and AtAUX/IAA7 genes might at least partially lead to the observed pleiotropic auxin insensitive root growth responses in the transgenic AtbZIP-TF over-expressing lines. However as already mentioned more detailed analysis on the AtbZIP induced changes in the root's auxin distribution are required, to further characterize the underlying mechanism which leads to the observed auxin insensitive phenotypes, but especially to the enhanced auxin controlled lateral root formation in the more upper root parts. Nevertheless it can be postulated that bZIP induced expression of the AtGH3 - AUX/IAA regulatory system provides means to rapidly modulate auxin mediated responses.

\subsection{AtbZIP11-related transcriptional activators modulate auxin- mediated gene expression by recruiting the histone acetylation machinery}

Only recently it was suggested that auxin responsive genes are regulated by an antagonistically acting system of histone acetylation and deacetylation (Long et al., 2006; Szemenyei et al., 2008). In this respect it was demonstrated, that the repressive function of the AUX/IAA-TF, IAA12 on the expression of auxin responsive genes is dependent on its interaction with members of the so-called TOPLESS (TPL) corepressor protein family (Szemenyei et al., 2008). As a positive genetic interaction between TPL1 and the Histone ㅁe-Acetylase 19 (HDA19) was identified and the

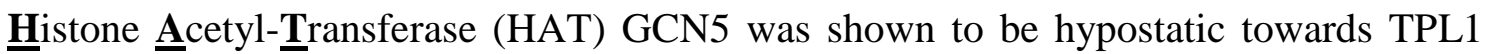
mediated responses, it was suggested that the AUX/IAA repressor proteins as well as TPL1 and HDA19 are located in a common repressive signalling pathway, whereas the HAT GCN5 is positioned in a counteracting, expression promoting system. By this means the respective proteins might provide a dynamic regulatory mechanism to rapidly adjust auxin responsive gene expression (Long et al., 2006; Szemenyei et al., 2008).

In order to verify this hypothesis and to address if the bZIP induced auxin responsive genes are regulated by histone acetylation or deacetylation respectively, a GCN5 family specific HAT- or a broad-spectrum HDAC inhibitor was applied (Chapter 3). In fact the results from this pharmacological approaches, revealed that the auxin-induced expression of the group S1 AtbZIP target genes; AtGH3.3, AtAUX/IAA3, AtAUX/IAA7 
and AtPIN4 was indeed dependent on a functional GCN5 specific histone acetylation system and was controlled by a counteracting deacetylation machinery (Chapter 3).

The HAT GCN5 was demonstrated to be a conserved co-activator of bZIP-TFs in yeast and plants (Topalidou et al., 2003; Locatelli et al., 2009). In order to determine if the bZIP-TF mediated induction of auxin-responsive target genes is at least partially attributed to the recruitment of a histone remodelling complex, further reverse genetic and biochemical approaches were conducted (Chapter 3). By this means it could be demonstrated that mutants of diverse HAT genes, including the gcn5 and the GCN5 related hag4 and hag5 mutants exhibited a significant, in part HAT specific reduction of the bZIP auxin-responsive target genes (Chapter 3). According to the concept of HAT regulated auxin responses, mutations in these genes should consequently result in auxin insensitive phenotypes. In fact, it was presented that gcn5 mutant plants display pleiotropic auxin-related phenotypic alterations such as severe dwarfism, loss of apical dominance, aberrant meristem function, abnormal root and leaf development and reduced petal and stamen growth (Vlachonasios et al., 2003, Bertrand et al., 2003; Long et al., 2006; Kornet and Scheres, 2009). Similar observations were reported for mutants of the highly homologous, apparently functional redundant HAG4 and HAG5 genes. Whereas homozygous double mutants were not viable, sesqui-mutant plants (HAG4/hag4 - hag5/hag5) displayed severe disorders in the auxin dependent pollen development (Latrasse et al., 2008; Cecchetti et al., 2008; Iven et al., 2010).

However all these HAGs seem to be involved in modulating auxin-regulated transcription, the observed differences in the HAG mutant phenotypes and their individual ability to affect auxin-regulated gene expression (Chapter 3) indicate that the GCN5 enzyme and the two HATs of the related MYST gene family (HAG4 and HAG5) might implement in part distinct auxin-mediated responses.

The HAT GCN5 was described to be in general associated in large protein complexes. Similar to the yeast GCN5, the Arabidopsis and maize homologous enzymes are incorporated in a SAGA-like histone remodelling complex (Vlachonasios et al., 2003; Bhat et al., 2004).

In maize the bZIP-TF O2 was demonstrated to regulate seed storage genes by recruiting the SAGA complex to its target promoters via a direct interaction with the complex adapter protein ADA2 (Locatelli et al., 2009; Bhat et al., 2004) 
Analogously, it was tested if group S1 and C AtbZIPs might also be able to recruit this complex upon binding to the ZmADA2 homologous Arabidopsis AtADA2a and AtADA2b complex components. In fact, particularly the group S1 AtbZIP2, -11 and -44 TFs exhibited a strong capacity to bind both AtADA2 adapter proteins, whereas the $\mathrm{O} 2$ orthologues AtbZIP10 and AtbZIP25 (Alonso et al., 2009; Jakoby et al., 2002) showed a significant binding exclusively to AtADA2b (Chapter 3). This indicates that diverse bZIP-TFs are in principle able to recruit the SAGA complex to regulate their diverse target genes. However further work is necessary to validate this assumption and to define the individually involved SAGA complex HAT and adapter components, which besides the promoter bound TFs apparently also confer some specificity to the regulatory system.

Based on the acidic nature of the N-terminus of the group S1 AtbZIP-TFs (Chapter 3), and the fact that a similar structured region was already suggested to be the potential ADA2 interaction surface within the $\mathrm{O} 2$ protein (Bhat et al., 2004), the activation and ADA2 binding properties of N-terminally truncated AtbZIP11 and AtbZIP44 derivatives were analysed in transient protoplast transfection assays and in stable transgenic plants. The obtained results demonstrated that ADA2 binding was mediated by the bZIP's N-terminus, which acted as an activation domain and was crucial to induce the expression of the bZIP auxin responsive target genes and for the manifestation of bZIP mediated auxin-related plant growth responses (Chapter 3 ).

As variants of the observed polar, acidic activation and ADA2 binding domain are apparently conserved within certain homologous bZIP-TF classes from diverse species, these results could explain the reported, in part dominant negative property of the Arabidopsis group C and maize O2 homologous tobacco NtBZI-1 protein on the expression of the auxin responsive $N t G H 3$ gene and on auxin mediated plant responses (Heinekamp et al., 2004).

In order to finally clarify, if the activation potential of the AtbZIP11 and -44 TFs was mediated by the recruitment of a chromatin remodelling, GCN5 histone acetylation complex to the auxin responsive target promoters, CHIP analyses were performed (Chapter 3). By this means it could be demonstrated that upon enhanced AtbZIP11 or 44 promoter binding, the rate of GCN5 specific histone acetylation in the GRE rich AtGH3.3 promoter region and the assembly of the RNA polymerase II near the 
transcriptional start site of the GH3.3 gene was significantly increased (Chapter 3). As this went along with a bZIP mediated enhanced transcription of the AtGH3.3 gene it can be assumed that in particular the AtbZIP11-related TFs are capable to induce their auxin responsive target genes, by consulting an Arabidopsis SAGA-like HAT complex to their target promoters and thereby represent a counteracting system to the repressive action of the AUX/IAA-TFs, which are thought to control target gene expression by histone deacetylation. As the cis-regulatory GRE-AuxRE module was found to be frequently distributed in the promoters of auxin responsive genes (Chapter 1) the bZIP/GRE regulatory system likely constitutes an expression modulating system, which enables a rapid and highly dynamic regulation of the bZIP auxin responsive target genes and their associated responses (Figure 2).

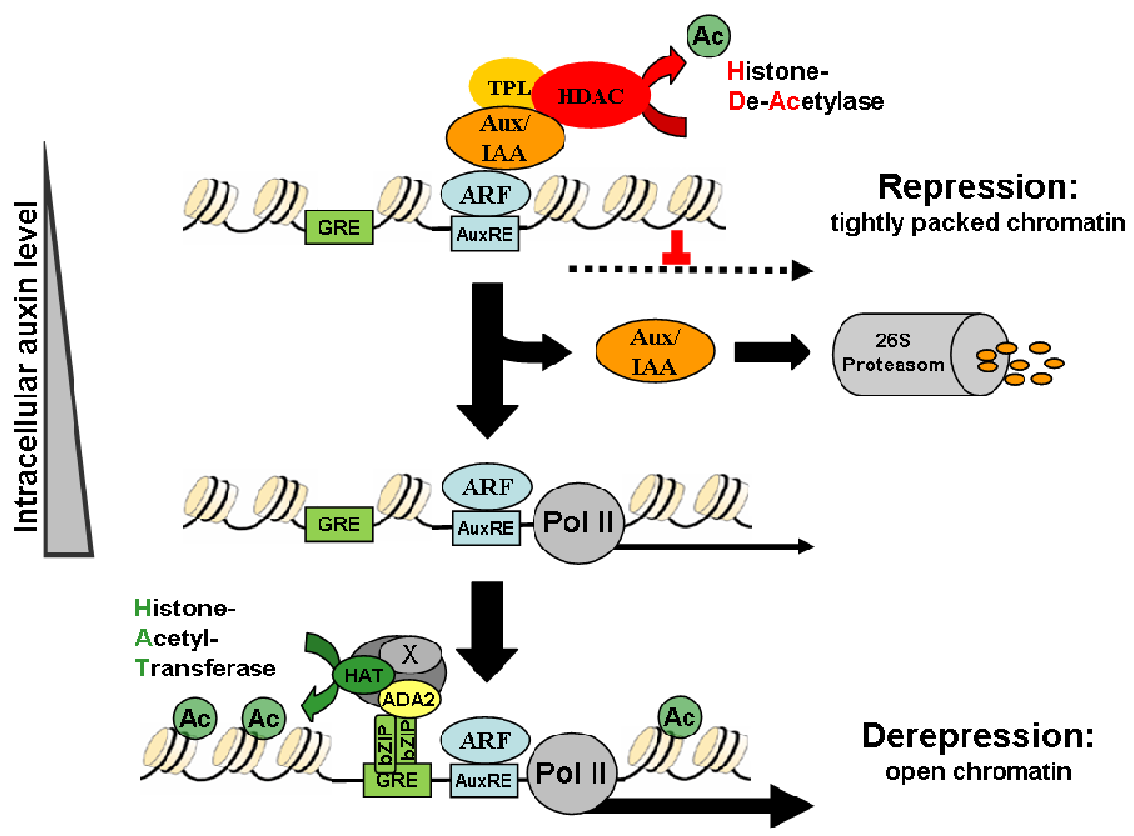

Figure 2: BZIP transcription factors recruit the Arabidopsis SAGA complex to their auxin responsive target genes. A detailed description of the model can be found in the discussion in Chapter 3 .

In this work it was demonstrated that group S1 AtbZIP11 related TFs are able to recruit a GCN5/ADA2 composed histone-remodelling complex to their auxin responsive genes, to induce their expression. However besides these TFs also further group S1 and C AtbZIPs (Chapter3) and also group D AtbZIP-TFs (unpublished results) are able to bind the adapter protein AtADA2b, in in vivo approaches. Therefore it is conceivable that these and likely other bZIP-TFs, which exhibit a polar, acidic ADA2 interaction 
domain, recruit the SAGA complex to their target promoters and that this might constitute a general regulatory mechanism by which bZIPs are able to rapidly and dynamically regulate their target genes. However prospective work is required to verify this assumption and to define the relevance of the SAGA complex recruitment in diverse bZIP controlled responses.

\subsection{BZIP factors of the C/S1 network are proposed to balance plant growth responses and adaptive metabolic reprogramming under energy deprived conditions}

In this work it was presented that members of the AtbZIP-TFs are able to modulate auxin responses by interfering with auxin mediated transcription. However a characterisation of the biological relevance of this regulation remains elusive. Nevertheless analysis of AtbZIP expression profiles and further published results indicate that they are involved in adapting auxin-related growth responses to internal and external stimuli.

Reffering to this it was shown that the endogenous expression of all group S1 AtbZIPTFs is regulated on transcriptional and post-transcriptional level by the current sucrose concentration within the plant (Weltmeier et al., 2009; Smeekens et al., 2010; Dietrich et al., 2011), suggesting that the expression of these growth modulating TFs (Chapter 2; Hanson et al., 2008; Weltmeier et al., 2009; Alonso et al., 2009) is interlinked with the plants prevailing energy status. More precisely, it was revealed that the expression of AtbZIP11 and to a minor extent AtbZIP44 is induced by sucrose, whereas AtbZIP1 and 53 are effectively expressed under energy deprived conditions (Dietrich et al., 2011; Chapter 4). Despite their opposing sucrose dependent induction patterns it could be demonstrated that the translation of all group S1 mRNAs is effectively regulated by an uORF encoded signalling peptide, which enables repression of translation in a sucrose concentration dependent manner (Wiese et al., 2004; Hummel et al., 2009; Rahmani et al., 2009). This suggests that group S1 bZIPs primarily exert their function under energy deprived conditions, when sucrose levels are low and currently synthesized or existing, presumably primed pools of bZIP transcripts can be effectively translated. Based on the observed growth inhibitory activity of group S1 bZIPs and their coordinated expression during energy starvation, it can be hypothesized that these bZIP-TFs represent 
important energy signal integrators in auxin mediated transcription, to adjust plant growth to the prevailing energy supplies. This assumption is further supported by the observation that the expression of group S1 bZIPs is, depending on the individual bZIPgene, differently well-pronounced regulated in a diurnal manner (Figure 3A, B). This diurnal regulation of group S1 AtbZIP-TFs is presumably conferred by the circadian clock, as it was shown that the integral clock component CCA1 directly binds the AtbZIP1 promoter (Gutièrrez et al., 2008) and/or might be mediated by changes in the plant's sucrose concentration. This sugar concentration is directly linked to the prevailing photosynthesis rates and thus continuously rises during the day and declines during the night (Sulpice et al., 2009), thereby presumably dynamically regulating group S1 bZIP expression via the uORF encoded sucrose control-peptide. As further analysis of the expression profiles of the AtbZIP regulated, growth controlling AtGH3.3 - AtAUX/IAA3/7 system, also demonstrated that all components are in fact preferably expressed during the night, when carbon supplies are limited, it can certainly be suggested that the enhanced expression of especially AtbZIP11 during the night induces this growth-regulating system to dynamically adjust plant growth under energy deprived conditions (Figure 3A).

Besides their envisaged role in adaptive growth regulation by interfering with the expression of diverse auxin responsive genes, specific group S1 AtbZIP-TFs were found to additionally regulate several key enzymes of the plant's primary metabolism and thereby implement a metabolic reprogramming to counteract energy starvation during the night (Chapter 4; Smeekens et al., 2010; Dietrich et al., 2011). In particular AtbZIP1 and AtbZIP53 (Weltmeier et al., 2006; Dietrich et al., 2011) but also AtbZIP2 and -11 (Hanson et al., 2008; Baena-Gonzalez et al., 2007) were described to modulate amino-acid metabolism by for instance enhancing the expression of the dark-induced asparagine synthase 1 (ASN1) gene, which catalyses the last step of the glutamate dependent asparagine (Asn) synthesis. As asparagine contains less carbon that glutamine, but the same amount of nitrogen it is preferentially used as nitrogen transport form, under carbon limited conditions (Lam et al., 1994). Furthermore Dietrich and coworkers (2011; Chapter 4) demonstrated, that besides the ASN1 gene also a great part of the upstream Asn biosynthesis genes are induced by AtbZIP1 and AtbZIP53 and that these AtbZIPs additionally regulate genes of the proline- as well as the branched chain 
amino acid catabolic pathways during energy deprived conditions. Complementary to this, it was shown that also AtbZIP2 (Baena-Gonzalez et al., 2007) and AtbZIP11 (Hanson et al., 2008) were involved in this process by regulating the ASN1, and referred to AtbZIP11 also the proline dehydrogenase 2 (ProDH2) gene (Hanson et al., 2008). An expression profile analysis of the involved AtbZIP1, -2, -11 and -53 TF genes and their targets, such as ASN1 and ProDHI once again showed that all respective genes exhibited a diurnal expression rhythm (Figure 3B).

Altogether, it can therefore be hypothesized that specific sets of group S1 and likely also their group $\mathrm{C}$ heterodimerisation partners are involved in diverse regulatory networks to adjust plant growth and metabolism to the fluctuating energy supplies between day and night or presumably also during further energy demanding stress conditions (Figure 4). As the expression and activity of group S1 AtbZIP-TFs was shown to be regulated by several post-transcriptional and -translational events, further investigation of AtbZIP protein levels and for instance protein phosphorylation are required to unravel the underlying mechanism of bZIP action to co-ordinate plant growth.
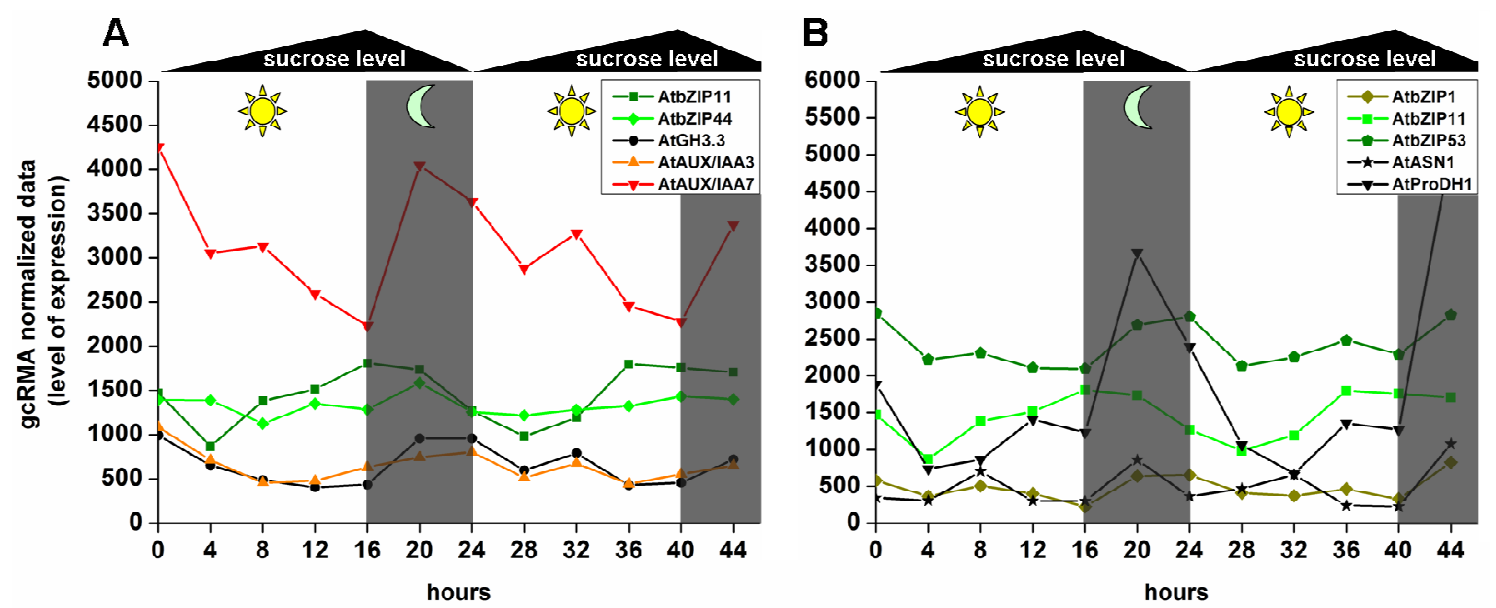

Figure 3: Expression profiles of group S1 AtbZIP-TFs and their putative target genes calculated from microarray data described by Michael and co-workers (2008). Due to the plant's photosynthetic activity, sucrose concentration rises during the day and declines at night (Sulpice et al., 2009) A) AtbZIP11 and AtbZIP44 expression is induced by sucrose, whereas AtbZIP1 and AtbZIP53 expression is induced by energy deprivation (Dietrich et al., 2011). Translation of the newly synthesized group S1 AtbZIP1 and -53 transcripts or the likely during the day accumulated transcript pools of AtbZIP11 and -44 is repressed in a sucrose concentration dependent manner by the so-called SIRT mechanism (Rahmani et al., 2009). Therefore, translation of group S1 AtbZIPs is supposed to occur during the night, when sucrose concentration declines. As translation of the group S1 AtbZIPs is directly linked to sucrose availability their target genes which have been implicated in (A) repressing plant growth or $(\mathbf{B})$ implementing adaptive metabolic reprogramming, enable adaptive plant responses to manage energy resources during energy deprived conditions. 
Due to the limited quantity of energy resources plants have to co-ordinately manage their allocation according to the current energy supplies and demands. During stressful environmental conditions such as cold, drought or pathogen attack, it is therefore not surprising that plants generally react with a decrease in their energy consuming growth processes to presumably save resources which are required for the massive adaptive metabolic reprogramming which is mandatory to counteract the prevailing stress conditions (Park et al., 2007; Burdon et al., 2003; Heil, 2002; Rymen et al., 2007). As most growth related processes are controlled by auxin mediated gene expression it was postulated that these diverse environmental signals are presumably integrated in the auxin signalling pathway (Shin et al., 2007; Park et al., 2007). Indeed, in response to pathogen challenge it was observed that an effective plant defence response against pathogenic encounters is frequently associated with an impairment of auxin signalling and expression of several auxin responsive genes which is accompanied by reduced plant growth (Navarro et al., 2006; Wang et al., 2007). In contrast to this, it was found that successful pathogens have evolved various strategies to counteract this plant triggered impairment of auxin mediated responses by for instance producing large amounts of auxin (Glickmann et al., 1998) or titrating AUX/IAA repressors of auxin responsive gene expression (Padmanabhan et al., 2006, 2008). Consistently, exogenous auxin application was found to result in an increase in pathogen virulence, indicating that auxin can act as a susceptibility factor (Navarro et al., 2006; Wang et al., 2007).

In general diverse plant defence responses towards biotrophic pathogens are mediated by the signalling molecule SA and its downstream signalling components NPR1 and specific group D bZIP-TFs (Pieterse et al., 2009; Spoel and Dong, 2008). Recent studies discovered that SA mediated processes negatively affect auxin signalling, which suggests that plants might adapt their auxin mediated responses during pathogen challenge by a repressive action of the pathogen inducible SA pathway. In this respect it was demonstrated that exogenous SA application as well as pathogen infection led, in a NPR1 dependent manner, to a down-regulation of a largely concurrent set of auxin responsive and auxin signalling genes, whereas at least two $G H 3$ genes were found to be up-regulated (Wang et al., 2007). Moreover it was shown that several SA accumulating mutants such as sncl, cpr6 or cpr5 exhibit diverse growth alterations which are reminiscent of auxin deficiency (Wang et al., 2007; Bowling et al., 1997; 
Clarke et al., 1998; Li et al., 2001). Quantitative auxin measurements in fact revealed that the free auxin levels were significantly decreased in the SA over-producing sncl and cpr6 mutant plants (Wang et al., 2007). A possible explanation for this SA mediated reduction of the free auxin levels might be at least partially attributed to the enhanced SA inducible expression of the AtGH3.3 and its closely related AtGH3.5 gene (Wang et al., 2007), which encoded activities have been described to be involved in repressive auxin conjugation to diverse amino acids, thereby controlling auxin homeostasis (Staswick et al., 2005). In this work it was presented that the promoters of the homologous group II GH3 genes from several plant species show a conserved enrichment for the SA responsive TGACG motifs (Chapter 1) which was found to be a typical binding site of group D bZIP-TFs (Jakoby et al., 2002; Butterbrodt et al., 2006). Indeed, group D bZIPs, which are also known as TGA-TFs are able to induce in particular pathogenesis related (PR) genes in a NPR1 and SA dependent manner upon TGACG cis-element binding (Pape et al., 2010a , b). In transient protoplast transfection assays TGA-TF were also able to quite redundantly induce the AtGH3.3 promoter (unpublished results). Moreover they were able to bind the AtADA2b protein (unpublished results), which is the postulated adapter component of an Arabidopsis SAGA-like HAT complex (Vlachonasios et al., 2003; Locatelli et al., 2009). As the TGA-TF target gene $P R 1$ is activated by TGAs and its promoter is histone 3 hyperacetylated after SA and TGA induction (Butterbrodt et al., 2006; Koornneef et al., 2008) it is tempting to speculate that group D AtbZIP-TF might play a dual role in plant defence responses by dynamically inducing $P R$ genes for direct pathogen defence and GH3 genes for adaptive auxin conjugation and growth regulation to release energy supplies to balance the fitness costs of effective pathogen defence (Figure 4). However, further work is needed to characterise the role of TGA-TFs in this antagonistic SAauxin crosstalk and to address the issue if TGA-TF mediated $P R I$ and $G H 3$ gene induction could also be exerted by recruiting HAT complexes to their auxin- and pathogenesis-related target genes. 
In conclusion these examples suggest that in particular group S1 AtbZIP-TFs but likely also further bZIP-TFs constitute a regulatory system to fine-tune auxin-mediated responses with respect to internal and external stimuli. By this means AtbZIP-TFs could assist to manage the plant's energy resources according to the prevailing energy demands to sustain optimal plant growth and survival.

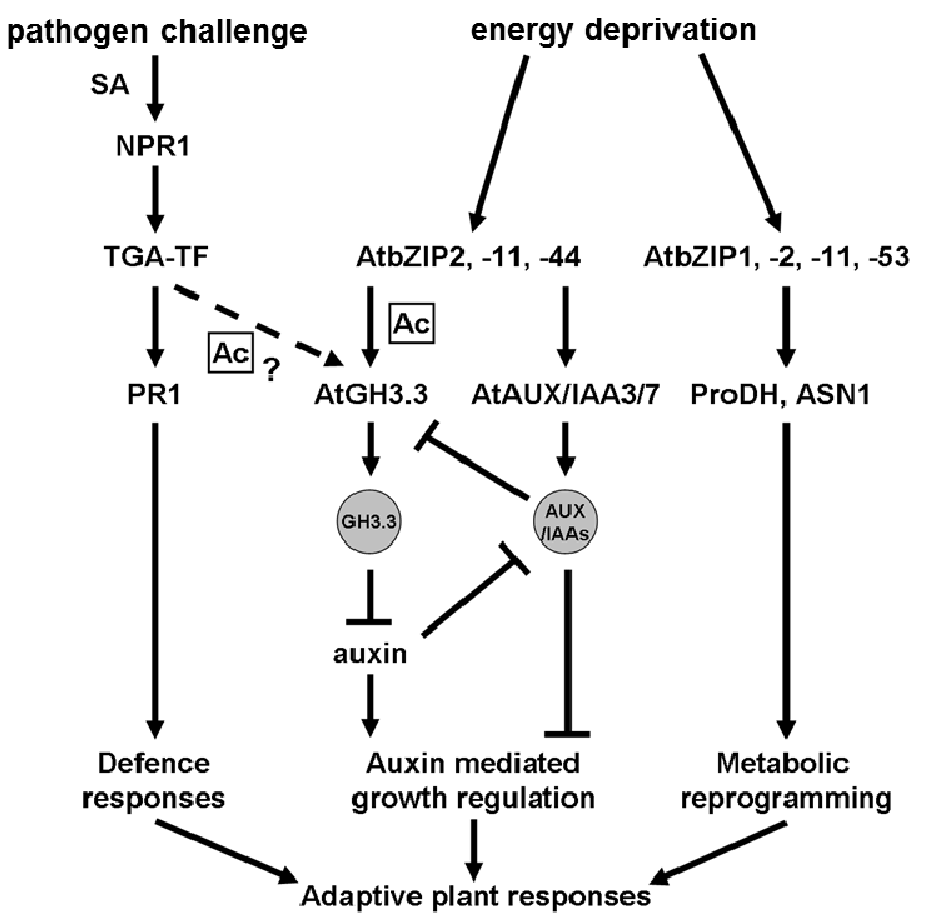

Figure 4: Proposed model of AtbZIP-TF action in adaptive plant processes under energy demanding conditions. Upon pathogen challenge, which elicits a SA accumulation, group D AtbZIP-TFs (TGAs) are trans-activated by NPR1 interaction and induce pathogenesis-related (PR) genes as well as presumably the auxin responsive $A t G H 3.3$ gene, to repress auxin mediated growth responses in order to release energy supplies which are required to balance the fitness costs of effective pathogen defence. Under energy deprived conditions specific group S1 AtbZIP-TFs induce genes of the amino acid metabolism and presumably also the auxin responsive AtGH3.3-AtAUX/IAA3/7 growth regulatory system to adapt plant metabolism and growth to the prevailing energy status of the plant. Envisaged gene activation by bZIP mediated histone acetylation is labelled with AC. 


\section{$5 \quad$ Appendix}

\subsection{Abbreviations}

\section{General abbreviations}

\begin{tabular}{|c|c|}
\hline AA & Amino acid \\
\hline $\mathrm{ABA}$ & Abscisic acid \\
\hline $\mathrm{ABI} 3$ & ABA INSENSITIVE 3 gene \\
\hline ABP1 & Auxin binding protein 1 \\
\hline $\mathrm{AD}$ & Activation domain \\
\hline AFB & Auxin signalling F-Box protein \\
\hline amiRNA & Artificial micro RNA \\
\hline ANOVA & Analysis of variance \\
\hline $\mathrm{ARF}$ & Auxin response factor \\
\hline ASN1 & Asparagine synthase 1 gene \\
\hline At & Arabidopsis thaliana \\
\hline AtbZIP & Arabidopsis thaliana basic leucine zipper \\
\hline AuxRE & Auxin responsive element \\
\hline $\mathrm{BD}$ & Binding domain \\
\hline bps & Base pairs \\
\hline BRE & BZIP related element \\
\hline ChIP & Chromatin Immunoprecipitation \\
\hline CoIP & Co-Immunoprecipitation \\
\hline Col-0 & Columbia-0 (Arabidopsis ecotype) \\
\hline CRES-T & Chimeric repressor silencing technology \\
\hline DMSO & Dimethylsulfoxide \\
\hline DNA & Deoxyribonucleic acid \\
\hline dNTPs & Deoxynucleotide Triphosphate \\
\hline e.g. & For example \\
\hline
\end{tabular}

EAR-domain ERF associated amphiphilic repressor domain

Est 17-ß-estradiol 


\begin{tabular}{|c|c|}
\hline Et al. & Et alia / and others \\
\hline GCN5 & General Control Nondepressible 5 \\
\hline $\mathrm{Gm}$ & Glycine max \\
\hline GNAT & GCN5 related N-acetyltransferase \\
\hline GRAUX & GRE-AuxRE module \\
\hline GRE & G-box related element \\
\hline GUS & ß-glucuronidase \\
\hline $\mathrm{H}$ & Hour \\
\hline $\mathrm{H} 3$ & Histone 3 \\
\hline HA & Human influenza hemagglutinin peptide \\
\hline HAG & Histone acetyltransferase of the GNAT/MYST superfamily \\
\hline HAT & Histone acetyltransferase \\
\hline HDAC & Histone deacetylase \\
\hline IAA & Indole-3-acetic acid \\
\hline IAM & Indole-3-acetamide \\
\hline IAOx & Indole-3-acetaldoxime \\
\hline IGP & Indole-3-glycerol-phosphate \\
\hline IPA & Indole-3-pyruvic acid \\
\hline $\mathrm{kDA}$ & Kilo Dalton \\
\hline ko & Knock out \\
\hline M & Molar \\
\hline MAPK & Mitogen-activating protein kinase \\
\hline MRE & Myb related element \\
\hline mRNA & Messenger ribonucleic acid \\
\hline NAA & 1-Naphthaleneacetic acid \\
\hline NAN & Neuraminidase gene \\
\hline $\mathrm{Nt}$ & Nicotiana tabacum \\
\hline O.sativa & Oryza sativa \\
\hline $\mathrm{O} 2$ & Opaque-2 \\
\hline OEX & Overexpessor \\
\hline $\mathrm{P}$ & Probability \\
\hline $\mathrm{P} 2 \mathrm{H}$ & Protoplast-2-hybrid assay \\
\hline
\end{tabular}


PBS Phosphate buffered saline

PCR Polymerase chain reaction

PIN PIN formed mutant phenotype

Pro promoter

ProDH Prolin Dehydrogenase

PTA Protoplast transactivation assays

qRT-PCR Quantitative realtime PCR

RNPII RNA Polymerase II

rpm Revolutions per minute

SA Salicylic acid

SAGA Spt-Ada-Gcn5-Acetyltransferase

SCF Skp-cullin-F-box complex

SD Standard deviation

SDS-PAGE Sodium dodecyl sulphate - poly acrylamide gel electrophoresis

SEM Standard error median

SIRT Sucrose induced repression of translation

SnRK1 SNF1 related kinase 1

TAA Tryptophan aminotransferase of Arabidopsis

TAM Tryptamine

T-DNA Transfer DNA

TF Transcription factor

TIR1 TRANSPORT INHIBITOR RESPONSE 1 gene

TPL TOPLESS gene

TSA Trichostatin A

TSS Transcriptional start site

UBQ5 Ubiquitin 5 gene

uORF Upstream open reading frame

UTR Untranslated region

WT Wildtype

XVE Estradiol inducible espression system 


\section{Metric prefixes}

$\begin{array}{lll}\mathrm{k} & \text { kilo } & \left(10^{3}\right) \\ \mathrm{m} & \text { milli } & \left(10^{-3}\right) \\ \mu & \text { mikro } & \left(10^{-6}\right) \\ \mathrm{n} & \text { nano } & \left(10^{-9}\right) \\ \mathrm{p} & \text { pico } & \left(10^{-12}\right)\end{array}$

\section{Amino acid abbreviations}

$\begin{array}{lll}\text { A } & \text { Ala } & \text { Alanine } \\ \text { C } & \text { Cys } & \text { Cysteine } \\ \text { D } & \text { Asp } & \text { Aspartic acid } \\ \text { E } & \text { Glu } & \text { Glutamic acid } \\ \text { F } & \text { Phe } & \text { Phenylalanine } \\ \text { G } & \text { Gly } & \text { Glycine } \\ \text { H } & \text { His } & \text { Histidine } \\ \text { I } & \text { Ile } & \text { Isoleucine } \\ \text { K } & \text { Lys } & \text { Lysine } \\ \text { L } & \text { Leu } & \text { Leucine } \\ \text { M } & \text { Met } & \text { Methionine } \\ \text { N } & \text { Asn } & \text { Asparagine } \\ \text { P } & \text { Pro } & \text { Proline } \\ \text { Q } & \text { Gln } & \text { Glutamine } \\ \text { R } & \text { Arg } & \text { Arginine } \\ \text { S } & \text { Ser } & \text { Serine } \\ \text { T } & \text { Thr } & \text { Threonine } \\ \text { V } & \text { Val } & \text { Valine } \\ \text { W } & \text { Trp } & \text { Tryptophan } \\ \text { Y } & \text { Tyr } & \text { Tyrosine }\end{array}$

\section{Nucleotide abbreviations}
A
Adenine
C
Cytosine
G
Guanosine
$\mathrm{T}$
Thymine
$\mathrm{U}$
Uracile 


\section{References}

Abel S, Nguyen MD, Theologis A. 1995. The PS-IAA4/5-like family of early auxin-inducible mRNAs in Arabidopsis thaliana. J Mol Biol, 251: 533.

Abel S, Oeller PW, Theologis A. 1994. Early auxin-induced genes encode short-lived nuclear proteins. Proc Natl Acad Sci U S A, 91: 326.

Abel S, Theologis A. 2010. Odyssey of auxin. Cold Spring Harb Perspect Biol, 2: a004572.

Acharya A, Ruvinov SB, Gal J, Moll JR, Vinson C. 2002. A heterodimerizing leucine zipper coiled coil system for examining the specificity of a position interactions: amino acids I, V, L, N, A, and K. Biochemistry, 41: 14122.

Alonso R, Onate-Sanchez L, Weltmeier F, Ehlert A, Diaz I, Dietrich K, Vicente-Carbajosa J, Droge-Laser W. 2009. A pivotal role of the basic leucine zipper transcription factor bZIP53 in the regulation of Arabidopsis seed maturation gene expression based on heterodimerization and protein complex formation. Plant Cell, 21: 1747.

Anzola JM, Sieberer T, Ortbauer M, Butt H, Korbei B, Weinhofer I, Mullner AE, Luschnig C. 2010. Putative Arabidopsis transcriptional adaptor protein (PROPORZ1) is required to modulate histone acetylation in response to auxin. Proc Natl Acad Sci U S A, 107: 10308.

Baena-Gonzalez E, Rolland F, Thevelein JM, Sheen J. 2007. A central integrator of transcription networks in plant stress and energy signalling. Nature, 448: 938.

Bajguz A, Piotrowska A. 2009. Conjugates of auxin and cytokinin. Phytochemistry, 70: 957.

Baxevanis AD, Vinson CR. 1993. Interactions of coiled coils in transcription factors: where is the specificity? Curr Opin Genet Dev, 3: 278.

Benkova E, Michniewicz M, Sauer M, Teichmann T, Seifertova D, Jurgens G, Friml J. 2003. Local, efflux-dependent auxin gradients as a common module for plant organ formation. Cell, 115: 591.

Bennett MJ, Marchant A, Green HG, May ST, Ward SP, Millner PA, Walker AR, Schulz B, Feldmann KA. 1996. Arabidopsis AUX1 gene: a permease-like regulator of root gravitropism. Science, 273: 948 .

Bertrand C, Bergounioux C, Domenichini S, Delarue M, Zhou DX. 2003. Arabidopsis histone acetyltransferase AtGCN5 regulates the floral meristem activity through the WUSCHEL/AGAMOUS pathway. J Biol Chem, 278: 28246.

Bhat RA, Borst JW, Riehl M, Thompson RD. 2004. Interaction of maize Opaque-2 and the transcriptional co-activators GCN5 and ADA2, in the modulation of transcriptional activity. Plant Mol Biol, 55: 239.

Blilou I, Xu J, Wildwater M, Willemsen V, Paponov I, Friml J, Heidstra R, Aida M, Palme K, Scheres B. 2005. The PIN auxin efflux facilitator network controls growth and patterning in Arabidopsis roots. Nature, 433: 39.

Bowling SA, Clarke JD, Liu Y, Klessig DF, Dong X. 1997. The cpr5 mutant of Arabidopsis expresses both NPR1-dependent and NPR1-independent resistance. Plant Cell, 9: 1573.

Braun N, Wyrzykowska J, Muller P, David K, Couch D, Perrot-Rechenmann C, Fleming AJ. 2008. Conditional repression of AUXIN BINDING PROTEIN1 reveals that it coordinates cell division and cell expansion during postembryonic shoot development in Arabidopsis and tobacco. Plant Cell, 20: 2746.

Burdon JJ, Thrall PH. 2003. The fitness costs to plants of resistance to pathogens. Genome Biol, 4: 227.

Butterbrodt T, Thurow C, Gatz C. 2006. Chromatin immunoprecipitation analysis of the tobacco PR1a- and the truncated CaMV $35 \mathrm{~S}$ promoter reveals differences in salicylic acid-dependent TGA factor binding and histone acetylation. Plant Mol Biol, 61: 665.

Cecchetti V, Altamura MM, Falasca G, Costantino P, Cardarelli M. 2008. Auxin regulates Arabidopsis anther dehiscence, pollen maturation, and filament elongation. Plant Cell, 20: 1760.

Chandler JW. 2009. Local auxin production: a small contribution to a big field. Bioessays, 31: 60. 
Chen JG, Shimomura S, Sitbon F, Sandberg G, Jones AM. 2001. The role of auxin-binding protein 1 in the expansion of tobacco leaf cells. Plant J, 28: 607.

Cheng Y, Dai X, Zhao Y. 2006. Auxin biosynthesis by the YUCCA flavin monooxygenases controls the formation of floral organs and vascular tissues in Arabidopsis. Genes Dev, 20: 1790.

Cheng Y, Dai X, Zhao Y. 2007. Auxin synthesized by the YUCCA flavin monooxygenases is essential for embryogenesis and leaf formation in Arabidopsis. Plant Cell, 19: 2430.

Choi H, Hong J, Ha J, Kang J, Kim SY. 2000. ABFs, a family of ABA-responsive element binding factors. J Biol Chem, 275: 1723.

Clarke JD, Liu Y, Klessig DF, Dong X. 1998. Uncoupling PR gene expression from NPR1 and bacterial resistance: characterization of the dominant Arabidopsis cpr6-1 mutant. Plant Cell, 10: 557.

Davies PJ. 2004. Plant hormones: biosythesis, signal transduction, action! London: Kluwer Academic Publishers, 3 rd edition.

Davies RT, Goetz DH, Lasswell J, Anderson MN, Bartel B. 1999. IAR3 encodes an auxin conjugate hydrolase from Arabidopsis. Plant Cell, 11: 365.

De Pater S, Katagiri F, Kijne J, Chua NH. 1994. bZIP proteins bind to a palindromic sequence without an ACGT core located in a seed-specific element of the pea lectin promoter. Plant J, 6: 133.

De Smet I, Voss U, Lau S, Wilson M, Shao N, Timme RE, Swarup R, Kerr I, Hodgman C, Bock R, Bennett M, Jurgens G, Beeckman T. 2011. Unraveling the evolution of auxin signaling. Plant Physiol, 155: 209.

Dharmasiri N, Dharmasiri S, Estelle M. 2005. The F-box protein TIR1 is an auxin receptor. Nature, 435: 441.

Dietrich K, Weltmeier F, Ehlert A, Weiste C, Stahl M, Harter K, Droge-Laser W. 2011. Heterodimers of the Arabidopsis Transcription Factors bZIP1 and bZIP53 Reprogram Amino Acid Metabolism during Low Energy Stress. Plant Cell, 23: 381.

Dreher KA, Brown J, Saw RE, Callis J. 2006. The Arabidopsis Aux/IAA protein family has diversified in degradation and auxin responsiveness. Plant Cell, 18: 699.

Dubrovsky JG, Sauer M, Napsucialy-Mendivil S, Ivanchenko MG, Friml J, Shishkova S, Celenza J, Benkova E. 2008. Auxin acts as a local morphogenetic trigger to specify lateral root founder cells. Proc Natl Acad Sci U S A, 105: 8790.

Earley KW, Shook MS, Brower-Toland B, Hicks L, Pikaard CS. 2007. In vitro specificities of Arabidopsis co-activator histone acetyltransferases: implications for histone hyperacetylation in gene activation. Plant J, 52: 615.

Ellis CM, Nagpal P, Young JC, Hagen G, Guilfoyle TJ, Reed JW. 2005. AUXIN RESPONSE FACTOR1 and AUXIN RESPONSE FACTOR2 regulate senescence and floral organ abscission in Arabidopsis thaliana. Development, 132: 4563.

Epstein E CJ, Slovin J 2002. The biosynthetic pathway for indole-3-acetic acid changes during tomato fruit development. . Plant Growth Regulation, 38: 15.

Foster R, Izawa T, Chua NH. 1994. Plant bZIP proteins gather at ACGT elements. FASEB J, 8: 192.

Friml J. 2010. Subcellular trafficking of PIN auxin efflux carriers in auxin transport. Eur J Cell Biol, 89: 231.

Friml J, Benkova E, Blilou I, Wisniewska J, Hamann T, Ljung K, Woody S, Sandberg G, Scheres B, Jurgens G, Palme K. 2002. AtPIN4 mediates sink-driven auxin gradients and root patterning in Arabidopsis. Cell, 108: 661.

Friml J, Vieten A, Sauer M, Weijers D, Schwarz H, Hamann T, Offringa R, Jurgens G. 2003. Efflux-dependent auxin gradients establish the apical-basal axis of Arabidopsis. Nature, 426: 147.

Fukazawa J, Sakai T, Ishida S, Yamaguchi I, Kamiya Y, Takahashi Y. 2000. Repression of shoot growth, a bZIP transcriptional activator, regulates cell elongation by controlling the level of gibberellins. Plant Cell, 12: 901.

Geisler M, Murphy AS. 2006. The ABC of auxin transport: the role of p-glycoproteins in plant development. FEBS Lett, 580: 1094. 
Glickmann E, Gardan L, Jacquet S, Hussain S, Elasri M, Petit A, Dessaux Y. 1998. Auxin production is a common feature of most pathovars of Pseudomonas syringae. Mol Plant Microbe Interact, 11: 156.

Gray WM, Kepinski S, Rouse D, Leyser O, Estelle M. 2001. Auxin regulates SCF(TIR1)-dependent degradation of AUX/IAA proteins. Nature, 414: 271.

Grossmann K. 2007. Auxin herbicide action: lifting the veil step by step. Plant Signal Behav, 2: 421.

Grunewald W, Friml J. 2010. The march of the PINs: developmental plasticity by dynamic polar targeting in plant cells. EMBO J, 29: 2700.

Guilfoyle TJ, Hagen G. 2007. Auxin response factors. Curr Opin Plant Biol, 10: 453.

Gutierrez RA, Stokes TL, Thum K, Xu X, Obertello M, Katari MS, Tanurdzic M, Dean A, Nero DC, McClung CR, Coruzzi GM. 2008. Systems approach identifies an organic nitrogen-responsive gene network that is regulated by the master clock control gene CCA1. Proc Natl Acad Sci U S A, 105: 4939.

Haagen-Smit AJ DW, Wittwer SH,Murneek AE. 1946. Isolation of 3-indoleacetic acid from immature corn kernels. Am J Bot, 33: 118.

Hagen G, Guilfoyle T. 2002. Auxin-responsive gene expression: genes, promoters and regulatory factors. Plant Mol Biol, 49: 373.

Hanson J, Hanssen M, Wiese A, Hendriks MM, Smeekens S. 2008. The sucrose regulated transcription factor bZIP11 affects amino acid metabolism by regulating the expression of ASPARAGINE SYNTHETASE1 and PROLINE DEHYDROGENASE2. Plant J, 53: 935.

Hardtke CS, Ckurshumova W, Vidaurre DP, Singh SA, Stamatiou G, Tiwari SB, Hagen G, Guilfoyle TJ, Berleth T. 2004. Overlapping and non-redundant functions of the Arabidopsis auxin response factors MONOPTEROS and NONPHOTOTROPIC HYPOCOTYL 4. Development, 131: 1089.

Heil M. 2002. Ecological costs of induced resistance. Curr Opin Plant Biol, 5: 345.

Heinekamp T, Kuhlmann M, Lenk A, Strathmann A, Droge-Laser W. 2002. The tobacco bZIP transcription factor BZI-1 binds to G-box elements in the promoters of phenylpropanoid pathway genes in vitro, but it is not involved in their regulation in vivo. Mol Genet Genomics, 267: 16.

Heinekamp T, Strathmann A, Kuhlmann M, Froissard M, Muller A, Perrot-Rechenmann C, Droge-Laser W. 2004. The tobacco bZIP transcription factor BZI-1 binds the GH3 promoter in vivo and modulates auxin-induced transcription. Plant J, 38: 298.

Heisler MG, Ohno C, Das P, Sieber P, Reddy GV, Long JA, Meyerowitz EM. 2005. Patterns of auxin transport and gene expression during primordium development revealed by live imaging of the Arabidopsis inflorescence meristem. Curr Biol, 15: 1899.

Hoson T, Masuda Y, Sone Y, Misaki A. 1991. Xyloglucan antibodies inhibit auxin-induced elongation and cell wall loosening of azuki bean epicotyls but not of oat coleoptiles. Plant Physiol, 96: 551.

Hummel M, Rahmani F, Smeekens S, Hanson J. 2009. Sucrose-mediated translational control. Ann Bot, 104: 1 .

Hurst HC. 1995. Transcription factors 1: bZIP proteins. Protein Profile, 2: 101.

Ikeda Y, Men S, Fischer U, Stepanova AN, Alonso JM, Ljung K, Grebe M. 2009. Local auxin biosynthesis modulates gradient-directed planar polarity in Arabidopsis. Nat Cell Biol, 11: 731.

Ishida T, Kurata T, Okada K, Wada T. 2008. A genetic regulatory network in the development of trichomes and root hairs. Annu Rev Plant Biol, 59: 365.

Iven T, Strathmann A, Bottner S, Zwafink T, Heinekamp T, Guivarc'h A, Roitsch T, Droge-Laser W. 2010. Homo- and heterodimers of tobacco bZIP proteins counteract as positive or negative regulators of transcription during pollen development. Plant J, 63: 155.

Izawa T, Foster R, Chua NH. 1993. Plant bZIP protein DNA binding specificity. J Mol Biol, 230: 1131.

Jakoby M, Weisshaar B, Droge-Laser W, Vicente-Carbajosa J, Tiedemann J, Kroj T, Parcy F. 2002. bZIP transcription factors in Arabidopsis. Trends Plant Sci, 7: 106.

Jones AM, Im KH, Savka MA, Wu MJ, DeWitt NG, Shillito R, Binns AN. 1998. Auxin-dependent cell expansion mediated by overexpressed auxin-binding protein 1. Science, 282: 1114. 
Kepinski S, Leyser O. 2002. Ubiquitination and auxin signaling: a degrading story. Plant Cell, 14 Suppl: S81.

Kepinski S, Leyser O. 2005. The Arabidopsis F-box protein TIR1 is an auxin receptor. Nature, 435: 446.

Kim J, Harter K, Theologis A. 1997. Protein-protein interactions among the Aux/IAA proteins. Proc Natl Acad Sci U S A, 94: 11786.

Koornneef A, Rindermann K, Gatz C, Pieterse CM. 2008. Histone modifications do not play a major role in salicylate-mediated suppression of jasmonate-induced PDF1.2 gene expression. Commun Integr Biol, 1: 143.

Kornet N, Scheres B. 2009. Members of the GCN5 histone acetyltransferase complex regulate PLETHORA-mediated root stem cell niche maintenance and transit amplifying cell proliferation in Arabidopsis. Plant Cell, 21: 1070.

Kouzarides T. 2007. Chromatin modifications and their function. Cell, 128: 693.

Kovtun Y, Chiu WL, Zeng W, Sheen J. 1998. Suppression of auxin signal transduction by a MAPK cascade in higher plants. Nature, 395: 716.

Lam HM, Peng SS, Coruzzi GM. 1994. Metabolic regulation of the gene encoding glutamine-dependent asparagine synthetase in Arabidopsis thaliana. Plant Physiol, 106: 1347.

Landschulz WH, Johnson PF, McKnight SL. 1988. The leucine zipper: a hypothetical structure common to a new class of DNA binding proteins. Science, 240: 1759.

Lara P, Onate-Sanchez L, Abraham Z, Ferrandiz C, Diaz I, Carbonero P, Vicente-Carbajosa J. 2003. Synergistic activation of seed storage protein gene expression in Arabidopsis by ABI3 and two bZIPs related to OPAQUE2. J Biol Chem, 278: 21003.

Latrasse D, Benhamed M, Henry Y, Domenichini S, Kim W, Zhou DX, Delarue M. 2008. The MYST histone acetyltransferases are essential for gametophyte development in Arabidopsis. BMC Plant Biol, 8: 121.

Leblanc N, David K, Grosclaude J, Pradier JM, Barbier-Brygoo H, Labiau S, Perrot-Rechenmann C. 1999. A novel immunological approach establishes that the auxin-binding protein, Nt-abp1, is an element involved in auxin signaling at the plasma membrane. J Biol Chem, 274: 28314.

Lee JS, Wang S, Sritubtim S, Chen JG, Ellis BE. 2009. Arabidopsis mitogen-activated protein kinase MPK12 interacts with the MAPK phosphatase IBR5 and regulates auxin signaling. Plant J, 57: 975.

Leyser HM, Lincoln CA, Timpte C, Lammer D, Turner J, Estelle M. 1993. Arabidopsis auxinresistance gene AXR1 encodes a protein related to ubiquitin-activating enzyme E1. Nature, 364: 161.

Li X, Clarke JD, Zhang Y, Dong X. 2001. Activation of an EDS1-mediated R-gene pathway in the snc1 mutant leads to constitutive, NPR1-independent pathogen resistance. Mol Plant Microbe Interact, 14: 1131.

Liscum E, Reed JW. 2002. Genetics of Aux/IAA and ARF action in plant growth and development. Plant Mol Biol, 49: 387.

Liu Z, Karmarkar V. 2008. Groucho/Tup1 family co-repressors in plant development. Trends Plant Sci, 13: 137.

Liu ZB, Hagen G, Guilfoyle TJ. 1997. A G-Box-Binding Protein from Soybean Binds to the E1 AuxinResponse Element in the Soybean GH3 Promoter and Contains a Proline-Rich Repression Domain. Plant Physiol, 115: 397.

Liu ZB, Ulmasov T, Shi X, Hagen G, Guilfoyle TJ. 1994. Soybean GH3 promoter contains multiple auxin-inducible elements. Plant Cell, 6: 645.

Ljung K, Bhalerao RP, Sandberg G. 2001. Sites and homeostatic control of auxin biosynthesis in Arabidopsis during vegetative growth. Plant J, 28: 465.

Ljung K, Hull AK, Celenza J, Yamada M, Estelle M, Normanly J, Sandberg G. 2005. Sites and regulation of auxin biosynthesis in Arabidopsis roots. Plant Cell, 17: 1090.

Locatelli F, Bracale M, Magaraggia F, Faoro F, Manzocchi LA, Coraggio I. 2000. The product of the rice myb7 unspliced mRNA dimerizes with the maize leucine zipper Opaque2 and stimulates its activity in a transient expression assay. J Biol Chem, 275: 17619. 
Locatelli S, Piatti P, Motto M, Rossi V. 2009. Chromatin and DNA modifications in the Opaque2mediated regulation of gene transcription during maize endosperm development. Plant Cell, 21: 1410.

Long JA, Ohno C, Smith ZR, Meyerowitz EM. 2006. TOPLESS regulates apical embryonic fate in Arabidopsis. Science, 312: 1520.

Ludwig-Muller J. 2011. Auxin conjugates: their role for plant development and in the evolution of land plants. J Exp Bot, 62: 1757.

Mao Y, Pavangadkar KA, Thomashow MF, Triezenberg SJ. 2006. Physical and functional interactions of Arabidopsis ADA2 transcriptional coactivator proteins with the acetyltransferase GCN5 and with the cold-induced transcription factor CBF1. Biochim Biophys Acta, 1759: 69.

Marchant A, Bhalerao R, Casimiro I, Eklof J, Casero PJ, Bennett M, Sandberg G. 2002. AUX1 promotes lateral root formation by facilitating indole-3-acetic acid distribution between sink and source tissues in the Arabidopsis seedling. Plant Cell, 14: 589.

Mattsson J, Ckurshumova W, Berleth T. 2003. Auxin signaling in Arabidopsis leaf vascular development. Plant Physiol, 131: 1327.

Metallo SJ, Schepartz A. 1997. Certain bZIP peptides bind DNA sequentially as monomers and dimerize on the DNA. Nat Struct Biol, 4: 115.

Michael TP, Mockler TC, Breton G, McEntee C, Byer A, Trout JD, Hazen SP, Shen R, Priest HD, Sullivan CM, Givan SA, Yanovsky M, Hong F, Kay SA, Chory J. 2008. Network discovery pipeline elucidates conserved time-of-day-specific cis-regulatory modules. PLoS Genet, 4: e14.

Millar CB, Grunstein M. 2006. Genome-wide patterns of histone modifications in yeast. Nat Rev Mol Cell Biol, 7: 657.

Mockaitis K, Howell SH. 2000. Auxin induces mitogenic activated protein kinase (MAPK) activation in roots of Arabidopsis seedlings. Plant J, 24: 785.

Moller B, Weijers D. 2009. Auxin control of embryo patterning. Cold Spring Harb Perspect Biol, 1: a001545.

Moubayidin L, Perilli S, Dello Ioio R, Di Mambro R, Costantino P, Sabatini S. 2010. The rate of cell differentiation controls the Arabidopsis root meristem growth phase. Curr Biol, 20: 1138.

Muday GK. 2001. Auxins and tropisms. J Plant Growth Regul, 20: 226.

Muto H, Watahiki MK, Nakamoto D, Kinjo M, Yamamoto KT. 2007. Specificity and similarity of functions of the Aux/IAA genes in auxin signaling of Arabidopsis revealed by promoter-exchange experiments among MSG2/IAA19, AXR2/IAA7, and SLR/IAA14. Plant Physiol, 144: 187.

Nagpal P, Walker LM, Young JC, Sonawala A, Timpte C, Estelle M, Reed JW. 2000. AXR2 encodes a member of the Aux/IAA protein family. Plant Physiol, 123: 563.

Nakamura A, Higuchi K, Goda H, Fujiwara MT, Sawa S, Koshiba T, Shimada Y, Yoshida S. 2003. Brassinolide induces IAA5, IAA19, and DR5, a synthetic auxin response element in Arabidopsis, implying a cross talk point of brassinosteroid and auxin signaling. Plant Physiol, 133: 1843.

Nakamura A, Umemura I, Gomi K, Hasegawa Y, Kitano H, Sazuka T, Matsuoka M. 2006. Production and characterization of auxin-insensitive rice by overexpression of a mutagenized rice IAA protein. Plant J, 46: 297.

Nakazawa M, Yabe N, Ichikawa T, Yamamoto YY, Yoshizumi T, Hasunuma K, Matsui M. 2001. DFL1, an auxin-responsive GH3 gene homologue, negatively regulates shoot cell elongation and lateral root formation, and positively regulates the light response of hypocotyl length. Plant J, $\mathbf{2 5}$ : 213.

Navarro L, Dunoyer P, Jay F, Arnold B, Dharmasiri N, Estelle M, Voinnet O, Jones JD. 2006. A plant miRNA contributes to antibacterial resistance by repressing auxin signaling. Science, 312: 436.

Nole-Wilson S, Azhakanandam S, Franks RG. 2010. Polar auxin transport together with aintegumenta and revoluta coordinate early Arabidopsis gynoecium development. Dev Biol, 346: 181.

O'Shea EK, Rutkowski R, Stafford WF, 3rd, Kim PS. 1989. Preferential heterodimer formation by isolated leucine zippers from fos and jun. Science, 245: 646. 
Okushima Y, Overvoorde PJ, Arima K, Alonso JM, Chan A, Chang C, Ecker JR, Hughes B, Lui A, Nguyen D, Onodera C, Quach H, Smith A, Yu G, Theologis A. 2005. Functional genomic analysis of the AUXIN RESPONSE FACTOR gene family members in Arabidopsis thaliana: unique and overlapping functions of ARF7 and ARF19. Plant Cell, 17: 444.

Ostin A, Kowalyczk M, Bhalerao RP, Sandberg G. 1998. Metabolism of indole-3-acetic acid in Arabidopsis. Plant Physiol, 118: 285.

Ouellet F, Overvoorde PJ, Theologis A. 2001. IAA17/AXR3: biochemical insight into an auxin mutant phenotype. Plant Cell, 13: 829.

Ouyang J, Shao X, Li J. 2000. Indole-3-glycerol phosphate, a branchpoint of indole-3-acetic acid biosynthesis from the tryptophan biosynthetic pathway in Arabidopsis thaliana. Plant J, 24: 327.

Overvoorde P, Fukaki H, Beeckman T. 2010. Auxin control of root development. Cold Spring Harb Perspect Biol, 2: a001537.

Overvoorde PJ, Okushima Y, Alonso JM, Chan A, Chang C, Ecker JR, Hughes B, Liu A, Onodera C, Quach H, Smith A, Yu G, Theologis A. 2005. Functional genomic analysis of the AUXIN/INDOLE-3-ACETIC ACID gene family members in Arabidopsis thaliana. Plant Cell, 17: 3282.

Padmanabhan MS, Kramer SR, Wang X, Culver JN. 2008. Tobacco mosaic virus replicaseauxin/indole acetic acid protein interactions: reprogramming the auxin response pathway to enhance virus infection. $J$ Virol, 82: 2477.

Padmanabhan MS, Shiferaw H, Culver JN. 2006. The Tobacco mosaic virus replicase protein disrupts the localization and function of interacting Aux/IAA proteins. Mol Plant Microbe Interact, 19: 864.

Pandey R, Muller A, Napoli CA, Selinger DA, Pikaard CS, Richards EJ, Bender J, Mount DW, Jorgensen RA. 2002. Analysis of histone acetyltransferase and histone deacetylase families of Arabidopsis thaliana suggests functional diversification of chromatin modification among multicellular eukaryotes. Nucleic Acids Res, 30: 5036.

Pape S, Thurow C, Gatz C. 2010a. The Arabidopsis PR-1 promoter contains multiple integration sites for the coactivator NPR1 and the repressor SNI1. Plant Physiol, 154: 1805.

Pape S, Thurow C, Gatz C. 2010b. Exchanging the as-1-like element of the PR-1 promoter by the as-1 element of the CaMV 35S promoter abolishes salicylic acid responsiveness and regulation by NPR1 and SNI1. Plant Signal Behav, 5.

Park CM. 2007. Auxin homeostasis in plant stress adaptation response. Plant Signal Behav, 2: 306.

Park JE, Park JY, Kim YS, Staswick PE, Jeon J, Yun J, Kim SY, Kim J, Lee YH, Park CM. 2007. GH3-mediated auxin homeostasis links growth regulation with stress adaptation response in Arabidopsis. J Biol Chem, 282: 10036.

Parry G, Calderon-Villalobos LI, Prigge M, Peret B, Dharmasiri S, Itoh H, Lechner E, Gray WM, Bennett M, Estelle M. 2009. Complex regulation of the TIR1/AFB family of auxin receptors. Proc Natl Acad Sci U S A, 106: 22540.

Peret B, Larrieu A, Bennett MJ. 2009. Lateral root emergence: a difficult birth. J Exp Bot, 60: 3637.

Petersson SV, Johansson AI, Kowalczyk M, Makoveychuk A, Wang JY, Moritz T, Grebe M, Benfey PN, Sandberg G, Ljung K. 2009. An auxin gradient and maximum in the Arabidopsis root apex shown by high-resolution cell-specific analysis of IAA distribution and synthesis. Plant Cell, 21: 1659.

Petrasek J, Mravec J, Bouchard R, Blakeslee JJ, Abas M, Seifertova D, Wisniewska J, Tadele Z, Kubes M, Covanova M, Dhonukshe P, Skupa P, Benkova E, Perry L, Krecek P, Lee OR, Fink GR, Geisler M, Murphy AS, Luschnig C, Zazimalova E, Friml J. 2006. PIN proteins perform a rate-limiting function in cellular auxin efflux. Science, 312: 914.

Pieterse CM, Leon-Reyes A, Van der Ent S, Van Wees SC. 2009. Networking by small-molecule hormones in plant immunity. Nat Chem Biol, 5: 308.

Pitts RJ, Cernac A, Estelle M. 1998. Auxin and ethylene promote root hair elongation in Arabidopsis. Plant J, 16: 553.

Pozo JC, Timpte C, Tan S, Callis J, Estelle M. 1998. The ubiquitin-related protein RUB1 and auxin response in Arabidopsis. Science, 280: 1760. 
Pysh LD, Aukerman MJ, Schmidt RJ. 1993. OHP1: a maize basic domain/leucine zipper protein that interacts with opaque2. Plant Cell, 5: 227.

Radchuk R, Radchuk V, Weschke W, Borisjuk L, Weber H. 2006. Repressing the expression of the SUCROSE NONFERMENTING-1-RELATED PROTEIN KINASE gene in pea embryo causes pleiotropic defects of maturation similar to an abscisic acid-insensitive phenotype. Plant Physiol, 140: 263.

Rahman A, Hosokawa S, Oono Y, Amakawa T, Goto N, Tsurumi S. 2002. Auxin and ethylene response interactions during Arabidopsis root hair development dissected by auxin influx modulators. Plant Physiol, 130: 1908.

Rahmani F, Hummel M, Schuurmans J, Wiese-Klinkenberg A, Smeekens S, Hanson J. 2009. Sucrose control of translation mediated by an upstream open reading frame-encoded peptide. Plant Physiol, 150: 1356.

Rampey RA, LeClere S, Kowalczyk M, Ljung K, Sandberg G, Bartel B. 2004. A family of auxinconjugate hydrolases that contributes to free indole-3-acetic acid levels during Arabidopsis germination. Plant Physiol, 135: 978.

Raven JA. 1975. Transport of indoleacetic acid in plant cells in relation to $\mathrm{pH}$ and electrical potential gradients, and its significance for polar IAA transport. New Phytol, 74: 163.

Reed JW. 2001. Roles and activities of Aux/IAA proteins in Arabidopsis. Trends Plant Sci, 6: 420.

Remington DL, Vision TJ, Guilfoyle TJ, Reed JW. 2004. Contrasting modes of diversification in the Aux/IAA and ARF gene families. Plant Physiol, 135: 1738.

Rensing SA, Lang D, Zimmer AD, Terry A, Salamov A, Shapiro H, Nishiyama T, Perroud PF, Lindquist EA, Kamisugi Y, Tanahashi T, Sakakibara K, Fujita T, Oishi K, Shin IT, Kuroki Y, Toyoda A, Suzuki Y, Hashimoto S, Yamaguchi K, Sugano S, Kohara Y, Fujiyama A, Anterola A, Aoki S, Ashton N, Barbazuk WB, Barker E, Bennetzen JL, Blankenship R, Cho SH, Dutcher SK, Estelle M, Fawcett JA, Gundlach H, Hanada K, Heyl A, Hicks KA, Hughes J, Lohr M, Mayer K, Melkozernov A, Murata T, Nelson DR, Pils B, Prigge M, Reiss B, Renner T, Rombauts S, Rushton PJ, Sanderfoot A, Schween G, Shiu SH, Stueber K, Theodoulou FL, Tu H, Van de Peer Y, Verrier PJ, Waters E, Wood A, Yang L, Cove D, Cuming AC, Hasebe M, Lucas S, Mishler BD, Reski R, Grigoriev IV, Quatrano RS, Boore JL. 2008. The Physcomitrella genome reveals evolutionary insights into the conquest of land by plants. Science, 319: 64.

Robert HS, Friml J. 2009. Auxin and other signals on the move in plants. Nat Chem Biol, 5: 325.

Rogg LE, Bartel B. 2001. Auxin signaling: derepression through regulated proteolysis. Dev Cell, 1: 595.

Rook F, Gerrits N, Kortstee A, van Kampen M, Borrias M, Weisbeek P, Smeekens S. 1998. Sucrosespecific signalling represses translation of the Arabidopsis ATB2 bZIP transcription factor gene. Plant J, 15: 253.

Ruegger M, Dewey E, Gray WM, Hobbie L, Turner J, Estelle M. 1998. The TIR1 protein of Arabidopsis functions in auxin response and is related to human SKP2 and yeast grr1p. Genes Dev, 12: 198.

Rugner A, Frohnmeyer H, Nake C, Wellmer F, Kircher S, Schafer E, Harter K. 2001. Isolation and characterization of four novel parsley proteins that interact with the transcriptional regulators CPRF1 and CPRF2. Mol Genet Genomics, 265: 964.

Rymen B, Fiorani F, Kartal F, Vandepoele K, Inze D, Beemster GT. 2007. Cold nights impair leaf growth and cell cycle progression in maize through transcriptional changes of cell cycle genes. Plant Physiol, 143: 1429.

Sabatini S, Beis D, Wolkenfelt H, Murfett J, Guilfoyle T, Malamy J, Benfey P, Leyser O, Bechtold N, Weisbeek P, Scheres B. 1999. An auxin-dependent distal organizer of pattern and polarity in the Arabidopsis root. Cell, 99: 463.

Santner A, Calderon-Villalobos LI, Estelle M. 2009. Plant hormones are versatile chemical regulators of plant growth. Nat Chem Biol, 5: 301.

Satoh R, Fujita Y, Nakashima K, Shinozaki K, Yamaguchi-Shinozaki K. 2004. A novel subgroup of bZIP proteins functions as transcriptional activators in hypoosmolarity-responsive expression of the ProDH gene in Arabidopsis. Plant Cell Physiol, 45: 309. 
Savic B, Tomic S, Magnus V, Gruden K, Barle K, Grenkovic R, Ludwig-Muller J, Salopek-Sondi B. 2009. Auxin amidohydrolases from Brassica rapa cleave the alanine conjugate of indolepropionic acid as a preferable substrate: a biochemical and modeling approach. Plant Cell Physiol, 50: 1587.

Scacchi E, Salinas P, Gujas B, Santuari L, Krogan N, Ragni L, Berleth T, Hardtke CS. 2010. Spatiotemporal sequence of cross-regulatory events in root meristem growth. Proc Natl Acad Sci U S A, 107: 22734.

Scarpella E, Marcos D, Friml J, Berleth T. 2006. Control of leaf vascular patterning by polar auxin transport. Genes Dev, 20: 1015.

Schindler U, Beckmann H, Cashmore AR. 1992. TGA1 and G-box binding factors: two distinct classes of Arabidopsis leucine zipper proteins compete for the G-box-like element TGACGTGG. Plant Cell, 4: 1309.

Senn AP, Goldsmith MH. 1988. Regulation of electrogenic proton pumping by auxin and fusicoccin as related to the growth of Avena coleoptiles. Plant Physiol, 88: 131.

Servet C, Conde e Silva N, Zhou DX. 2010. Histone acetyltransferase AtGCN5/HAG1 is a versatile regulator of developmental and inducible gene expression in Arabidopsis. Mol Plant, 3: 670.

Sheldrake Ra. 1974. Carrier-mediated auxin transport. Planta, 118: 101.

Shin R, Burch AY, Huppert KA, Tiwari SB, Murphy AS, Guilfoyle TJ, Schachtman DP. 2007. The Arabidopsis transcription factor MYB77 modulates auxin signal transduction. Plant Cell, 19: 2440.

Shishova M, Lindberg S. 2004. Auxin induces an increase of $\mathrm{Ca} 2+$ concentration in the cytosol of wheat leaf protoplasts. J Plant Physiol, 161: 937.

Shoji K, Addicott FT, Swets WA. 1951. Auxin in Relation to Leaf Blade Abscission. Plant Physiol, 26: 189.

Siberil Y, Doireau P, Gantet P. 2001. Plant bZIP G-box binding factors. Modular structure and activation mechanisms. Eur J Biochem, 268: 5655.

Smeekens S, Ma J, Hanson J, Rolland F. 2010. Sugar signals and molecular networks controlling plant growth. Curr Opin Plant Biol, 13: 274.

Snow. 1935. Activation of cambial growth by pure hormones. New Phytol, 34: 347.

Sorefan K, Girin T, Liljegren SJ, Ljung K, Robles P, Galvan-Ampudia CS, Offringa R, Friml J, Yanofsky MF, Ostergaard L. 2009. A regulated auxin minimum is required for seed dispersal in Arabidopsis. Nature, 459: 583.

Spoel SH, Dong X. 2008. Making sense of hormone crosstalk during plant immune responses. Cell Host Microbe, 3: 348.

Staswick PE. 2009. The tryptophan conjugates of jasmonic and indole-3-acetic acids are endogenous auxin inhibitors. Plant Physiol, 150: 1310.

Staswick PE, Serban B, Rowe M, Tiryaki I, Maldonado MT, Maldonado MC, Suza W. 2005. Characterization of an Arabidopsis enzyme family that conjugates amino acids to indole-3-acetic acid. Plant Cell, 17: 616.

Stepanova AN, Robertson-Hoyt J, Yun J, Benavente LM, Xie DY, Dolezal K, Schlereth A, Jurgens G, Alonso JM. 2008. TAA1-mediated auxin biosynthesis is essential for hormone crosstalk and plant development. Cell, 133: 177.

Stockinger EJ, Mao Y, Regier MK, Triezenberg SJ, Thomashow MF. 2001. Transcriptional adaptor and histone acetyltransferase proteins in Arabidopsis and their interactions with CBF1, a transcriptional activator involved in cold-regulated gene expression. Nucleic Acids Res, 29: 1524.

Strathmann A, Kuhlmann M, Heinekamp T, Droge-Laser W. 2001. BZI-1 specifically heterodimerises with the tobacco bZIP transcription factors BZI-2, BZI-3/TBZF and BZI-4, and is functionally involved in flower development. Plant J, 28: 397.

Sulpice R, Pyl ET, Ishihara H, Trenkamp S, Steinfath M, Witucka-Wall H, Gibon Y, Usadel B, Poree F, Piques MC, Von Korff M, Steinhauser MC, Keurentjes JJ, Guenther M, Hoehne M, Selbig J, Fernie AR, Altmann T, Stitt M. 2009. Starch as a major integrator in the regulation of plant growth. Proc Natl Acad Sci U S A, 106: 10348. 
Swarup R, Friml J, Marchant A, Ljung K, Sandberg G, Palme K, Bennett M. 2001. Localization of the auxin permease AUX1 suggests two functionally distinct hormone transport pathways operate in the Arabidopsis root apex. Genes Dev, 15: 2648.

Szemenyei H, Hannon M, Long JA. 2008. TOPLESS mediates auxin-dependent transcriptional repression during Arabidopsis embryogenesis. Science, 319: 1384.

Takase T, Nakazawa M, Ishikawa A, Kawashima M, Ichikawa T, Takahashi N, Shimada H, Manabe K, Matsui M. 2004. ydk1-D, an auxin-responsive GH3 mutant that is involved in hypocotyl and root elongation. Plant J, 37: 471.

Tam YY, Epstein E, Normanly J. 2000. Characterization of auxin conjugates in Arabidopsis. Low steady-state levels of indole-3-acetyl-aspartate, indole-3-acetyl-glutamate, and indole-3-acetylglucose. Plant Physiol, 123: 589.

Tan X, Calderon-Villalobos LI, Sharon M, Zheng C, Robinson CV, Estelle M, Zheng N. 2007. Mechanism of auxin perception by the TIR1 ubiquitin ligase. Nature, 446: 640.

Tanaka H, Dhonukshe P, Brewer PB, Friml J. 2006. Spatiotemporal asymmetric auxin distribution: a means to coordinate plant development. Cell Mol Life Sci, 63: 2738.

Tao Y, Ferrer JL, Ljung K, Pojer F, Hong F, Long JA, Li L, Moreno JE, Bowman ME, Ivans LJ, Cheng Y, Lim J, Zhao Y, Ballare CL, Sandberg G, Noel JP, Chory J. 2008. Rapid synthesis of auxin via a new tryptophan-dependent pathway is required for shade avoidance in plants. Cell, 133: 164.

Tatematsu K, Kumagai S, Muto H, Sato A, Watahiki MK, Harper RM, Liscum E, Yamamoto KT. 2004. MASSUGU2 encodes Aux/IAA19, an auxin-regulated protein that functions together with the transcriptional activator NPH4/ARF7 to regulate differential growth responses of hypocotyl and formation of lateral roots in Arabidopsis thaliana. Plant Cell, 16: 379.

Thelander M, Olsson T, Ronne H. 2004. Snf1-related protein kinase 1 is needed for growth in a normal day-night light cycle. EMBO J, 23: 1900.

Theologis A, Huynh TV, Davis RW. 1985. Rapid induction of specific mRNAs by auxin in pea epicotyl tissue. J Mol Biol, 183: 53.

Thimann. 1977. Hormones action in the whole life of plants. University of Massachussetts Press, Amherst.

Thimann KV. 1938. Hormones and the Analysis of Growth. Plant Physiol, 13: 437.

Tian Q, Reed JW. 1999. Control of auxin-regulated root development by the Arabidopsis thaliana SHY2/IAA3 gene. Development, 126: 711.

Tian Q, Uhlir NJ, Reed JW. 2002. Arabidopsis SHY2/IAA3 inhibits auxin-regulated gene expression. Plant Cell, 14: 301.

Tiwari SB, Hagen G, Guilfoyle T. 2003. The roles of auxin response factor domains in auxin-responsive transcription. Plant Cell, 15: 533.

Tiwari SB, Hagen G, Guilfoyle TJ. 2004. Aux/IAA proteins contain a potent transcriptional repression domain. Plant Cell, 16: 533.

Topalidou I, Thireos G. 2003. Gcn4 occupancy of open reading frame regions results in the recruitment of chromatin-modifying complexes but not the mediator complex. EMBO Rep, 4: 872.

Tsurumi S WS. 1980. Transport of shoot- and cotyledon-applied indole-3-acetic acid to Vicia faba root. Plant and Cell Physiology, 21: 803.

Ulmasov T, Hagen G, Guilfoyle TJ. 1997a. ARF1, a transcription factor that binds to auxin response elements. Science, 276: 1865.

Ulmasov T, Hagen G, Guilfoyle TJ. 1999. Dimerization and DNA binding of auxin response factors. Plant J, 19: 309.

Ulmasov T, Liu ZB, Hagen G, Guilfoyle TJ. 1995. Composite structure of auxin response elements. Plant Cell, 7: 1611.

Ulmasov T, Murfett J, Hagen G, Guilfoyle TJ. 1997b. Aux/IAA proteins repress expression of reporter genes containing natural and highly active synthetic auxin response elements. Plant Cell, 9: 1963.

Vanneste S, Friml J. 2009. Auxin: a trigger for change in plant development. Cell, 136: 1005. 
Vernoux T, Besnard F, Traas J. 2010. Auxin at the shoot apical meristem. Cold Spring Harb Perspect Biol, 2: a001487.

Vinson C, Myakishev M, Acharya A, Mir AA, Moll JR, Bonovich M. 2002. Classification of human B-ZIP proteins based on dimerization properties. Mol Cell Biol, 22: 6321.

Vlachonasios KE, Thomashow MF, Triezenberg SJ. 2003. Disruption mutations of ADA2b and GCN5 transcriptional adaptor genes dramatically affect Arabidopsis growth, development, and gene expression. Plant Cell, 15: 626.

Walker JC, Key JL. 1982. Isolation of cloned cDNAs to auxin-responsive poly(A)RNAs of elongating soybean hypocotyl. Proc Natl Acad Sci U S A, 79: 7185.

Wang D, Pajerowska-Mukhtar K, Culler AH, Dong X. 2007. Salicylic acid inhibits pathogen growth in plants through repression of the auxin signaling pathway. Curr Biol, 17: 1784.

Wang YH, Irving HR. 2011. Developing a model of plant hormone interactions. Plant Signal Behav, 6.

Weijers D, Benkova E, Jager KE, Schlereth A, Hamann T, Kientz M, Wilmoth JC, Reed JW, Jurgens G. 2005. Developmental specificity of auxin response by pairs of ARF and Aux/IAA transcriptional regulators. EMBO J, 24: 1874.

Weijers D, Jurgens G. 2004. Funneling auxin action: specificity in signal transduction. Curr Opin Plant Biol, 7: 687.

Weltmeier F, Ehlert A, Mayer CS, Dietrich K, Wang X, Schutze K, Alonso R, Harter K, VicenteCarbajosa J, Droge-Laser W. 2006. Combinatorial control of Arabidopsis proline dehydrogenase transcription by specific heterodimerisation of bZIP transcription factors. EMBO J, 25: 3133.

Weltmeier F, Rahmani F, Ehlert A, Dietrich K, Schutze K, Wang X, Chaban C, Hanson J, Teige M, Harter K, Vicente-Carbajosa J, Smeekens S, Droge-Laser W. 2009. Expression patterns within the Arabidopsis C/S1 bZIP transcription factor network: availability of heterodimerization partners controls gene expression during stress response and development. Plant Mol Biol, 69: 107.

Went F, Thimann KV. 1937. Phytohormones. MacMillan Company, New York.

Whippo CW, Hangarter RP. 2006. Phototropism: bending towards enlightenment. Plant Cell, 18: 1110.

Wiese A, Elzinga N, Wobbes B, Smeekens S. 2004. A conserved upstream open reading frame mediates sucrose-induced repression of translation. Plant Cell, 16: 1717.

Wilson AK, Pickett FB, Turner JC, Estelle M. 1990. A dominant mutation in Arabidopsis confers resistance to auxin, ethylene and abscisic acid. Mol Gen Genet, 222: 377.

Woodward AW, Bartel B. 2005. Auxin: regulation, action, and interaction. Ann Bot, 95: 707.

Worley CK, Zenser N, Ramos J, Rouse D, Leyser O, Theologis A, Callis J. 2000. Degradation of Aux/IAA proteins is essential for normal auxin signalling. Plant J, 21: 553.

Zeiger Ta. 2006. Plant Physiology. 4th edition.

Zhang L, Su X, Liu S, Knapp AR, Parthun MR, Marcucci G, Freitas MA. 2007. Histone H4 Nterminal acetylation in Kasumi-1 cells treated with depsipeptide determined by acetic acid-urea polyacrylamide gel electrophoresis, amino acid coded mass tagging, and mass spectrometry. $J$ Proteome Res, 6: 81.

Zhao Y. 2010. Auxin biosynthesis and its role in plant development. Annu Rev Plant Biol, 61: 49.

Zhao Y, Christensen SK, Fankhauser C, Cashman JR, Cohen JD, Weigel D, Chory J. 2001. A role for flavin monooxygenase-like enzymes in auxin biosynthesis. Science, 291: 306. 


\section{Danksagung}

Diesen Abschnitt meiner Doktorarbeit möchte ich nutzen, um mich bei all denjenigen $\mathrm{zu}$ bedanken, die mich während der letzten vier Jahre auf vielfältige Weise unterstützt haben und damit maßgeblich zum Gelingen der Arbeit beigetragen haben.

Als Erstes möchte ich mich hiermit bei Prof. Dr. Wolfgang Dröge-Laser bedanken, dass er es mir ermöglicht hat meine Dissertation in seiner Arbeitsgruppe anzufertigen. Für die zahllosen Besprechungen und den regen Ideenaustausch bin ich ihm genauso dankbar wie für sein entgegengebrachtes Vertrauen und den Freiraum, den er mir bei der Bearbeitung der Arbeit gelassen hat.

Des Weiteren möchte ich mich bei Professor Dr. Christiane Gatz bedanken. Zum einen für die Übernahme des Korreferats und zum anderen dafür, dass sie es mir ermöglicht hat, die stressige Endphase meiner Arbeit in ihrem Labor zu beenden.

Meinen Laborkollegen Tim(i/ä), Kat(h)rin, Stefan(i), Andrea (die alte), Laura, Nora, Caro, Inken, Jenny, Johanna und Fridtjof, sowie meiner Diplomandin Andrea (die neue) möchte ich ganz herzlich für die schöne Zeit danken. Ohne die Albereien und eure Hilfsbereitschaft wäre diese Arbeit wohl nicht zu Stande gekommen und sicher nicht so lustig über die Bühne gegangen.

Ein weiteres Dankeschön geht auch an alle Mitstreiter aus den Arbeitsgruppen von Frau Prof. Gatz, Herrn Prof. Lipka und Herrn Prof. Schirawski. Vor allem möchte ich mich hier bei Guido, Anna, Ronny, Larissa und Corinna für die vielfältige Hilfe bedanken. Des Weiteren möchte ich auch Prof. Volker Lipka und Prof. Jan Schirawski für die Bereitstellung ihrer Laborgeräte und Rene Fuchs, Christian Löfke und Michael Reusche für die Einführung in die konfokale Mikroskopie danken.

Auch als nicht voll-assoziiertes Mitglied der bZIP Forschergruppe möchte ich mich dennoch bei den Kooperationspartnern Sjef Smeekens und Jesus Vicente-Carbajosa für die abwechselungsreichen und spannenden Aufenthalte in Madrid und Utrecht bedanken. Auch bei Johannes Hanson, sowie bei Micha Hansen möchte ich mich für den Mikroarray Auswertungs-Crashkurs und die Einführung in $\mathrm{R}$ bzw. für die Erkundung Utrechts bedanken. Des Weiteren möchte ich Klaus Harter für seine Hilfe bei der Durchsicht der Manuskripte danken und dass ich in seiner Abteilung einen Einblick in die Bioinformatik bekommen konnte.

Ein extra Dankeschön geht in diesem Zusammenhang an Dr. Kenneth Berendzen. Danke für die viele Arbeit an den Promotor Analysen und an unserem Manuskript und für den schönen Aufenthalt in Tübingen!

Meinen Eltern, Virginia und Hans-Gerd sowie Christines Eltern, Helmut und Luise möchte ich für ihre vielfältige Unterstützung danken.

Mein aller größter Dank gilt meiner Freundin Christine. Bei allen Krisen der Doktorarbeit hat sie mich liebevoll unterstützt und mich so über die letzten vier Jahre gebracht. Ich danke dir!!! 


\section{Curriculum Vitae}

\section{Personal data}

$\begin{array}{ll}\text { name: } & \text { Christoph Weiste } \\ \text { date of birth: } & 23.08 .1980 \\ \text { place of birth: } & \text { Koblenz }\end{array}$

\section{Primary and secondary education}

1987 until 1991

1991 until 2000

Graduation:

07/2000 until 05/2001

\section{Tertiary education}

10/2001 until 11/2006

02/2006 until 10/2006

from $03 / 2007$
Primary school (Martin-Luther-Grundschule), Brilon

Grammar school (Gymnasium Petrinum), Brilon

Abitur / matriculation standard

Civilian service (Arbeiterwohlfahrt), Brilon

Georg-August-Universität Göttingen, Studies of biology, Specialization: molecular biology

Graduation: Diploma in biology

Graduant at the Department "Molekularbiologie und Physiologie der Pflanze" at the Albrecht-von-HallerInstitute, University of Göttingen; Diploma thesis title: „Etablierung einer Methode zur in planta ORFeom Analyse - Generierung von Arabidopsis thaliana Transkriptionsfaktor-Überexpressionslinien und deren Screening auf erhöhte Stresstoleranz".

$\mathrm{PhD}$ student at the Department "Molekularbiologie und Physiologie der Pflanze" at the Albrecht-von-HallerInstitute, University of Göttingen; $\mathrm{PhD}$ thesis title: „Arabidopsis basic leucine Zipper transcription factors function as quantitative modulators of auxin mediated transcription“" 


\section{Publications}

Weiste C., Iven T., Fischer U., Oñate-Sánchez L. and Dröge-Laser W. (2007): In planta ORFeome analysis by large-scale over-expression of GATEWAY-compatible cDNA clones: screening of ERF transcription factors involved in abiotic stress defense. The Plant Journal 52 (2): 382 - 390.

Dietrich K., Weltmeier F., Ehlert A., Weiste C., Stahl M., Harter K., Dröge-Laser W. (2011): Heterodimers of the Arabidopsis Transcription Factors bZIP1 and bZIP53 Reprogram Amino Acid Metabolism during Low Energy Stress. The Plant Cell 23 (1): $381-395$.

Wehner N., Weiste C., Dröge-Laser W. (2011): Molecular Screening Tools to Study Arabidopsis Transcription Factors. Frontiers in Plant Physiology, doi: 10.3389.

\section{Publications (submitted/in preparation)}

Weiste C. and Dröge-Laser W. (submitted): G-box related cis-Elements and their cognate bZIP Transcription Factors function as quantitative Modulators of Auxinmediated growth responses.

Berendzen K. W.*, Weiste C.*, Wanke D., Kilian J., Harter K. and Dröge-Laser W. (submitted): Bioinformatic cis-element analysis performed in Arabidopsis and rice reveals bZIP- and MYB-related binding sites as coupling elements in AuxRE mediated auxin responsive transcription. * joint first-authors

Weiste C. and Dröge-Laser W. (in preparation): Arabidopsis AtbZIP11-related transcription factors modulate auxin-mediated transcription by recruiting the histone acetylation machinery. 